\title{
Self-Sorting, Sergeant-Soldier, and Majority Rules Phenomena in Crystals and Gels of Polyurethane Model Compounds
}

\author{
by
}

\author{
Anita Chan
}

A thesis submitted to the Faculty of Graduate and Postdoctoral Affairs in partial fulfillment of the requirements for the degree of

Doctor of Philosophy

in

Chemistry

Carleton University

Ottawa, Ontario

(C) Copyright

Anita Chan, 2016 


\section{ABSTRACT}

Hydrogen bond-mediated self-assembling biscarbamates have been studied by our group as model compounds of polyurethanes with respect to the crystallization and gelation behaviour in both solid state and solution phase respectively. The aim of my thesis is to investigate the effects on the blending and the gelation of biscarbamates with different alkyl side chain lengths. Melt blending of these molecules shows molecular selectivity and self-sorting behaviour leading to immiscibility. We revealed that the difference in the growth rates of the individual species is responsible for the self-sorting behaviour in these non-chiral synthetic compounds. We discussed methods to bridge the self and non-self that would lead to a more homogeneous system. The gelation properties of biscarbamates with an odd number of carbon atoms in the alkyl side chains, such as the critical gelation concentration, gelation time, gelation temperature, and morphology of the gel fibres were examined. Biscarbamates show odd-even effects in their thermal and gelation behaviours as a function of carbon atom parity in the alkyl side chains in a similar manner to their crystallization behaviours. The blending of the biscarbamate gels shows three different types of blending behaviours. The $\mathrm{C}_{8} / \mathrm{C}_{9}$ blend and any gel blends composed of a biscarbamate smaller than $\mathrm{C}_{6}$ display the sergeant-soldier behaviour. The majority rules principle was observed for odd-odd gels blends with an intermediate difference in side chain length. The odd-odd gels blends with a small difference in side chain length self-sort. Organogels with a series of biscarbamates as gelators were prepared using microwave (MW) heating source as well as conventional heating. Biscarbamates with alkyl side chain lengths varying from $\mathrm{C}_{5}$ to $\mathrm{C}_{18}$ were used, with six solvents having dipole moments ranging from 0.07 to $4.3 \mathrm{D}$. The minimum gelation concentration and the amount of heating for 
dissolution were significantly reduced with MW heating with benzonitrile, compared to the conventional heating for all the side chain lengths of the biscarbamates. MW heating is found to be effective with solvents possessing large dipole moments. Although the gels consist of fibers using both methods, an inherent orientation of these fibers was seen with MW heating versus conventional heating methods. 


\section{ACKNOWLEDGEMENTS}

I am grateful for the opportunity to have pursued my $\mathrm{PhD}$ degree under the supervision of Professor P. R. Sundararajan. He has been a mentor throughout the years, and I am thankful to have benefitted from his wisdom and guidance. I would like to acknowledge the financial support of NSERC (Canada), Xerox Research Centre, and Carleton University. I would also like to acknowledge Dr. Jianqun Wang and the Carleton University Nano Imaging Facility for the generous help and the use of their instruments. I sincerely acknowledge the assistance received from Keith Bourque, Tony O’Neil, Jim Logan, and the Carleton chemistry department. I would like to thank my colleagues Dr. Elianne Dahan, Dr. Kamal Khan, Dr. Molla Rafiq Islam, and Chettichipalayam P. Sakthidharan for help and support. Finally I would like to thank my loving partner Ilya Hekimi for his support and patience over the years. 


\section{TABLE OF CONTENTS}

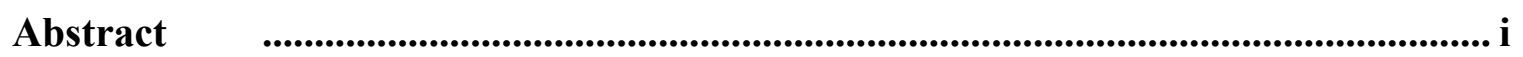

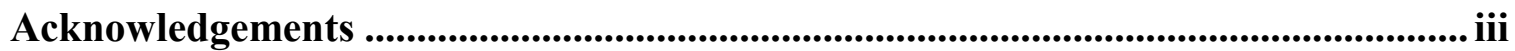

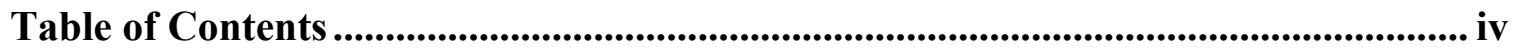

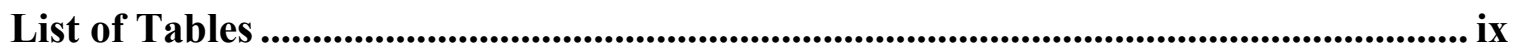

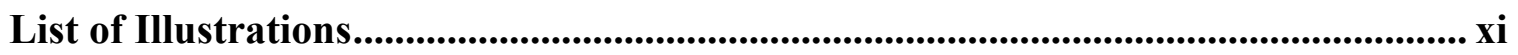

Chapter 1: Introduction ................................................................................................................ 1

1.1 Molecular Self-Assembly ................................................................ 2

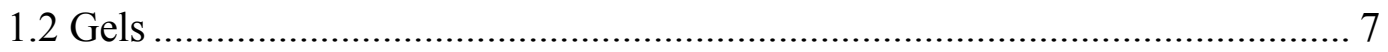

1.2.1 Supramolecular Gels vs. Polymer Gels ........................................ 8

1.2.2 The Principles of Gelation for Small Organic Molecules.................. 11

1.2.3 Properties of Supramolecular Gels ............................................ 15

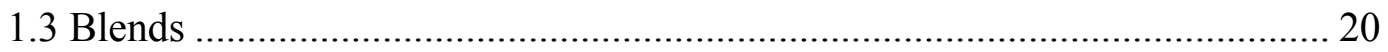

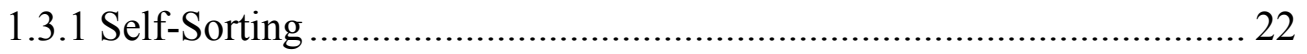

1.3.2 Sergeant-Soldier Effect ............................................................ 23

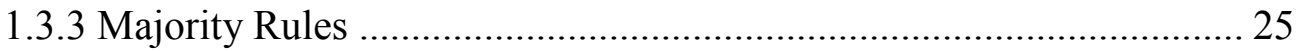

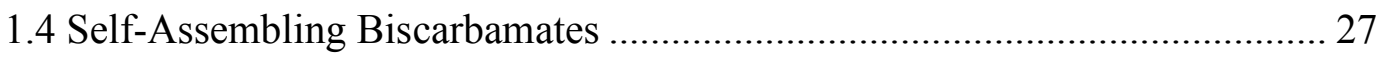

1.4.1 Applications of Carbamates and Biscarbamates............................. 28

1.4.2 Investigation Using Biscarbamates ............................................. 29 
1.5 Thesis Objectives 31

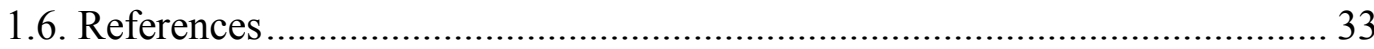

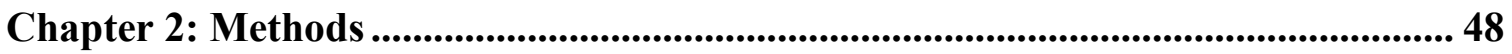

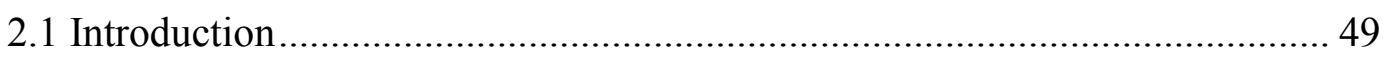

2.2 Biscarbamate Sample Preparations ............................................................ 49

2.2.1 Biscarbamate Synthesis ........................................................ 49

2.2.2 Biscarbamate Blends Preparation ............................................. 52

2.2.3 Biscarbamate Gels Using Conventional Heating.............................. 53

2.2.3.1 Conventional Heating ........................................................ 53

2.2.3.2 Microwave Heating........................................................... 53

2.3 Characterization of the Biscarbamates........................................................ 55

2.3.1 FTIR Spectra of the biscarbamates ............................................. 55

2.3.2 The ${ }^{1} \mathrm{H}$ NMR spectra of the biscarbamates..................................... 59

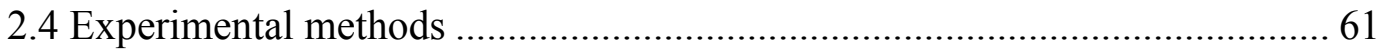

2.4.1 Differential Scanning Calorimetry ............................................. 62

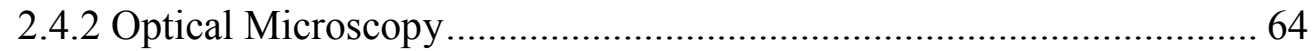

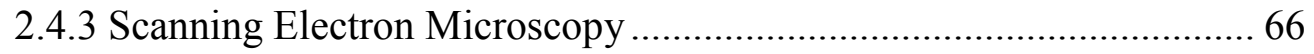

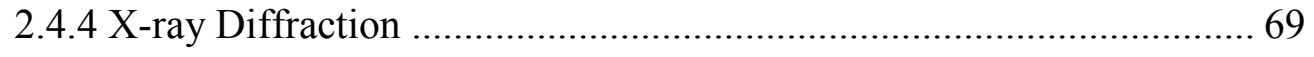

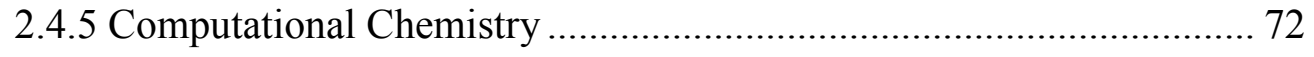


Chapter 3: Inhibiting the Self-Sorting Behaviour in the Blends of a Homologous Set of Biscarbamates ............................................................................................ 79

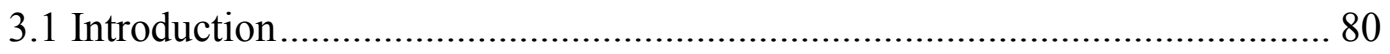

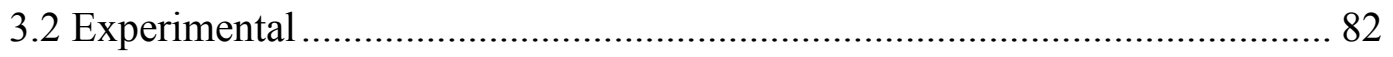

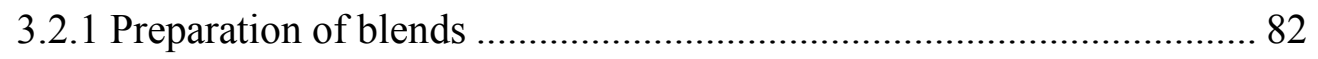

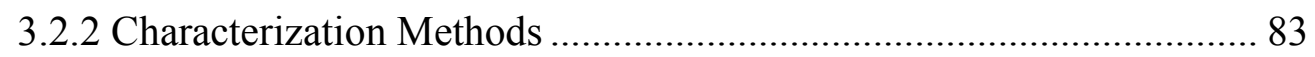

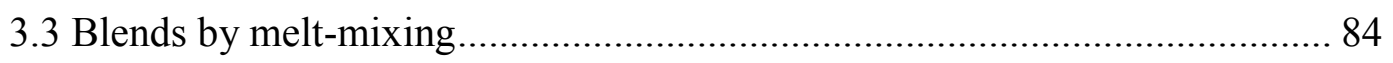

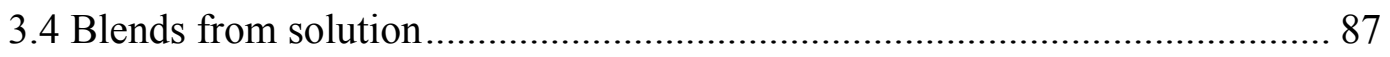

3.5 Solvent Vapor and Thermal Annealing ...................................................... 105

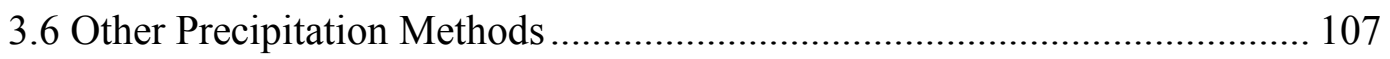

3.7 Odd and Even Biscarbamate Blends.......................................................... 111

3.8 A Third Component as a Compatibilizer ....................................................... 114

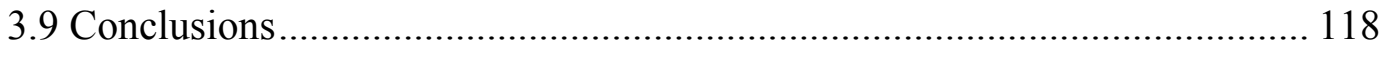

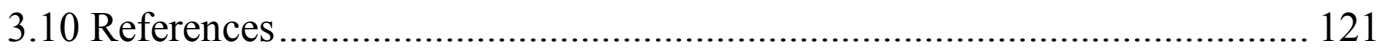

Chapter 4: Influence of the Length of the Alkyl Side Chains on the Gelation of NonChiral Biscarbamates ................................................................................ 125

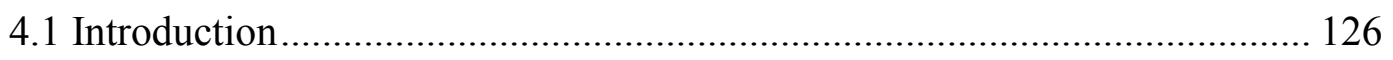

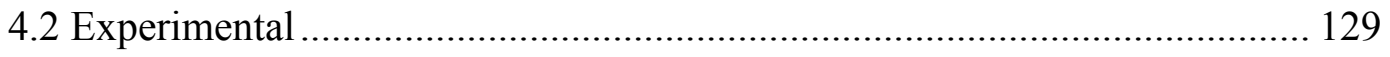

4.2.1 Synthesis of the Biscarbamates......................................................... 129

4.2.2 Preparation of the Gels and Xerogels ................................................ 129 
4.3 Gelation Behaviour of Biscarbamates

4.4 Thermal Behaviour of Biscarbamates

4.5 Morphology of Biscarbamate Gels

4.6 Gel Aging... 144

4.7 Conclusions. 147

4.8 References. 148

Chapter 5: Self-Sorting, Sergeant-Soldier and Majority Rules Effects of Blends of Non-Chiral Biscarbamates Gels 154

5.1 Introduction 155

5.2 Experimental 157

5.2.1 Synthesis of the Biscarbamates 157

5.2.2 Preparation of the Gels and Xerogels 157

5.2.3 Characterization of the Methods 158

5.3 Low Molecular Weight Blends 159

5.4 Special Case of $\mathrm{C}_{8}$ and $\mathrm{C}_{9}$ Biscarbamate Gel Blends 168

5.5 Biscarbamate Gels Blends with a Difference of Four $\mathrm{CH}_{2}$ Groups in the SideChain Length. 173

5.6 Gel Blends of Biscarbamates with a Difference of Two $\mathrm{CH}_{2}$ Groups in the SideChain Length 179

5.7 Conclusions 182 
Chapter 6: Biscarbamate Organogelation Using Microwave Heating Source........ 188

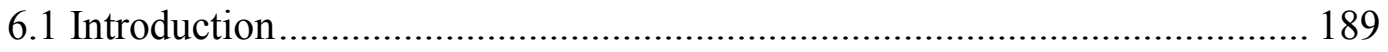

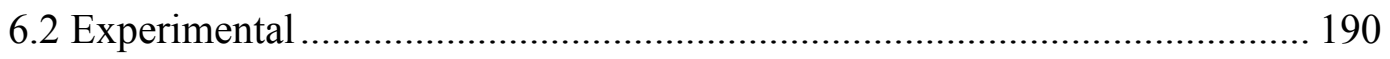

6.2.1 Preparation of the Gels and Xerogels ............................................... 190

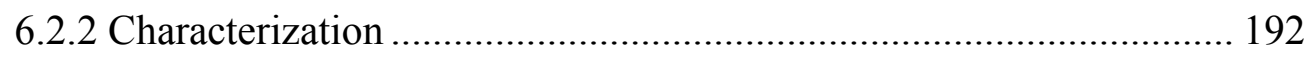

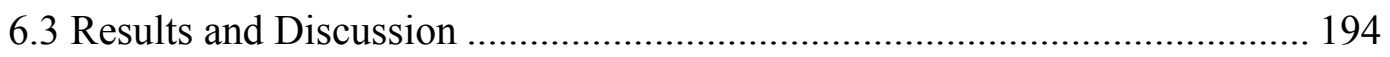

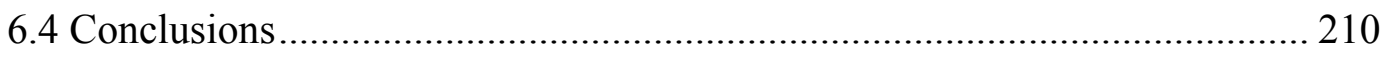

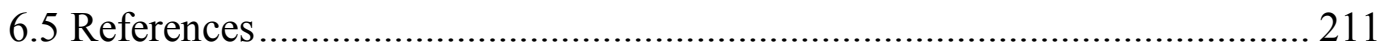

Chapter 7: Conclusions and Recommendations for Future Works............................ 215

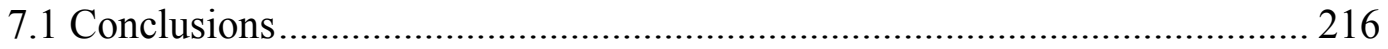

7.2 Recommendations for Future Work......................................................... 220

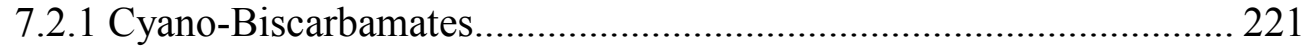

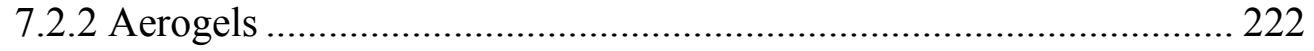

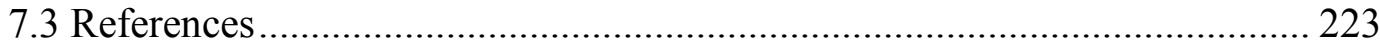

Appendix A $\quad$...................................................................................................................... 224

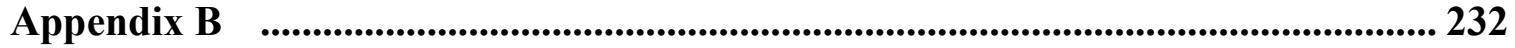




\section{LIST OF TABLES}

Table 2.1 Biscarbamates $\left(\mathrm{C}_{\mathrm{x}}\right)$ synthesized from reaction of an appropriate alcohol with 1,6-diisocyanato hexane...................................................................... 51

Table 2.2 Stretching frequencies of the IR bands of the representative $\mathrm{C}_{12}$ biscarbamate.

Table 3.1 Temperatures of dissolution and precipitation of various biscarbamates from chloroform. All the solutions were prepared using the same amount of substrate and solvent. 81

Table 3.2 Melting points of blends of biscarbamates prepared by solvent and melt mixing. 85

Table 3.3 Temperatures of dissolution and precipitation of various biscarbamates blends from chloroform. All the samples are prepared using the same amount of substrate and solvent............................................................88

Table 3.4 Bragg d-spacings of the peaks and the corresponding intensities (I(\%)) normalized with respect to the peak at $\mathrm{d}=3.6 \AA$ for $\mathrm{C}_{8} / \mathrm{C}_{12}$ solvent-based blend samples of various relative concentrations. 96

Table 3.5 Melting temperatures and the full-width-half-max (FWHM) of $\mathrm{C}_{8} / \mathrm{C}_{12}$ blends of biscarbamates prepared by solvent mixing and processed through solvent annealing in chloroform at room temperature. 106

Table 3.6 Melting points and the full-width-half-max of $\mathrm{C}_{8} / \mathrm{C}_{12} 50 / 50$ blend of biscarbamates prepared by solvent mixing and its improvements. The results indicate only single peaks. 108

Table 3.7 Melting points and the full-width-half-max of $\mathrm{C}_{4} / \mathrm{C}_{18} 50 / 50$ blends of biscarbamates prepared by solvent mixing and its improvements. The results indicate only single peaks. 110

Table 3.8 Melting points and the full-width-half-max of $\mathrm{C}_{12} / \mathrm{C}_{16} / \mathrm{C}_{18}$ melt blends of 
biscarbamates with varying $\mathrm{C}_{16}$ concentrations. The results indicate a single peak with varying width.................................................................................... 116

Table 4.1 The average lengths of the odd biscarbamate gels in benzonitrile at $0.08 \mathrm{M}$ prepared using the slow-cooled method.

Table 5.1 The critical gelation concentration for biscarbamate gels in benzonitrile. 162

Table 5.2 The gel-to-solution temperature $\left(\mathrm{T}_{\text {gel-sol }}\right)$ of short biscarbamate blend gels and their individual components with recorded using the DSC.

Table 5.3 The gel-to-solution temperature ( $\mathrm{T}_{\text {gel-sol }}$ ) of $\mathrm{C}_{8} / \mathrm{C}_{9}$ gel blends at various concentrations recorded using the DSC.

Table 6.1 The gel-to-solution temperatures for gels produced using both conventional heating and microwave heating methods, and the gelation temperature for the conventional heated gels. Due to limitation of the microwave apparatus, the gelation temperature cannot be determined for this method. 209 


\section{LIST OF ILLUSTRATIONS}

Figure 1.1 Representation of a tobacco mosaic virus with an RNS core and protein sheath, and of a reconstitution in vitro of TMV. 3

Figure 1.2 A representation of four equilibrium morphologies: spherical (S), cylindrical (C), gyroid (G) and lamellar, depending on the composition $f$ (overall volume fraction of the A component) and combination parameter $\chi N$ where $\chi$ is the A-B segment-segment (Flory-Huggins) interaction parameter and $N$ is the overall degree of polymerization. The colours represent the different nature of the composition of the block copolymer...

Figure 1.3 Schematic representation of a generic phase diagram for diblock copolymer blend. LAM, Hex, and BCC correspond to lamellar, hexagonal (cylindrical morphology) and body-centered-cubic (spherical morphology) symmetries...................................................................................6 6

Figure 1.4 Schematic representation of a) the pseudo network of LMOG; b) a chemically cross-linked network of polymers; c) entangled physical network of polymers. ............................................................................. 11

Figure 1.5 The primary, secondary, and tertiary structure of a self-assembled physical gel

Figure 1.6 Schematic progression of gelation under the conventional view. A sol of the gelator at high temperatures (a) is cooled, whereupon crystalline nuclei form (b). These then nucleate the growth of crystalline fibrils, which tend to be large (c), and the fibrils fill the volume to form a gel network (d).....

Figure 1.7 Schematic representation of Mono-PDMS and Di-PDMS molecules and SEM images of their respective xerogels made from propyl amine, hexanewater mixture or diisopropylamine-water mixture. 
Figure 1.8 Schematic representation of MJ-PTCDI and DJ-PTCDI molecules and SEM images of their respective xerogels showing honeycomb morphology. ... 19

Figure 1.9 Depiction of narcissistic and social self-sorting phenomena in multicomponent mixtures. 23

Figure 1.10 Conceptual representation of the sergeant-soldier effect when in presence of a chiral amplification on helical polymers.

Figure 1.11 A schematic representation of the sergeants-and-soldiers effect determining a preferred handedness of the helical stacks formed by achiral attached disc shaped molecules by doping them with a small amount of chiral molecule.

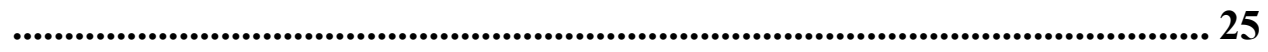

Figure 1.12 A schematic illustration of the induction of one-handed macromolecular helicity in dilute solution and the additional chiral amplification in the LC phase. 27

Figure 1.13 Pictorial representation of a $\mathrm{C}_{18}$ carbamate (top) and a $\mathrm{C}_{6}$ spacer biscarbamate (bottom). $\mathrm{R}$ is an alkyl side chain of length varying from $\mathrm{C}_{3}$ to $\mathrm{C}_{18}$ 28

Figure 1.14 Schematic showing the hydrogen bonding and van der Waals interaction in biscarbamates. 30

Figure 2.1 (Left) Image of an open ceramic cell where a sample vial is inserted, and a closed ceramic cell. (Right) Image of the microwave digestion apparatus with the reference cell connected to the instrument, and a sample cell.... 55

Figure 2.2 FTIR spectra of the $\mathrm{C}_{12}$ biscarbamate. 57

Figure 2.3 The spin- $-1 / 2$ nuclei include the most common ${ }^{1} \mathrm{H}$ NMR nucleus, as well as many other nuclei such as ${ }^{13} \mathrm{C},{ }^{15} \mathrm{~N}$ and ${ }^{31} \mathrm{P}$. 60

Figure 2.4 ${ }^{1} \mathrm{H}$ NMR spectra of $\mathrm{C}_{12}$ biscarbamate. ................................................ 61

Figure 2.5 Schematic representation of a DSC cell..................................................... 63 
Figure 2.6 Schematic presentation of a (a) positive spherulite, (b) negative spherulite, and (c) tint $\lambda$ plate. 65

Figure 2.7 Schematic diagram of a scanning electron microscope.............................. 68

Figure 2.8 Bragg construction illustrating the principle of diffraction where $d$ is the spacing between two atomic planes. 70

Figure 2.9 Schematic of the Bragg diffraction of the powder X-ray diffractometer used for the present study. Here, $\mathrm{S}_{1}, \mathrm{~S}_{2}$ and $\mathrm{S}_{3}$ are the divergence slit, receiving slit and scatter slit respectively; $\alpha=1-4^{\circ}$. 71

Figure 2.10 Schematic showing the crystalline and amorphous peak area used for calculating percent crystallinity. 72

Figure 3.1 The melting temperatures $\left(\mathrm{T}_{\mathrm{m}}\right)$ at various compositions and the miscibility curves for $\mathrm{C}_{4} / \mathrm{C}_{18}$ (a) melt and (b) solvent blends, $\mathrm{C}_{8} / \mathrm{C}_{12}$ (c) melt and (d) solvent blends, and (e) $\mathrm{C}_{16} / \mathrm{C}_{18}$ melt and solvent blends. Both $\mathrm{C}_{4} / \mathrm{C}_{18}$ and $\mathrm{C}_{8} / \mathrm{C}_{12}$ blends have multiple melting points (endotherms). 86

Figure 3.2 Variation of $\Delta \mathrm{T}_{\mathrm{m}}$ for (a) $\mathrm{C}_{4}$ and (b) $\mathrm{C}_{18}$ for the $\mathrm{C}_{4} / \mathrm{C}_{18}$ blend, (c) $\mathrm{C}_{8}$ and (d) $\mathrm{C}_{12}$ for the $\mathrm{C}_{8} / \mathrm{C}_{12}$ blend, and (e) $\mathrm{C}_{16}$ and (f) $\mathrm{C}_{18}$ for the $\mathrm{C}_{16} / \mathrm{C}_{18}$ blend with addition of the other component. There is a larger change in $\Delta \mathrm{T}_{\mathrm{m}}$ for the melt blends compared to the solvent-based blends. . 90

Figure 3.3 DSC traces of $\mathrm{C}_{8} / \mathrm{C}_{12}$ biscarmabates blends at various concentrations: (a) 90/10, (b) 75/25, (c) 60/40, (d) 50/50, (e) 40/60, (f) 25/75, and (g) 10/90. The peaks become narrower as the relative concentrations of the components become comparable. In the cases of (a), (b) and (e), TA Universal Analysis software could not identify the location of the shoulder peak. The values of the peaks were then obtained using a model fit by Igor Pro

Figure 3.4 Model of domain mix versus molecular mix. 95

Figure 3.5 (a) Normalized XRD patterns of $\mathrm{C}_{8} / \mathrm{C}_{12}$ biscarbamate blends obtained from 
solvent mixing at various concentrations. The peaks are in the same positions, but the widths and height differ. This is an indication of the change in crystallinity of the blends. (b) Percent crystallinity of $\mathrm{C}_{8} / \mathrm{C}_{12}$ solvent-based blend showing a change in crystallinity as a function of concentration 98

Figure 3.6 Optical micrographs of (a) $\mathrm{C}_{8} / \mathrm{C}_{12}: 10 / 90$ at $100{ }^{\circ} \mathrm{C}$ and (b) $\mathrm{C}_{8} / \mathrm{C}_{12}: 50 / 50$ at $103{ }^{\circ} \mathrm{C}$. 99

Figure 3.7 Optical micrographs of biscarbamate $\mathrm{C}_{8} / \mathrm{C}_{12}(50 / 50)$ prepared by solventmixing taken various time frames during spherulite growth. 101

Figure 3.8 Optical micrographs of individual biscarbamates $\mathrm{C}_{8}(\mathrm{a})$ and $\mathrm{C}_{12}(\mathrm{~b})$, and blends at concentration 25/75 (c), 50/50 (d) and 75/25 (e) crystallized from the solvent as the temperature is kept at $8^{\circ} \mathrm{C}$. 102

Figure 3.9 (top) Variation of spherulite size with time for $\mathrm{C}_{8}, \mathrm{C}_{12}$ and their blends when crystallized from solution (average of three experiments) and (bottom) the rate of spherulite growth as a function of wt $\% \mathrm{C}_{8}$ added to the $\mathrm{C}_{8} / \mathrm{C}_{12}$ solution blend. 104

Figure 3.10 DSC thermographs of various concentrations of $\mathrm{C}_{8} / \mathrm{C}_{12}$ blends prepared by thermal annealing. 107

Figure 3.11 DSC endotherms of $\mathrm{C}_{8} / \mathrm{C}_{12}$ biscarbamate (a) $50 / 50$, (b) $25 / 75$ and (c) $75 / 25$ blends prepared by various methods. 109

Figure 3.12 Melting points of $\mathrm{C}_{3} / \mathrm{C}_{6}$ biscarbamate blends at (a) $50 / 50$, (b) $25 / 75$ and (c) $75 / 25$ mixture prepared by solvent mixing and melt blend. There is a shoulder on the melt blend of the 50/50 blend (a) thermograph that is not present for the solvent mixing sample. This shoulder is an indication of the sample not fully mixing. The 25/75 (b) and 75/25 (c) blends show multiple peaks for both the solvent- and melt-based blends. 113

Figure 3.13 DSC thermographs of 3 component blends. 115 
Figure 3.14 DSC thermographs of 3 component blends for $\mathrm{C}_{4} \mathrm{C}_{\mathrm{x}} \mathrm{C}_{18}$.

Figure 4.1 The minimum concentration $\left(\mathrm{C}_{\min }\right)$ required for gelation of biscarbamates in benzonitrile. N.B. $\mathrm{C}_{14}$ and $\mathrm{C}_{17}$ biscarbamate were never synthesized due to the limited availability of the reagents.

133

Figure 4.2 Gelation time versus alkyl chain length for both odd and even biscarbamates. Model curves to illustrate the trends were calculated using Igor Pro.

Figure 4.3 DSC thermograph of $\mathrm{C}_{13}(0.08 \mathrm{M})$ wet gel in benzonitrile, heating at a rate of $10{ }^{\circ} \mathrm{C} / \mathrm{min}$ (pausing at $90{ }^{\circ} \mathrm{C}$ for 30 minutes), cooling at a rate of 10 ${ }^{\circ} \mathrm{C} / \mathrm{min}$ (pausing at $20{ }^{\circ} \mathrm{C}$ for 30 minutes) and heating again at a rate of $10^{\circ} \mathrm{C} / \mathrm{min}$. The heat of fusion for the first endotherm is $40.18 \mathrm{~J} / \mathrm{g}$ and the heat of fusion of the second endotherm is $38.84 \mathrm{~J} / \mathrm{g}$ 135

Figure 4.4 The melting temperature of $0.08 \mathrm{M}$ biscarbamate gels and of the respective crystals. N.B. $\mathrm{C}_{14}$ and $\mathrm{C}_{17}$ biscarbamate were never synthesized due to the limited availability of the reagents. 136

Figure 4.5 SEM images of $\mathrm{C}_{9}$ biscarbamate ( $\mathrm{a}$ and $\mathrm{b}$ ) quenched (low and high magnification) and (c and d) slow-cooled (low and high magnification) $(0.08 \mathrm{M})$ prepared from benzonitrile. 138

Figure 4.6 SEM images of xerogels of (a) $C_{7}$, (b) $C_{9}$, (c) $C_{11}$, (d) $C_{13}$ and (e) $C_{15}$ $(0.08 \mathrm{M})$ prepared from benzonitrile. All samples were slow-cooled, dried overnight and then left in the vacuum oven for a day. 140

Figure 4.7 SEM images of xerogels of ( $a$ and b) $\mathrm{C}_{8}$ and (c and d) $\mathrm{C}_{9}(0.08 \mathrm{M})$ prepared from benzonitrile. Both samples were slow-cooled, dried in the fumehood overnight and in the vacuum oven for a day 142

Figure 4.8 Low (left) and high (right) resolution cryo-SEM images of wet $\mathrm{C}_{7}$ gels prepared using the slow-cooling method after (a) 1 day, (b) 1 week, and (c) 2 weeks after formation. 145 
Figure 4.9 Low (left) and high (right) resolution cryo-SEM images of wet $\mathrm{C}_{7}$ gels prepared using the quenching method after (a) 1 day, (b) 1 week, and (c) 2 weeks after formation.

Figure 5.1 Phase diagram of biscarbamate blends at 50/50 $\mathrm{C}_{\mathrm{x}} / \mathrm{C}_{\mathrm{y}}$ in benzonitrile. Shorter biscarbamate require longer biscarbamate in order to for a gel blend. 161

Figure 5.2 Endothermic peaks of biscarbamate gels of $\mathrm{C}_{15}(0.04 \mathrm{M})$ and $\mathrm{C}_{3} / \mathrm{C}_{15} 50 / 50$ $(0.04 / 0.04 \mathrm{M})$ mixture in benzonitrile. There is a shift due to the presence of $\mathrm{C}_{3} . \mathrm{C}_{3}$ is incapable of gelling by itself even at 4 times the concentration. 163

Figure 5.3 DSC thermographs of the gel-sol and sol gel transitions of $50 / 50 \mathrm{C}_{3} / \mathrm{C}_{15}$ showing thermoreversibility. 165

Figure 5.4 SEM images of xerogels of biscarbamate combination $50 / 50 \quad \mathrm{C}_{3} / \mathrm{C}_{15}$ prepared by (a) quenched and (b) slow-cooled methods. 166

Figure 5.5 Optical micrographs of (a) $C_{3}$ in benzonitrile, (b) $C_{15}$ gel and (c) $C_{3} / C_{15}$ $80 / 20$ gel blend. 168

Figure 5.6 SEM images of xerogels of biscarbamate combination $\mathrm{C}_{8} / \mathrm{C}_{9}$ at concentration ratios of (a) 0/100, (b) 50/50, (c) 80/20, (d) 90/10, and (e) 100/0. 170

Figure 5.7 Optical microscope images of wet gels of biscarbamate 50/50 combination of $\mathrm{C}_{8} / \mathrm{C}_{9}$ prepared by (a) quenched and (b) slow-cooled methods. 171

Figure 5.8 SEM images of wet gels of biscarbamate combinations $50 / 50 \mathrm{C}_{7} / \mathrm{C}_{11}$ prepared by quenched (a) and slow-cooled (b) methods. 174

Figure 5.9 Optical microscope images of wet gels of biscarbamate 50/50 combination of $\mathrm{C}_{7} / \mathrm{C}_{11}$ prepared by (a) quenched and (b) slow-cooled methods. 174

Figure 5.10 Low and high resolution SEM images of xerogels of biscarbamate 
combination $\mathrm{C}_{7} / \mathrm{C}_{11}$ at concentration ratios of (a) 100/0, (b) $90 / 10$, (c) $80 / 20$, (d) 50/50, (e) 20/80. (f) 10/90, and (g) 0/100. 177

Figure 5.11 DSC thermographs showing the gel-sol, sol gel transitions of $\mathrm{C}_{7} / \mathrm{C}_{11}$. The individual peaks represent the endotherms of the individual component. It would appear that they are not miscible in the gels level. 178

Figure 5.12 SEM and OM images of wet gels of biscarbamate combination $50 / 50 \mathrm{C}_{7} / \mathrm{C}_{9}$ prepared by quenched (left) and slow-cooled (right) methods. 180

Figure 5.13 DSC thermographs showing the gel-sol, sol-gel transitions of $\mathrm{C}_{7} / \mathrm{C}_{9}$. This does not seem reversible as the first endotherm shows one peaks, and the ones in the following cycles show two peaks.

182

Figure 6.1 Change in the minimum gelation concentration as a function of number of carbon atoms in the alkyl side chains using the hot plate and the microwave system. 196

Figure 6.2 Change in the dissolution temperature of the $0.08 \mathrm{M}$ biscarbamate gel in benzonitrile made using a hotplate or a microwave system as a function of number of carbon atoms in the alkyl side chains.

Figure 6.3 Minimum dissolution temperature for $\mathrm{C}_{9}$ biscarbamate gels in various solvents using (a) conventional and (b) microwave heating, for a range of gelator concentrations starting with the critical gelation concentration. The dipole moments of the solvents are listed in the insets. 201

Figure 6.4 (a) SEM and (b) OM images of benzonitrile/C9 based gels using conventional heating. (c) SEM and (d) OM images of gels made using MW source. 203

Figure 6.5 X-ray diffractogram of $\mathrm{C}_{9}$ biscarbamate dried gel. As opposed to xerogels, the dried gel was attained by drying off the excess solvent and leaving the slide exposed to air for 1 hour which would limit the appearance of an amorphous band. The designation "parallel' denotes the sample orientation 
along the direction of the incident X-ray beam and "perpendicular" denotes the sample rotated by $90^{\circ}$

Figure 6.6 (a) Low and (b) high magnification SEM images of $\mathrm{C}_{12}$ biscarbamate xerogels from benzonitrile made using a hot plate and (c) low and (d) high magnification SEM images of xerogels made using a microwave digestive system. 206

Figure 6.7 SEM images of $\mathrm{C}_{9}$ biscarbamate xerogels from (a,b) DMSO, (c,d) 1octanol, (e,f) xylene, and (g,h) toluene made using (left) a hot plate and (right) a microwave digestive system. 208 
CHAPTER 1

Introduction 


\section{CHAPTER I: INTRODUCTION}

\subsection{Molecular Self-Assembly}

Molecular self-assembly is the spontaneous association of molecules under equilibrium conditions into stable, structurally well-defined aggregates joined by noncovalent bonds or weak covalent interactions (van der Waals, electrostatic, and hydrophobic interactions, hydrogen and coordination bonds) as opposed to strong covalent bonds. Weak noncovalent interactions will yield a self-assembly processes involving reversible formations. The reversible nature of the self-assembly processes leads to a preferred pathway which is self-checking and self-correcting. As a result, defective subunits are eliminated from the growing superstructure, ensuring a high degree of control. $^{1}$

Molecular self-assembly is ubiquitous in biological systems (e.g. DNA and RNA) and underlies the formation of a wide variety of complex biological structures. ${ }^{1}$ In fact, one of the first studies of the dynamic and reversible nature of self-assembly originates from the study of the tobacco mosaic virus and the enzyme ribonuclease. ${ }^{2-5}$ The tobacco mosaic virus (TMV) is a helical virus particle composed of 2130 identical subunits, each composed of 158 amino acids, which form a helical sheath around a single strand of RNA (Figure 1.1). It was observed that TMV could be dissociated into its component parts, ${ }^{4}$ and the isolated components reconstituted in vitro, re-forming the intact virus. The reassembly process exhibited concentration, time, and $\mathrm{pH}$ dependencies characteristic of a typical chemical reaction. ${ }^{4}$ This reconstitution experiment showed that all the information necessary to faithfully assemble the virus is built into the constituent parts. ${ }^{5}$ 


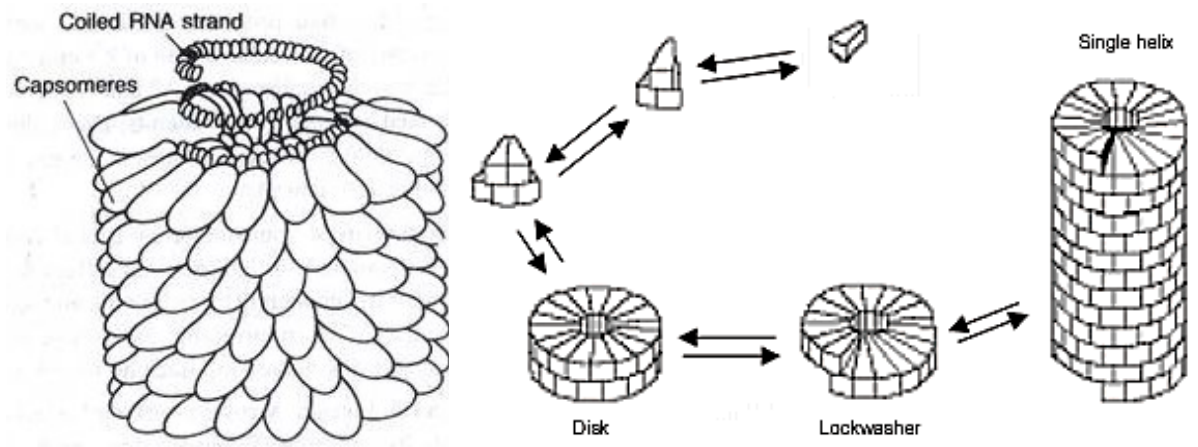

Figure 1.1. Representation of a tobacco mosaic virus with an RNS core and protein sheath, and of a reconstitution in vitro of TMV (taken from reference 4,5).

Inspiration from nature is of particular importance for designing functional building blocks towards self-assembly. Nature-inspired molecular self-assembly has thus emerged as a new approach in chemical synthesis, nanotechnology, polymer science, materials science and engineering. For instance, self-assembled nanomaterials can be effectively applied in the field of regenerative medicine, pharmacotherapeutics, and medical health care to discover novel drugs, drug delivery systems, imaging, and gene therapy. Selfassembly, electrospinning, and phase separation are three different approaches toward formation of nanofibrous materials. ${ }^{6}$ Fabricated materials by each method are very different in terms of physical and chemical characteristics, which lends to its development and biological applications.

Self-assembly in the context of materials implies that the components are programmed by design to create a functional, ordered structure with little intervention from humans or machines. This process can occur at any scale, ranging from the nano- and micrometer scale to macroscopic dimensions. By far the most popular research in soft 
matter has been attempting to design relatively simple structures using hydrogen bonding, $\pi-\pi$ stacking, metal-ligand interactions, strong dipole-dipole association, hydrophobic forces, and steric repulsion. The reality is that our ability to predict the structural features from combinations of such interactions, let alone the functional outcomes, in the assembled materials is still in its infancy and in these early times for self-assembly and supramolecular chemistry most of the learning is done in retrospect after discovery of structures and their functions. The environment can modify the interactions between the components; the use of boundaries and other templates in self-assembly is fairly common, because templates can reduce defects and control structure formation. ${ }^{7}$ Equilibration time is usually required to reach ordered structures. If components stick together irreversibly when they collide either by covalent bonds or strong H-bonding, they form a glass rather than a crystal or other regular structure. Defect free structures come from the fact of having a dynamic system with weak interactions which allows for reorganization to get well-ordered structures.

One of the most widely used materials for the production of self-assembled nanomaterials are amphiphilic (both hydrophilic and hydrophobic) block copolymers. Block copolymers comprise of two or more homopolymer subunits linked by covalent bonds (e.g. AAAAAA-BBBBB, where A and B are different polymer subunits). Their defect-free structure comes from the fact of having a dynamic system with weak interactions which allows for reorganization to get well-ordered structures. Their applications range from foam stability to drug delivery ${ }^{8}$ and nanowire templates. ${ }^{9}$ The association, variety of shapes and properties of block copolymers have been extensively characterized. $^{8}$ 


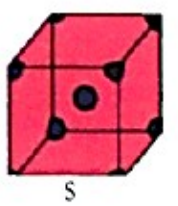

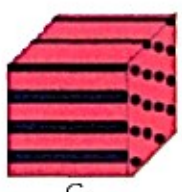

C

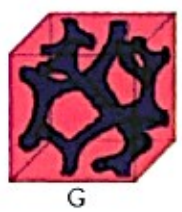

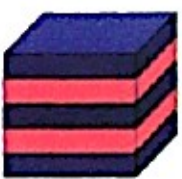

L

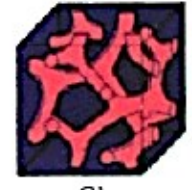

G'

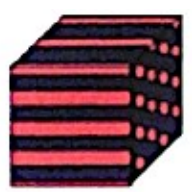

C'

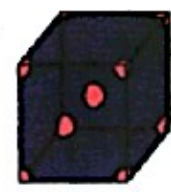

S'

Figure 1.2. A representation of four equilibrium morphologies: spherical (S), cylindrical $(\mathrm{C})$, gyroid $(\mathrm{G})$ and lamellar $(\mathrm{L})$, depending on the composition $f$ (overall volume fraction of the A component) and combination parameter $\chi N$ where $\chi$ is the A-B segment-segment (Flory-Huggins) interaction parameter and $N$ is the overall degree of polymerization. ${ }^{10}$ The colours represent the different nature of the composition of the block copolymer. Adapted from ref 10.

For instance, the Pluronic triblock copolymer composed of one central poly(propylene oxide) (PPO) block connected to two poly(ethylene oxide) (PEO) blocks was observed to form unimers, spherical micelles (Figure 1.2S), cylindrical micelles (Figure 1.2C), gyroid vesicles (Figure 1.2G) and well-order lamellar sheets (Figure 1.2L) depending on the temperature and copolymer concentration. ${ }^{9}$ The careful characterization of the structure in response to external factors (i.e. concentration, temperature, nature of the solvent) allowed the preparation of very well defined ordered nanostructures (Figure 1.3). These types of Pluronics are commercially available materials used in cosmetics and pharmaceutical industries. ${ }^{11}$ The influence of self-assembly strategies in polymeric materials research is reflected in the strong interest on the organization of well-defined block copolymers into defined superlattices and patterns. ${ }^{12}$ Most notably the exciting new field of supramolecular polymers ${ }^{13}$ is based on control of noncovalent interactions among 
monomers and processes of self-assembly to generate ordered 1D structures. In organic nanoscience supramolecular chemistry is key in the design of self-assembling monolayers, ${ }^{14}$ as well as nanostructures with diverse shapes and dimensionalities. ${ }^{15}$ Supramolecular chemistry has enabled the development of bulk materials such as molecular organic frameworks, ${ }^{16}$ organogels,${ }^{17}$ and biomolecular materials for medicine based on peptides. ${ }^{18}$

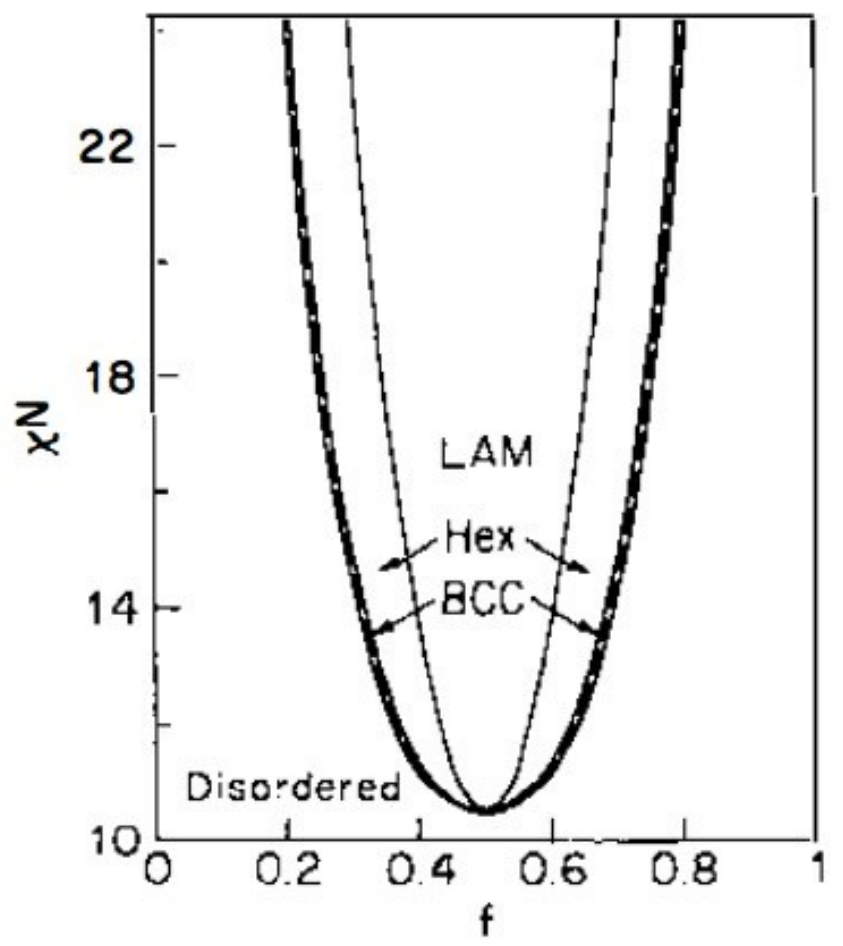

Figure 1.3. Schematic representation of a generic phase diagram for diblock copolymer blend where $f$ is the overall volume fraction of the A component for a A-B segmentsegment copolymer. LAM, Hex, and BCC correspond to lamellar, hexagonal (cylindrical morphology) and body-centered-cubic (spherical morphology) symmetries. Taken from ref 10. 
Some applications obtained from the self-assembly of block copolymers include nanocarriers of diagnostic and therapeutic agents. ${ }^{19}$ The dissolution of an amphiphilic block copolymer in a solvent that is only "good" for one block, leads to an attractive and a repulsive force that results in microphase separation and self-assembly of individual molecules into spherical micelles. ${ }^{19}$ The intended drug is either entrapped in the hydrophobic core of the micelle during its formation or covalently bound to the hydrophobic block in such formulations. ${ }^{19}$ Chilkoti and co-workers have reported the fabrication of multivalent spherical micelles from stimuli responsive elastin-like polypeptides for drug targeting by thermally triggered multivalency. ${ }^{20,21}$ Micelles have been also used in anticancer drug carrier to probe into the cancer cell apoptosis. ${ }^{22}$

Molecular self-assembly has evolved as a highly promising field of contemporary research and the above mentioned examples are only a few of the immense possibilities that molecular self-assembly can offer. It has now become evident that molecular selfassembly will surely be the most important designer toolkit in the coming decades. ${ }^{23}$ The various types of material resulted from self-assembly that will be discussed in this thesis include gels and blends.

\subsection{Gels}

A relatively large number of reviews, ${ }^{24-28}$ as well as some books, ${ }^{29,30}$ have been devoted to aspects of gels. More than 80 years ago, Dorothy Jordon Lloyd stated for all gel types that "...the colloid condition, the gel, is easier to recognize than to define. ${ }^{31}$ " Although Lloyd's statement seemed enigmatic, it is true even today because the 
composition and structure of the matrix of a viscoelastic material, as well as its rheological properties, must be considered when properly defining a gel whether it be molecular or any other type.

Gels have pervaded our everyday life in a variety of forms. The wet soft solids that we encounter in the form of commercial products such as soap, shampoo, toothpaste, hair gel and other cosmetics, as well as contact lenses and gel pens etc. are all gels derived from polymeric compounds. Polymer gels have been known for centuries, and applications in fields as diverse as food, medicine, materials science, cosmetics, pharmacology, sanitation etc. have been realized for these systems. ${ }^{27}$ Here, gels and their properties will be discussed in further detail.

\subsubsection{Supramolecular Gels vs. Polymer Gels}

A polymer gel consists of an elastic polymer 3D network with a fluid filling the interstitial space of the network. The network of polymer molecules holds the liquid in place and so gives the gel what rigidity it has. There are a variety of ways to classify gels, such as natural gel or synthetic gel, according to the source; hydrogel or organogel, according to the liquid medium in the polymer network; and chemical or physical gels, according to their cross-linkage. The classification of the cross-linkage is a property unique to polymer gels.

A polymer gel is a network of flexible chains. Structures of this type can be obtained by chemical or physical processes. Some polymer gels are cross-linked chemically by covalent bonds (chemical gel), whereas other gels are cross-linked physically by polymer chain entanglements (physical gel). The final gel structures and properties are sensitive to 
the preparation methods.

For chemical gels, one approach to gel synthesis utilises additive polymerization (Figure 1.4b). For example, in the case of a vinyl monomer, the double bond can be reacted by a free radical reaction resulting in mainly linear chains. However, if a fraction of a divinyl derivative was added to the mixture $\mathrm{CH}_{2}=\mathrm{CH}-\mathrm{R}_{2}-\mathrm{CH}=\mathrm{CH}_{2}$, the two double bonds will participate in the construction of two distinct chains, and $-\mathrm{R}_{2}-$ will become a crosslinking bridge in the structure. ${ }^{32}$ A second method of gel preparation is based on the condensation of polyfunctional units. A typical example would be the condensation reaction between a trialcohol and diisocyanate. The reaction leads to branched chains; each trialcohol becoming a branch point when its three functions are reacted. These polymer networks swell in appropriate solvents, but do not dissolve. The degree of swelling strongly depends on the degree of cross-linkage. The lower the degree of cross-linkage, the more the gel swells.

The polymer network of physical gels consists of several components including bridging strands, cross-links (or junctions), dangling ends, and loops that trap the solvent (Figure 1.4c). Physical gelation in polymers is considered to be the result of liquid-liquid phase separation and vitrification caused by the spinodal decomposition mechanism. ${ }^{33,34}$ Such a phenomenon could also be accompanied by crystallization in the case of semicrystalline polymers. ${ }^{26}$ Gels with crystallizable polymers such as isotactic polystyrene, poly(vinyl chloride), and polyethylene have been studied, ${ }^{35-41}$ and liquid-liquid phase separation accompanied by crystallization has been attributed to the formation of networks. However, non-crystallizable polymers have been shown to form physical gels as well which suggests that the mechanism of physical cross-linking that produces the gel network 
in non-crystalline polymers is an equilibrium process that does not involve crystallization in the conventional sense. The gel of atactic polystyrene (a-PS) ${ }^{42}$ which is a noncrystallizable polymer, has also been found to form networks or spherical domains by networks with cyclohexanol as the solvent. Atactic polystyrene also undergoes thermoreversible gelation. ${ }^{42}$

Gels derived from low molecular mass organic gelators ("supramolecular gels" or LMOGs), although known for a long time, have started to be actively investigated only during the past fifteen years, and applications as diverse as those realized for their polymeric counterparts have been envisaged for these systems. ${ }^{26,30}$ The network of these gels is formed by the self-assembly process (hydrogen bonding, $\pi-\pi$ stacking, van der Waals interactions etc.), Recent years have witnessed a surge of research work dedicated to LMOGs because of their various potential applications in sensors, ${ }^{43}$ electrooptics/photonics, ${ }^{44}$ structure directing agents, ${ }^{45}$ cosmetics ${ }^{46}$ conservation of arts, ${ }^{29}$ drug delivery and bio-medical applications $\mathrm{s}^{22,23,27,30}$ etc. Although a wide range of gelators has been reported, it is unfortunate that, so far, the development of new gelators is more a serendipity than a planned event. ${ }^{47}$

Physical polymer gels and LMOGs are made up of different building blocks (Figure 1.4a), but they are very similar as far as morphology and rheological properties are concerned. Both systems are thermoreversible which means they can be melted and reformed at will through heating and cooling cycles without altering the chemical structures of their constituents. In general, gels are viscoelastic solid-like materials comprised of an elastic cross-linked network and a solvent, which is the major component. The solid-like appearance of a gel is a result of the entrapment and adhesion of the liquid 
in the large surface area of the solid 3D matrix. LMOGs, small organic compounds having molecular mass typically $<3000$ Da strictly belong to the supramolecular gelator category and are amazingly powerful in immobilizing organic solvents (organogels) and pure water and/or aqueous solvents (hydrogels) at very low concentration of the gelator. ${ }^{25-28}$ These physical gels obtained from LMOGs are generally thermoreversible (reversible sol-gel transformation upon heating and cooling) and in some rare occasions display thixotropic (reversible sol-gel transformation under mechanical stress followed by rest) behaviour. ${ }^{48}$ LMOGs which are able to gel organic liquids, generally do not display hydrogelling capability and vice versa. For the purposes of this thesis, we will mainly focus on LMOGs.

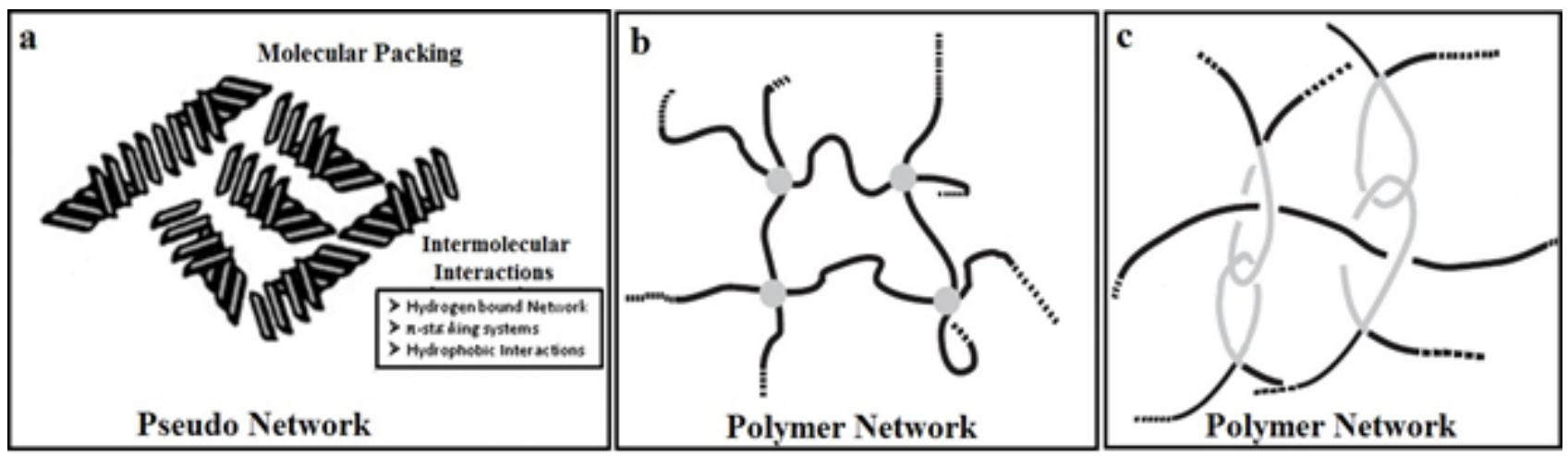

Figure 1.4. Schematic representation of a) the pseudo network of LMOGs; b) a chemically cross-linked network of polymers; c) entangled physical network of polymers.

\subsubsection{The Principles of Gelation for Small Organic Molecules}

Flory defined a gel as a two-component colloidal dispersion, with a continuous structure with macroscopic dimensions that is permanent on the time scale of the 
experiment and is solid-like in its rheological behaviour. ${ }^{49}$ Usually, gels are formed by dissolving a small amount (usually 0.1-10 wt \%) of gelator in hot solvent (water for hydrogels). ${ }^{49}$ Upon cooling below the $\mathrm{T}_{\text {gel }}$ (temperature of gelation), the complete volume of solvent is immobilized and can support its own weight without collapsing (often tested by turning the test tube upside down; if no flow is observed, the solution is said to have gelled).

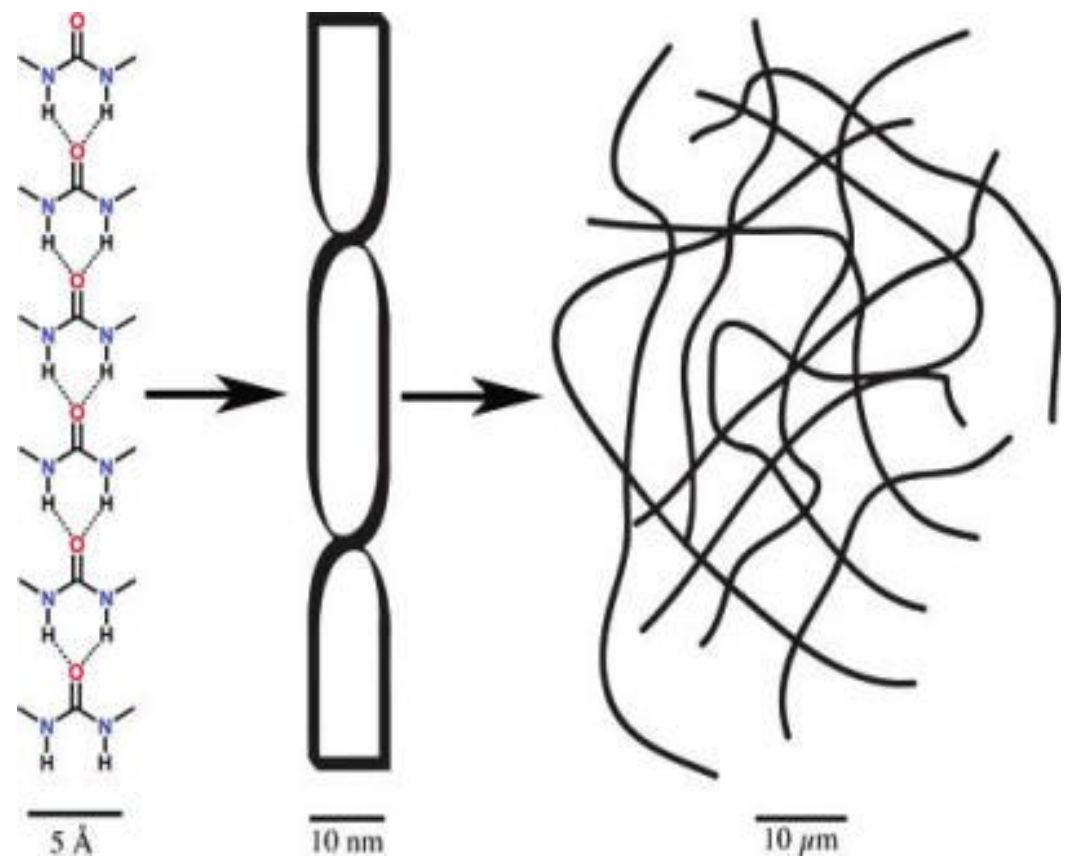

Figure 1.5. The primary, secondary, and tertiary structure of a self-assembled physical gel. Taken from ref 25.

The phenomenon of gelation is thought to arise from fibres (nano- to micrometer) becoming entangled and trapping solvent via surface tension. ${ }^{49}$ To understand the 
mechanism of gel formation, a gel can be broken down into a primary, secondary, and tertiary structure, much like a protein (Figure 1.5). ${ }^{25}$ The primary structure (angstrom to nanometer scale) is determined by the molecular level recognition events that promote anisotropic aggregation in one or two dimensions of the gelator molecules. Assembly of small organic molecules in aqueous solvents into fibrous structures poses interesting challenges in the fields of molecular recognition and self-assembly. To achieve gelation, there must be a balance between the tendency of the molecules to dissolve or to aggregate. Hydrogen bonds, a common driving force for aggregation in organogelators, lose their strength in water unless many are combined in a cooperative manner and protected from solvent. ${ }^{50}$ Instead, hydrophobic forces, which lack the precise directing ability of hydrogen bonds, become most important in aqueous environments. ${ }^{51}$

The secondary structure (nano- to micrometer scale) is defined as the morphology of the aggregates, that is, micelles, vesicles, fibres, ribbons, or sheets, and is directly influenced by the molecular structure (Figure 1.5). Multiple morphologies are observed, ${ }^{25}$ including micelles, vesicles, lamellae, and amorphous or crystalline precipitates. Micelles are fluid species and form at the critical micellar concentration (CMC), which depends on the structure of the amphiphile. Above this concentration, micelles can convert to ellipsoidal micelles (disks) and then, with further increase in concentration, to cylindrical micellar fibres (rods). These fibres, however, generally precipitate or display viscoelastic behaviour at concentrations above the $\mathrm{CMC}$ without forming a gel, due to electrostatic repulsion of the charged surfaces. ${ }^{24}$ These aggregates exist along with the gel state as part of a continuum controlled by $\mathrm{pH}$, temperature, ionic strength, and other factors.

Finally, the tertiary structure of a gel (micro- to millimeter scale) involves the 
interaction of individual aggregates and ultimately determines whether a gel is formed or, instead, fibres (or other aggregates) precipitate from solution rather than trap it (Figure 1.5). The transition from secondary to tertiary structure is determined by the type of interactions that can occur among the fibres. Physically, long, thin, flexible fibres are better able than shorter fibres to trap solvent, leading to gelation. ${ }^{49}$ The experimental conditions can be varied to achieve different morphologies and, thus, gels with different physical properties. For example, Liu, Sawant, and co-workers have demonstrated how the presence of an additive can promote branching and, thus, gelation. ${ }^{52-54}$ In other systems, the gelation temperature ${ }^{54}$ and rate of cooling ${ }^{55}$ have been found to alter the properties of the resulting gels. Presumably, such variables affect the rates of nucleation and growth, with an increasing number of branching events at higher temperatures.

Unlike polymer gels, in which the basic elements of a 3D network are onedimensional (1D) objects, molecular gels are composed of zero-dimensional (0D) objects on the micrometer scale that self-assemble through noncovalent interactions into 1D objects; rarer are molecular gels in which the self-assembly is $0 \mathrm{D} \rightarrow 2 \mathrm{D}$ (i.e., platelets) $\rightarrow 3 \mathrm{D}$ objects. ${ }^{56}$ As a result of the nature of the intermolecular interactions among the gelator molecules as well as among the gelator molecules and the liquid component, these gels are almost always thermally reversible with their sol phases. The sols consist of individual gelator molecules or their aggregates without a continuous network. An important exception to the reversibility is when heating or otherwise perturbing the sol phase results in chemical changes to at least one of the gel components. 


\subsubsection{Properties of Supramolecular Gels}

A gel is usually identified on the basis of its ability to withstand its weight under gravity in an inverted vial. The material is "solid-like" in its rheological behaviour: ${ }^{49}$ the storage modulus $\left(\mathrm{G}^{\prime}\right)$ must remain larger than the loss modulus $\left(\mathrm{G}^{\prime \prime}\right)$ over a large frequency range (including low frequencies) within the linear viscoelastic region; the mechanical strength of a gel is related to the absolute magnitudes of the moduli and their ratio, $\mathrm{G}^{\prime} / \mathrm{G}^{\prime \prime}{ }^{29}$ Whereas the yield stress and frequency-independent moduli are common to all gels, one striking feature of gels based on crystalline fibrils is that their strain limit tends to be very low; strains of $\sim 1$ to $2 \%$ are sufficient to disrupt the gel. ${ }^{29}$ Additionally, when much larger strains or shear rates are applied, the gel is often irreversibly disrupted and converted to a sol (or in other cases, the gel recovers very slowly, over many hours). ${ }^{29}$

The development of a typical gel from small molecule gelators under the "conventional" methods is depicted in Figure 1.6. To begin, the gelator is dissolved in the solvent of interest at high temperature. The resulting sol (Figure 1.6a) is typically a clear, homogeneous solution of low viscosity. When the sol is cooled below a characteristic supersaturation temperature, nuclei of nano/microscale dimensions arise in the sample (Figure 1.6b). With time, these nuclei usually grow into straight or branched fibrils (Figure 1.6c), and the overlap or entanglement of these fibrils ultimately leads to gelation (Figure 1.6d). Studies on such gels using X-ray diffraction (XRD) usually reveal crystalline peak(s), indicating that the fibrils are crystalline. ${ }^{57}$ Often, corresponding peaks are found in the powder XRD pattern of the gelator itself, which implies that the packing of gelator molecules in fibrils is analogous to that in its crystals. ${ }^{57}$ The presence of fibrils/spherulites can also be directly detected in typical cases by optical microscopy, which means that the 
fibrils are quite large (diameters on the order of hundreds of nanometers; lengths on the order of tens of micrometers). ${ }^{57}$

(a) Sol (at high T)

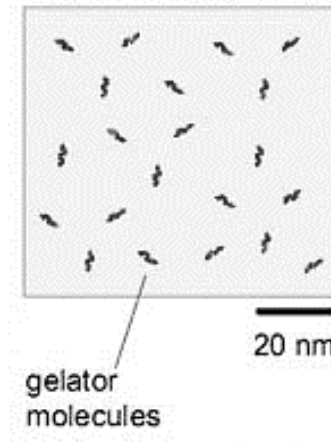

(b) Nucleation

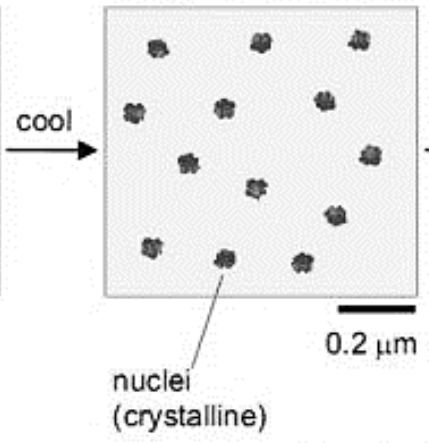

(c) Growth of Fibrils

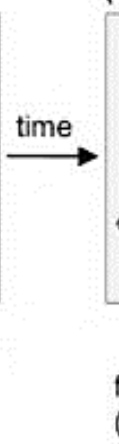

(d) Gel (Network)

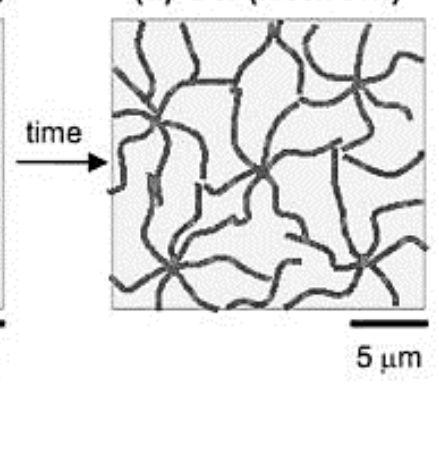

Figure 1.6. Schematic progression of gelation under the conventional view. A sol of the gelator at high temperatures (a) is cooled, whereupon crystalline nuclei form (b). These then nucleate the growth of crystalline fibrils, which tend to be large (c), and the fibrils fill the volume to form a gel network (d). Figure adapted from ref 58.

Gels can be easily transformed into solution by heating them and back to gel by cooling the solution above and below their sol-gel transition temperature. ${ }^{49}$ This reversible property of the gels can be used to make matrices for applications such as controlled release of drugs, fragrance, photonic materials, catalysts, and is useful in crystal and fuel engineering. ${ }^{27,28}$ A low concentration is sufficient for gel formation, and network filaments (length up to few micrometers) are formed by well-ordered arrays of molecules.

Gelator molecules self-assemble by highly specific noncovalent interactions, leading to various structures, which form a 3D network. Therefore, self-complementary 
and unidirectional intermolecular interactions can be considered a prerequisite for the gelation ability. The intermolecular interactions between the organogelator molecules can be hydrogen bonding, London dispersion forces, electrostatic attractions, and $\pi-\pi$ stacking. For example, our group has looked at supramolecular gels consisting of perylene imides (PDI) substituted with poly(dimethylsiloxane) (PDMS) either on one imide position (Mono-PDMS) or on both positions (Di-PDMS), ${ }^{59-62}$ and substituted with Jeffamine ${ }^{\circledR}$ on one imide nitrogen (MJ-PTCDI) or both (DJ-PTCDI). ${ }^{63,64}$ It was discovered that of monosubstituted PDI (Mono-PDMS) gels consist of hollow spheres while di-substituted PDI (Di-PDMS) gels consist of ribbons that are folded along the length into an eaves through (Figure 1.7). The gelation was attributed by the entanglement of the PDMS, in addition to the $\pi$-interaction mediated self-assembly of the perylene segments. Morphological studies showed that although fibres are not seen for DJ-PTCDI gels, the "chaining" of the spheres seems to be present. The SEM image of the cross section of the MJ-PTCDI sample shows the honeycomb morphology similar to that of DJ-PTCDI (Figure 1.8). Thus with MJPTCDI based gels also, the worm-like vesicular morphology occurs. 


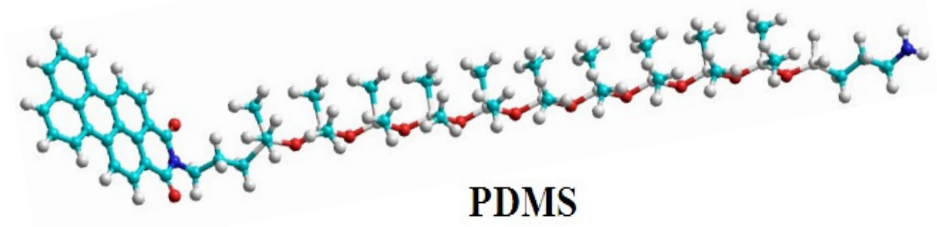

PDI

Propyl amine , Hexane-Water , or Diisopropylamine-Water

\section{Thermo-reversible Gel}
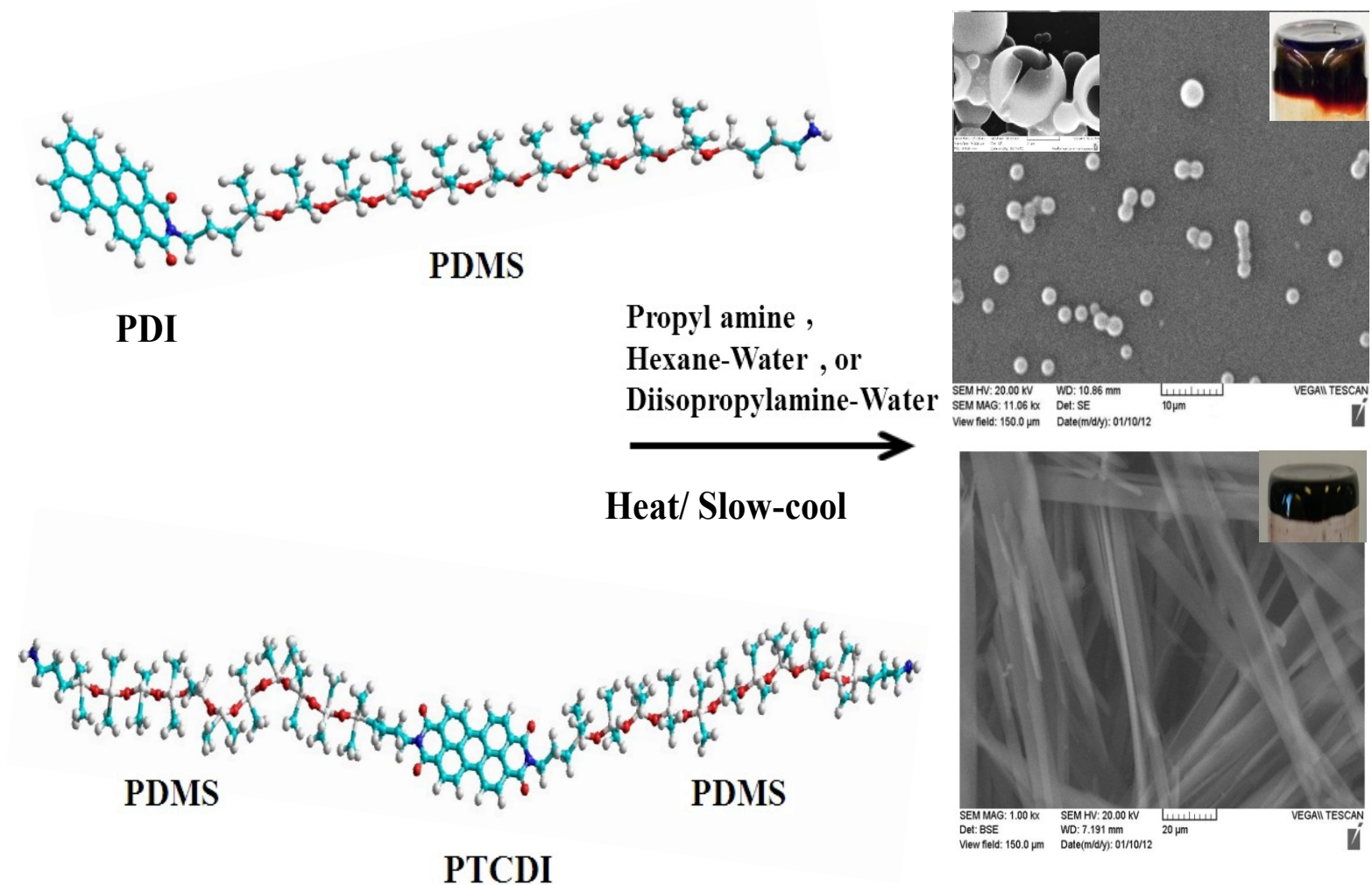

Figure 1.7. Schematic representation of Mono-PDMS and Di-PDMS molecules and SEM images of their respective xerogels made from propyl amine, hexane-water mixture or diisopropylamine-water mixture. Taken from ref 60-62. 

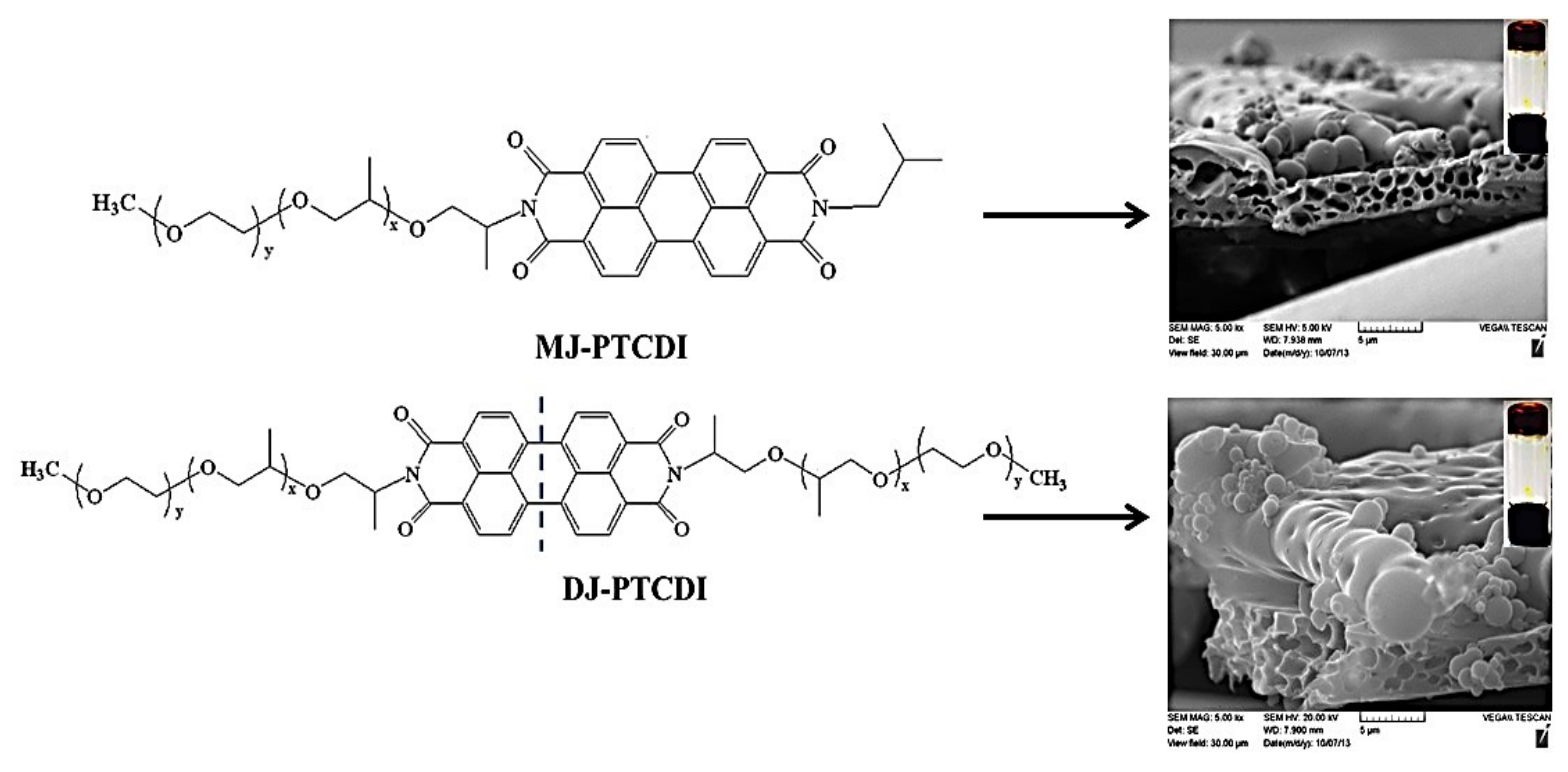

Figure 1.8. Schematic representation of MJ-PTCDI and DJ-PTCDI molecules and SEM images of their respective xerogels showing honeycomb morphology. Taken from ref 63,64 .

In addition to the self-driven factors that influence the formation of a gel such as temperature and concentration, external driving forces have a significant effect on the reversibility of the gels. A gelator's gelling ability in a specified solvent can be evaluated by critical gelation concentration ( $\mathrm{CGC}$ or $\left.\mathrm{C}_{\mathrm{min}}\right)$, which indicates the lowest concentration of gelator molecules required for gelling a liquid at room temperature. At a certain concentration, the gel becomes thermo-reversible, and the phase transition temperature from solution to gel $\left(\mathrm{T}_{\mathrm{gel}}\right)$ can be determined. The methods used to identify these temperatures include differential scanning calorimetry (DSC) and hot-stage microscopy.

In the case of many ureas, amides, amino acid derivatives and carbamates a combination of these hydrogen bonding and van der Waals forces drive gel formation. 
Since not all strongly hydrogen bonding molecules form gels, the van der Waals component of the interactions has strong bearing on the molecules' ability to gel. Based on studies on ureas containing single hydrogen bonding motif and bisureas with two such sites, Feringa et al. concluded that the latter is more susceptible to gelation and that increasing the length of the spacer is also effective. ${ }^{65}$ Here we discuss the gelation behaviour of a set of model compounds for polyurethanes, namely, biscarbamates with two hydrogen bond motifs separated by an alkyl segment, and having terminal alkyl chains of lengths ranging from $\mathrm{C}_{3}$ to $\mathrm{C}_{18}$.

\subsection{Blends}

The synthesis of materials and assembling them into ordered structures to render them functional and operational are crucial aspects of material science. Controlling the outward morphology of such functional materials is of paramount importance. The interplay among the structure, morphology and fabrication processes determines the physical and mechanical properties of the materials. One way to create a whole new material suitable for a specific application is by making a blend. In the industry, polymer blends are common in the production of new polymeric material. There are distinct advantages to form blends. ${ }^{66}$ First, the properties of the blend can be tailored for specific applications by controlling the amount and type of polymer in the mixture. Second, the performance of a high-cost polymer can be extended by blending it with a low-cost material. Finally, the toughness and processability of brittle materials can be improved by adding rubbery or elastomeric polymers. 
The actual fabrication of polymer blends, however, poses significant challenges. The entropy of mixing, which drives small molecules to form miscible mixtures, is negligibly small for high molecular weight polymers. ${ }^{67}$ As a consequence, most polymer pairs are immiscible and the blends phase separate into macroscopic domains. The presence of these phase-separated regions yields a material with poor mechanical properties. To enhance the structural integrity of these materials, copolymer compatibilizers are added to the mixture. ${ }^{67}$ These chains effectively act as high molecular weight surfactants: they localize at the interface between the immiscible polymers, lower the interfacial tension, and disperse the incompatible polymers into smaller domains. Consequently, the degree of adhesion between the phase-separated regions and the mechanical properties of the material are greatly improved.

Small molecules can also form blends. There have been many reported case of molecular-polymer blend hybrids for the use of photovoltaic cells ${ }^{68-70}$ Pure small organic molecular blends are also of interest as they can yield material with similar properties as polymer blends and with a lower cost to production. Contrary to polymers, small organic molecules have a larger entropy of mixing just as long as the individual components are complementary. Such directed associations would be analogous to self-assembly where molecules with multiple hydrogen-bonding sites, with specific donor-acceptor sequence are involved. For example, using DAD: ADA and DAAD: ADDA pairs, linear pseudopolymer structures, and ribbon-like polymers, respectively, have been achieved. ${ }^{71}$ There is also a significant interest currently in the area of organic/inorganic hybrid materials, ${ }^{72}$ organometallics with $\mathrm{M}-\mathrm{H} \cdots \cdot \mathrm{OC}$ type of hydrogen bonding,${ }^{73}$ donor/acceptor complexes, etc., which involve blending/ doping in order to create such hybrid systems. 
Much like polymer blends, it is still a challenge to produce small organic molecule blends that would behave as expected. The crystallinity, spherulitic morphology, and spherulite growth rate are all crucial to the quality of the blend. However, depending on the manner of which the blends were formed, the outcome can be drastic. Here, three different types of molecular blend behaviours will be discussed. They are the self-sorting, the sergeant-soldier, and the majority rules principles.

\subsubsection{Self-Sorting}

Self-sorting is the behaviour of molecules to distinguish between their self and nonself species and, from that, to self-assemble selectively with their corresponding units. ${ }^{74-77}$ This behaviour has been widely observed in biological systems like DNA replication and transcription. ${ }^{78,79}$ In synthetic systems, self-sorting polymers have been observed. For instance, polymers with hydrogen-bonding side chains, such as thymine and cyanuric acid based recognition units were shown to self-assemble with their complementary diamido pyridine and isophthalic wedge moieties. ${ }^{80,81}$

In general, self-sorting events are directed by the same intermolecular forces which govern any molecular recognition process, i.e. hydrogen bonds, metal-ligand interactions, electrostatic interactions, $\pi$ - $\pi$-stacking, and solvophobic effects, and therefore, the factors that determine these recognition events will compromise the fidelity of the self-sorting processes. Molecules or aggregates that show affinity for like species give rise to narcissistic self-sorting (also called self-recognition), whereas the discrimination of equal molecules and the binding between unlike species can be termed social self-sorting or selfdiscrimination (Figure 1.9) ${ }^{82}$ In the reported cases of general self-sorting, there are relevant 
implications of chiral recognition in these systems. ${ }^{83}$

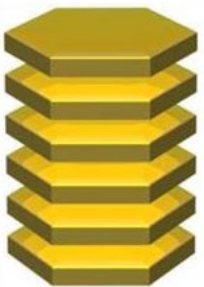

Narcissistic
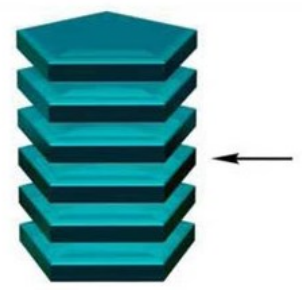

$\sqrt{2}$

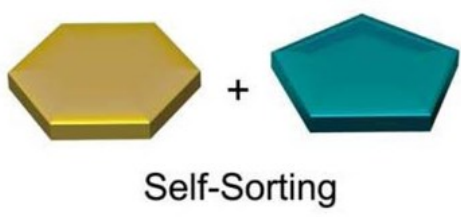

Self-Sorting

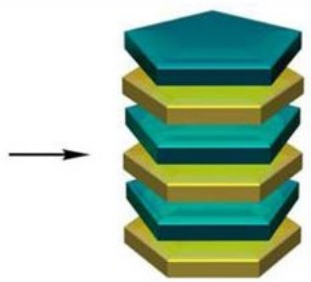

Social

Figure 1.9. Depiction of narcissistic and social self-sorting phenomena in multicomponent mixtures. Taken from ref 82 .

Chiral self-sorting is classified as well into self-recognition, depending on whether an enantiomer recognizes itself or its mirror image leading to the formation of homo- or heterochiral species, respectively. It was first observed with Pasteur's original demonstration of the spontaneous resolution of tartrate salts during crystallization. ${ }^{84}$ The importance of chiral self-sorting is evident, if we take into account the chiral nature of biomolecules such as sugars or amino acids, and organic synthesis as demonstrated in asymmetric catalysis. ${ }^{85,86}$ In the context of covalent polymers, formation of homochiral sequences from a racemic blend of monomeric amino acids by a combination of kinetically determined selection steps has been well observed. ${ }^{87}$

\subsubsection{Sergeant-Soldier Effect}

The Italian school of polymer stereochemistry long ago demonstrated that copolymers prepared from achiral and chiral vinyl monomers show disproportionately high 
optical activities. Although the optical rotatory dispersion of the responsible backbone electronic transitions could not be directly observed, this characteristic was reasonably attributed to induced chiral conformations of the polymer chains. ${ }^{88-90}$ In addition, amplification of chirality is a well-known phenomenon in solutions of stiff helical polymers ${ }^{91}$ as see in Figure 1.10. Pioneering studies by Green and co-workers distinguished the effect that influence the amplification of chirality in poly(isocyanates) and referred to the occurrence as the "sergeant-soldier" principle. ${ }^{88-90}$ The sergeant-soldier principle implies the control of the movements of large numbers of cooperative achiral units (the soldier) by a few chiral units (the sergeant).

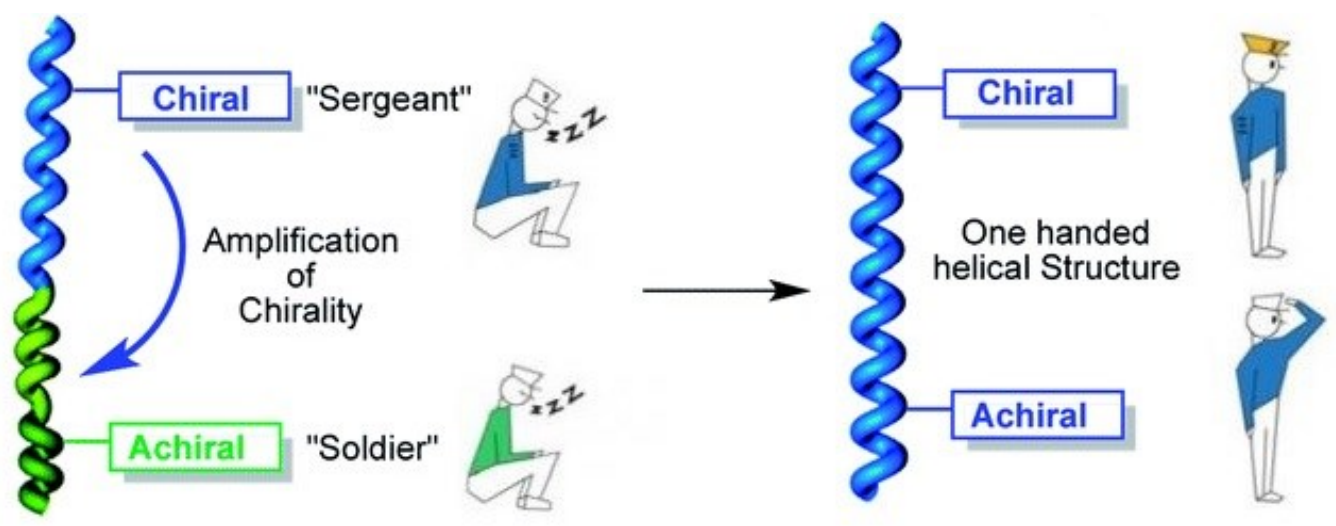

Figure 1.10. Conceptual representation of the sergeant-soldier effect when in presence of a chiral amplification on helical polymers. Image adapted from ref ${ }^{91}$.

Over the past few years, several examples have been presented showing that the sergeant-and-soldier principle is also operational in noncovalently bound, low molecular weight systems. ${ }^{92,93}$ For example, $\mathrm{C}_{3}$-symmetrical disk-shaped compounds forming helical 
columnar stacks as a result of cooperative $\pi-\pi$ stacking and intermolecular H-bonding interactions were found to show strong sergeant-soldier effects both in polar and apolar solvents (Figure 1.11). ${ }^{94}$ However in all of these cases, a chiral molecule was necessary to act as the sergeant. When added to sample of chiral molecules, the non-chiral molecules follow the same morphology (twist, helical, etc) as that of the first during self-assembly. ${ }^{94}$ The chiral molecule acts as a sergeant, and the non-chiral molecule follows it as a soldier. Later on, we will show one example where both molecules are achiral, which involves the biscarbamate molecules.
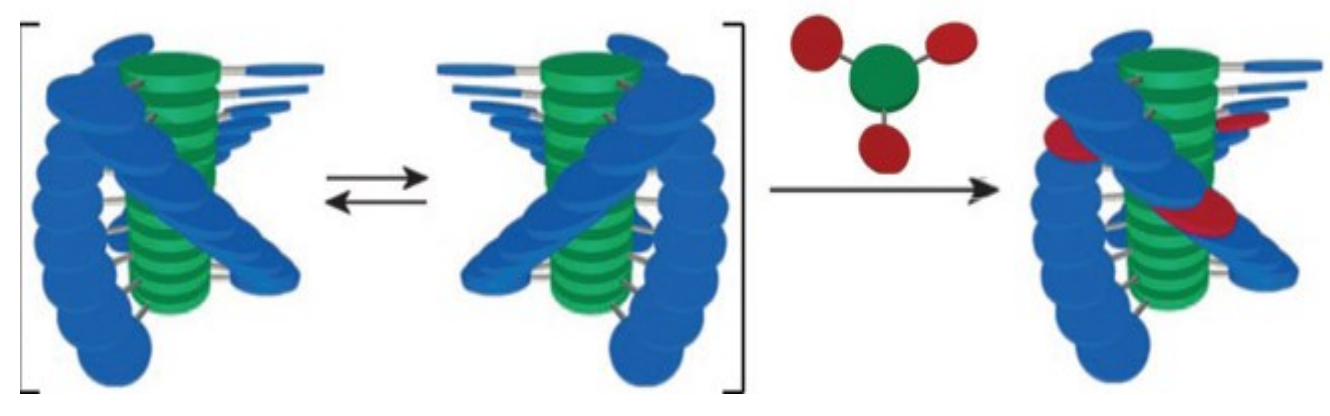

Figure 1.11. A schematic representation of the sergeants-and-soldiers effect determining a preferred handedness of the helical stacks formed by achiral attached disc shaped molecules by doping them with a small amount of chiral molecule. Adapted from ref 94.

\section{$\underline{1.3 .3 \text { Majority Rules }}$}

Amplification of chirality is a well-known phenomenon in solutions of helical copolymers. ${ }^{88,89}$ Of the two classes of chirality amplification, the sergeant-soldier type has been studied most extensively, while the majority rules type is somewhat less well-known. 
The sergeant-soldier principle is characterized by a strongly nonlinear response of the optical properties of the (achiral) polymers to the addition of a small amount of homochiral material, whereas the majority rules principle represents a similar effect for chains consisting of both enantiomeric forms of the chiral monomers, one of which is present in (small) excess. ${ }^{95}$ Both effects occur due to a shift in the balance between left- and righthanded helical bonds, which can be quite large. In other words, the blends exhibiting the majority rules behaviour show physical, optical and morphological properties depending on the component with the higher concentration in the blend. As a good example, majorityrules effects are often found in liquid crystal (LC) phases in comparison to dilute solution (Figure 1.12). ${ }^{93}$ Both types of chirality amplification have recently been studied experimentally, ${ }^{88,90,96-98}$ as well as theoretically, ${ }^{95,97}$ and are found to occur both in covalently bound polymers ${ }^{89,90,92,93,95}$ and in supramolecular (i.e., self-assembled) ones. ${ }^{88,94,98}$ 


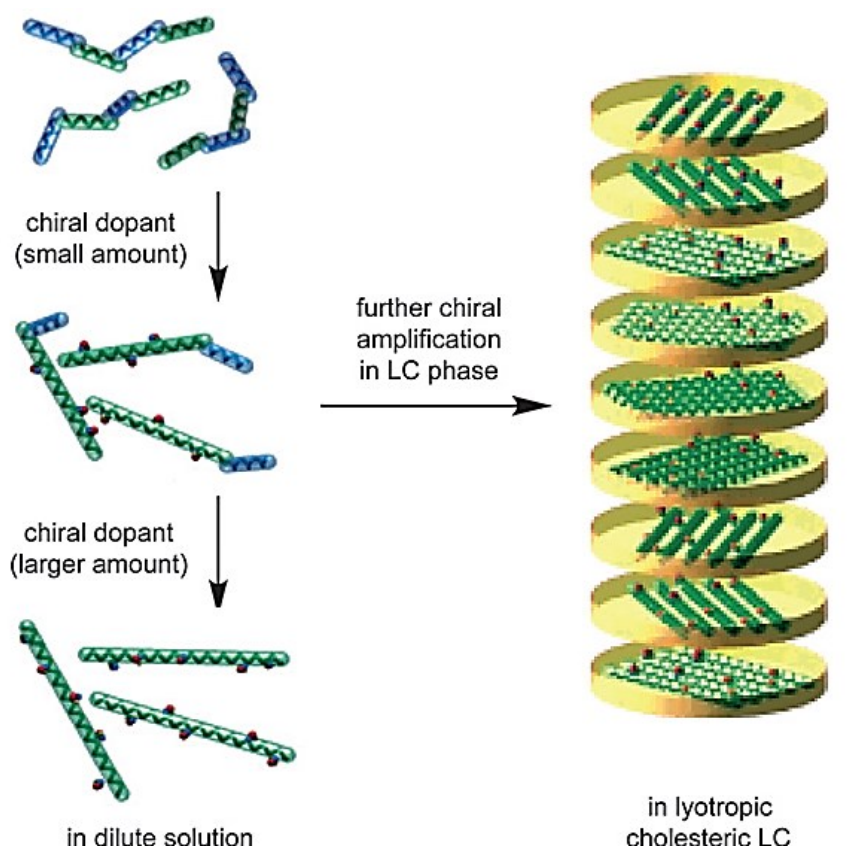

Figure 1.12. A schematic illustration of the induction of one-handed macromolecular helicity in dilute solution and the additional chiral amplification in the LC phase. Adapted from ref ${ }^{93}$.

In this thesis, we intend to show that the self-sorting, the sergeant-soldier and the majority rules effects do not only affect chiral molecules, but they also affect certain types of achiral blends such as biscarbamates. This is indeed interesting as it is the only achiral "amplification" to be reported as of yet.

\subsection{Self-Assembling Biscarbamates}

This thesis deals with the morphological investigations upon the crystallization and the gelation of hydrogen bond mediated self-assembling biscarbamates, which are the 
analogues of the hard segment of polyurethanes.

\subsubsection{Applications of Carbamates and Biscarbamates}

Scientific studies of carbamates date back to early 1900s and continues to the contemporary time because of their potential applications as sequence-specific DNA alkylation agents, ${ }^{99}$ organic intermediates, ${ }^{100}$ photosynthetic inhibitors, ${ }^{101}$ models for alcohol prodrugs, ${ }^{102}$ efficient and nontoxic gene delivery system ${ }^{103}$ and antileukemic agents. ${ }^{104}$ Carbamates and biscarbamates (Figure 1.13) are model compounds for polyurethane. Carbamates have been used in diverse applications since the 1950's such as anti-inflammatory agents ${ }^{105}$ and pesticides. ${ }^{106}$ They have a low melt viscosity of about 8 12 centipoises, a narrow melting temperature $\left(\mathrm{T}_{\mathrm{m}}\right)$ range and rapid crystallization upon quenching from the melt, all of which are ideal for inkjet printing. ${ }^{105}$ The viscosity and $\mathrm{T}_{\mathrm{m}}$ are varied by the choice of length of the alkyl side chains.
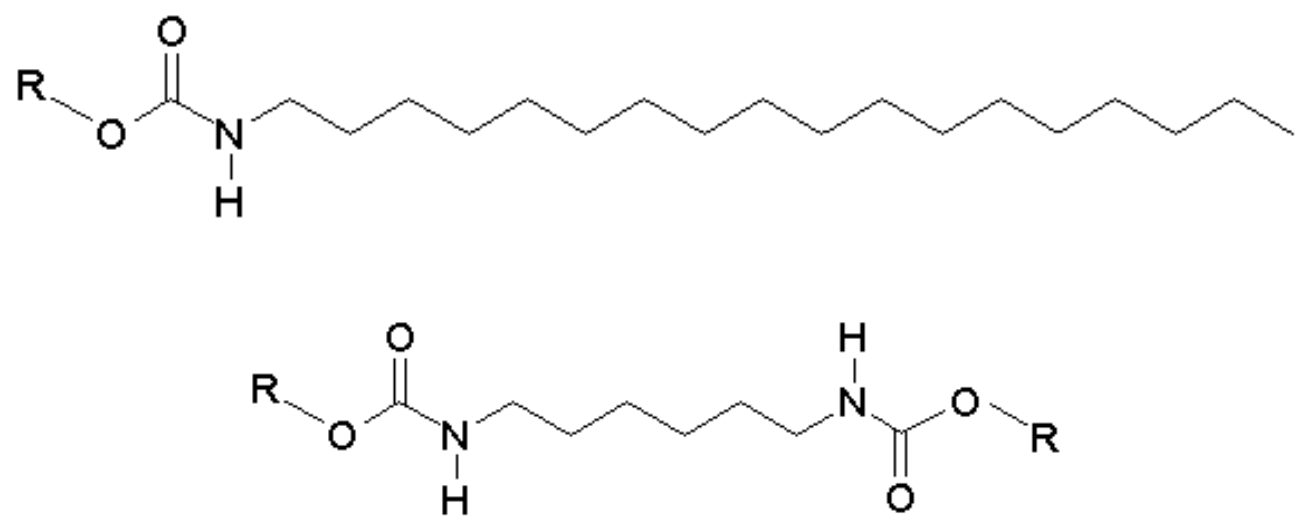

Figure 1.13. Pictorial representation of a $\mathrm{C}_{18}$ carbamate (top) and a $\mathrm{C}_{6}$ spacer biscarbamate (bottom). $\mathrm{R}$ is an alkyl side chain of length varying from $\mathrm{C}_{3}$ to $\mathrm{C}_{18}$. 


\subsubsection{Investigation Using Biscarbamates}

After recent studies on the influence of single versus double hydrogen bonds on carbamates, ${ }^{107}$ it was determined that incorporating multiple hydrogen bonds and varying the alkyl side chain length leads to a more desired morphology in both the solid state ${ }^{107}$ and the gel phase structures. ${ }^{108}$ It was seen that the balance between the hydrogen bonding and van der Waals forces can be optimized both by incorporating multiple hydrogen bonding motifs as well as varying the alkyl side chain length leading to the desired morphology in both the solid state and gel phase structures. The association process in biscarbamates resembles that of biological self-assembly such as protein molecules involving hydrogen bonding as shown in Figure 1.14. Hence, we will be focussing our studies on biscarbamates. For the purposes of this thesis, the nomenclature is based upon only the alkyl side group carbon chain length. The spacer group are fixed at six carbon chain. For convenience, each biscarbamate has been designated as $\mathrm{C}_{\mathrm{x}}$ where $\mathrm{x}$ denotes the number of carbon atoms, derived from the respective alcohols, in the alkyl side chains. The FTIR and ${ }^{1} \mathrm{H}$ NMR spectra for each compound have been placed in Appendix A and B respectively. 


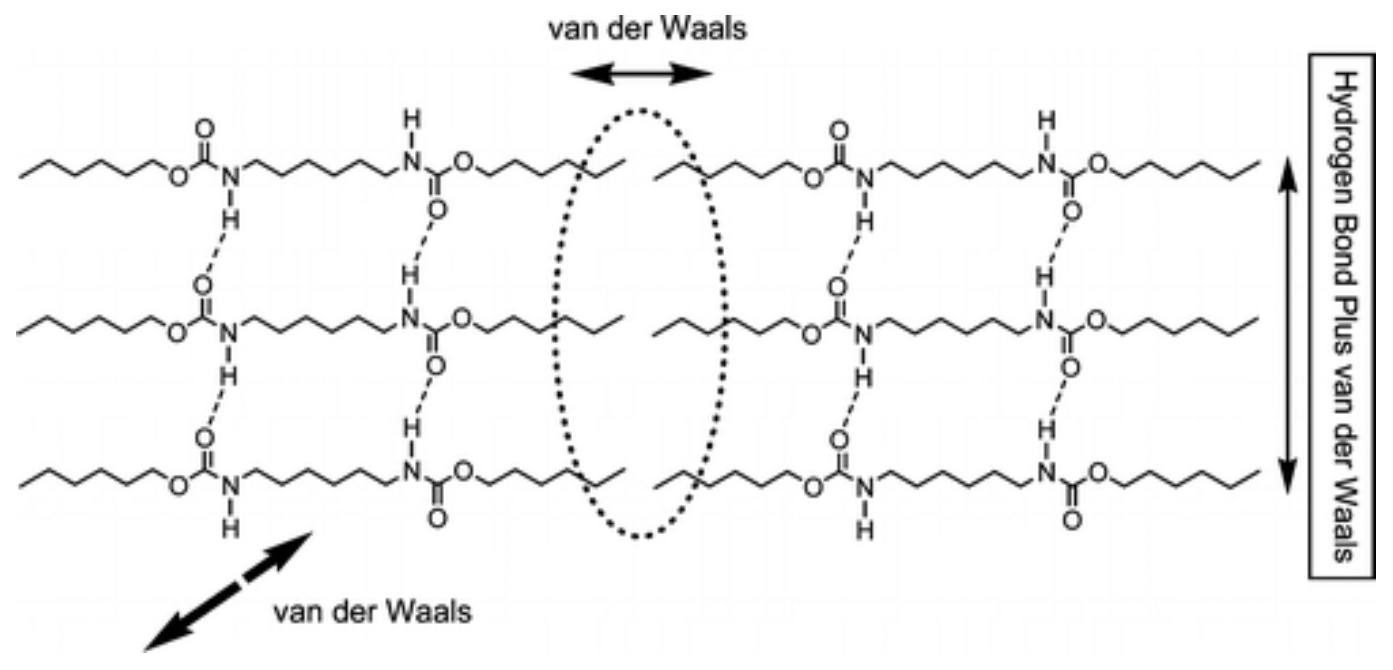

Figure 1.14. Schematic showing the hydrogen bonding and van der Waals interaction in biscarbamates. Taken from ref ${ }^{107}$.

Furthermore, past studies from our group has found many interesting properties of biscarbamates. These molecules with odd versus even number of carbon atoms in the alkyl side chains showed an odd-even alternation in melting temperatures and heats of fusion although the odd-even effect was less pronounced in the latter case. Biscarbamates with odd number of carbon atoms showed higher melting temperatures than the even numbered biscarbamates, which is in contrast to the thermal properties of n-alkanes. ${ }^{109}$ In another instance, the miscibility behaviour of the biscarbamates was studied by melting two molecules with similar spacer but different alkyl side chain length. The result showed that these molecules are capable of hydrogen-bond mediated self-assembly leading to specific association between the same types and does not involve mutual intercalation at the molecular level which implied their immiscibility during blending. ${ }^{110}$ Moreover, biscarbamate gels are first examples of tubular morphology in a non-chiral nor amphiphilic 
system. ${ }^{108}$ These hollow gel fibres were then applied for a novel biscarbamate-based organogel doped with organic dye molecules such as phthalocyanines. ${ }^{111}$ The same molecules form binary gels with polycaprolactone (PCL) in chloroform leading to porous gel networks. $^{112}$

This study aimed at further investigation of biscarbamates with respect to the blends, to the gelation properties of the individual components and of the composites, and to the various methods of gel production.

\subsection{Thesis Objectives}

The overall objective of this thesis was to investigate general behaviour, the resulting morphology and the thermal characteristics of small molecule blends. Biscarbamates in solution blends, gels and gel blends were examined, and trends were established.

The main objectives of this thesis were:

1. To examine the self-sorting behaviours behaviour and methods to overcome the immiscibility of the blends of these biscarbamates with respect to the length and carbon atom parity of the alkyl side chains.

2. To investigate the gelation behaviour and morphology of these biscarbamates in their neat forms as a function of the variation of the alkyl side chain length.

3. To examine the gelation behaviour and morphology of the blends of these 
biscarbamates as a function of alkyl side chain length.

4. To prepare thermally and morphologically similar biscarbamate organogels at lower temperature and lower concentration using microwave heating

5. To explore other solvents viable to produce biscarbamate gels using microwave heating.

This thesis is divided into seven chapters. In Chapter 1, we have given a general introduction to the molecular self-assembly with some discussion on the importance of self-assembling systems. Literature review of self-assembling carbamates and their applications, some basic terminologies related to crystallization, blending behaviours, gelation, and the importance of controlling the morphology also briefly discussed in this chapter.

In this thesis, all the synthesis and experimental methods used will be described in Chapter 2, the study on biscarbamate solution blends will be presented in Chapter 3, the study on odd biscarbamate gels will be presented in Chapter 4, the study on biscarbamate gel blends will be presented in Chapter 5, and the production on microwave biscarbamate gels will be presented in Chapter 6. The thesis will conclude in Chapter 7 with an outlook on these types of biscarbamate gels with a focus on porous gels. 


\subsection{References}

(1) Whitesides, G. M.; Grzybowski, B. Self-Assembly at All Scales. Science. 2002, 295 (5564), 2418-2421.

(2) Philp, D.; Stoddart, J. F. Self-Assembly in Natural and Unnatural Systems. Angew. Chemie Int. Ed. English 1996, 35 (11), 1154-1196.

(3) Lindsey, J. S. Self-Assembly in Synthetic Routes to Molecular Devices. Biological Principles and Chemical Perspectives: A Review. New J. Chem 1991, 15 (2-3), 153179.

(4) Fraenkel-Conrat, H.; Williams, R. C. Reconstitution of Active Tobacco Mosaic Virus from Its Inactive Protein and Nucleic Acid Components. Proc. Natl. Acad. Sci. U. S. A. 1955, 41 (10), 690.

(5) Klug, A. From Macromolecules to Biological Assemblies (nobel Lecture). Angew. Chemie Int. Ed. English 1983, 22 (8), 565-582.

(6) Smith, L. A.; Ma, P. X. Nano-Fibrous Scaffolds for Tissue Engineering. Colloids surfaces B biointerfaces 2004, 39 (3), 125-131.

(7) Ball, P.; Borley, N. R. The Self-Made Tapestry: Pattern Formation in Nature; Oxford University Press Oxford, 1999; Vol. 198.

(8) Zhang, J. Self-Assembled Nanostructures; Springer Science \& Business Media, 2003; Vol. 2.

(9) Crossland, E. J. W.; Ludwigs, S.; Hillmyer, M. A.; Steiner, U. Freestanding 
Nanowire Arrays from Soft-Etch Block Copolymer Templates. Soft Matter 2007, 3 (1), 94-98.

(10) Bates, F. S.; Fredrickson, G. H. Block Copolymer Thermodynamics: Theory and Experiment. Annu. Rev. Phys. Chem. 1990, 41 (1), 525-557.

(11) Bakshi, M. S.; Sachar, S.; Singh, K.; Shaheen, A. Mixed Micelle Behavior of Pluronic L64 and Triton X-100 with Conventional and Dimeric Cationic Surfactants. J. Colloid Interface Sci. 2005, 286 (1), 369-377.

(12) Chen, J. T.; Thomas, E. L.; Ober, C. K.; Mao, G.-P. Self-Assembled Smectic Phases in Rod-Coil Block Copolymers. Science. 1996, 273 (5273), 343-346.

(13) Aida, T.; Meijer, E. W.; Stupp, S. I. Functional Supramolecular Polymers. Science. 2012, 335 (6070), 813-817.

(14) Nuzzo, R. G.; Fusco, F. A.; Allara, D. L. Spontaneously Organized Molecular Assemblies. 3. Preparation and Properties of Solution Adsorbed Monolayers of Organic Disulfides on Gold Surfaces. J. Am. Chem. Soc. 1987, 109 (8), 2358-2368.

(15) Schenning, A. P. H. J.; Jonkheijm, P.; Peeters, E.; Meijer, E. W. Hierarchical Order in Supramolecular Assemblies of Hydrogen-Bonded Oligo (p-Phenylene Vinylene) S. J. Am. Chem. Soc. 2001, 123 (3), 409-416.

(16) Eddaoudi, M.; Kim, J.; Rosi, N.; Vodak, D.; Wachter, J.; O’Keeffe, M.; Yaghi, O. M. Systematic Design of Pore Size and Functionality in Isoreticular MOFs and Their Application in Methane Storage. Science. 2002, 295 (5554), 469-472.

(17) Ajayaghosh, A.; Praveen, V. K.; Vijayakumar, C. Organogels as Scaffolds for 
Excitation Energy Transfer and Light Harvesting. Chem. Soc. Rev. 2008, 37 (1), $109-122$.

(18) Hartgerink, J. D.; Beniash, E.; Stupp, S. I. Self-Assembly and Mineralization of Peptide-Amphiphile Nanofibers. Science. 2001, 294 (5547), 1684-1688.

(19) Hickey, R. J.; Haynes, A. S.; Kikkawa, J. M.; Park, S.-J. Controlling the SelfAssembly Structure of Magnetic Nanoparticles and Amphiphilic BlockCopolymers: From Micelles to Vesicles. J. Am. Chem. Soc. 2011, 133 (5), 15171525.

(20) Meyer, D. E.; Chilkoti, A. Quantification of the Effects of Chain Length and Concentration on the Thermal Behavior of Elastin-like Polypeptides. Biomacromolecules 2004, 5 (3), 846-851.

(21) Meyer, D. E.; Chilkoti, A. Purification of Recombinant Proteins by Fusion with Thermally-Responsive Polypeptides. Nat. Biotechnol. 1999, 17 (11), 1112-1115.

(22) Peer, D.; Karp, J. M.; Hong, S.; Farokhzad, O. C.; Margalit, R.; Langer, R. Nanocarriers as an Emerging Platform for Cancer Therapy. Nat. Nanotechnol. 2007, $2(12), 751-760$.

(23) Zhang, S. Emerging Biological Materials through Molecular Self-Assembly. Biotechnol. Adv. 2002, 20 (5), 321-339.

(24) Dastidar, P. Supramolecular Gelling Agents: Can They Be Designed? Chem. Soc. Rev. 2008, 37 (12), 2699-2715.

(25) Estroff, L. A.; Hamilton, A. D. Water Gelation by Small Organic Molecules. Chem. 
Rev. 2004, 104 (3), 1201-1218.

(26) Terech, P.; Weiss, R. G. Low Molecular Mass Gelators of Organic Liquids and the Properties of Their Gels. Chem. Rev. 1997, 97 (8), 3133-3160.

(27) Sangeetha, N. M.; Maitra, U. Supramolecular Gels: Functions and Uses. Chem. Soc. Rev. 2005, 34 (10), 821-836.

(28) Buerkle, L. E.; Rowan, S. J. Supramolecular Gels Formed from Multi-Component Low Molecular Weight Species. Chem. Soc. Rev. 2012, 41 (18), 6089-6102.

(29) Gels, M. Materials with Self-Assembled Fibrillar Networks, Ed. RG Weiss and P. Terech. Springer, Dordrecht 2006.

(30) Escuder, B.; Miravet, J. F. Functional Molecular Gels; Royal Society of Chemistry, 2013; Vol. 1.

(31) Lloyd, J.; In, D. Colloid Chemistry. Chem. Cat. Co., New York 1926, 767.

(32) Osada, Y.; Ping Gong, J.; Tanaka, Y. Polymer Gels. J. Macromol. Sci. Part C 2004, $44(1), 87-112$.

(33) Guenet, J. Microfibrillar Networks: Polymer Thermoreversible Gels vs Organogels. In Macromolecular Symposia; Wiley Online Library, 2006; Vol. 241, pp 45-50.

(34) Guenet, J.-M. Thermoreversible Gelation of Polymers and Biopolymers; Academic $\operatorname{Pr}, 1992$.

(35) Guenet, J. M.; Wittmann, J. C.; Lotz, B. Thermodynamic Aspects and Morphology of Physical Gels from Isotactic Polystyrene. Macromolecules 1985, 18 (3), 420- 
427.

(36) Sundararajan, P. R.; Tyrer, N.; Bluhm, T. Solvent-Dependent Conformations in Gels of Isotactic Polystyrene. Macromolecules 1982, 15 (2), 286-290.

(37) Atkins, E. D. T.; Isaac, D. H.; Keller, A.; Miyasaka, K. Analysis of Anomalous XRay Diffraction Effects of Isotactic Polystyrene Gels and Its Implications for Chain Conformation and Isomeric Homogeneity. J. Polym. Sci. Polym. Phys. Ed. 1977, 15 (2), 211-226.

(38) Girolamo, M.; Keller, A.; Miyasaka, K.; Overbergh, N. Gelation-Crystallization in Isotactic Polystyrene Solutions and Its Implications to Crystal Morphology, to the Origin and Structure of Gels, and to the Chemical Homogeneity of Polyolefins. $J$. Polym. Sci. Polym. Phys. Ed. 1976, 14 (1), 39-61.

(39) Atkins, E. D. T.; Isaac, D. H.; Keller, A. Conformation of Polystyrene with Special Emphasis to the near All-Trans Extended-Chain Model Relevant in Polystyrene Gels. J. Polym. Sci. Polym. Phys. Ed. 1980, 18 (1), 71-82.

(40) Wellinghoff, S.; Shaw, J.; Baer, E. Polymeric Materials from the Gel State. The Development of Fringed Micelle Structure in a Glass. Macromolecules 1979, 12 (5), 932-939.

(41) Luo, Y.; Kayakabe, S.; Xi, Y.; Bin, Y.; Xu, C.; Matsuo, M. Characteristics of Several Kinds of Polyethylene Gel Estimated by Small-angle Light Scattering under Cross Polarization. J. Polym. Sci. Part B Polym. Phys. 2011, 49 (5), 384-397.

(42) Hikmet, R. M.; Callister, S.; Keller, A. Thermoreversible Gelation of Atactic 
Polystyrene: Phase Transformation and Morphology. In Integration of Fundamental Polymer Science and Technology—2; Springer, 1988; pp 306-316.

(43) de Jong, J. J. D.; Lucas, L. N.; Kellogg, R. M.; van Esch, J. H.; Feringa, B. L. Reversible Optical Transcription of Supramolecular Chirality into Molecular Chirality. Science (80-. ). 2004, 304 (5668), 278-281.

(44) Kato, T. Self-Assembly of Phase-Segregated Liquid Crystal Structures. Science (80.). 2002, 295 (5564), 2414-2418.

(45) van Bommel, K. J. C.; Friggeri, A.; Shinkai, S. Organic Templates for the Generation of Inorganic Materials. Angew. Chemie Int. Ed. 2003, 42 (9), 980-999.

(46) Wynne, A.; Whitefield, M.; Dixon, A. J.; Anderson, S. An Effective, Cosmetically Acceptable, Novel Hydro-Gel Emollient for the Management of Dry Skin Conditions. J. Dermatolog. Treat. 2002, 13 (2), 61-66.

(47) van Esch, J. H.; Feringa, B. L. New Functional Materials Based on Self-Assembling Organogels: From Serendipity towards Design. Angew. Chemie Int. Ed. 2000, 39 (13), 2263-2266.

(48) Brinksma, J.; Feringa, B. L.; Kellogg, R. M.; Vreeker, R.; van Esch, J. Rheology and Thermotropic Properties of Bis-Urea-Based Organogels in Various Primary Alcohols. Langmuir 2000, 16 (24), 9249-9255.

(49) Flory, P. J. Introductory Lecture. Faraday Discuss. Chem. Soc. 1974, 57, 7-18.

(50) Fenniri, H.; Mathivanan, P.; Vidale, K. L.; Sherman, D. M.; Hallenga, K.; Wood, K. V; Stowell, J. G. Helical Rosette Nanotubes: Design, Self-Assembly, and 
Characterization. J. Am. Chem. Soc. 2001, 123 (16), 3854-3855.

(51) Israelachvili, J. N. Intermolecular and Surface Forces: Revised Third Edition; Academic press, 2011.

(52) Liu, X. Y.; Sawant, P. D.; Tan, W. B.; Noor, I. B. M.; Pramesti, C.; Chen, B. H. Creating New Supramolecular Materials by Architecture of Three-Dimensional Nanocrystal Fiber Networks. J. Am. Chem. Soc. 2002, 124 (50), 15055-15063.

(53) Liu, X. Y.; Sawant, P. D. Micro/Nanoengineering of the Self-Organized ThreeDimensional Fibrous Structure of Functional Materials. Angew. Chemie 2002, 114 (19), 3793-3797.

(54) Liu, X. Y.; Sawant, P. D. Mechanism of the Formation of Self-Organized Microstructures in Soft Functional Materials. Adv. Mater. 2002, 14 (6), 421.

(55) Lescanne, M.; Colin, A.; Mondain-Monval, O.; Fages, F.; Pozzo, J.-L. Structural Aspects of the Gelation Process Observed with Low Molecular Mass Organogelators. Langmuir 2003, 19 (6), 2013-2020.

(56) Abdallah, D. J.; Sirchio, S. A.; Weiss, R. G. Hexatriacontane Organogels. The First Determination of the Conformation and Molecular Packing of a Low-MolecularMass Organogelator in Its Gelled State. Langmuir 2000, 16 (20), 7558-7561.

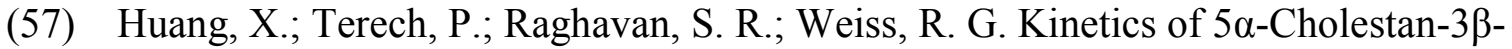
Y1 N-(2-Naphthyl) Carbamate/n-Alkane Organogel Formation and Its Influence on the Fibrillar Networks. J. Am. Chem. Soc. 2005, 127 (12), 4336-4344.

(58) Raghavan, S. R. Distinct Character of Surfactant Gels: A Smooth Progression from 
Micelles to Fibrillar Networks $\dagger$. Langmuir 2009, 25 (15), 8382-8385.

(59) Dahan, E.; Sundararajan, P. R. Polymer Compatibilized Self-Assembling Perylene Derivatives. Eur. Polym. J. 2015, 65, 4-14.

(60) Sundararajan, P. R.; Dahan, E. Thermo-Reversible Physical Gels of Poly (dimethylsiloxane). Polym. Prepr. 2011, 52 (2), 385.

(61) Dahan, E.; Sundararajan, P. R. Thermoreversible Physical Gels of Poly (dimethylsiloxane) without Cross-Links or Functionalization. Langmuir 2013, 29 (27), 8452-8458.

(62) Dahan, E.; Sundararajan, P. R. Thermo-Reversible Gelation of Rod-Coil and CoilRod-Coil Molecules Based on Poly (dimethyl Siloxane) and Perylene Imides and Self-Sorting of the Homologous Pair. Soft Matter 2014, 10 (29), 5337-5349.

(63) Dahan, E.; Sundararajan, P. R. Solvent-Dependent Nanostructures of Gels of a Gemini Surfactant Based on Perylene Diimide Spacer and Oligostyrene Tails. Eur. Polym. J. 2014, 61, 113-123.

(64) Dahan, E. Thermo-Reversible Gelation Of Rod-Coil and Coil-Rod-Coil Molecules Based on Perylene Diimides. Carleton University Ottawa 2013.

(65) van Esch, J.; Kellogg, R. M.; Feringa, B. L. Di-Urea Compounds as Gelators for Organic Solvents. Tetrahedron Lett. 1997, 38 (2), 281-284.

(66) Wool, R.; Sun, X. S. Bio-Based Polymers and Composites; Academic Press, 2011.

(67) Coleman, M. M.; Painter, P. C.; Graf, J. F. Specific Interactions and the Miscibility 
of Polymer Blends; CRC Press, 1995.

(68) Li, G.; Shrotriya, V.; Huang, J.; Yao, Y.; Moriarty, T.; Emery, K.; Yang, Y. HighEfficiency Solution Processable Polymer Photovoltaic Cells by Self-Organization of Polymer Blends. Nat. Mater. 2005, 4 (11), 864-868.

(69) Yu, G.; Gao, J.; Hummelen, J. C.; Wudl, F.; Heeger, A. J. Polymer Photovoltaic Cells: Enhanced Efficiencies via a Network of Internal Donor-Acceptor Heterojunctions. Sci. Pap. Ed. 1995, 270 (5243), 1789-1790.

(70) Dittmer, J. J.; Marseglia, E. A.; Friend, R. H. Electron Trapping in Dye/polymer Blend Photovoltaic Cells. Adv. Mater. 2000, 12 (17), 1270-1274.

(71) Ikeda, M.; Nobori, T.; Schmutz, M.; Lehn, J. Hierarchical Self-Assembly of a BowShaped Molecule Bearing Self-Complementary Hydrogen Bonding Sites into Extended Supramolecular Assemblies. Chem. Eur. J. 2005, 11 (2), 662-668.

(72) Laeri, F.; Schüth, F.; Simon, U.; Wark, M. Host-Guest-Systems Based on Nanoporous Crystals; John Wiley \& Sons, 2006.

(73) Braga, D.; Grepioni, F.; Desiraju, G. R. Hydrogen Bonding in Organometallic Crystals-a Survey. J. Organomet. Chem. 1997, 548 (1), 33-43.

(74) Lehn, J.-M. Toward Self-Organization and Complex Matter. Science. 2002, 295 (5564), 2400-2403.

(75) Lehn, J.-M. Programmed Chemical Systems: Multiple Subprograms and Multiple Processing/Expression of Molecular Information. Chem. Eur. J. 2000, 6 (12), 2097- 
2102.

(76) Saur, I.; Scopelliti, R.; Severin, K. Utilization of Self-Sorting Processes To Generate Dynamic Combinatorial Libraries with New Network Topologies. Chem. Eur. J. 2006, 12 (4), 1058-1066.

(77) Wu, A.; Isaacs, L. Self-Sorting: The Exception or the Rule? J. Am. Chem. Soc. 2003, $125(16), 4831-4835$.

(78) Nelson, D. L.; Lehninger, A. L.; Cox, M. M. Lehninger Principles of Biochemistry; Lehninger Principles of Biochemistry; W. H. Freeman, 2008.

(79) Watson, J. D.; Crick, F. H. C. Molecular Structure of Nucleic Acids. Nature 1953, 171 (4356), 737-738.

(80) Berl, V.; Schmutz, M.; Krische, M. J.; Khoury, R. G.; Lehn, J.-M. Supramolecular Polymers Generated from Heterocomplementary Monomers Linked through Multiple Hydrogen-Bonding Arrays Formation, Characterization, and Properties. Chem. A Eur. J. 2002, 8 (5), 1227-1244.

(81) Lehn, J. M. Supramolecular Chemistry: Concepts and Perspectives; George Fisher Baker lectures in chemistry; Wiley, 1995.

(82) Rest, C.; Mayoral, M. J.; Fernández, G. Aqueous Self-Sorting in Extended Supramolecular Aggregates. Int. J. Mol. Sci. 2013, 14 (1), 1541-1565.

(83) Pijper, D.; Feringa, B. L. Control of Dynamic Helicity at the Macro-and 
Supramolecular Level. Soft Matter 2008, 4 (7), 1349-1372.

(84) Pasteur, L. "Recherches Sur Les Relations Qui Peuvent Exister Entre La Forme Crystalline, La Composition Chimique et Le Sens de La Polarisation Rotatoire. Ann. Chim. Phys. 1848, 24, 442-459.

(85) Lacour, J.; Linder, D. A Counterion Strategy. Science (80-. ). 2007, 317 (5837), $462-463$.

(86) Mohr, J. T.; Krout, M. R.; Stoltz, B. M. Natural Products as Inspiration for the Development of Asymmetric Catalysis. Nature 2008, 455 (7211), 323-332.

(87) Burd, C.; Weck, M. Self-Sorting in Polymers. Macromolecules 2005, 38 (17), 72257230.

(88) Green, M. M.; Park, J.; Sato, T.; Teramoto, A.; Lifson, S.; Selinger, R. L. B.; Selinger, J. V. The Macromolecular Route to Chiral Amplification. Angew. Chemie Int. Ed. 1999, 38 (21), 3138-3154.

(89) Green, M. M.; Peterson, N. C.; Sato, T.; Teramoto, A. A Helical Polymer with a Cooperative Response to Chiral Information. Science (80-. ). 1995, 268 (5219), 1860.

(90) Green, M. M.; Reidy, M. P.; Johnson, R. D.; Darling, G.; O’Leary, D. J.; Willson, G. Macromolecular Stereochemistry: The out-of-Proportion Influence of Optically Active Comonomers on the Conformational Characteristics of Polyisocyanates. The Sergeants and Soldiers Experiment. J. Am. Chem. Soc. 1989, 111 (16), 6452-6454.

(91) Bergueiro, J.; Freire, F.; Wendler, E. P.; Seco, J. M.; Quiñoá, E.; Riguera, R. The 
ON/OFF Switching by Metal Ions of the "Sergeants and Soldiers" Chiral Amplification Effect on Helical Poly (phenylacetylene) S. Chem. Sci. 2014, 5 (6), $2170-2176$.

(92) Brunsveld, L.; Vekemans, J.; Hirschberg, J.; Sijbesma, R. P.; Meijer, E. W. Hierarchical Formation of Helical Supramolecular Polymers via Stacking of Hydrogen-Bonded Pairs in Water. Proc. Natl. Acad. Sci. 2002, 99 (8), 4977-4982.

(93) Maeda, K.; Takeyama, Y.; Sakajiri, K.; Yashima, E. Nonracemic Dopant-Mediated Hierarchical Amplification of Macromolecular Helicity in a Charged Polyacetylene Leading to a Cholesteric Liquid Crystal in Water. J. Am. Chem. Soc. 2004, 126 (50), $16284-16285$.

(94) Palmans, A. R. A.; Meijer, E. emsp14W. Amplification of Chirality in Dynamic Supramolecular Aggregates. Angew. Chemie Int. Ed. 2007, 46 (47), 8948-8968.

(95) Green, M. M.; Garetz, B. A.; Munoz, B.; Chang, H.; Hoke, S.; Cooks, R. G. Majority Rules in the Copolymerization of Mirror Image Isomers. J. Am. Chem. Soc. 1995, $117(14), 4181-4182$.

(96) Berova, N.; Nakanishi, K. Circular Dichroism: Principles and Applications; John Wiley \& Sons, 2000.

(97) Brunsveld, L.; Lohmeijer, B. G. G.; Vekemans, J. A. J. M.; Meijer, E. W. Chirality Amplification in Dynamic Helical Columns in Water. Chem. Commun. 2000, No. $23,2305-2306$.

(98) Brunsfeld, L.; Lohmeijer, B. G. G.; Vekemans, J. A. J. M.; Meijer, E. W. 
Amplification of Chirality in Helical Supramolecular Columns. J. Incl. Phenom. Macrocycl. Chem. 2001, 41 (1-4), 61-64.

(99) Saka, K.; Noda, K. Jpn. Kokai Tokkyo Koho JP 62179 584, 1987. In Chem. Abstr; 1987; Vol. 108, p 39820r.

(100) Gaylord, N. G. Carbamates III. Reactions of "Acetylene Bis-Carbamates" (1). J. Org. Chem. 1955, 20 (4), 546-548.

(101) Salvucci, M. E.; Crafts-Brandner, S. J. Inhibition of Photosynthesis by Heat Stress: The Activation State of Rubisco as a Limiting Factor in Photosynthesis. Physiol. Plant. 2004, 120 (2), 179-186.

(102) Papot, S.; Bachmann, C.; Combaud, D.; Gesson, J.-P. Study of Biscarbamates Derived from 2-Aminobenzylamines as Models for Alcohol Prodrugs. Tetrahedron 1999, 55 (15), 4699-4708.

(103) Fan, H.; Yang, K.; Boye, D. M.; Sigmon, T.; Malloy, K. J.; Xu, H.; López, G. P.; Brinker, C. J. Self-Assembly of Ordered, Robust, Three-Dimensional Gold Nanocrystal/silica Arrays. Science. 2004, 304 (5670), 567-571.

(104) Anderson, W. K.; Mach, R. H. Synthesis, Chemical Reactivity, and Antileukemic Activity of 5-Substituted 6, 7-Bis (hydroxymethyl) Pyrrolo [1, 2-C] Thiazole Biscarbamates and the Corresponding Sulfoxides and Sulfones. J. Med. Chem. 1987, 30 (11), 2109-2115.

(105) Tanaka, K.; Kano, Y.; Yoshida, K. Jpn. Kokai Tokkyo Koho JP 63248 894, 1988. 
In Chem. Abstr; 1988; Vol. 110, p 79137w.

(106) Steichele, K. Belg. Patent 882,922, 1980. In Chem. Abstr; 1980; Vol. 94, p 66768d.

(107) Khanna, S.; Moniruzzaman, M.; Sundararajan, P. R. Influence of Single versus Double Hydrogen-Bonding Motif on the Crystallization and Morphology of SelfAssembling Carbamates with Alkyl Side Chains: Model System for Polyurethanes. J. Phys. Chem. B 2006, 110 (31), 15251-15260.

(108) Khanna, S.; Khan, M. K.; Sundararajan, P. Influence of Double Hydrogen Bonds and Alkyl Chains on the Gelation of Nonchiral Polyurethane Model Compounds: Sheets, Eaves Trough, Tubes and Oriented Fibers. Langmuir 2009, 25 (22), 1318313193.

(109) Khan, M. K.; Sundararajan, P. R. Effects of Carbon Atom Parity and Alkyl Side Chain Length on the Crystallization and Morphology of Biscarbamates, A Set of Model Compounds for Polyurethanes. J. Phys. Chem. B 2011, 115 (27), 8696-8706.

(110) Khan, M. K.; Sundararajan, P. R. Molecular Selectivity and Immiscibility during the Crystallization of Mixtures of a Set of Homologous Self-Assembling Molecules. $J$. Phys. Chem. B 2008, 112 (14), 4223-4232.

(111) Khan, M. K.; Sundararajan, P. Encapsulation of Dye Molecules and Nanoparticles in Hollow Organogel Fibers of a Nonchiral Polyurethane Model Compound. Chem. Eur. J. 2011, 17 (4), 1184-1192.

(112) Khan, M. K.; Chan, A.; Sundararajan, P. R. Two Component Gels of Immiscible Blends of Biscarbamates (polyurethane Model Compounds) and Poly ( $\varepsilon$ - 
Caprolactone). Soft Matter 2012, 8 (39), 10149-10157. 
CHAPTER 2

Methods 


\section{CHAPTER II: METHODS}

\subsection{Introduction}

This chapter describes the synthesis, purification and characterization procedures of the materials used in this thesis. The chemical structures, physical properties and general sample preparation procedures of the materials are included. Any special sample preparation and characterization procedure is described separately in the appropriate sections of this thesis. This chapter also describes the techniques used to study the morphological and thermal behaviour of different members of the biscarbamates class and

of their blends. Techniques include differential scanning calorimetry (DSC), X-ray diffraction (XRD), hot-stage optical microscopy (OM) and scanning electron microscopy (SEM).

\subsection{Biscarbamate Sample Preparations}

\subsubsection{Biscarbamate Synthesis}

The synthesis of biscarbamates used in this study was described by Goodbrand et al. ${ }^{1}$ This involves reacting 1,6 -diisocyanatohexane $(0.1 \mathrm{~mol})$ with various alcohols $(0.2$ mol) ranging from 1-propanol to 1-octadecanol (Scheme 1) separately in the presence of the catalyst 1,4-Diazabicyclo [2,2,2] octane (DABCO). The reaction was carried out in toluene $(200 \mathrm{~mL})$, at $85-90{ }^{\circ} \mathrm{C}$ for 6 hours except for 1 -octadecanol (8-9 hours). All the reagents were purchased from Sigma-Aldrich Inc. and were used as received. 


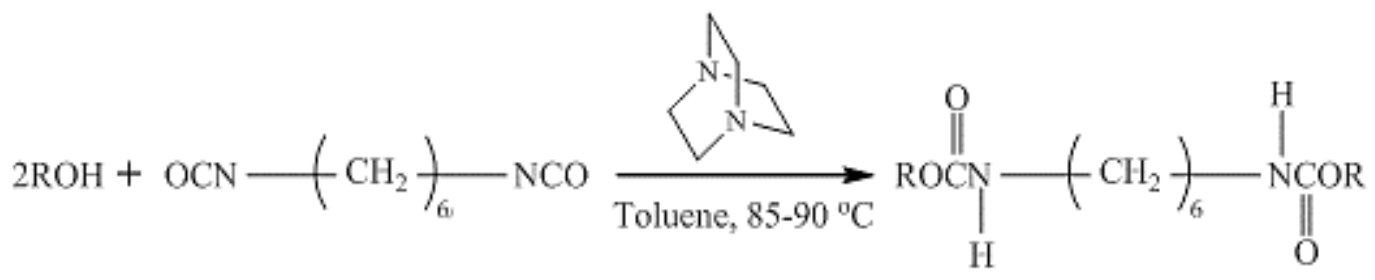

Scheme 2.1. Scheme for synthesis of the biscarbamates from respective diisocyanates and alcohols.

For example, 1,6-hexanediyl-bis-didodecyl carbamate $\left(\mathrm{C}_{12}\right.$ biscarbamate $)$ was prepared by taking $16.82 \mathrm{~g}(0.1 \mathrm{~mol})$ of 1,6-diisocyanato hexane in a $500 \mathrm{~mL}$ round bottom flask equipped with a reflux condenser and a nitrogen purge. Subsequently $37.27 \mathrm{~g}(0.2$ mol) of dodecanol along with $200 \mathrm{~mL}$ of toluene and $20 \mathrm{mg}$ 1,4-diazabicyclo [2,2,2] octane were added to the same flask and the mixture was refluxed for 6 hours. The solution was cooled and $\mathrm{C}_{12}$ biscarbamate was obtained after filtration. Products of the reactions were isolated by suction filtration. These products were purified by recrystallization in toluene at $60{ }^{\circ} \mathrm{C}$. The average yield of the product is about $90 \%$. The recrystallization was repeated twice in order to remove any impurity or unreacted reagent (1,6-diisocyanato hexane and respective alcohols). Infrared, ${ }^{1} \mathrm{H}$ NMR spectroscopy and differential scanning calorimetry were used to confirm the reaction products and to assess their purity. As the resulting spectra showed clean peaks and the resulting endotherm showed a single sharp narrow peak, we can claim that the final product has a high purity $(\sim 95 \%)$.

The names of the synthesized compounds are listed in Table 2.1, and for convenience each of them has been designated as $\mathrm{C}_{x}$ where $x$ denotes the number of carbon atoms, derived from the respective alcohols, in the alkyl side chains. All of the compounds 
contain six (6) carbon atoms in the spacer group.

Table 2.1. Biscarbamates $\left(C_{x}\right)$ synthesized from reaction of an appropriate alcohol with 1,6-diisocyanato hexane.

\begin{tabular}{|c|c|c|c|}
\hline \multicolumn{4}{|c|}{$\mathrm{C}_{x}$ Biscarbamate } \\
\hline ID & parent alcohol & molecular formula & $T_{\mathrm{m}},{ }^{\circ} \mathrm{C}$ \\
\hline $\mathrm{C}_{3}$ & 1-propanol & $\mathrm{H}_{7} \mathrm{C}_{3} \mathrm{OOCHN}\left(\mathrm{CH}_{2}\right)_{6} \mathrm{NHCOOC}_{3} \mathrm{H}_{7}$ & 101.9 \\
\hline $\mathrm{C}_{4}$ & 1-butanol & $\mathrm{H}_{9} \mathrm{C}_{4} \mathrm{OOCHN}\left(\mathrm{CH}_{2}\right)_{6} \mathrm{NHCOOC}_{4} \mathrm{H}_{9}$ & 91.3 \\
\hline $\mathrm{C}_{5}$ & 1-pentanol & $\mathrm{H}_{11} \mathrm{C}_{5} \mathrm{OOCHN}\left(\mathrm{CH}_{2}\right)_{6} \mathrm{NHCOOC}_{5} \mathrm{H}_{11}$ & 103.6 \\
\hline $\mathrm{C}_{6}$ & 1-hexanol & $\mathrm{H}_{13} \mathrm{C}_{6} \mathrm{OOCHN}\left(\mathrm{CH}_{2}\right)_{6} \mathrm{NHCOOC}_{6} \mathrm{H}_{13}$ & 97.1 \\
\hline $\mathrm{C}_{7}$ & 1-heptanol & $\mathrm{H}_{15} \mathrm{C}_{7} \mathrm{OOCHN}\left(\mathrm{CH}_{2}\right)_{6} \mathrm{NHCOOC}_{7} \mathrm{H}_{15}$ & 108.9 \\
\hline $\mathrm{C}_{8}$ & 1-octanol & $\mathrm{H}_{17} \mathrm{C}_{8} \mathrm{OOCHN}\left(\mathrm{CH}_{2}\right)_{6} \mathrm{NHCOOC}_{8} \mathrm{H}_{17}$ & 106.6 \\
\hline $\mathrm{C}_{9}$ & 1-nonanol & $\mathrm{H}_{19} \mathrm{C}_{9} \mathrm{OOCHN}\left(\mathrm{CH}_{2}\right)_{6} \mathrm{NHCOOC}_{9} \mathrm{H}_{19}$ & 113.5 \\
\hline $\mathrm{C}_{10}$ & 1-decanol & $\mathrm{H}_{21} \mathrm{C}_{10} \mathrm{OOCHN}\left(\mathrm{CH}_{2}\right)_{6} \mathrm{NHCOOC}_{10} \mathrm{H}_{21}$ & 112.8 \\
\hline $\mathrm{C}_{11}$ & 1-undecanol & $\mathrm{H}_{23} \mathrm{C}_{11} \mathrm{OOCHN}\left(\mathrm{CH}_{2}\right)_{6} \mathrm{NHCOOC}_{11} \mathrm{H}_{23}$ & 117.9 \\
\hline $\mathrm{C}_{12}$ & 1-dodecanol & $\mathrm{H}_{25} \mathrm{C}_{12} \mathrm{OOCHN}\left(\mathrm{CH}_{2}\right)_{6} \mathrm{NHCOOC}_{12} \mathrm{H}_{25}$ & 114.9 \\
\hline $\mathrm{C}_{13}$ & 1-tridecanol & $\mathrm{H}_{27} \mathrm{C}_{13} \mathrm{OOCHN}\left(\mathrm{CH}_{2}\right)_{6} \mathrm{NHCOOC}_{13} \mathrm{H}_{27}$ & 121.5 \\
\hline $\mathrm{C}_{14}$ & 1-tetradecanol & $\mathrm{H}_{29} \mathrm{C}_{14} \mathrm{OOCHN}\left(\mathrm{CH}_{2}\right)_{6} \mathrm{NHCOOC}_{14} \mathrm{H}_{29}$ & 116.7 \\
\hline $\mathrm{C}_{15}$ & 1-pentadecanol & $\mathrm{H}_{31} \mathrm{C}_{15} \mathrm{OOCHN}\left(\mathrm{CH}_{2}\right)_{6} \mathrm{NHCOOC}_{15} \mathrm{H}_{31}$ & 123.2 \\
\hline $\mathrm{C}_{16}$ & 1-hexadecanol & $\mathrm{H}_{33} \mathrm{C}_{16} \mathrm{OOCHN}\left(\mathrm{CH}_{2}\right)_{6} \mathrm{NHCOOC}_{16} \mathrm{H}_{33}$ & 117.8 \\
\hline $\mathrm{C}_{18}$ & 1-octadecanol & $\mathrm{H}_{37} \mathrm{C}_{18} \mathrm{OOCHN}\left(\mathrm{CH}_{2}\right)_{6} \mathrm{NHCOOC}_{18} \mathrm{H}_{37}$ & 120.4 \\
\hline
\end{tabular}




\subsubsection{Biscarbamate Blends Preparation}

Melt mixing was performed by heating pre-weighed mixtures of biscarbamates in a $5 \mathrm{~mL}$ vial at a temperature of $20^{\circ} \mathrm{C}$ above that of the higher melting component with continuous stirring. For example, a 50:50 mixture of $\mathrm{C}_{4}$ and $\mathrm{C}_{18}$ would be prepared by placing the vial in an oil bath at $140{ }^{\circ} \mathrm{C}$. After 30 minutes of heating, the melt sample in the vial would be slowly cooled to room temperature by simply turning off the hot-plate or cooled by placing it in an ice bath. These techniques are knows as slow-cooling and quenching respectively. Since the melt viscosity of these samples are very low, the samples in the blends are expected to be mixed well. Both blend samples have been shown to have the similar thermodynamic behaviour; ${ }^{2}$ hence, for the purposes of this paper, only results from the slow-cooling method will be presented.

Solvent blends were achieved by dissolving $0.1 \mathrm{~g}$ of the biscarbamate mixtures in $3 \mathrm{~mL}$ of chloroform in a sealed Pyrex vial at $70-80^{\circ} \mathrm{C}$ with continuous stirring. The blends were precipitated out by cooling the sample to room temperature at a rate of $5^{\circ} \mathrm{C}$ per minute and then placed in the fridge. The solvent was evaporated out while the precipitated solution remained cold. The blends were then left to dry at room temperature for a day, and then kept under vacuum for another day to form the dry solvent-based blends.

Three types of binary blends were prepared: biscarbamates with very different, somewhat similar and very close side chain lengths. In correspondence to a previous study, ${ }^{2}$ the binary blends are $\mathrm{C}_{4} / \mathrm{C}_{18}, \mathrm{C}_{8} / \mathrm{C}_{12}, \mathrm{C}_{16} / \mathrm{C}_{18}$ biscarbamates. We mainly looked at even biscarbamate blends, but even-odd biscarbamate and even-odd-even trinary blends are also briefly discussed. 


\subsubsection{Biscarbamate Gels Using Conventional Heating}

As with our previous work, ${ }^{3-5}$ benzonitrile was used as the primarily solvent. Gels of concentration $0.08 \mathrm{M}$ were prepared in capped vials to avoid evaporation of the solvent. Gels were also prepared with DMSO, 1-octanol, xylene, toluene, and dodecane to study the effect of the solvent type. ${ }^{4}$ Conventional hot plate and a microwave source were used for preparation of the gels.

\subsubsection{Conventional Heating}

For the gels prepared using the hot plate, weighed amounts of biscarbamates of different alkyl side chain lengths were dissolved in requisite amount of solvent in a Pyrex vial in an oil bath at around $80-100{ }^{\circ} \mathrm{C}$, which is about $20{ }^{\circ} \mathrm{C}$ above the dissolution temperature, resulting in clear solutions. This would ensure that the solution would be free of any remaining nuclei which promote gelation through a self-seeding process. Unless stated otherwise, the concentration of biscarbamates to solvent is $0.08 \mathrm{M}$, which is the minimum critical gelation concentration for the shortest biscarbamate able to form a gel $\left(\mathrm{C}_{6}\right)$. Gels were made by slowly cooling to room temperature by turning off the hot plate. Tube inversion was used as a checking method for the gelation. The norm that is usually used is that when the vials were turned upside down and if the solute/solvent were not visually separated and did not flow they were considered as gels.

\subsubsection{Microwave Heating}

For the gels prepared using the microwave system, weighed amounts of biscarbamates and the requisite amount of solvent were placed in a $20 \mathrm{~mL}$ Pyrex vial. The vial was then placed in a porcelain vessel into an ETHOS PRO microwave digestive system 
running with the Milestone Digestion operating system (Figure 2.1). The samples were exposed to a maximum power of $1000 \mathrm{~W}$ of radiation as the software automatically delivers the minimum power required to follow the defined temperature profiles. The chamber containing the samples was programmed to set temperatures ranging from 40 to $160{ }^{\circ} \mathrm{C}$ in 10 minutes and to keep that temperature for 30 minutes to two hours. The temperature of the chamber was measured using a temperature probe (ATC-400) inserted inside a reference vessel (Figure 2.1). This allows for the continuous monitoring of the thermal conditions and adjusting the microwave parameters to achieve the desired temperature profile inside all the vessels. ${ }^{6}$ As the concentration and the solvents changed, the set temperature was modified to ascertain that the fully dissolved solutions were obtained prior to lowering the temperature for gelation. Due to visual limitations of the instrument (Figure 2.1), we were not able to determine the dissolution temperature visually. At the end of the entire heating and cooling process, the vials that do produce a uniform gel were considered to have an adequate dissolution temperature, while the samples which show precipitation or partial dissolution are determined to have insufficient heating. The radiation was then terminated and the samples were left to cool to room temperature at a rate of $1{ }^{\circ} \mathrm{C} /$ minute for an hour. Once again, the vials were turned upside down to check for gelation. All the gels were opaque. These gels were dried at room temperature for a day and then kept under vacuum for a few days to form xerogels. There were no signs of degradation or chemical reactions of the biscarbamate molecules when using microwave heating. 


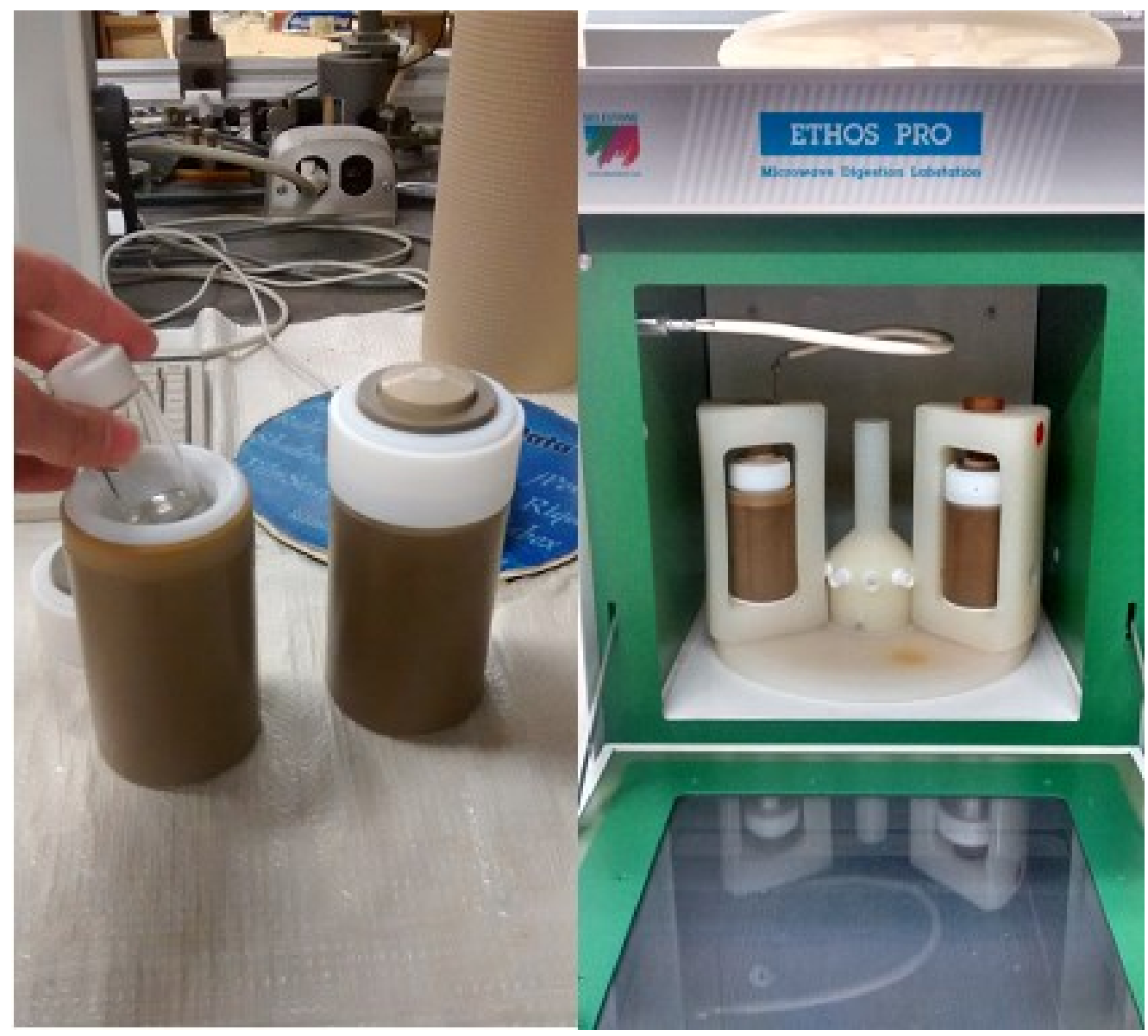

Figure 2.1. (Left) Image of an open ceramic cell where a sample vial is inserted, and a closed ceramic cell. (Right) Image of the microwave digestion apparatus with the reference cell connected to the instrument, and a sample cell.

\subsection{Characterization of the Biscarbamates}

\subsubsection{FTIR Spectra of the Biscarbamates}

Infrared (IR) spectroscopy is one of the most often used spectroscopic tools for the study of characterization of materials. It can be used for both qualitative (fingerprinting of compounds; recognition of specific chemical bonds, or groups; gross structural features $)^{7,8}$ 
and quantitative (measurements of reaction rates, determination of thermodynamic data, process and control) analysis. ${ }^{9-11}$ The basis of IR experiments is to pass infrared radiation through a thin sample and measure which energies of the applied infrared radiation are transmitted by the sample. The characteristic band parameters measured in IR spectroscopy are frequency (energy), intensity, band shape (environments of bonds), and the polarization of various modes (the transition-moment direction in the molecular framework). ${ }^{12-15}$ The position of an infrared absorption band is specified in frequency units by its wavenumber measured in reciprocal centimeter $\left(\mathrm{cm}^{-1}\right)$, or by wavelength measured in nanometer $(\mathrm{nm})$.

Absorption of infrared radiation causes transitions between vibrational energy levels of a molecule, which are also accompanied by several rotational motions. Vibrational energy corresponds to the vibrations of atoms about the mean centre of their chemical bonds, whereas rotational energy changes occur due to tumbling motion of a molecule. ${ }^{712-15}$ In order to absorb IR radiation, a molecule must undergo a net change in dipole moment as a consequence of its vibration. Homonuclear diatomic molecules do not absorb IR radiation. On the other hand, complex mixtures give rise to very crowded spectra in which assigning of bands to specific functional groups is very difficult. Each vibration is unique to a particular bond stretch. ${ }^{7,12-15}$ IR spectra are the often-used technique to study hydrogen bonding. For example, if an $\mathrm{N}-\mathrm{H} \ldots \mathrm{O}=\mathrm{C}$ hydrogen bond is present in a system, the frequencies corresponding to these groups would show a shift from the non-hydrogen bonded frequency. The magnitude of the shifts could be used to calculate the hydrogen bond distances. ${ }^{2}$

FTIR spectra of the biscarbamate samples were taken at the Department of Chemistry at Carleton University. The samples were prepared for IR spectroscopy by 
mixing $2 \mathrm{mg}$ of the biscarbamates with dried $\mathrm{KBr}$ and were ground into fine power. The samples were amalgamated into a disk applying high pressure (ca.1470.00 MPa) by means of a hydraulic press. The prepared sample was mounted in the cell, which was aligned with the infrared beam of the spectrometer. FTIR spectroscopic measurements were carried out at ambient conditions using a Varian 1000 Scimitar Series spectrophotometer. The FTIR spectra of the polymer samples were recorded using a transparent $\mathrm{KBr}$ pellet for background correction with identical sample holder. The data were processed using the Varian Resolution (version 4.0.5.009) software.

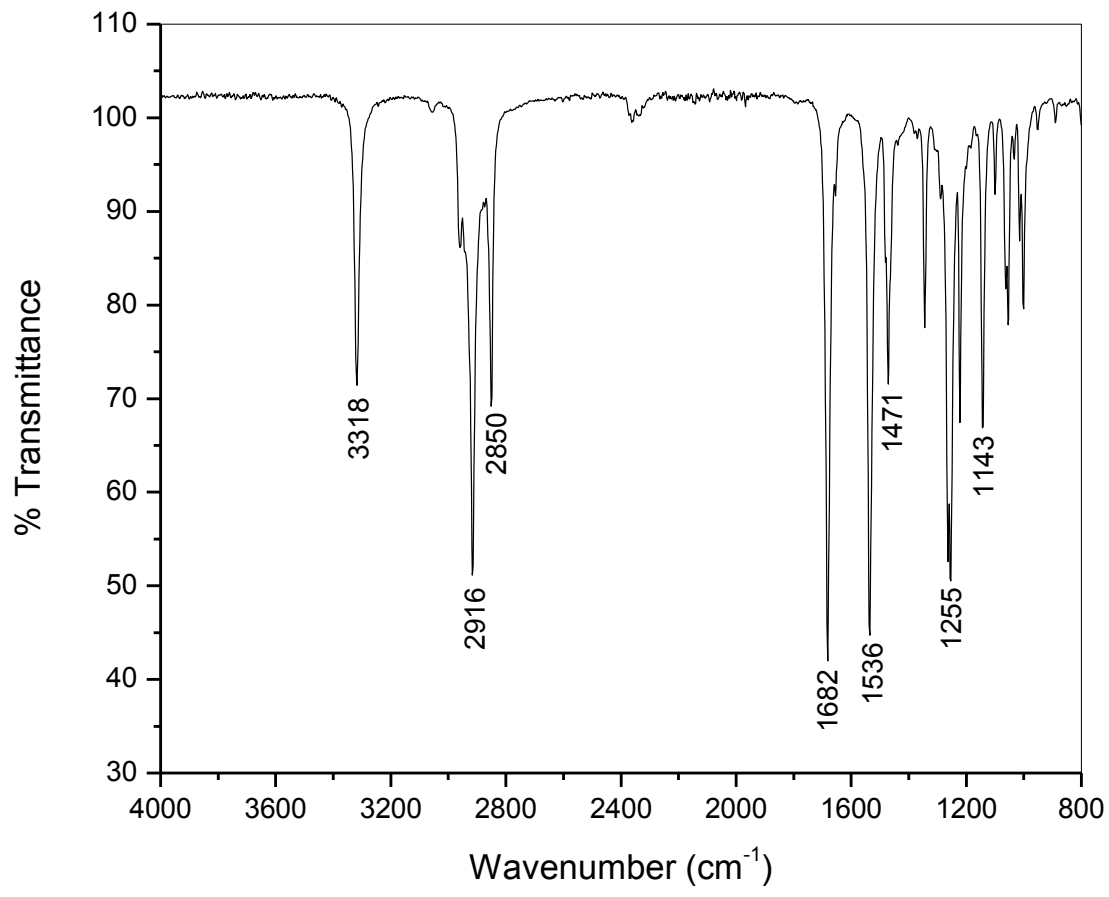

Figure 2.2. FTIR spectra of the $\mathrm{C}_{12}$ biscarbamate. 
The FTIR spectra of all the biscarbamates showed a strong carbonyl stretching vibration (amide I band) at $\sim 1682 \mathrm{~cm}^{-1}$. The band due to $\mathrm{C}=\mathrm{O}$ out-of-plane vibrations was observed at $782 \mathrm{~cm}^{-1}$, which is a characteristic band as it is used as an internal standard in the IR spectra of polyurethanes. ${ }^{16}$ The amide II band, which is due to N-H bending, appears at $1537-1539 \mathrm{~cm}^{-1}$ whereas $\mathrm{N}-\mathrm{H}$ stretching frequencies are recorded at $3317-3336 \mathrm{~cm}^{-}$ ${ }^{1}{ }^{7,8}$ Figure 2.2 represents the FTIR spectra of the $C_{12}$ sample and the stretching frequencies of some of the bands observed in its spectrum are listed in Table 2.2.

Table 2.2. Stretching frequencies of the IR bands of the representative $C_{12}$ biscarbamate.

\begin{tabular}{ll}
\hline Frequency $\left(\mathrm{cm}^{-1}\right)$ & \multicolumn{1}{c}{ Band Assignment } \\
\hline 3318 & $\mathrm{~N}-\mathrm{H}$ stretching \\
2959 & $\mathrm{CH}_{3}$ asymmetrical stretching \\
2916 & $\mathrm{CH}_{2}$ asymmetrical stretching \\
2850 & $\mathrm{CH}_{2}$ symmetrical stretching \\
1682 & $\mathrm{C}=\mathrm{O}$ stretching vibrations \\
1536 & $\mathrm{CHN}$ group vibration \\
1471 & $\mathrm{CH}$ bending \\
1254 & $\mathrm{C}-\mathrm{O}$ stretching \\
1143 & $\mathrm{C}-\mathrm{N}$ stretching \\
781 & $\mathrm{C}=\mathrm{O}$ out-of-plane vibration \\
718 & $(\mathrm{CH})_{n}-$ in - phase rocking \\
\hline
\end{tabular}




\subsubsection{The ${ }^{1} \mathrm{H}$ NMR Spectra of the Biscarbamates}

Nuclear magnetic resonance (NMR) is a spectroscopic technique that detects the energy absorbed by changes in the nuclear spin state (I). Although isotopes can have a variety of values for I (including zero), the most useful for spectroscopy are those nuclei which have $\mathrm{I}=1 / 2$. This includes isotopes ${ }^{1} \mathrm{H}{ }^{13} \mathrm{C},{ }^{15} \mathrm{~N},{ }^{17} \mathrm{O}$, and ${ }^{19} \mathrm{~F}$. When a nucleus with $\mathrm{I}=1 / 2$ is placed in a magnetic field (Figure 2.3), it can either align itself with the field (lower energy) or against it (higher energy). If radio waves are applied, nuclei in the lower energy state can absorb the energy and jump to the higher energy state. We can observe either the absorption of energy, or the subsequent release of energy as the nucleus "relaxes" back to the lower energy state. Traditionally this was done by scanning slowly through a range of radio wave frequencies (this is called continuous wave, CW). However this has largely been replaced by the faster Fourier Transform (FT) method where one big, broad pulse of radio waves is used to excite all nuclei, then the results are analyzed by computer.

The magnetic field strength at the nucleus differs slightly from the applied field, $\mathrm{B}_{\mathrm{o}}$, because of shielded by the electron density surrounding the nucleus. This shielding is due to precession of electrons under the influence of the applied magnetic field, which generates an additional magnetic field that usually opposes the externally applied magnetic field. As the electron density around the nucleus increases, the effective field decreases, leading to lower resonance frequencies. Since the resonance frequency is due to the chemical environment of the nuclear spin, the observed frequency is referred to as a chemical shift. Due to differences in shielding, different spins will experience different values of local magnetic field, giving rise to shifts in their frequencies. Chemical shift 
reference standards, such as tetramethylsilane, $\left(\mathrm{CH}_{3}\right)_{4} \mathrm{Si}$, were chosen because their protons are highly shielded. ${ }^{18}$

The case of the spin- $1 / 2$ nucleus

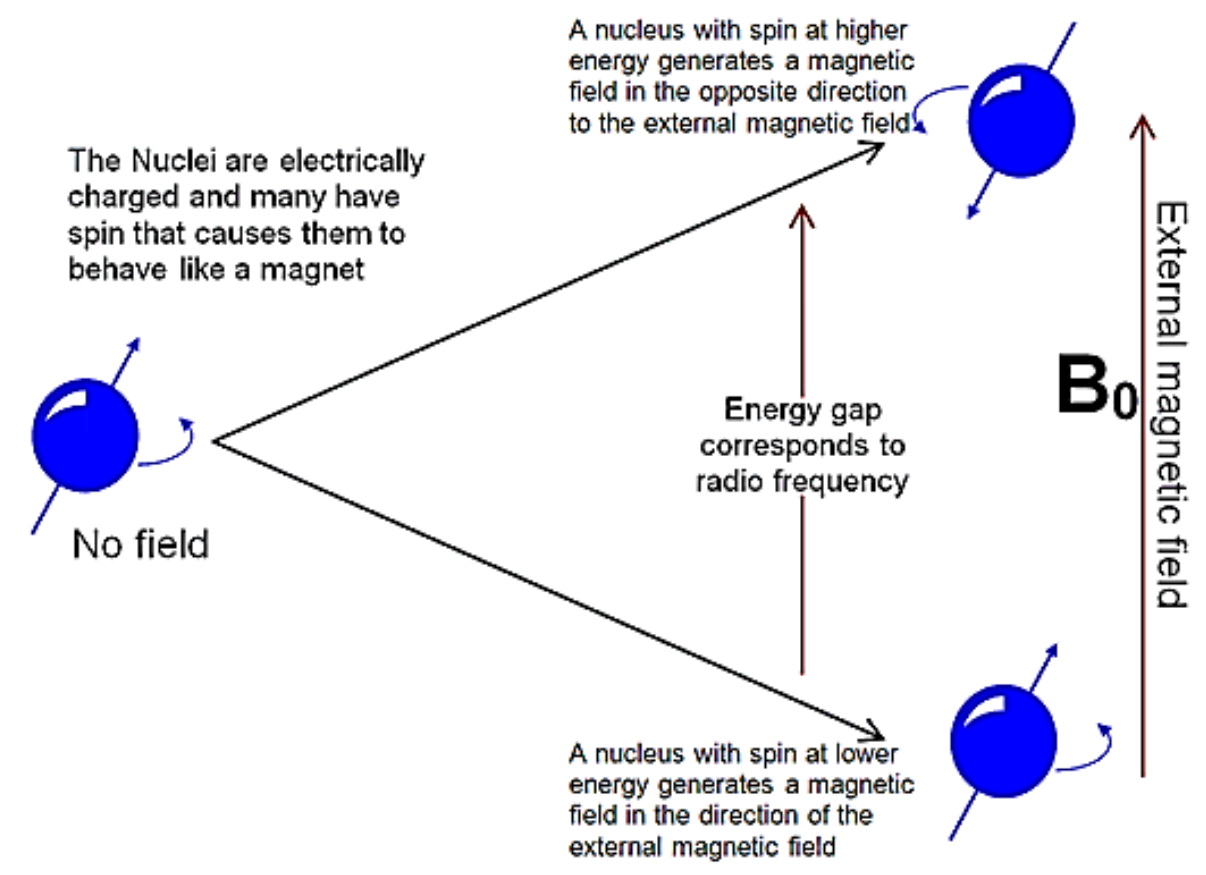

Figure 2.3 The spin- $1 / 2$ nuclei include the most common ${ }^{1} \mathrm{H}$ NMR nucleus, as well as many other nuclei such as ${ }^{13} \mathrm{C},{ }^{15} \mathrm{~N}$ and ${ }^{31} \mathrm{P}$. Taken from ref 17.

The ${ }^{1} \mathrm{H}$ NMR spectra were taken in deuterated chloroform using a $300 \mathrm{MHz}$ spectrometer (Bruker). Tetramethylsilane was used for internal calibration. The ${ }^{1} \mathrm{H}$ NMR spectrum of $\mathrm{C}_{12}$ is shown in Figure 2.4. From the spectrum below, the $\mathrm{CH}_{3}$ protons show a triplet around $\delta 0.90 \mathrm{ppm}$. N-CH 2 absorption at around $\delta 3.18 \mathrm{ppm}$ splits into a quartet by coupling with the $\mathrm{N}-\mathrm{H}$ and neighboring $\mathrm{CH}_{2}$ proton. The $\mathrm{N}-\mathrm{H}$ proton shows a broad signal around $\delta 4.63 \mathrm{ppm}$. The signal is affected by the magnetic and electric properties of ${ }^{14} \mathrm{~N}$ 
nucleus in such way as to make it appear as a broad signal. ${ }^{14} \mathrm{The}-\mathrm{O}-\mathrm{CH}_{2}$ protons show a triplet around $\delta 4.05 \mathrm{ppm}$. The $\mathrm{CH}_{2}-\mathrm{C}-\mathrm{O}$ - protons show a triplet at $\delta 1.6 \mathrm{ppm}$ and the $\mathrm{CH}_{2}$ $\mathrm{C}-\mathrm{N}$ - protons show another triplet at $\delta 1.5 \mathrm{ppm}$. The remaining $\mathrm{CH}_{2}$ protons show a multiplet around $\delta 1.27 \mathrm{ppm}$.

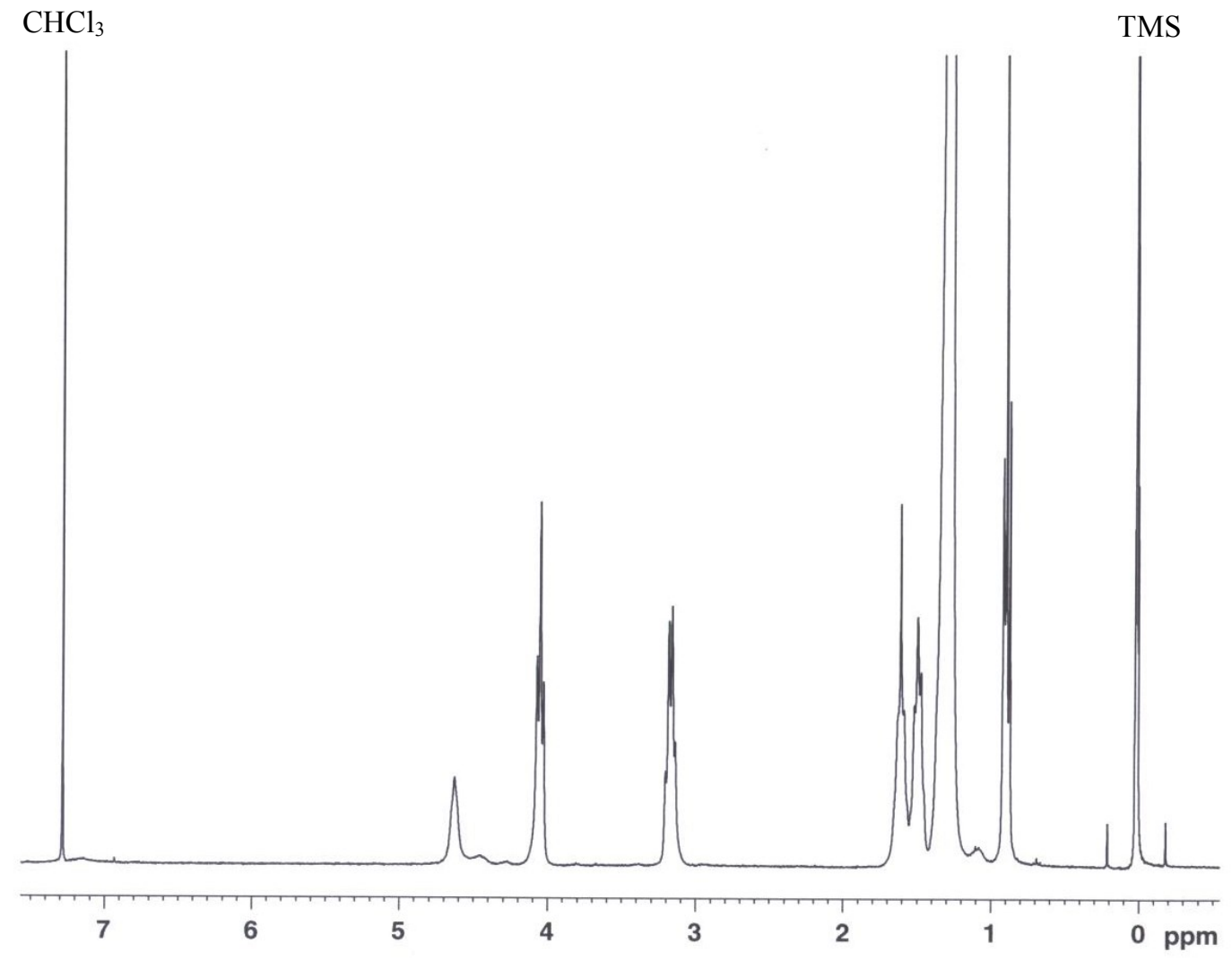

Figure 2.4. ${ }^{1} \mathrm{H}$ NMR spectra of $\mathrm{C}_{12}$ biscarbamate.

\subsection{Experimental methods}

Thermal behaviour of the biscarbamates, their blends and organogels was investigated by differential scanning calorimetry (DSC). Optical microscopy (OM) and 
scanning electron microscopy (SEM) were used to investigate the morphology. Powder Xray diffraction (XRD) and Fourier transform infrared spectroscopy (FTIR) were used to inspect for any degradations in the samples. The following subsections briefly describe the basic principles of the methods and the instruments used for this study.

\subsubsection{Differential Scanning Calorimetry}

Differential scanning calorimetry (DSC) is a technique that is used very often for quantitative studies of thermal transitions in polymers, metals, organic materials, ceramics, and composites. The sample and an inert reference are heated, usually in a nitrogen atmosphere, and thermal transitions in the sample are detected and measured. A schematic representation of the mechanism of a DSC cell is shown in Figure 2.5. The sample and the reference are provided with individual heaters. Energy is supplied to keep the sample and reference temperatures constant. The electrical power difference between sample and reference $(\mathrm{d} \Delta \mathrm{Q} / \mathrm{dt})$ is recorded as a function of temperature. When the sample undergoes a thermal transition, the power to the two heaters is adjusted to maintain their temperatures. The temperature is plotted along one axis of an $\mathrm{x}-\mathrm{y}$ recorder and a signal proportional to the power difference is plotted along the second axis of the recorder. This heat flow/temperature data provides valuable information of physical/chemical properties of the materials such as: the crystalline melting temperature $\left(\mathrm{T}_{\mathrm{m}}\right)$, the crystallization temperature $\left(\mathrm{T}_{\mathrm{c}}\right)$, the heat of fusion $\left(\mathrm{H}_{\mathrm{f}}\right)$, the glass transition temperature $\left(\mathrm{T}_{\mathrm{g}}\right)$, the specific heat capacity $\left(\mathrm{C}_{\mathrm{p}}\right)$, the gel-to-solution temperature $\left(\mathrm{T}_{\text {gel-sol }}\right)$, and the gelation temperature $\left(\mathrm{T}_{\text {gel }}\right)$. 


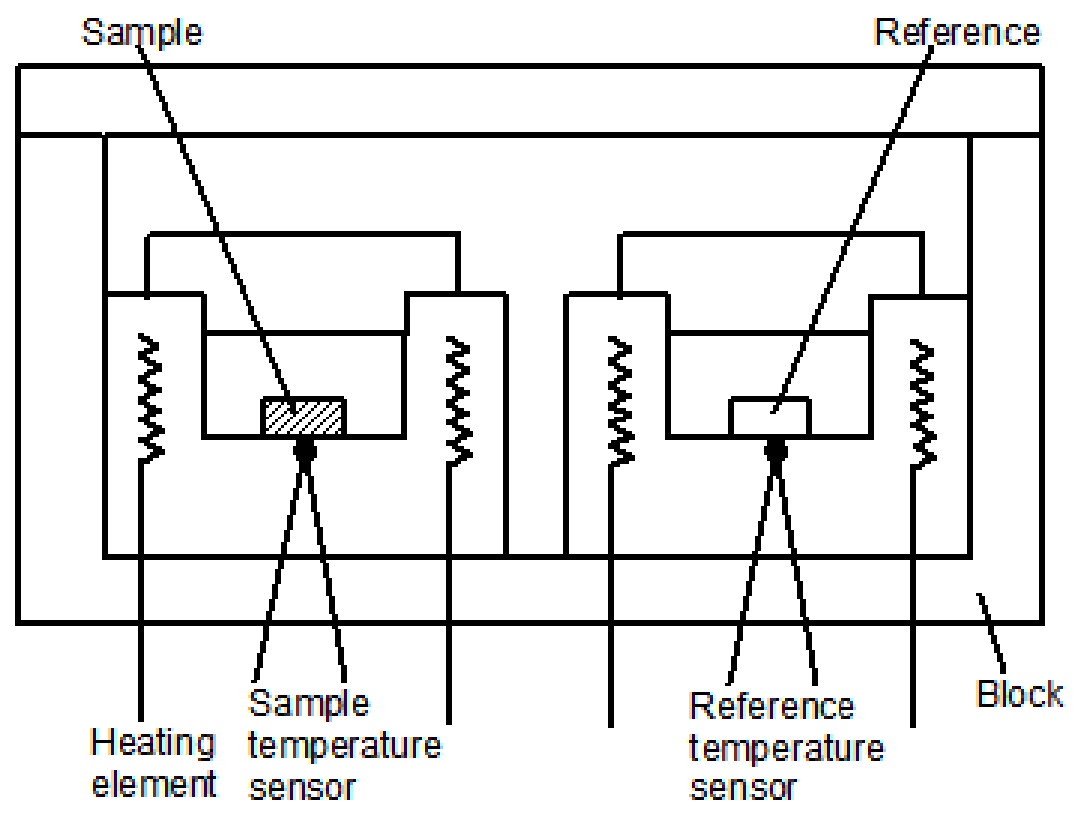

Figure 2.5. Schematic representation of a DSC cell.

In this study, thermal analysis was performed using a TA Instruments 2010 differential scanning calorimeter at a heating rate of $5{ }^{\circ} \mathrm{C} / \mathrm{min}$. A few select samples were heated at a heating rate of $1{ }^{\circ} \mathrm{C} / \mathrm{min}$. The instrument was calibrated for temperature and energy with indium as certified reference materials. DSC traces were recorded with about 7-10 $\mathrm{mg}$ of the biscarbamate and their blend samples, in a crimped aluminum pan $(20 \mu \mathrm{L})$ under a nitrogen atmosphere. Traces for wet organogel samples were recorded once again under the flow of nitrogen with about $30-45 \mathrm{mg}$ of the samples in a crimped pan $(50 \mu \mathrm{L})$ while making sure no solvent or condensation would escape. The uncertainty in the measurements was $\pm 1{ }^{\circ} \mathrm{C}$ for the melting and crystallization points and $\pm 2 \mathrm{~J} / \mathrm{g}$ for the heat of fusion and crystallization. The software used to analyze the thermographs is TA Instruments Universal Analysis 2000 (version 3.9A). 


\subsubsection{Optical Microscopy}

Optical microscopy is a useful and relatively inexpensive technique to produce magnified visual or photographic images of small objects. It can also be used to observe the behaviour or other changes of a small system in a variety of applied conditions and circumstances. Modern microscopes can resolve surface characteristics of the order of $2000 \AA$ and can be equipped with a range of accessories and features such as for various systems of illumination or technique including heating-stage, phase-contrast, interferometry, photomicrography, fluorescence, ultra-violet light, and polarizations. ${ }^{19}$

For our study, the spherulitic structure of materials is not visible to the naked eye, so the use of an optical microscope is necessary. Polarized light was used to examine the optical characteristics of the biscarbamates samples and, more specifically, signs of birefringence of the spherulites (i.e., the structures that have two different refractive indices at right angles to one another) were examined. The sign of the spherulite birefringence depends on the orientation of the optic axes of the crystalline domain with respect to the polarization direction of the incident light. The sign of the spherulite can be determined by using a sensitive tint plate ( $\lambda$ plate) that can add to the path difference of the spherulite or subtract from it, depending on whether the birefringent units in the spherulite are oriented parallel to the plate or perpendicular to it. This means that one pair of quadrants of a spherulite will be raised up the scale of colors and the other will be lowered. Therefore, with spherulites showing only first order white, which is normal in a thin section, one pair will be raised to blue and the other lowered to orange. ${ }^{20}$ The housing of the $\lambda$ plate has an arrow showing the polarization direction. If the quadrants of the spherulites parallel to the arrow appears orange and the other quadrants appear blue, the spherulites are called 
negative spherulites and vice versa are called positive spherulites. Depending on the thickness of the sample, the colors may vary. Figure 2.6 shows the appearance of the positive and negative spherulite under optical microscope.
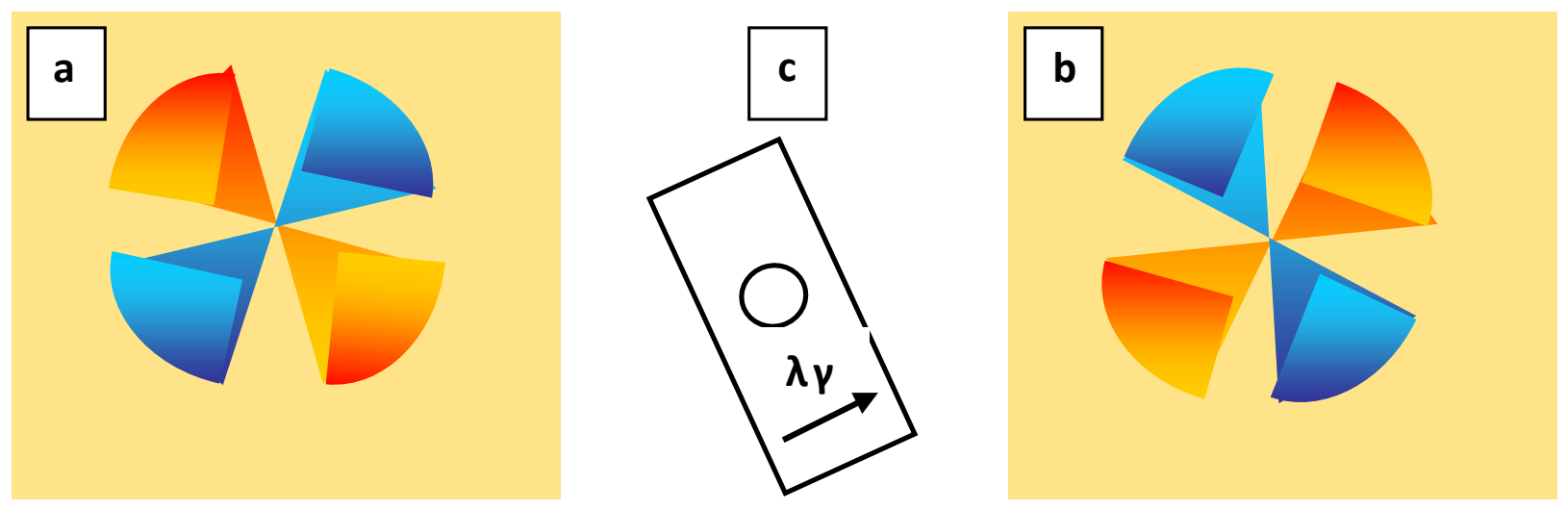

Figure 2.6. Schematic presentation of a (a) positive spherulite, (b) negative spherulite, and (c) tint $\lambda$ plate.

In our studies, optical microscopy was used to investigate the morphology of the neat biscarbamate, the blends and their precipitation from solution, the prepared gels and xerogels. The optical micrographs were recorded using a Zeiss Axioplan polarized optical microscope, equipped with a Linkam hot stage for variable temperature optical microscopy. All the optical micrographs were taken in transmission mode. Northern Eclipse (version 6.0 and 8.0) image processing software was used to record the images as well as to calculate (where it was possible) the size of the features, e.g., fibres, sheets, spherulites, etc. 
The samples for the optical microscopy of the neat biscarbamates and their melt blends were prepared by melting a small amount of the material on the microscope slide at a temperature $20^{\circ} \mathrm{C}$ higher than its melting point (in case of blend, $20^{\circ} \mathrm{C}$ higher than the melting temperature of the higher melting component), holding it isothermally for $10 \mathrm{~min}$ to remove any morphological history and then cooling it down slowly to room temperature at the rate of $5{ }^{\circ} \mathrm{C} / \mathrm{min}$. Another set of samples was prepared following the same procedure, but this time the samples were quenched from the melt, instead of slow cooling. The samples for the solution blends were prepared by placing a drop of the chloroform solution sample at $60^{\circ} \mathrm{C}$ on a concave microscope slide, sealing the area around the solvent and placing a safety cover over it. The images were taken at various temperatures controlled by a hot stage and an air cooling system.

\subsubsection{Scanning Electron Microscopy}

A scanning electron microscope (SEM) scans a focused electron beam over a surface to create an image. The electrons in the beam interact with the sample, producing various signals that can be used to obtain information about the surface topography and composition. A modern light microscope has a maximum magnification of about 1000x. The resolving power of the microscope was not only limited by the number and quality of the lenses but also by the wavelength of the light used for illumination. ${ }^{21}$ White light has wavelengths from 400 to 700 nanometers $(\mathrm{nm})$. The average wavelength is $550 \mathrm{~nm}$ which results in a theoretical limit of resolution (not visibility) of the light microscope in white light of about $200-250 \mathrm{~nm}$. The electron microscope was developed when the wavelength became the limiting factor in light microscopes. Electrons have much shorter wavelengths, enabling better resolution. 
The main SEM components include: a source of electrons, a column down which electrons travel with electromagnetic lenses, an electron detector, and a sample chamber (Figure 2.7). ${ }^{21}$ Electrons are produced at the top of the column, accelerated down and passed through a combination of lenses and apertures to produce a focused beam of electrons which hits the surface of the sample. The sample is mounted on a stage in the chamber area and, unless the microscope is designed to operate at low vacuums, both the column and the chamber are evacuated by a combination of pumps. The level of the vacuum will depend on the design of the microscope. The position of the electron beam on the sample is controlled by scan coils situated above the objective lens. These coils allow the beam to be scanned over the surface of the sample. This scanning beam enables information about a defined area on the sample to be collected. As a result of the electronsample interaction, a number of signals are produced. These signals are then detected by appropriate detectors.

The SEM produces images by scanning the sample with a high-energy beam of electrons. As the electrons interact with the sample, they produce secondary electrons, backscattered electrons, Auger electrons, characteristic X-rays and photons of various energies. These signals are collected by one or more detectors to form images which are then displayed on the computer screen. The resulting images have great depth of fields and a remarkable three-dimensional appearance due to the confinement of the secondary electrons to a volume near the beam impact area of the sample and the shadow relief effect of the secondary electron contrast. The maximum resolution obtained in an SEM depends on multiple factors, like the electron spot size and interaction volume of the electron beam with the sample. While it cannot provide atomic resolution, some SEMs can achieve 
resolution below $1 \mathrm{~nm} \cdot{ }^{21,22}$ Typically, modern full-sized SEMs provide resolution between 1-20 nm whereas desktop systems can provide a resolution of $20 \mathrm{~nm}$ or more. For gel fibres where the average length can reach to the millimeter range and the average width is around a few micrometers. Although it would be interesting to look at the structure details in these ranges, high resolution images were not necessary to for the purposes of this thesis.

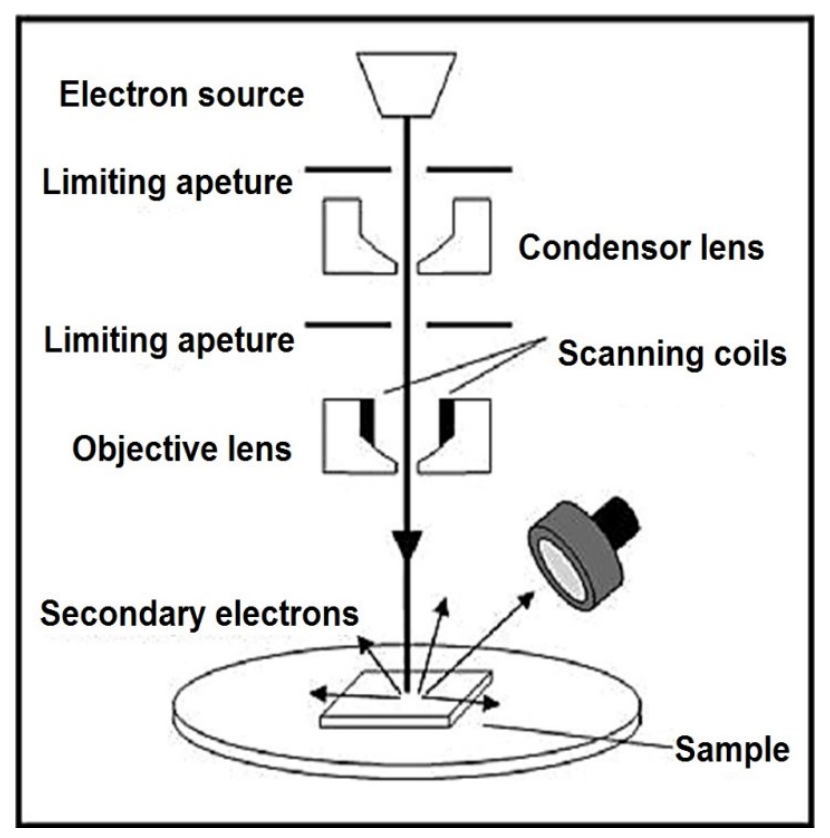

Figure 2.7. Schematic diagram of a scanning electron microscope. Taken from ref 22.

SEM images of the xerogels were obtained using a JEOL JSM-6400 scanning electron microscope. They are then mounted on carbon tape and sputter coated with 80:20 $\mathrm{Au} / \mathrm{Pd}$ alloy. SEM images were captured at an accelerating voltage ranging from 5 to 20 $\mathrm{kV}$. Vega TC software were used for the microscope manipulation and capturing the images. 


\section{$\underline{\text { 2.4.4 X-ray Diffraction }}$}

The X-ray diffraction (XRD) is a powerful, non-destructive method for investigating orderly arrangement of atoms or molecules through the interaction of electromagnetic radiation to give interference effects with structures comparable in size to the wavelength of radiation. ${ }^{23}$ Crystalline substances are composed of parallel planes of atoms separated by a distance known as d-spacing. Diffraction occurs when electromagnetic radiation enters such a crystalline substance and is scattered from the different planes of that substance. The wavelengths of X-rays are comparable to interatomic distances in crystals. ${ }^{24}$ For complete information on the molecular ordering in a crystalline or semi-crystalline material, it is necessary to obtain a single crystal of the compound. ${ }^{25}$ However, when a single crystal is not available, powder X-ray diffraction can provide valuable information. Valuable information can be extracted by using this instrument, such as the d-spacing, the crystallite size and the percent crystallinity. In powder X-ray diffraction, the reflections are obtained from several microcrystals with random orientation.

The basic equation of X-ray diffraction is the Bragg formula:

$$
2 d \sin \theta=n \lambda
$$

where $\lambda$ is the wavelength of $\mathrm{X}$-ray radiation, $\mathrm{d}$ is the distance between atomic planes, $\theta$ the angle of incidence of the X-ray beam on the atomic planes and $n(1,2,3 \ldots, n)$ is the order of the reflection. In real cases, higher order reflection corresponding to $n>1$ are extremely weak in intensity. Hence only those corresponding to $n=1$ are observed. Constructive interference only occurs for certain $\theta$ values correlating to a (hkl) plane, 
specifically when the path difference is equal to $\mathrm{n}$ wavelengths (Figure 2.8).

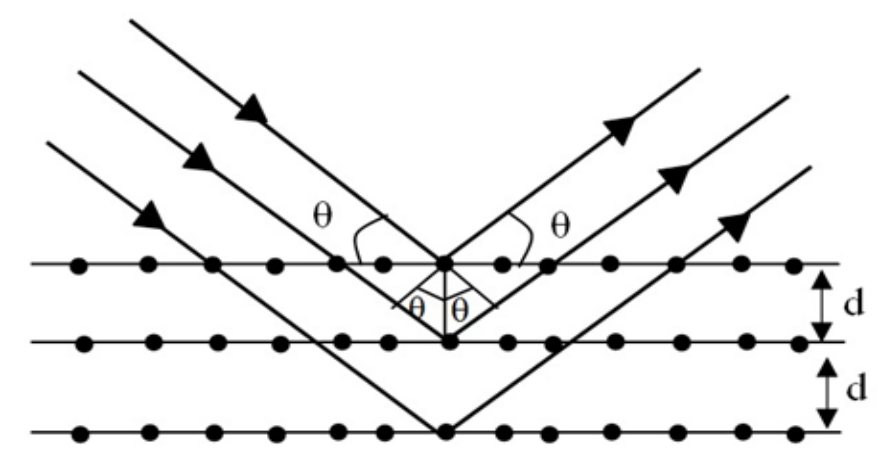

Figure 2.8. Bragg construction illustrating the principle of diffraction where $d$ is the spacing between two atomic planes.

Rearrangement of Bragg's equation gives the $d$-spacing as

$$
d=\frac{\lambda}{2 \sin \theta}
$$

Equation 2.2 shows that when the distance between the two planes in the lattice is large, the scattering angle (29) has to be small to produce a diffraction pattern. The diffraction obtained at angles smaller than $2^{\circ}(\mathrm{d}>\sim 50 \AA)$ is called Small Angle X-ray Scattering (SAXS). The diffraction obtained at all larger angles, theoretically extending up to $180^{\circ}$ is called Wide Angle X-ray Diffraction (WAXD). WAXD provides information about the spatial arrangement of the atoms, whereas, SAXS provides the information about domain arrangement (e.g. long spacing).

In our studies, XRD data were collected within the range of $2^{\circ} \leq 2 \theta \leq 45^{\circ}$ for all of 
the samples using a Philips automated powder diffractometer, Model PW 1710. Nickelfiltered $\mathrm{Cu} \mathrm{K} \alpha$ radiation $(\lambda=1.542 \AA)$. The possible presence of texture was checked by taking additional diffractograms with the sample turned in the plane of measurement by $90^{\circ}$. Figure 2.9 shows a schematic of the Bragg diffraction with the diffractometer. ${ }^{26} \mathrm{MDI}$ Datascan 3.2 software (Materials Data Inc., Livermore, CA) was used for data collection. The result analysis was performed using MDI Jade 5.0 XRD Pattern Processing software.

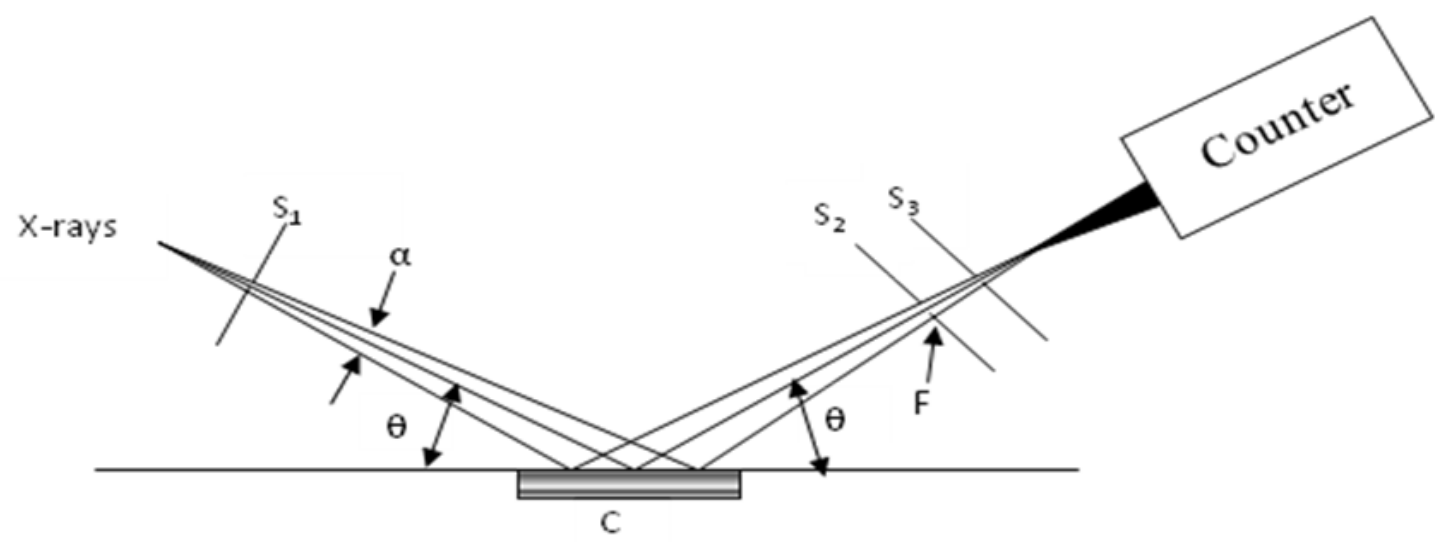

Figure 2.9. Schematic of the Bragg diffraction of the powder X-ray diffractometer used for the present study. Here, $S_{1}, S_{2}$ and $S_{3}$ are the divergence slit, receiving slit and scatter slit respectively; $\alpha=1-4^{\circ}$.

Percent crystallinity $\left(\mathrm{X}_{\mathrm{c}}\right)$ can be measured by using powder XRD. It is the ratio of crystalline peaks to the sum of the crystalline and amorphous intensities and can be calculated using the formula:

$$
X_{C}=\frac{I_{C}}{I_{C}+I_{A}} \times 100
$$


where $I_{\mathrm{C}}$ is the crystalline area under the peak and $I_{\mathrm{A}}$ is the amorphous area as shown in Figure 2.10.

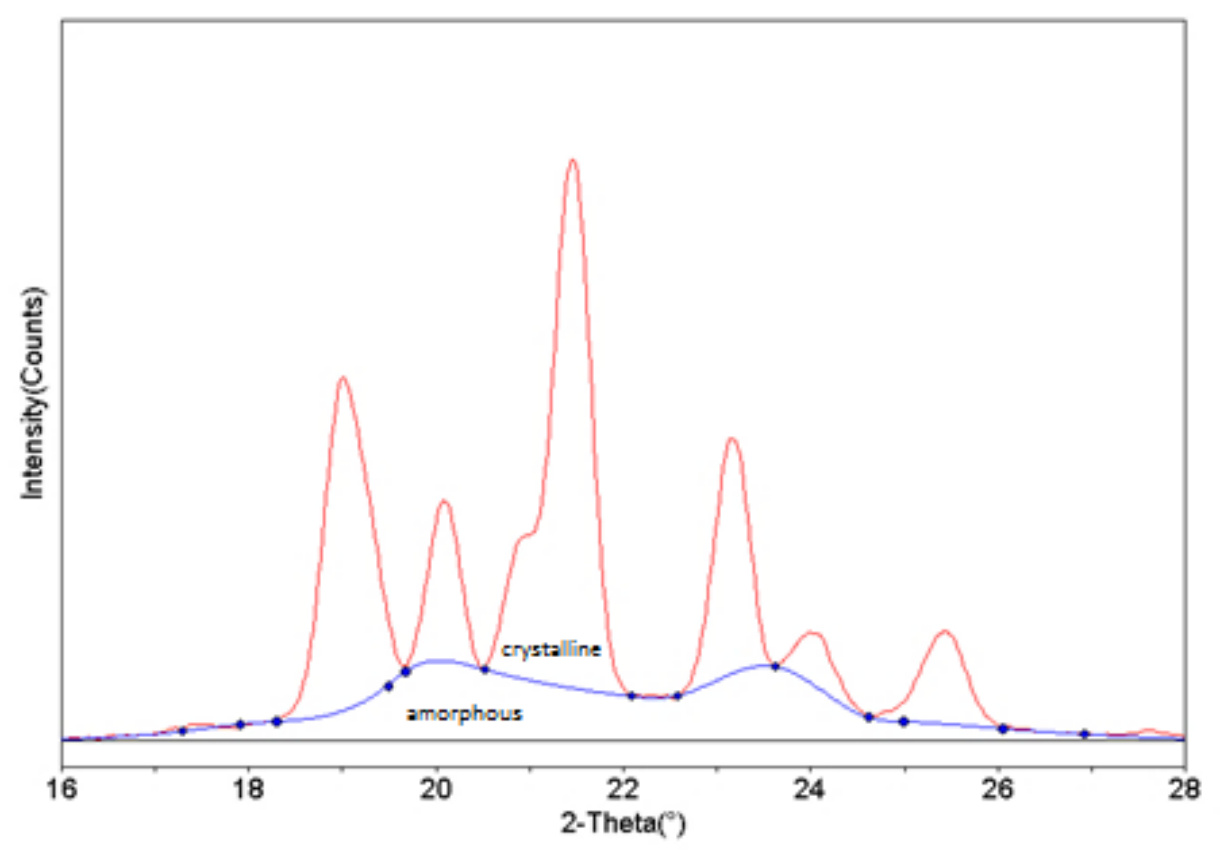

Figure 2.10. Schematic showing the crystalline and amorphous peak area used for calculating percent crystallinity.

\subsubsection{Computational Chemistry}

Computational chemistry is the application of mathematical models and simulations to solve chemical and biological problems. Computational technologies have a broad range of applications, from molecular modeling to the simulation and control of chemical processes. ${ }^{27}$ The aspects of computational technology that are most critical to the 
chemical industry include computational molecular science, ${ }^{28}$ process modeling and simulation, ${ }^{29}$ optimization of operations and process control, ${ }^{30}$ and computational fluid dynamics. ${ }^{31}$ The field of computational chemistry, broadly defined, includes computational molecular science, empirical correlations such as linear free energy relationships and Quantitative Structure Property Relationships (QSPR), and aspects of process modeling and simulation. ${ }^{32}$

In the previous studies of our group, molecular modelling was used as a tool to predict the conformation of biscarbamate molecules and the intermolecular forces responsible for the association process. ${ }^{33}$ In this work, chemical properties of biscarbamates were derived from simulations. Simple molecular Modeling was performed using HyperChem ${ }^{34}$ (from Hypercube Inc.) (version 7.5) and Spartan ES software. ${ }^{35}$ Geometry optimization of the molecular structures was done using the MM+ force field. 


\subsection{References}

(1) Goodbrand, B.; Boils, D. C.; Sundararajan, P. R.; Wong, R. W. US Patent $6,414,051,2002$.

(2) Khan, M. K.; Sundararajan, P. R. Molecular Selectivity and Immiscibility during the Crystallization of Mixtures of a Set of Homologous Self-Assembling Molecules. J. Phys. Chem. B 2008, 112 (14), 4223-4232.

(3) Moniruzzaman, M.; Sundararajan, P. R. Low Molecular Weight Organogels Based on Long-Chain Carbamates. Langmuir 2005, 21 (9), 3802-3807.

(4) Khanna, S.; Khan, M. K.; Sundararajan, P. Influence of Double Hydrogen Bonds and Alkyl Chains on the Gelation of Nonchiral Polyurethane Model Compounds: Sheets, Eaves Trough, Tubes and Oriented Fibers. Langmuir 2009, 25 (22), 1318313193.

(5) Khan, M. K.; Sundararajan, P. Encapsulation of Dye Molecules and Nanoparticles in Hollow Organogel Fibers of a Nonchiral Polyurethane Model Compound. Chem. Eur. J. 2011, 17 (4), 1184-1192.

(6) Erickson, B. Product Review: Standardizing the World with Microwaves. Anal. Chem. 1998, 70 (13), 467A - 471A.

(7) Dyer, J. R. Applications of Absorption Spectroscopy of Organic Compounds. 1965.

(8) Socrates, G. Infrared and Raman Characteristic Group Frequencies: Tables and Charts; John Wiley \& Sons, 2004. 
(9) Finlayson-Pitts, B. J.; Pitts Jr, J. N. Chemistry of the Upper and Lower Atmosphere: Theory, Experiments, and Applications; Academic press, 1999.

(10) Hinsmann, P.; Frank, J.; Svasek, P.; Harasek, M.; Lendl, B. Design, Simulation and Application of a New Micromixing Device for Time Resolved Infrared Spectroscopy of Chemical Reactions in Solution. Lab Chip 2001, 1 (1), 16-21.

(11) Ogawa, T.; Carlson, G. A.; Pimentel, G. C. Reaction Rate of Trifluoromethyl Radicals by Rapid Scan Infrared Spectroscopy. J. Phys. Chem. 1970, 74 (10), 2090-2095.

(12) Osland, R. C. J. Principles and Practices of Infrared Spectroscopy; Pye Unicam, 1985.

(13) Pavia, D.; Lampman, G.; Kriz, G.; Vyvyan, J. Introduction to Spectroscopy; Cengage Learning, 2008.

(14) Kalsi, P. S. Spectroscopy of Organic Compounds; New Age International, 2007.

(15) Skoog, D. A.; Leary, J. J. Principles of Instrumental Analysis. Clin. Chem. Ed. 1994, $40(8), 1612$.

(16) Furer, V. L. The IR Spectra and Hydrogen Bonding of Toluene-2, 6-Bis (methyl) and 4, 4'-Diphenylmethane-Bis (methyl) Carbamates. J. Mol. Struct. 2000, 520 (1), $117-123$.

(17) Bushong, S. C.; Clarke, G. Magnetic Resonance Imaging: Physical and Biological Principles; Elsevier Health Sciences, 2013. 
(18) Mohrig, J. R.; Hammond, C. N.; Schatz, P. F. Techniques in Organic Chemistry; Macmillan, 2010.

(19) Robinson, P. C.; Bradbury, S. Qualitative Polarized-Light Microscopy; Bios Scientific Pub Limited, 1992; Vol. 9.

(20) Hammond, C.; Hammond, C. The Basics of Crystallography and Diffraction; Oxford University Press Oxford, 2009.

(21) Egerton, R. Physical Principles of Electron Microscopy: An Introduction to TEM, SEM, and AEM; Springer Science \& Business Media, 2006.

(22) Kalantar-zadeh, K.; Fry, B. Nanotechnology-Enabled Sensors; Springer Science \& Business Media, 2007.

(23) Goldstein, J.; Newbury, D. E.; Echlin, P.; Joy, D. C.; Romig Jr, A. D.; Lyman, C. E.; Fiori, C.; Lifshin, E. Scanning Electron Microscopy and X-Ray Microanalysis: A Text for Biologists, Materials Scientists, and Geologists; Springer Science \& Business Media, 2012.

(24) Klug, H. P.; Alexander, L. E. X-Ray Diffraction Procedures. 1954.

(25) Kim, S. H.; Lee, C. M.; Kafle, K. Characterization of Crystalline Cellulose in Biomass: Basic Principles, Applications, and Limitations of XRD, NMR, IR, Raman, and SFG. Korean J. Chem. Eng. 2013, 30 (12), 2127-2141.

(26) Alexander, L. E. X-Ray Diffraction Methods in Polymer Science. 1969.

(27) Hay, S.; Scrutton, N. S. Examining the Importance of Dynamics, Barrier 
Compression and Hydrogen Tunnelling in Enzyme Catalysed Reactions. Procedia Chem. 2011, 3 (1), 306-315.

(28) Kam, K. M.; Tade, M. O.; Rangaiah, G. P.; Tian, Y. C. Strategies for Enhancing Geometric Nonlinear Control of an Industrial Evaporator System. Ind. Eng. Chem. Res. 2001, 40 (2), 656-667.

(29) Prat-Resina, X.; Caldwell, B. JCE Concept Connections: Computational Molecular Modeling. J. Chem. Educ. 2009, 86 (8), 958.

(30) Ramadesigan, V.; Northrop, P. W. C.; De, S.; Santhanagopalan, S.; Braatz, R. D.; Subramanian, V. R. Modeling and Simulation of Lithium-Ion Batteries from a Systems Engineering Perspective. J. Electrochem. Soc. 2012, 159 (3), R31-R45.

(31) Tu, J.; Yeoh, G. H.; Liu, C. Computational Fluid Dynamics: A Practical Approach; Butterworth-Heinemann, 2012.

(32) Ramachandran, K. I.; Deepa, G.; Namboori, K. Computational Chemistry and Molecular Modeling: Principles and Applications; Springer Science \& Business Media, 2008.

(33) Khanna, S.; Moniruzzaman, M.; Sundararajan, P. R. Influence of Single versus Double Hydrogen-Bonding Motif on the Crystallization and Morphology of SelfAssembling Carbamates with Alkyl Side Chains: Model System for Polyurethanes. J. Phys. Chem. B 2006, 110 (31), 15251-15260.

(34) Release, H. 7.5 for Windows, Molecular Modeling System, Hypercube. Inc. http//www. hyper. com 2002. 
(35) Spartan, E. S. 02; Wavefunction Inc. Irvine, $C A$. 


\section{CHAPTER 3}

Inhibiting the Self-Sorting Behaviour in the Blends of a Homologous Set of Biscarbamates 


\section{CHAPTER III: INHIBITING THE SELF-SORTING BEHAVIOUR IN THE BLENDS OF A HOMOLOGOUS SET OF BISCARBAMATES}

\subsection{Introduction}

In a previous study, ${ }^{1}$ it was shown that melt-mixing two biscarbamates with different size side chains, both with the same distance between carboxylic groups, does not lead to miscible blends. Although the central part of the molecules is the same, the difference in the side chain length causes a disruption in the packing of the molecules and changes in the melting temperature and crystallinity. In addition to a reduction in the spherulite size, the rate of growth of the spherulites is also affected. This behaviour is more pronounced when the difference between the lengths of the side chains is large. These molecules show an affinity for itself giving rise to narcissistic self-sorting despite the close similarities between the different biscarbamates. This proves to be interesting as these molecules are an example of the first non-chiral self-sorting systems reported as of yet.

Self-sorting phenomenon is a scientifically interesting and important phenomenon that has been studied extensively. This behaviour could be used effectively for applications such as chiral separations. However, it could also be disadvantageous, when miscible blends are required for certain applications for tuning material properties. In this chapter, we examine various approaches to supress the self-sorting of the biscarbamates and promote their miscibility. There are many factors that control the miscibility of a blend: the size, the melting and crystallization temperature, and the rate of crystallization. ${ }^{2-5}$ It was hypothesized that modifying the rate of crystallization can easily be used to control the miscibility for a melting blend of two samples of similar nature. In an attempt to 
produce a miscible blend of two biscarbamates, we attempted to crystallize the sample from a solvent in order to negate the large melting temperature difference. Only the dissolution and precipitation temperatures of the samples (Table 3.1) will affect the sample preparation. In doing so, the crystals precipitate together from the solvent to limit the selfsorting effect. This will be referred to as solvent mixing.

Table 3.1. Temperatures of dissolution and precipitation of various biscarbamates from chloroform. All the solutions were prepared using the same amount of substrate and solvent.

\begin{tabular}{lll}
\cline { 2 - 3 } & $\mathrm{T}_{\text {dissolution }}\left({ }^{\circ} \mathrm{C}\right)$ & $\mathrm{T}_{\text {precipitation }}\left({ }^{\circ} \mathrm{C}\right)$ \\
\hline $\mathrm{C}_{18}$ & 55.5 & 49.2 \\
$\mathrm{C}_{16}$ & 50.8 & 39.0 \\
$\mathrm{C}_{12}$ & 42.3 & 17.0 \\
$\mathrm{C}_{8}$ & 23.8 & 8.0 \\
$\mathrm{C}_{4}$ & 17.5 & -8.5 \\
\hline
\end{tabular}

In this chapter, we will discuss the effects of the presence of solvent on the biscarbamate blends. We will examine quantitatively the blends using a differential scanning calorimeter (DSC) and compare the miscibility of these blends with the blends prepared from melt mixing. We will also discuss other methods used to improve the miscibility of the blends in order to produce a fully miscible blend. The assembly of 
materials into ordered structures to render them functional and operational is a crucial aspect of material science. ${ }^{6-9}$ Hence, controlling the morphology is greatly important.

\subsection{Experimental}

\subsubsection{Preparation of Blends}

The synthesis of biscarbamates used in this study has been described before. ${ }^{10-13}$ Briefly, this involves reacting 1,6-diisocyanatohexane $(0.1 \mathrm{~mol})$ with various alcohols $(0.2$ mol) ranging from 1-butanol to 1-octadecanol in toluene, in the presence of 1,4diazabicyclo $[2,2,2]$ octane $(\mathrm{DABCO})$ as the catalyst. Blends of biscarbamates were prepared by melt mixing and solution mixing with $25 / 75,50 / 50$ and $75 / 25$ (wt \%) compositions. Three types of binary blends were prepared: biscarbamates with side chain lengths that are very different $\left(\mathrm{C}_{4} / \mathrm{C}_{18}\right)$, somewhat similar $\left(\mathrm{C}_{8} / \mathrm{C}_{12}\right)$ and very close to each other $\left(\mathrm{C}_{16} / \mathrm{C}_{18}\right){ }^{1}$

Melt mixing was performed by heating pre-weighed mixtures of biscarbamates in a $5 \mathrm{~mL}$ vial at a temperature of $20^{\circ} \mathrm{C}$ above that of the higher melting component with continuous stirring. The low melt viscosity of these samples enabled uniform mixing of the components, with stirring. After 30 minutes, the melt was slowly cooled to room temperature by simply turning off the hotplate. Our previous study ${ }^{1}$ has shown that subsequent melting temperatures were the same whether the sample was slowly cooled or quenched from the melt. The slow-cooling method was used in this work.

Solution mixing was achieved by dissolving $0.1 \mathrm{~g}$ of the biscarbamate mixtures in 
$3 \mathrm{~mL}$ of chloroform in a sealed Pyrex vial at $70-80^{\circ} \mathrm{C}$ with continuous stirring. The blends were precipitated out by cooling the sample at a rate of $5{ }^{\circ} \mathrm{C}$ per minute. Table 1 lists the temperatures at which the biscarbamates dissolve and precipitate. When the precipitation occurred below room temperature, the solution was placed in a refrigerator to cool the solution. The solvent was evaporated out while the precipitated solution remained cold. The blends were then left to dry at room temperature for a day, and then kept under vacuum for another day to form the dry solvent-based blends.

\subsubsection{Characterization Methods}

Thermal analysis was performed using a TA Instruments 2010 differential scanning calorimeter at a heating rate of $5^{\circ} \mathrm{C}$ per minute. The calorimeter was calibrated for temperature and energy with an indium sample as a standard reference material. DSC traces were recorded with about 7-8 $\mathrm{mg}$ of the sample in nitrogen atmosphere. The error in $\mathrm{T}_{\mathrm{m}}$ was less than $1^{\circ} \mathrm{C}$. The software TA Instruments Universal Analysis 2000 (version 3.9A) was used to analyze the data.

The optical microscope images were recorded on a Zeiss Axioplan polarized optical microscope (OM). Northern Eclipse (version 6.0) image processing software was used to capture the image. The samples were prepared by placing a drop of chloroform solution at $60{ }^{\circ} \mathrm{C}$ on a concave microscope slide, sealing the area around the solvent and placing a safety cover over it. The images were taken at various temperatures controlled by a Linkam hot stage and an air cooling system.

X-ray diffraction data were collected in the range of $3^{\circ} \leq 2 \theta \leq 30^{\circ}$ using a Philips automated powder diffractometer, Model PW 1710 with nickel-filtered $\mathrm{CuK}_{\alpha}$ radiation $(\lambda$ 
$=1.542 \AA$ ). MDI Datascan 3.2 software (Materials Data Inc., Livermore, CA) was used for data collection. The analysis was performed using MDI Jade 5.0 XRD Pattern Processing software.

\subsection{Blends by Melt-Mixing}

In this chapter, in the AB-type binary blends, A refers to the biscarbamate with shorter alkyl side chain. The melting temperatures of the blends prepared by solution and melt mixing are listed in Table 3.2. If a blend of two components are miscible (i.e., do not self-sort), the DSC analysis would show a single endotherm, with the melting temperature $\left(T_{m}\right)$ that is intermediate between those of the individual species. ${ }^{14,15}$ The $T_{m}$ would then follow the dotted lines in Figure 3.1, which would indicate miscibility. As a consequence, co-crystallization can be expected.

With the melt blends, it is seen that $\mathrm{C}_{4} / \mathrm{C}_{18}$ blends exhibit two melting temperatures for all compositions, while the $\mathrm{C}_{8} / \mathrm{C}_{12}$ blends show two $T_{\mathrm{m}}$ for the $75 / 25$ and $25 / 75$ compositions and a single $T_{m}$ for the $50 / 50$ blend. A single $T_{m}$ is seen for the $C_{16} / C_{18}$ blends, with similar side chain lengths. Although the observation of a single $T_{m}$ for the $C_{16} / C_{18}$ blends would tend to imply miscibility, it is seen (Figure 3.1e) that the $T_{m}$ of the blends are not in-between those of $\mathrm{C}_{16}$ and $\mathrm{C}_{18}$ but lower than both of them, i.e., both are depressed and are significantly below the dotted (miscibility) line. This would indicate that the two biscarbamates are acting as mutual diluents ${ }^{16,17}$ (plasticizers) i.e., as eutectic mixtures and lowering each of the melting temperatures. The eutectic is seen with 50:50 composition. Likewise, the single $T_{m}$ seen for the $50 / 50$ blend of $C_{8} / C_{12}$ is lower than the $T_{m}$ of the $C_{8}$ 
and $\mathrm{C}_{12}$. Figure 3.1c for the $\mathrm{C}_{8} / \mathrm{C}_{12}$ melt blend shows the $\mathrm{T}_{\mathrm{m}}$ of the blends to be well below the dotted (miscibility) curve and the eutectic temperature for the $30 / 70$ composition. Xray diffraction discussed below shows that no co-crystallization occurred. In cases where two $\mathrm{T}_{\mathrm{m}}$ 's are seen, e.g., $\mathrm{C}_{4} / \mathrm{C}_{18}$ blends (Figure 3.1a), the first $\mathrm{T}_{\mathrm{m}}$ is lower than that of $\mathrm{C}_{4}$ and it decreases from 93.9 to 74.6 with an increase in $\mathrm{C}_{18}$. The second $\mathrm{T}_{\mathrm{m}}$ (which can be associated with the $\mathrm{C}_{18}$ component) is lower than that of pure $\mathrm{C}_{18}$, and it decreases from 121.9 to $104.8^{\circ} \mathrm{C}$ with an increase in $\mathrm{C}_{4}$ content. The corresponding curve shown in Figure 3.1a is close to the dotted line, which would lead to the conclusion that a fraction of the sample is miscible, and the rest acts as a eutectic mixture.

Table 3.2. Melting points of blends of biscarbamates prepared by solvent and melt mixing.

\begin{tabular}{lllllll} 
& \multicolumn{2}{c}{$\mathrm{C}_{4} / \mathrm{C}_{18}$ blend } & \multicolumn{3}{c}{$\mathrm{C}_{8} / \mathrm{C}_{12}$ blend } & \multicolumn{2}{c}{$\mathrm{C}_{16} / \mathrm{C}_{18}$ blend } \\
\cline { 2 - 7 } $\begin{array}{l}\text { Sample } \\
\text { ratio }\end{array}$ & $\mathrm{T}_{\mathrm{m}},{ }^{\circ} \mathrm{C}$ & $\mathrm{T}_{\mathrm{m}},{ }^{\circ} \mathrm{C}$ & $\mathrm{T}_{\mathrm{m}},{ }^{\circ} \mathrm{C}$ & $\mathrm{T}_{\mathrm{m}},{ }^{\circ} \mathrm{C}$ & $\mathrm{T}_{\mathrm{m}},{ }^{\circ} \mathrm{C}$ & $\mathrm{T}_{\mathrm{m}},{ }^{\circ} \mathrm{C}$ \\
& solv mix & melt blend & solv mix & melt blend & solv mix & melt blend \\
\hline $100 / 0$ & 93.9 & 93.9 & 108.4 & 108.4 & 118.8 & 118.8 \\
$75 / 25$ & $92.9,109.4$ & $87.3,104.8$ & 104.8 & $97.3,100.3$ & 117.9 & 115.6 \\
$50 / 50$ & $92.7,113.9$ & $81.7,109.2$ & 103.0 & 96.9 & 117.1 & 114.6 \\
$25 / 75$ & $92.1,117.9$ & $74.6,113.1$ & $102.5,110.2$ & $95.3,99.8$ & 119.1 & 116.9 \\
$0 / 100$ & 121.9 & 121.9 & 115.7 & 115.7 & 121.9 & 121.9 \\
\hline
\end{tabular}


(a)

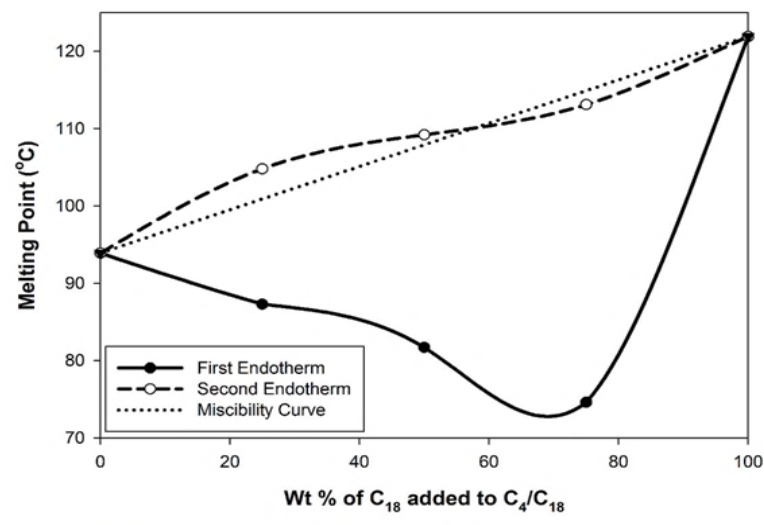

(c)

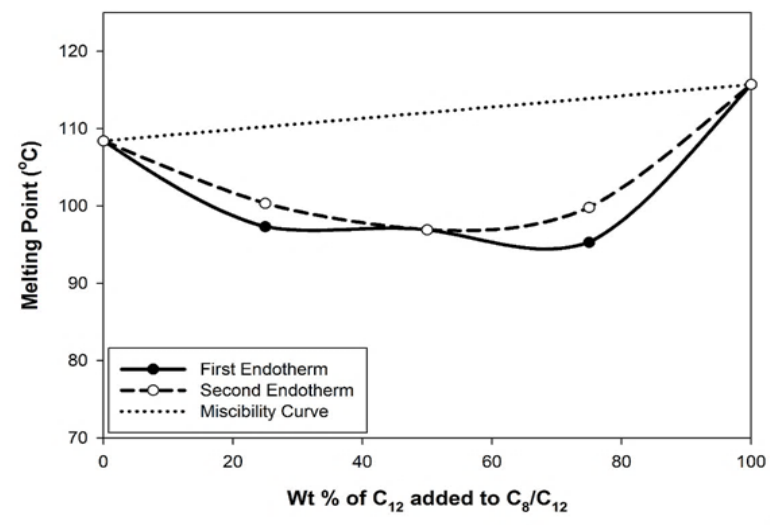

(e) (b) $\quad \mathrm{C}_{4} / \mathrm{C}_{18}$ Solvent-Based Blend

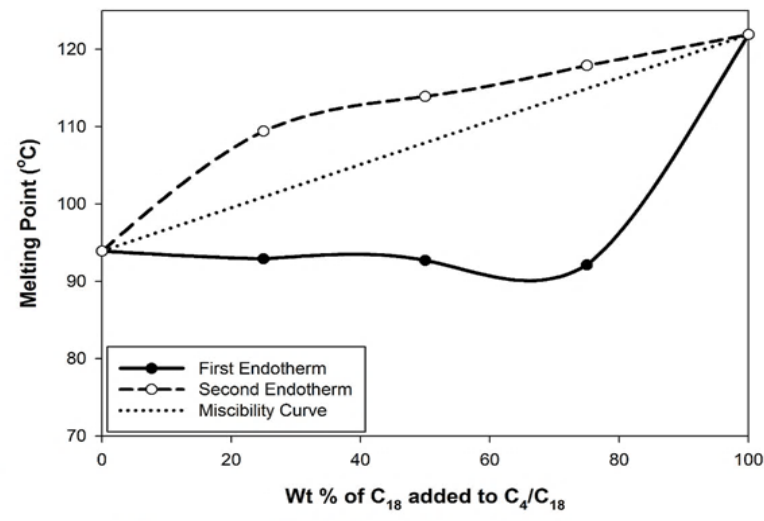

(d)

$\mathrm{C}_{8} / \mathrm{C}_{12}$ Solvent-Based Blend

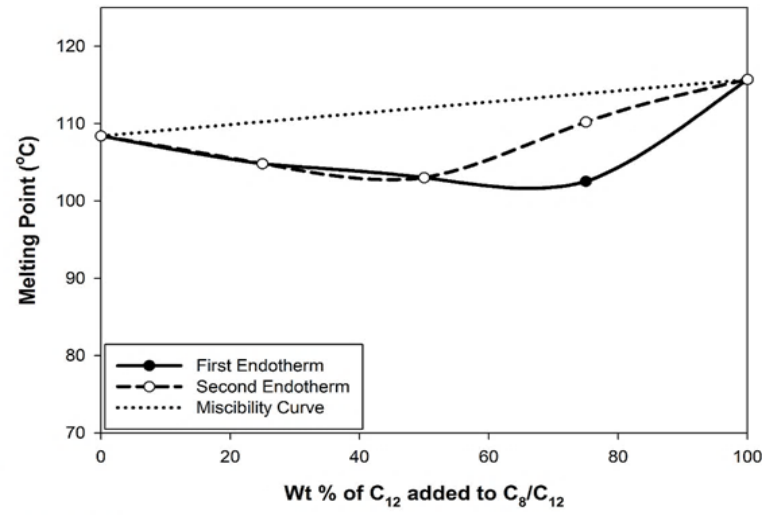

$\mathrm{C}_{16} / \mathrm{C}_{18}$ Blends

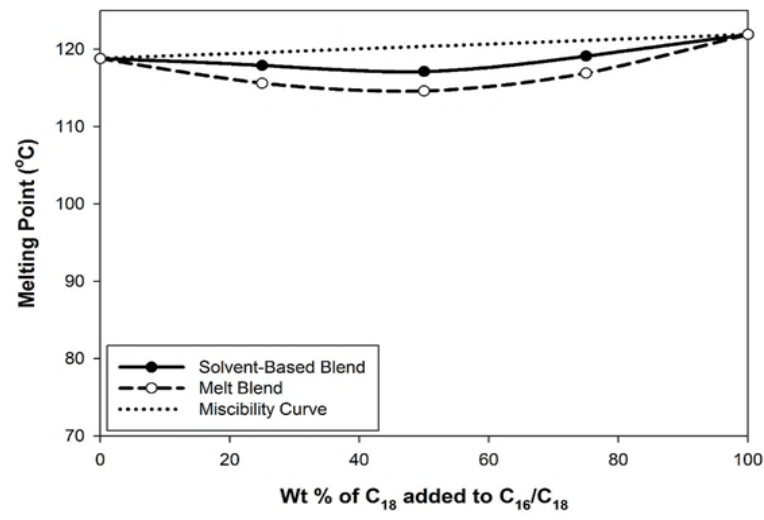

Figure 3.1. The melting temperatures $\left(T_{m}\right)$ at various compositions and the miscibility curves for $\mathrm{C}_{4} / \mathrm{C}_{18}$ (a) melt and (b) solvent blends, $\mathrm{C}_{8} / \mathrm{C}_{12}$ (c) melt and (d) solvent blends, and (e) $\mathrm{C}_{16} / \mathrm{C}_{18}$ melt and solvent blends. Both $\mathrm{C}_{4} / \mathrm{C}_{18}$ and $\mathrm{C}_{8} / \mathrm{C}_{12}$ blends have multiple melting points (endotherms). 
We discussed the influence of the length of the alkyl chain on the rates of crystallization and spherulite growth of these biscarbamates from the melt in a previous publication. ${ }^{12,13}$ Both rates showed a maximum with the $\mathrm{C}_{8}$ biscarbamates. The difference was significant between $\mathrm{C}_{4}, \mathrm{C}_{8}$ and $\mathrm{C}_{12}$, and that between $\mathrm{C}_{16}$ and $\mathrm{C}_{18}$ was small. The halftime ( $\left(\mathrm{t}_{1}\right)$ ) for completion of crystallization was 7.8 and 39 seconds, respectively, for $\mathrm{C}_{8}$ and $\mathrm{C}_{12}$. Such differences in the rate of crystallization from the melt could affect the mixing at the molecular level.

\subsection{Blends from Solution}

Since the differences in the rate of crystallization from the melt lead to self-sorting, we explored the behaviour of blends that were precipitated from solution. As described in the Experimental section, the solution based blends were prepared by heating $\left(70-80{ }^{\circ} \mathrm{C}\right)$ the components in chloroform and slowly cooling. The temperatures of dissolution and precipitation for the blends are listed in Table 3.3. It was observed that except for the $\mathrm{C}_{4} / \mathrm{C}_{18}$ blends, precipitation occurred at a single temperature although the individual components by themselves precipitated at different temperatures (Table 3.1). For the $\mathrm{C}_{8} / \mathrm{C}_{12}$ and $\mathrm{C}_{16} / \mathrm{C}_{18}$ blends with various relative concentrations, the precipitation temperatures were lower than that of the shorter side chain. For example, the precipitation temperatures of $\mathrm{C}_{16}$ and $\mathrm{C}_{18}$ are 39.0 and $49.2{ }^{\circ} \mathrm{C}$, respectively (Table 3.1 ). With the blends, the precipitation temperatures are lower, ranging from 32.8 to 25.8 (Table 3.3). In the case of $\mathrm{C}_{4} / \mathrm{C}_{18}$ blends for which two precipitation temperatures are seen, both lie between those of $\mathrm{C}_{4}$ and $\mathrm{C}_{18}$. Note that the difference in the precipitation temperatures of $\mathrm{C}_{4}$ and $\mathrm{C}_{18}$ is large. 
Table 3.3. Temperatures of dissolution and precipitation of various biscarbamates blends from chloroform. All the samples are prepared using the same amount of substrate and solvent.

\begin{tabular}{|c|c|c|c|}
\hline & Concentration & $\mathrm{T}_{\text {dissolution }}\left({ }^{\circ} \mathrm{C}\right)$ & $\mathrm{T}_{\text {precipitation }}\left({ }^{\circ} \mathrm{C}\right)$ \\
\hline \multirow[t]{5}{*}{$\mathrm{C}_{16} / \mathrm{C}_{18}$} & $0 / 100$ & 55.5 & 49.2 \\
\hline & $25 / 75$ & 53.0 & 32.8 \\
\hline & $50 / 50$ & 52.5 & 28.0 \\
\hline & $75 / 25$ & 50.2 & 25.8 \\
\hline & $100 / 0$ & 50.8 & 39.0 \\
\hline \multirow[t]{5}{*}{$\mathrm{C}_{8} / \mathrm{C}_{12}$} & $0 / 100$ & 42.0 & 17.0 \\
\hline & $25 / 75$ & 38.6 & 4.2 \\
\hline & $50 / 50$ & 38.0 & 3.8 \\
\hline & $75 / 25$ & 34.5 & 1.3 \\
\hline & $100 / 0$ & 23.8 & 7.0 \\
\hline \multirow[t]{5}{*}{$\mathrm{C}_{4} / \mathrm{C}_{18}$} & $0 / 100$ & 55.5 & 49.2 \\
\hline & $25 / 75$ & 54.5 & 38.5 and 1.7 \\
\hline & $50 / 50$ & 53.5 & 34.6 and 1.1 \\
\hline & $75 / 25$ & 48.2 & 30.1 and 0.6 \\
\hline & $100 / 0$ & 17.5 & -8.5 \\
\hline
\end{tabular}

The melting temperatures of the solution based blends are given in Table 3.2. In this case again, a melting temperature intermediate between those of the pure components is not seen. Two $\mathrm{T}_{\mathrm{m}}$ 's are observed for $\mathrm{C}_{4} / \mathrm{C}_{18}$ with all blend compositions, and for the 
25/75 composition for the $C_{8} / C_{12}$ blend. As in the case of melt-mixed blends, the first $T_{m}$ is lower than that of the first member of the blend and the second $\mathrm{T}_{\mathrm{m}}$ lower than that of the second member, indicating mutual dilution resulting in depression of the $\mathrm{T}_{\mathrm{m}}$ 's. Table 3.2 also shows that the extent of depression of the $T_{m}$ 's $\left(\Delta T_{m}\right)$ of the solution based blends (from those of the pure components) is smaller than in the case of melt-mixed blends. Figure 3.2 shows the plots of the change in melting temperatures $\left(\Delta \mathrm{T}_{\mathrm{m}}\right)$ of component $\mathrm{A}$ as a function of the concentration of component $\mathrm{B}$ in the $\mathrm{A} / \mathrm{B}$ blends. Figure 3.2 (a) and (b) correspond to the $\mathrm{C}_{4} / \mathrm{C}_{18}$ blend, with (a) showing the $\Delta \mathrm{T}_{\mathrm{m}}$ of $\mathrm{C}_{4}$ with $\mathrm{C}_{18}$ concentration and (b) showing the effect of $\mathrm{C}_{4}$ on the $\Delta \mathrm{T}_{\mathrm{m}}$ of $\mathrm{C}_{18}$. It is seen that with all the blends, the $\Delta \mathrm{T}_{\mathrm{m}}$ (depression of $\mathrm{T}_{\mathrm{m}}$ due to mutual dilution) resulting from solution blending is less than that from melt-mixing. Figure 3.2 also shows that in the case of solution based blends, the biscarbamates with a shorter side chain causes a higher $\Delta \mathrm{T}_{\mathrm{m}}$ for the longer alkyl chain component than vice versa. This can be attributed to the higher rate of diffusion of the smaller molecule into the domains of the larger. 

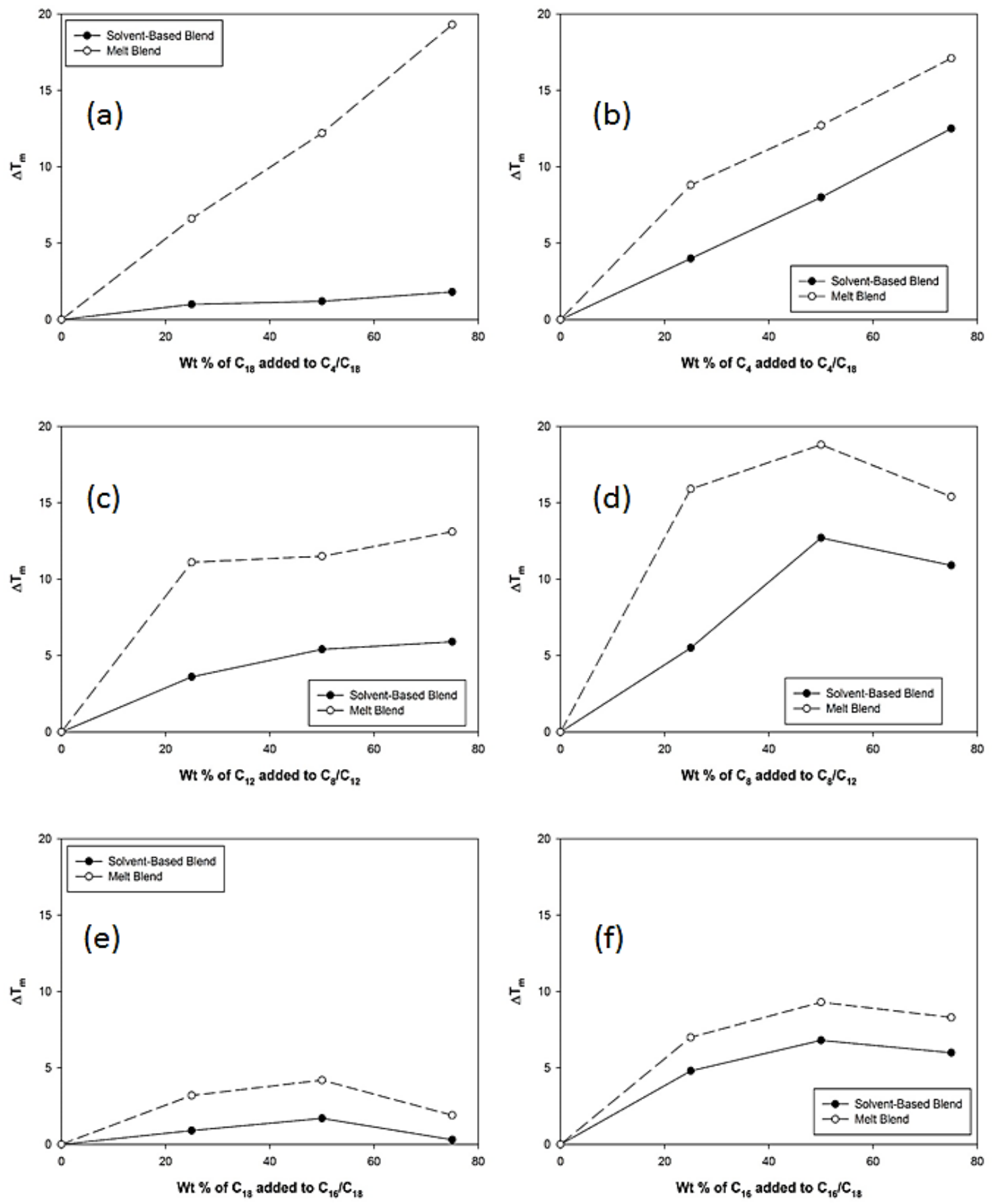

Figure 3.2. Variation of $\Delta T_{m}$ for (a) $C_{4}$ and (b) $\mathrm{C}_{18}$ for the $\mathrm{C}_{4} / \mathrm{C}_{18}$ blend, (c) $\mathrm{C}_{8}$ and (d) $\mathrm{C}_{12}$ for the $\mathrm{C}_{8} / \mathrm{C}_{12}$ blend, and (e) $\mathrm{C}_{16}$ and (f) $\mathrm{C}_{18}$ for the $\mathrm{C}_{16} / \mathrm{C}_{18}$ blend with addition of the other component. There is a larger change in $\Delta \mathrm{T}_{\mathrm{m}}$ for the melt blends compared to the solventbased blends. 
Figure 3.3 shows the DSC traces of the solution based $\mathrm{C}_{8} / \mathrm{C}_{12}$ blends for various compositions. Although we listed a single melting temperature in Table 3.2 for the $75 / 25$ blend, Figure $3.3 \mathrm{~b}$ shows that the endotherm is not a discrete peak but a shoulder appears at a lower temperature. Likewise, a shoulder at the higher temperature is seen in Figure 3.3e for the $40 / 60$ blend. Single melting endotherm is seen only for the $60 / 40$ and $50 / 50$ blends. Although the $\Delta \mathrm{T}_{\mathrm{m}}$ for the solution based blends were smaller than those of the meltmixed, upon heating these solution based samples to about $20{ }^{\circ} \mathrm{C}$ above the melting temperature and recording the DSC traces again showed that the resulting $\mathrm{T}_{\mathrm{m}}$ 's were similar to those of the melt-mixed blends. Thus, when the melt is cooled, the differences in the rate of crystallization leads to self-sorting (although there is a dilution effect), while the common precipitation temperature in the case of the solution leads to less of a mutualdilution effect, although self-sorting is still present. 

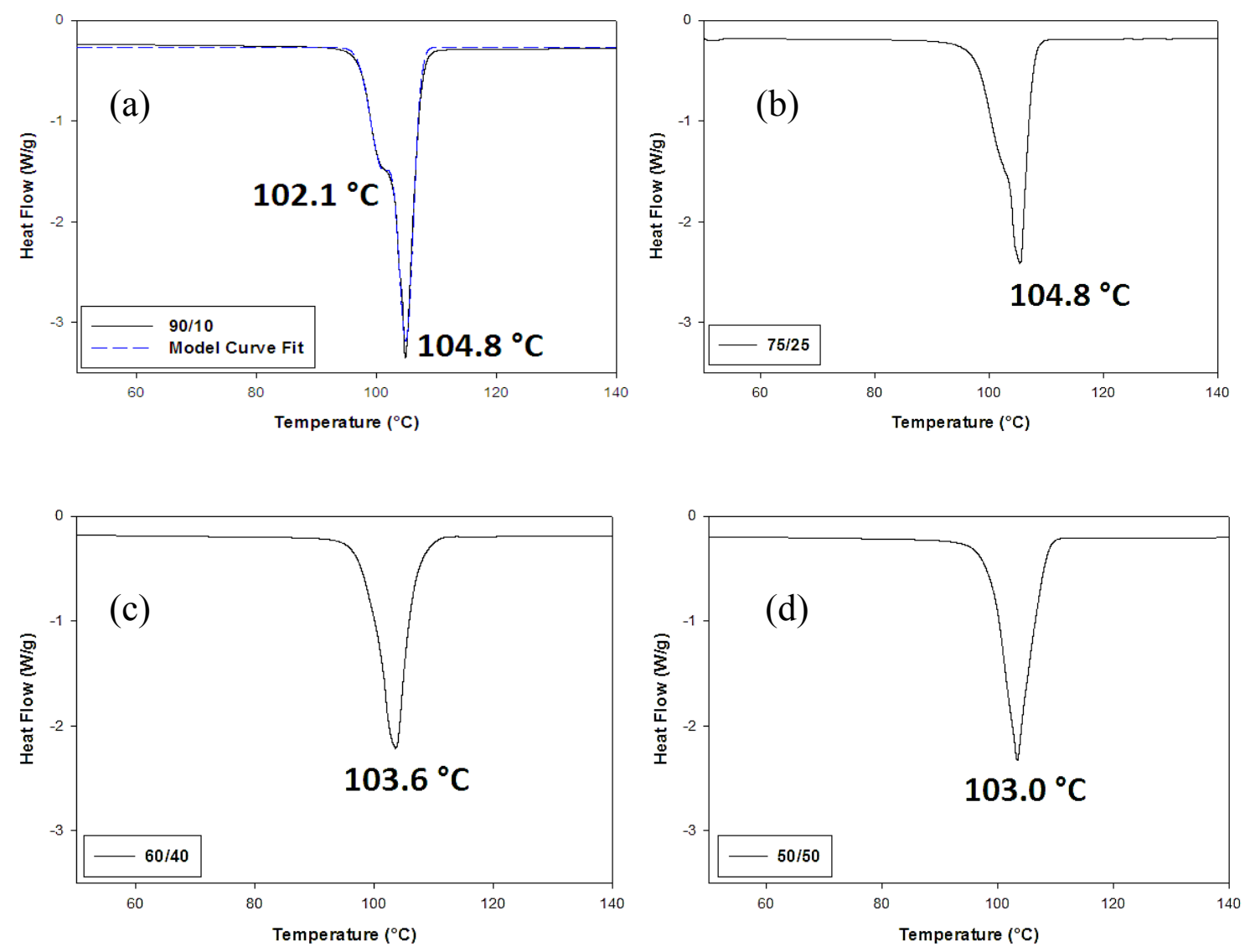

(Figure 3.3 cont'd on next page) 

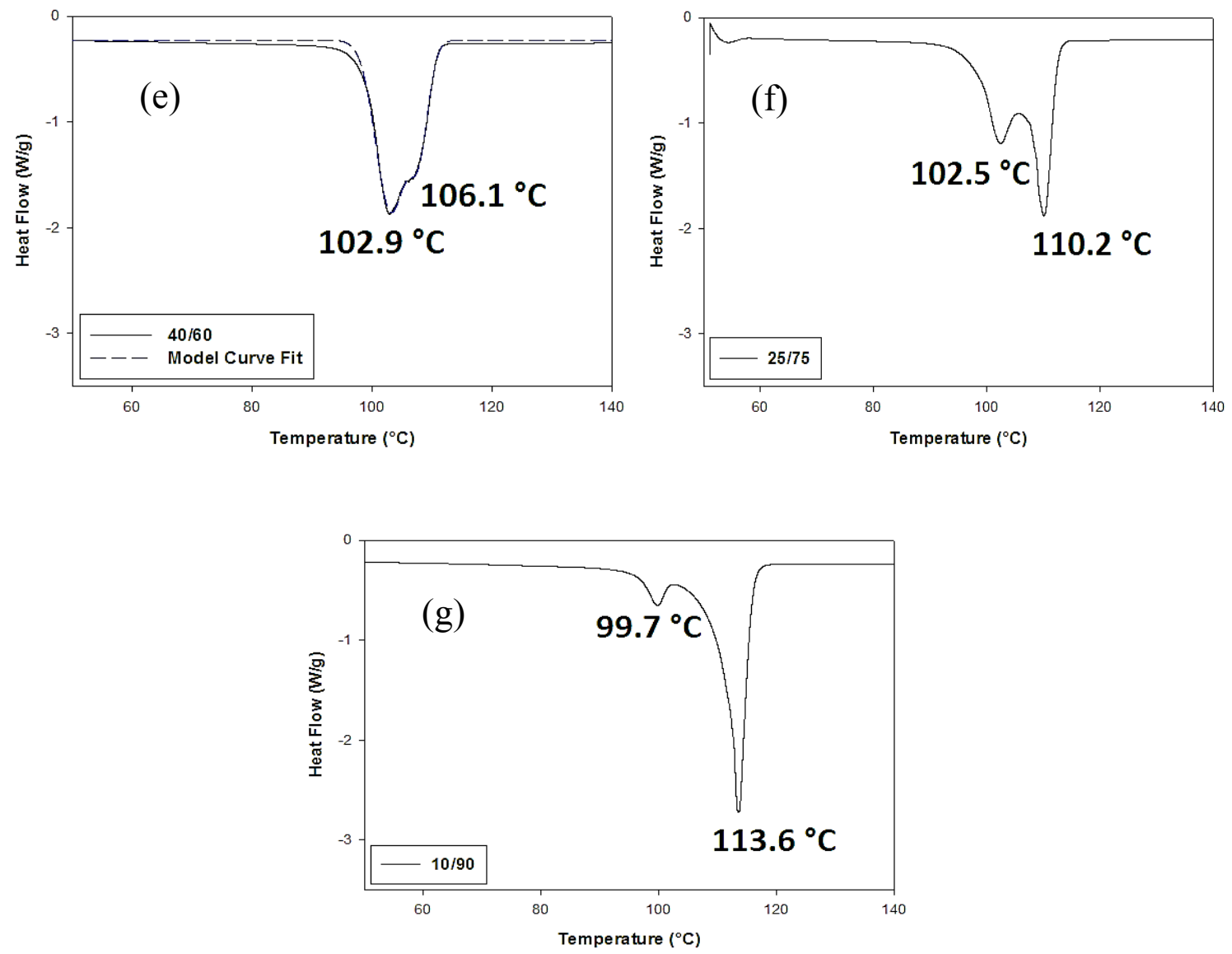

Figure 3.3. $D S C$ traces of $C_{8} / C_{12}$ biscarmabates blends at various concentrations: (a) $90 / 10$, (b) 75/25, (c) 60/40, (d) 50/50, (e) 40/60, (f) 25/75, and (g) 10/90. The peaks become narrower as the relative concentrations of the components become comparable. In the cases of (a), (b) and (e), TA Universal Analysis software could not identify the location of the shoulder peak. The values of the peaks were then obtained using a model fit by Igor Pro.

If self-sorting is suppressed (i.e., mixing at the molecular level and cocrystallization occurs), it would be reflected in the X-ray diffraction pattern as well. However, if only the domains are intermixed as shown schematically in Figure 3.4, no 
change in the diffraction would be observed, although the $\mathrm{T}_{\mathrm{m}}$ would change due to plasticization. We discussed before that the longest d-spacing in the X-ray diffraction of the biscarbamates increases linearly with the length of the alkyl chain. ${ }^{12}$ The IR spectra showed that all the $\mathrm{N}-\mathrm{H}$ groups are hydrogen bonded to the $\mathrm{C}=\mathrm{O}$ bonds. X-ray diffraction from oriented lamellae of the spherulites showed that distance between molecules in the hydrogen bonded plane is about $4.6 \AA$ (similar to the distance between the chains in polyethylene) and that the distance between these planes is about 3.6-3.8 $\AA$. In the case of co-crystallization, any change in the d-spacings would occur along the direction corresponding to the length of the molecules and not in the spacings (4.6 and $3.6 \AA$ ) corresponding to the hydrogen bond planes. Table 3.4 shows the list of d-spacings recorded for the solution based $\mathrm{C}_{8} / \mathrm{C}_{12}$ blends. The reflections at 3.6 and $4.4 \AA$ are present in all the blends. The longest spacing recorded for $\mathrm{C}_{12}$ and $\mathrm{C}_{8}$ are 20.8 and $18.2 \AA$ respectively. These reflections appear separately for all the blend compositions. Similar is the case with other reflections, i.e., the diffraction pattern is an overlap of those of the individual components and do not show changes that would indicate co-crystallization. Figure 3.5 shows the change in crystallinity as a function of the composition of the $\mathrm{C}_{8} / \mathrm{C}_{12}$ blend. It is seen that a minimum in crystallinity is seen with 50/50 composition, similar to that in the melt-mixed blends. 
Assembly of Molecule A

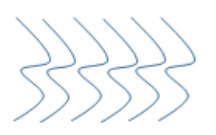

Intercalation of $A$ and $B$ at the molecular level 3513535
Assembly of Molecule B

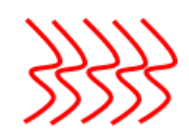

Mixture of domains of $A$ and $B$

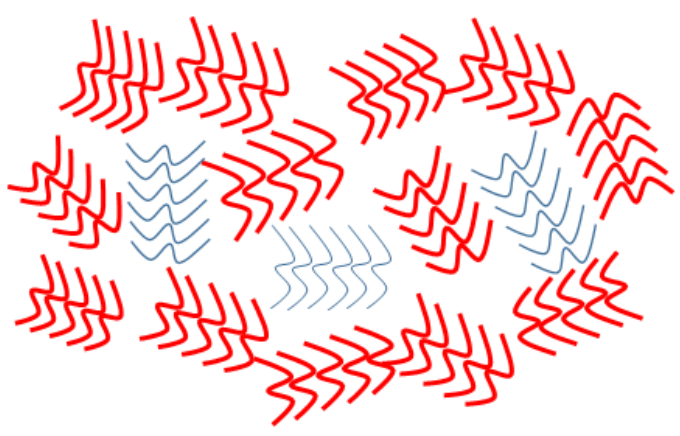

Figure 3.4. Model of domain mix versus molecular mix. 
Table 3.4. Bragg d-spacings of the peaks and the corresponding intensities (I(\%)) normalized with respect to the peak at $\mathrm{d}=3.6 \AA$ for $\mathrm{C}_{8} / \mathrm{C}_{12}$ solvent-based blend samples of various relative concentrations.

\begin{tabular}{|c|c|c|c|c|c|c|c|c|c|}
\hline \multicolumn{2}{|c|}{$0 / 100$} & \multicolumn{2}{|c|}{$25 / 75$} & \multicolumn{2}{|c|}{$50 / 50$} & \multicolumn{2}{|c|}{$75 / 25$} & \multicolumn{2}{|c|}{$100 / 0$} \\
\hline $\mathrm{d}(\AA)$ & I (\%) & $\mathrm{d}(\AA)$ & I (\%) & $\mathrm{d}(\AA)$ & I (\%) & $\mathrm{d}(\AA)$ & I (\%) & d $(\AA)$ & I (\%) \\
\hline 20.8 & 369.1 & 21.0 & 251.6 & 20.6 & 363.3 & 20.7 & 446.3 & & \\
\hline & & 18.2 & 98.0 & 17.8 & 316.0 & 17.8 & 508.3 & 18.2 & 481.3 \\
\hline 16.6 & 25.4 & $*$ & & $*$ & & & & & \\
\hline 12.9 & 8.1 & 12.9 & 6.9 & 12.9 & 5.5 & & & & \\
\hline & & 10.7 & 6.4 & 10.6 & 19.9 & 10.6 & 20.0 & 10.8 & 38.0 \\
\hline 7.3 & 53.0 & 7.2 & 42.8 & 7.2 & 23.3 & 7.2 & 13.8 & 7.7 & 9.4 \\
\hline 6.0 & 5.6 & 5.9 & 12.3 & 5.9 & 25.6 & 5.9 & 27.8 & 5.9 & 47.9 \\
\hline 5.1 & 13.2 & 5.0 & 10.1 & 5.0 & 5.2 & & & & \\
\hline 4.4 & 5.0 & 4.3 & 5.8 & 4.3 & 7.8 & 4.3 & 25.0 & 4.3 & 27.8 \\
\hline 4.2 & 4.1 & 4.2 & 5.2 & 4.2 & 6.1 & 4.1 & 18.5 & 4.1 & 18.8 \\
\hline 3.8 & 8.1 & 3.8 & 10.8 & 3.8 & 11.7 & 3.8 & 34.8 & 3.9 & 37.0 \\
\hline 3.6 & 100.0 & 3.5 & 100.0 & 3.5 & 100.0 & 3.5 & 100.0 & 3.6 & 100.0 \\
\hline & & & & 3.4 & 8.7 & 3.4 & 20.5 & 3.4 & 28.1 \\
\hline 3.3 & 38.7 & 3.3 & 35.2 & 3.3 & 25.9 & 3.2 & 23.0 & 3.3 & 33.0 \\
\hline
\end{tabular}

* Cannot detect peak as it is hidden in the slope of another peak. 


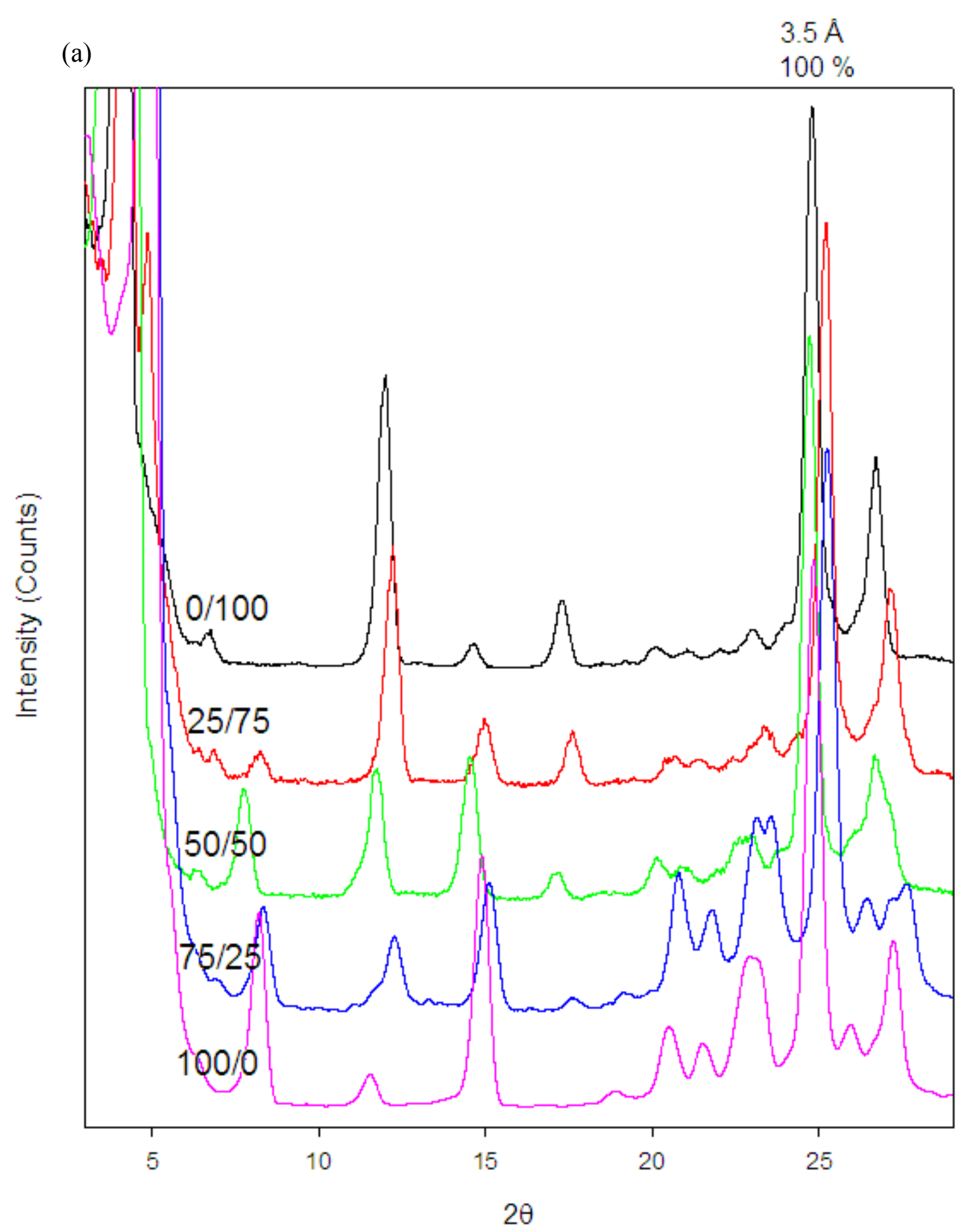

(Figure 3.5 cont'd on next page) 


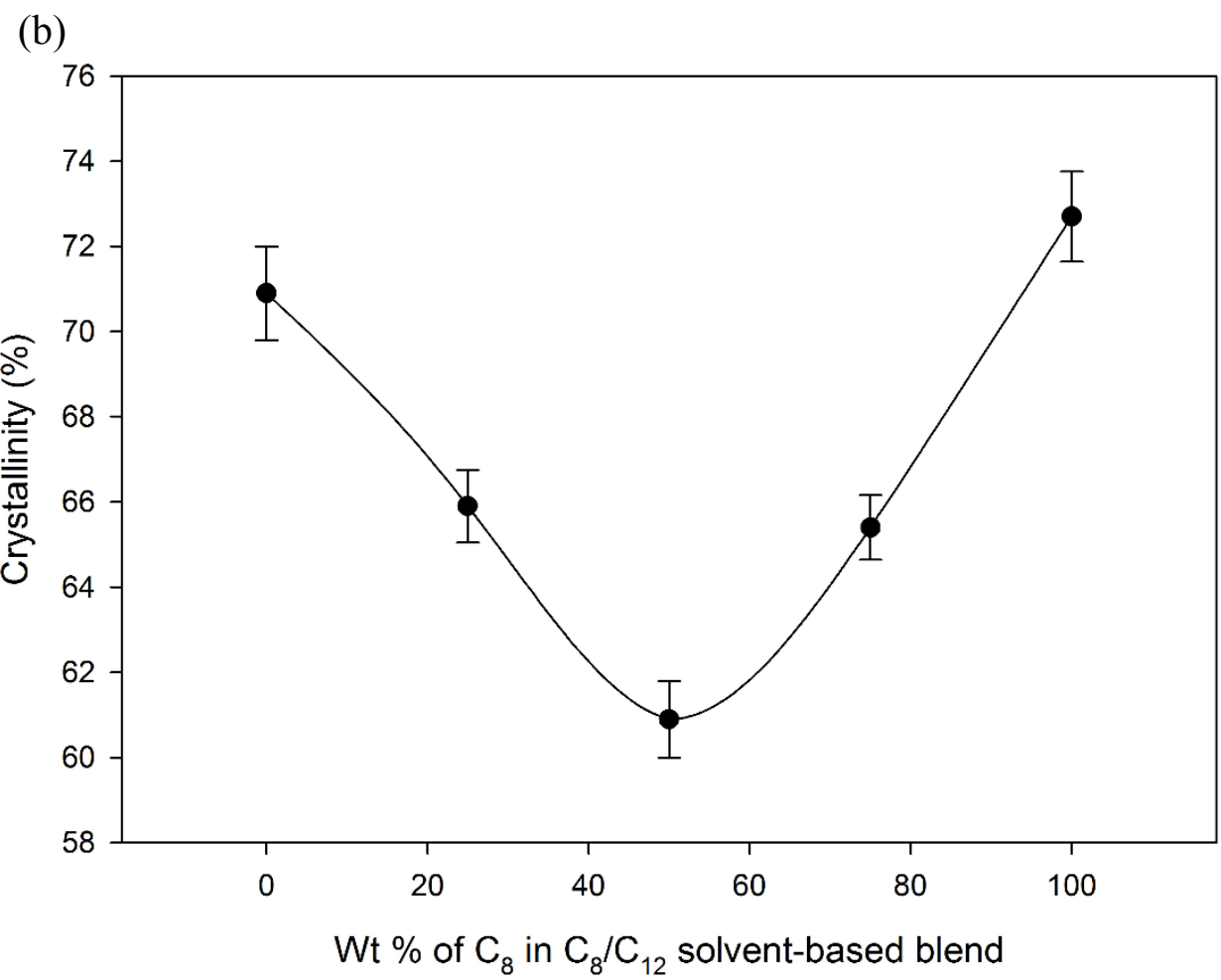

Figure 3.5. (a) Normalized XRD patterns of $\mathrm{C}_{8} / \mathrm{C}_{12}$ biscarbamate blends obtained from solvent mixing at various concentrations. The peaks are in the same positions, but the widths and height differ. This is an indication of the change in crystallinity of the blends. (b) Percent crystallinity of $\mathrm{C}_{8} / \mathrm{C}_{12}$ solvent-based blend showing a change in crystallinity as a function of concentration.

Figure 3.6 shows the optical micrographs of the $\mathrm{C}_{8} / \mathrm{C}_{12}$ blends recorded at select temperatures. It is seen in Figure 3.3 that the $\mathrm{C}_{8} / \mathrm{C}_{12}: 10 / 90$ blend shows two endotherms, at 99.7 and $113.6^{\circ} \mathrm{C}$. The $\mathrm{OM}$ on Figure 3.6a shows that upon heating this blend to 100 ${ }^{\circ} \mathrm{C}$, the $\mathrm{C}_{8}$ melted and the aggregates of crystals of $\mathrm{C}_{12}$ are left over. The latter melted only at $114{ }^{\circ} \mathrm{C}$. Thus, the components existed as individual domains, and not in a co-crystallized 
form, as indicated by $\mathrm{X}$-ray diffraction. Figure $3.3 \mathrm{~d}$ shows a single endotherm at $103{ }^{\circ} \mathrm{C}$ for the $\mathrm{C}_{8} / \mathrm{C}_{12}: 50 / 50$ blend. Accordingly, the $\mathrm{OM}$ in Figure 3.6b shows that the entire blend sample melted at $103{ }^{\circ} \mathrm{C}$ due to mutual diluent effect, while X-ray diffraction showed no co-crystallization.

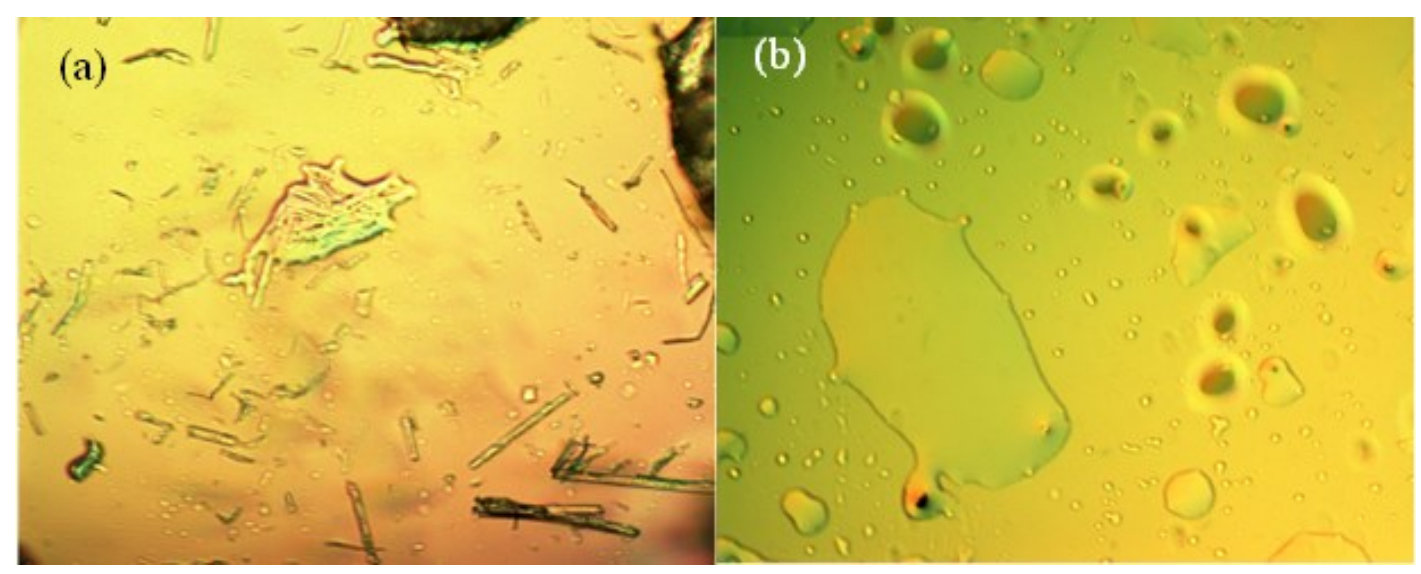

Figure 3.6. Optical micrographs of (a) $\mathrm{C}_{8} / \mathrm{C}_{12}: 10 / 90$ at $100{ }^{\circ} \mathrm{C}$ and (b) $\mathrm{C}_{8} / \mathrm{C}_{12}: 50 / 50$ at 103 ${ }^{\circ} \mathrm{C}$.

As discussed above, the melting temperatures are affected in these blends due to mutual plasticization. In the case of melt-mixed blends, our previous study on $\mathrm{C}_{8} / \mathrm{C}_{12}$ blend showed that the growth rate of spherulites was reduced by mixing and that it showed a minimum at 50:50 composition. Since we investigated precipitation from solution as a means to suppress self-sorting, we measured the rate of growth of spherulites upon precipitation from solution. To this end, a drop of hot biscarbamate solution in chloroform was placed in a concave microscope slide, the area around the solvent was sealed and a 
cover slidevwas placed over it. Images were recorded as the spherulites appeared when the temperature of the system was kept at $5^{\circ} \mathrm{C}$, as it is the temperature at which the biscarbamate blends precipitates out of chloroform (Table 3.3). The precipitates were not in the form of single crystals, but lamellae of spherulites. OM images of the $\mathrm{C}_{8} / \mathrm{C}_{12}$ biscarbamate precipitation were taken using an optical microscope at a rate of approximately 0.85 second interval (Figures 3.7 and 3.8 ). In contrast to the impinging spherulites grown from the melt blends, those from the solvent-based blend are packed less densely leaving more voids when the biscarbamates precipitate out (Figure 3.8). This is due to the fewer nucleation sites, leading to more spreading and larger spherulites. ${ }^{18}$ 


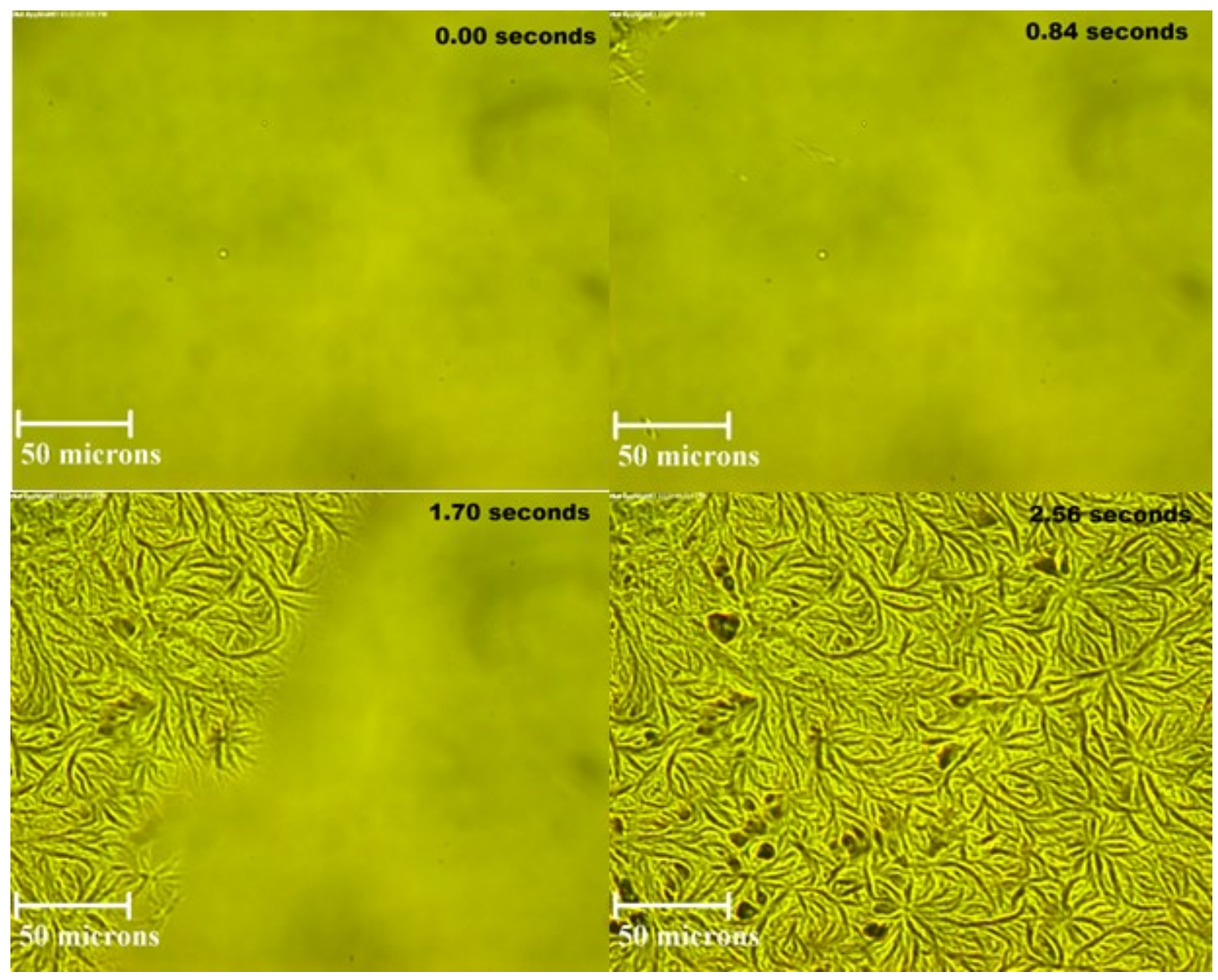

Figure 3.7. Optical micrographs of biscarbamate $\mathrm{C}_{8} / \mathrm{C}_{12}(50 / 50)$ prepared by solventmixing taken various time frames during spherulite growth. 


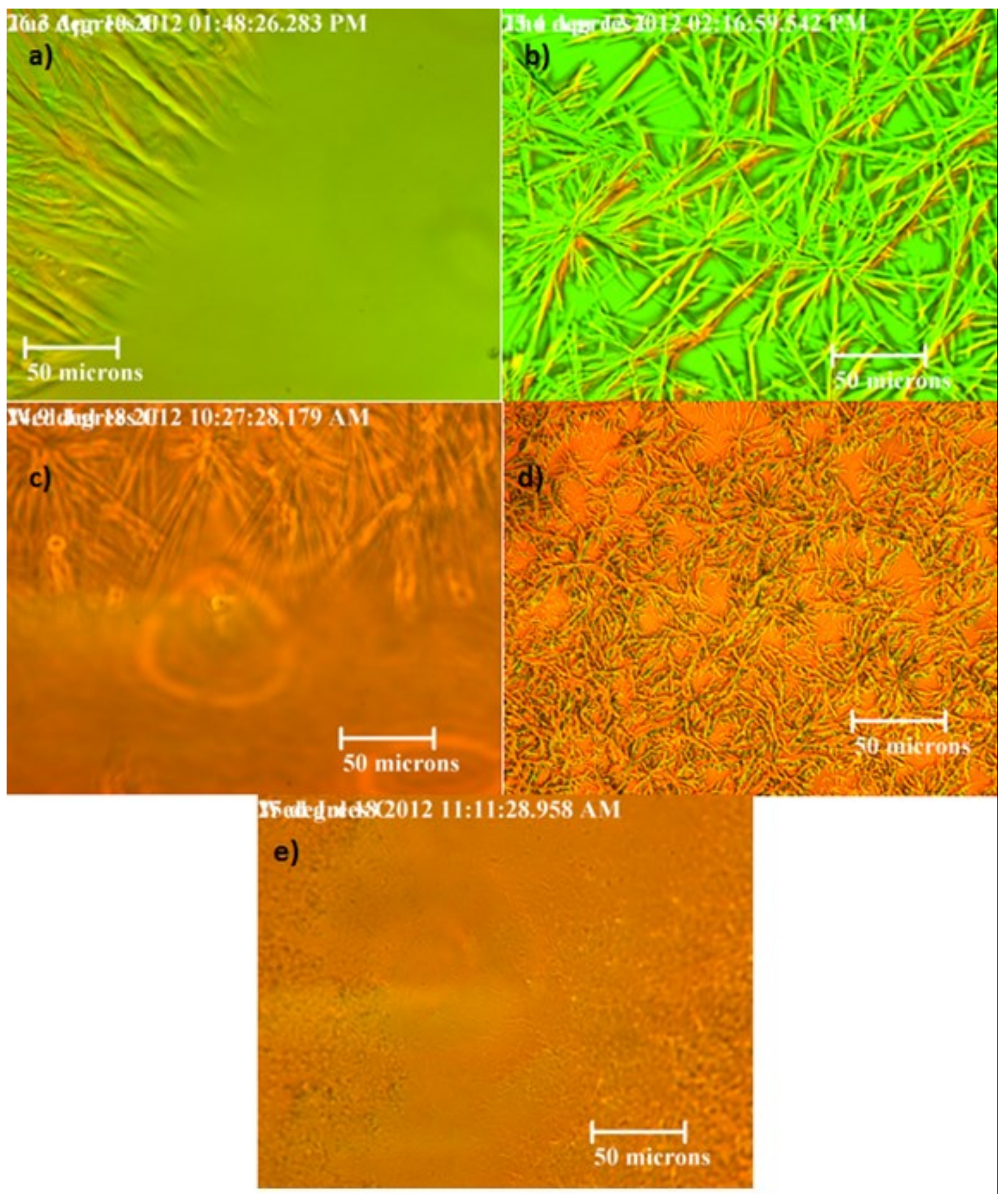

Figure 3.8. Optical micrographs of individual biscarbamates $\mathrm{C}_{8}(\mathrm{a})$ and $\mathrm{C}_{12}(\mathrm{~b})$, and blends at concentration $25 / 75$ (c), 50/50 (d) and 75/25 (e) crystallized from the solvent as the temperature is kept at $5^{\circ} \mathrm{C}$. 
The increase in the size of the spherulites of the $\mathrm{C}_{8} / \mathrm{C}_{12}$ blends with time is shown in Figure 3.9a. The times required for the completion of the growth are about $6,8,13,8$, and 7 seconds for the $100 / 0,75 / 25,50 / 50,25 / 75$ and $0 / 100$ blends, respectively. The growth rates of the $100 / 0,75 / 25,50 / 50,25 / 75$ and $0 / 100$ blends from chloroform are about $34.3,17.8,9.6,14.5$ and $21.6 \mu \mathrm{m} / \mathrm{s}$ respectively. Figure $3.9 \mathrm{~b}$ shows the decrease in the growth rates of the blends, with a minimum at $\mathrm{C}_{8} / \mathrm{C}_{12}: 50 / 50$. This is similar to the change in crystallinity of these (solution) blends as well as for the melt-mixed blends. Significant differences are noted in the growth rates of solution based and melt-mixed blends (Figure 9 of Ref 1): (1) the sizes of the melt-grown spherulites of $C_{8}$ and $C_{12}$ were larger than 1000 $\mu \mathrm{m}$ upon completion of growth. In the case of solution grown spherulites, these are less than $250 \mu \mathrm{m}$. It is realized that the spherulites size would depend on the under-cooling. In the case of melt-mixed blends, the isothermal crystallization was studied, at the crystallization temperature. In the current solution based blends, the precipitation temperature was used to measure the spherulites size and rate; (2) the growth rates for meltcrystallized $C_{8}$ and $C_{12}$ were 130 and $80 \mu \mathrm{m} / \mathrm{s}$ respectively. These are far less, with 34.3 and $21.6 \mu \mathrm{m} / \mathrm{s}$, respectively for the crystallization from solution; (3) the extent of reduction of growth rate for the blends is also significantly less for the solution based crystallization. With melt-mixed blends, the growth rate decreased from 130 and $80 \mu \mathrm{m} / \mathrm{s}$ for the $\mathrm{C}_{8}$ and $\mathrm{C}_{12}$ respectively, to $10 \mu \mathrm{m} / \mathrm{s}$ for the $50 / 50$ blend. In the case of solution based blends, the growth rate was $10 \mu \mathrm{m} / \mathrm{s}$, which is only about $25 \mu \mathrm{m} / \mathrm{s}$ less than that of $\mathrm{C}_{8}$ and $10 \mu \mathrm{m} / \mathrm{s}$ less than that of $\mathrm{C}_{12}$. Thus solution-precipitation showed significant differences in the crystallization characteristics of the blends in comparison with those prepared by meltmixing. 

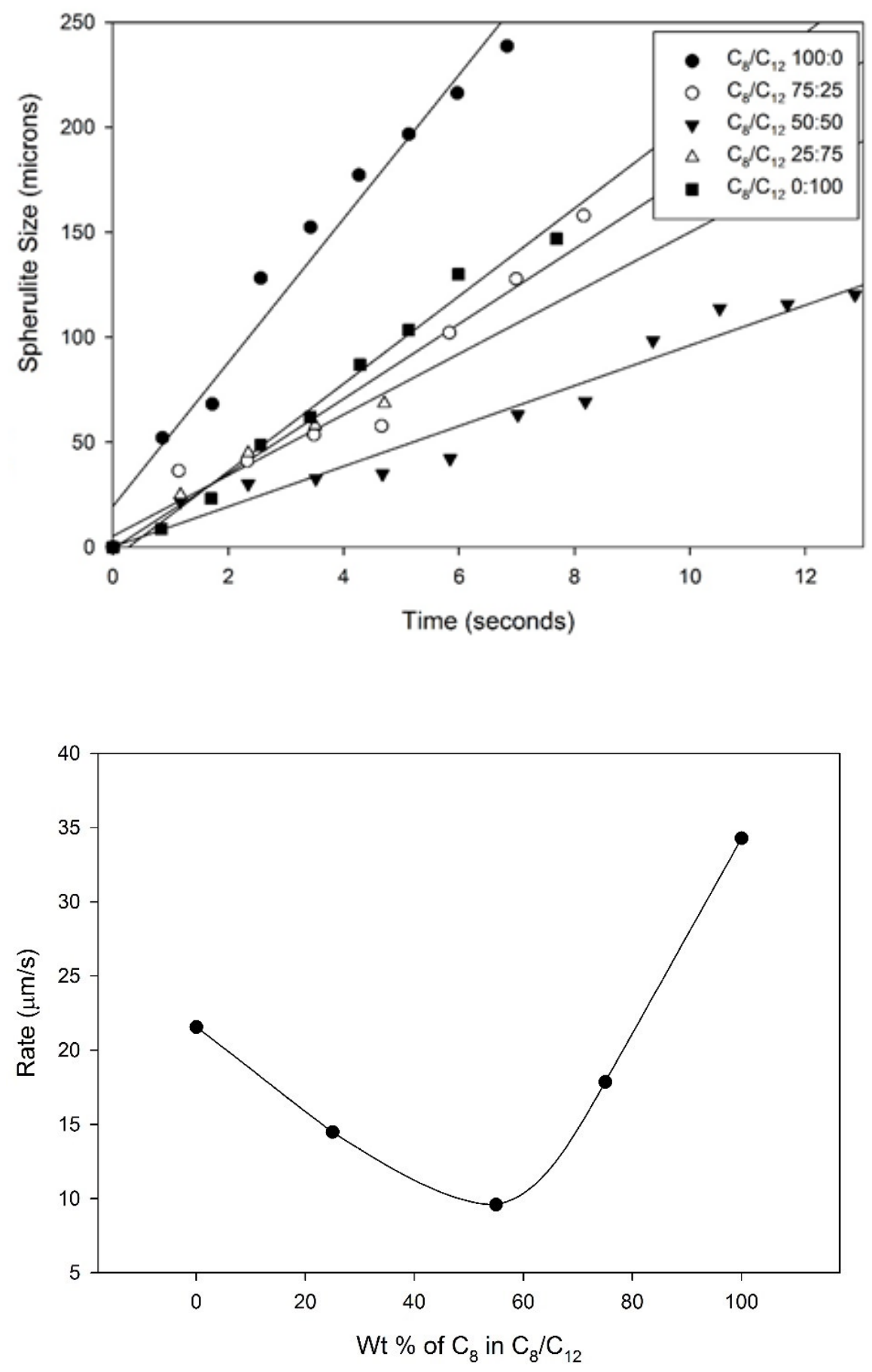

Figure 3.9. (top) Variation of spherulite size with time for $\mathrm{C}_{8}, \mathrm{C}_{12}$ and their blends when crystallized from solution (average of three experiments) and (bottom) the rate of spherulite growth as a function of wt $\% \mathrm{C}_{8}$ added to the $\mathrm{C}_{8} / \mathrm{C}_{12}$ solution blend. 


\subsection{Solvent Vapor and Thermal Annealing}

In the results presented above, even when a single melting endotherm is seen, the $T_{m}$ is not between those of the individual components, and the change in $T_{m}$ is due to the dilution effect. Attempts were made in the following sections to examine the possibility of encouraging molecular mixing which would indicate suppression of self-sorting. Solvent

vapor $^{19-22}$ or thermal annealing ${ }^{23-26}$ has been used in the past for driving a system towards equilibrium, and enhance mixing in the case of blends. The solution-mixed blends of $\mathrm{C}_{8} / \mathrm{C}_{12}$, with $25 / 75,50 / 50$ and $75 / 25$ (wt) ratios were exposed to chloroform vapor for up to six hours at room temperature and dried overnight in vacuum. The $\mathrm{T}_{\mathrm{m}}$ 's and the width of the endotherms at half-maximum (FWHM) are shown in Table 3.5. It is seen that there is no significant change in the melting temperatures and that there are still two endotherms for the $25 / 75$ blend. The FWHM shows a small increase indicating that there is some disorder upon vapor exposure.

Thermal annealing was performed on the solution-mixed $\mathrm{C}_{8} / \mathrm{C}_{12}$, by keeping the sample at $110^{\circ} \mathrm{C}$ (which is $2{ }^{\circ} \mathrm{C}$ above the $\mathrm{T}_{\mathrm{m}}$ of $\mathrm{C}_{8}$ ) for 20 minutes and then slowly cooling it to room temperature. The rationale is that the melt of $\mathrm{C}_{8}$ (with a melt viscosity of $10 \mathrm{cps}$ ) could diffuse into the crystalline domains of $\mathrm{C}_{12}$ and yield a molecular mixture. However, it did not happen, and the components self-sorted upon cooling. Figure 3.10 shows the DSC traces of the thermally annealed $\mathrm{C}_{8} / \mathrm{C}_{12}$ blends. Similar to the results shown in Table 3.2 for the melt-blends, two endotherms are seen for the $25 / 75$ and $75 / 25$ compositions, and one with a shoulder for the $50 / 50$ blend. The $\mathrm{T}_{\mathrm{m}}$ 's are lower than the individual components, showing the plasticization effect. 
Table 3.5. Melting temperatures and the full-width-half-max (FWHM) of $\mathrm{C}_{8} / \mathrm{C}_{12}$ blends of biscarbamates prepared by solvent mixing and processed through solvent annealing in chloroform at room temperature.

\begin{tabular}{|c|c|c|c|c|c|c|}
\hline & \multicolumn{2}{|c|}{$25 / 75$} & \multicolumn{2}{|c|}{$50 / 50$} & \multicolumn{2}{|c|}{$75 / 25$} \\
\hline Time, Hour & $\mathrm{T}_{\mathrm{m}},{ }^{\circ} \mathrm{C}$ & FWHM & $\mathrm{T}_{\mathrm{m}},{ }^{\circ} \mathrm{C}$ & FWHM & $\mathrm{T}_{\mathrm{m}},{ }^{\circ} \mathrm{C}$ & FWHM \\
\hline 0 & $101.8,110.4$ & $3.6,2.7$ & 103.4 & 4.9 & 104.8 & 3.4 \\
\hline 1 & $99.9,113.9$ & $3.2,2.8$ & $104.3,106.2$ & NA* & 103.4 & 4.7 \\
\hline 2 & $100.2,113.5$ & $3.9,3.2$ & 106.7 & 5.4 & 103.7 & 5.0 \\
\hline 3 & $100.2,113.3$ & $4.6,3.6$ & 104.7 & 5.8 & 102.9 & 4.1 \\
\hline 4 & $100.5,113.8$ & $5.1,3.0$ & 104.0 & 8.7 & 104.2 & 4.8 \\
\hline 5 & $100.4,111.9$ & $4.5,3.6$ & 105.2 & 7.5 & 103.3 & 4.7 \\
\hline 6 & $99.6,111.8$ & $5.5,2.9$ & 105.0 & 6.1 & 103.3 & 4.9 \\
\hline
\end{tabular}

* Data is unavailable due to baseline alignment issues. 


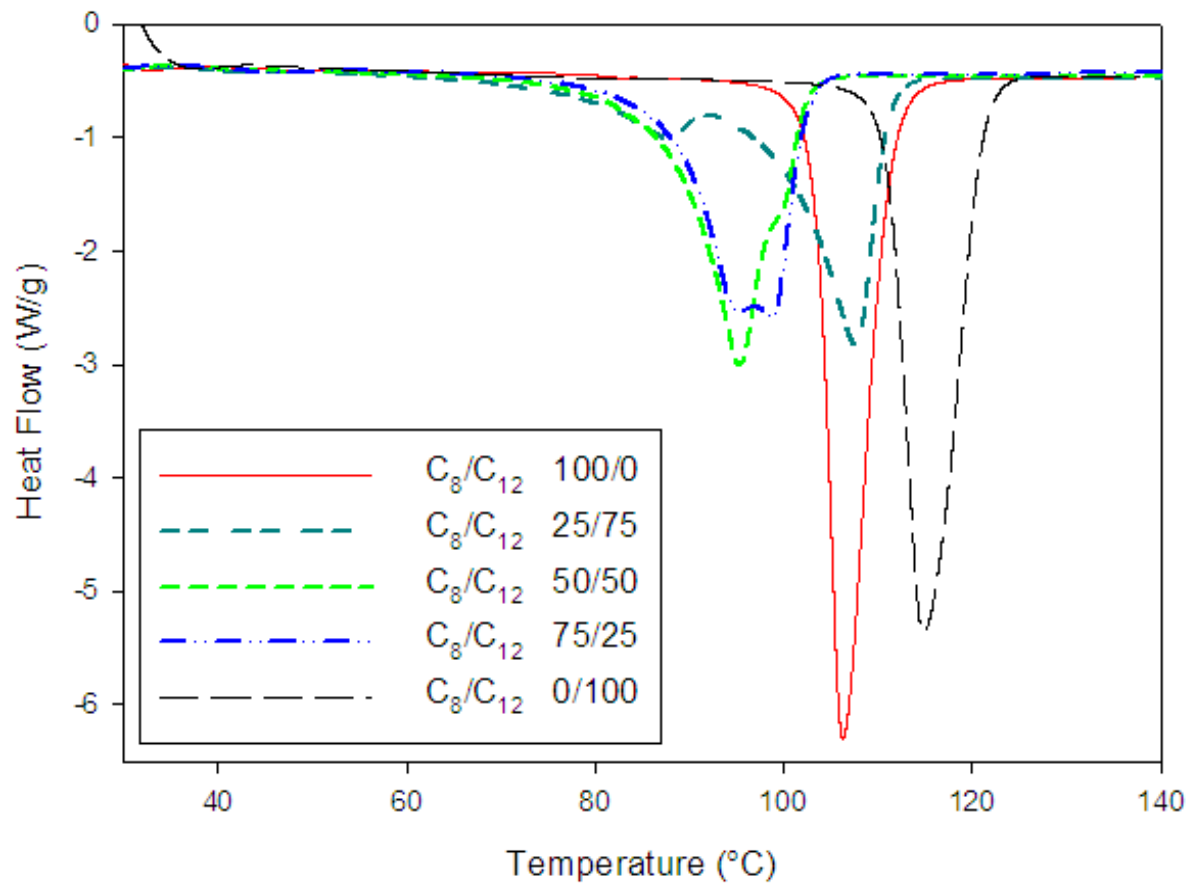

Figure 3.10. DSC thermographs of various concentrations of $\mathrm{C}_{8} / \mathrm{C}_{12}$ blends prepared by thermal annealing.

\subsection{Other Precipitation Methods}

A few other methods were explored to obtain monodisperse blends: (i) a "hotevaporation" method (ii) adding a non-solvent (acetone) to force the precipitation of biscarbamate from the more soluble solution (chloroform), and (iii) by quenching the hot biscarbamate blend solution in an ice bath and then evaporating the chloroform from the blend.

The method of "hot-evaporation" involves heating the solution of the biscarbamates in a sealed vial at around $70-80{ }^{\circ} \mathrm{C}$ and then removing the sample from the hot plate. Without cooling it down to room temperature, the bottle was left open in the fume hood. 
As the chloroform evaporated and left the solution more concentrated, the biscarbamate precipitated out. The blend was placed in a vacuum oven ensuring that all traces of solvent were removed. Unlike the previously used method for the preparation of the blend, the hotevaporation method cannot be quantified in regards to the precipitation rate due to the lack of control of the solvent evaporation and the biscarbamate precipitation. In the case of the addition of the non-solvent, a sample of $\mathrm{C}_{8} / \mathrm{C}_{12}$ biscarbamate solution in chloroform was prepared and set to room temperature. Acetone $(100 \mu \mathrm{L})$ was added to the $3 \mathrm{~mL}$ solution with a syringe at a rate of $10 \mu \mathrm{L}$ per minute. When the acetone-chloroform ratio reached $1: 30$, the sample started to precipitate. Finally, a sample of the blend was prepared by quenching the solution into an ice bath. The blends were then dried for a few days in a fume hood and then 1 day in vacuum. The melting points are listed in Table 3.6.

Table 3.6. Melting points and the full-width-half-max of $\mathrm{C}_{8} / \mathrm{C}_{12} 50 / 50$ blend of biscarbamates prepared by solvent mixing and its improvements. The results indicate only single peaks.

\begin{tabular}{lcccc}
\cline { 2 - 5 } & \multicolumn{3}{c}{$\mathrm{C}_{8}$} & \multicolumn{2}{c}{$\mathrm{C}_{12}$} \\
\hline Method & $\mathrm{T}_{\mathrm{m}},{ }^{\circ} \mathrm{C}$ & FWHM & $\mathrm{T}_{\mathrm{m}},{ }^{\circ} \mathrm{C}$ & $\mathrm{FWHM}$ \\
\hline Solvent Blend & 103.4 & 4.9 & 103.4 & 4.9 \\
Hot-Evaporation & 106.1 & 3.4 & 106.1 & 3.4 \\
Bi-solvent Mixing & 106.3 & 7.2 & 106.3 & 7.2 \\
Quenched Blend & 104.5 & 3.5 & 104.5 & 3.5 \\
\hline
\end{tabular}



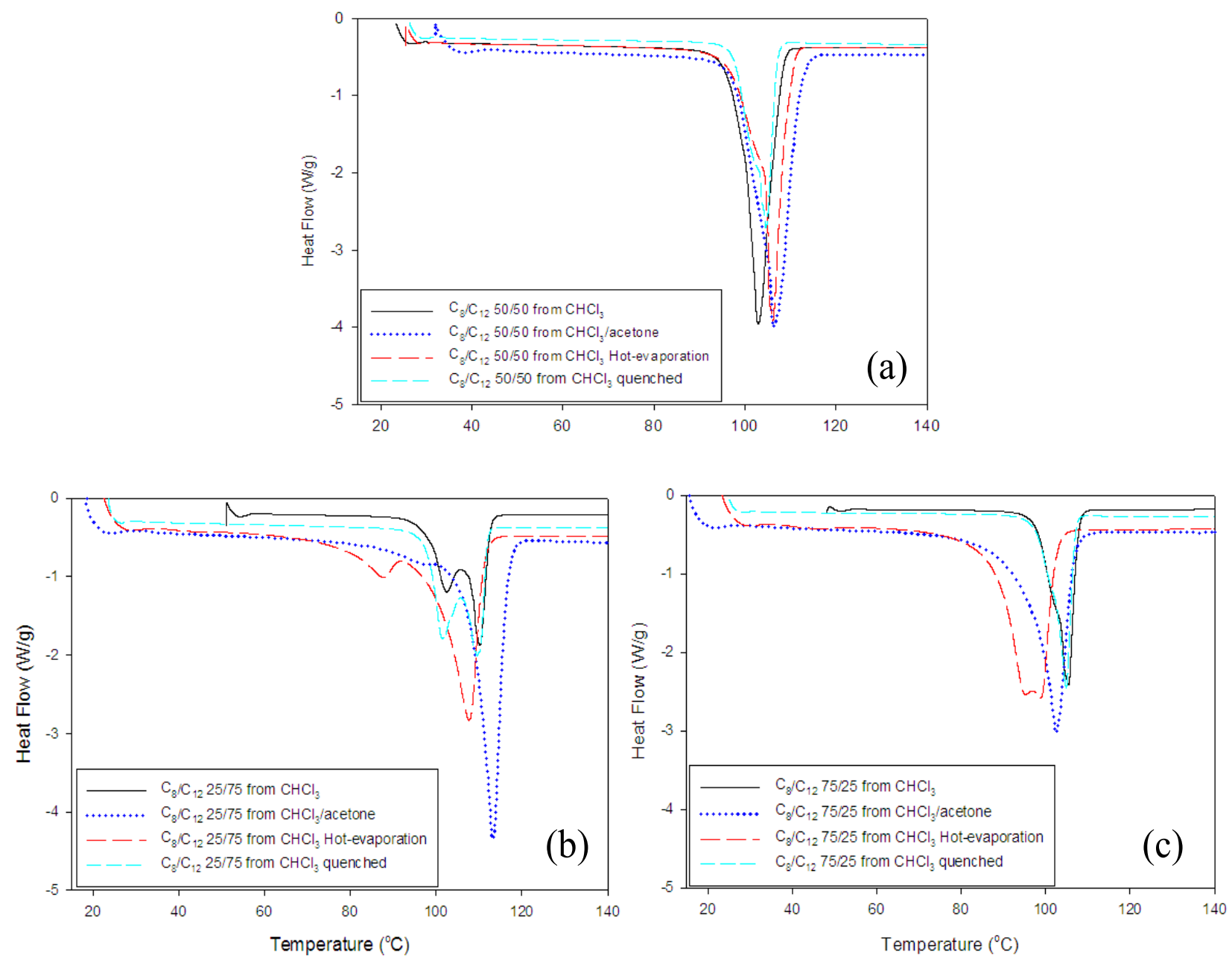

Figure 3.11. DSC endotherms of $\mathrm{C}_{8} / \mathrm{C}_{12}$ biscarbamate (a) $50 / 50$, (b) $25 / 75$ and (c) $75 / 25$ blends prepared by various methods.

The DSC thermographs of the $\mathrm{C}_{8} / \mathrm{C}_{12}$ biscarbamate blends prepared as above are shown in Figure 3.11. The endothermic peaks of the 50/50 blend (Figure 3.11a) prepared from the four methods are singular and narrow except that the bi-solvent and quenched blends show a shoulder. In addition, all four peaks are fairly close to each other in width and in position, with the $\mathrm{T}_{\mathrm{m}}$ ranging from 103.4 to $106.3^{\circ} \mathrm{C}$. This indicates that there is no 
significant change in the self-sorting behaviour. However, comparing with the $\mathrm{T}_{\mathrm{m}}$ 's of the melt-mixed blends (Table 3.2), the extent of depression of $T_{m}$ due to dilution effect is less. With melt-mixing, the $\mathrm{T}_{\mathrm{m}}$ was $96.9^{\circ} \mathrm{C}$, whereas it is 103.4 to $106.3^{\circ} \mathrm{C}$ with the solvent based blends. Thus, the dilution effect also depends on the blend preparation method. Irrespective of the method used for preparing the blend, the $25 / 75$ and $75 / 25$ blends (Figures $3.11 \mathrm{~b}$ and c, respectively) show two endotherms or a broad one with a shoulder.

Although the $50 / 50$ blend of $\mathrm{C}_{8} / \mathrm{C}_{12}$ showed a single endotherm, the same was not seen when the lengths of the alkyl chains are significantly different. The $\mathrm{C}_{4} / \mathrm{C}_{18}: 50 / 50$ blends (Table 3.7) showed two distinct endotherms similar to the temperatures shown in Table 3.2.

Table 3.7. Melting points and the full-width-half-max of $\mathrm{C} 4 / \mathrm{C} 1850 / 50$ blends of biscarbamates prepared by solvent mixing and its improvements. The results indicate only single peaks.

\begin{tabular}{lcccc}
\cline { 2 - 5 } & \multicolumn{3}{c}{$\mathrm{C}_{4}$} & \multicolumn{2}{c}{$\mathrm{C}_{18}$} \\
\hline Method & $\mathrm{T}_{\mathrm{m}},{ }^{\circ} \mathrm{C}$ & FWHM & $\mathrm{T}_{\mathrm{m}},{ }^{\circ} \mathrm{C}$ & $\mathrm{FWHM}$ \\
\hline Solvent Blend & 92.7 & 4.9 & 113.9 & 4.9 \\
Hot-Evaporation & 95.4 & 3.4 & 110.7 & 3.4 \\
Bi-solvent Mixing & 95.2 & 7.2 & 112.8 & 7.2 \\
Quenched Blend & 92.7 & 4.9 & 113.9 & 4.9 \\
\hline
\end{tabular}




\subsection{Odd and Even Biscarbamate Blends}

In a previous publication, ${ }^{13}$ we discussed the effect of carbon atom parity on the thermal and morphological properties of biscarbamates. As illustrated in Scheme 3.1, the terminal methyl groups of hexane are anti to each other, and those of heptane are syn. ${ }^{27}$ With the biscarbamate having even number of carbon atoms in the side chain, the terminal methyl groups are pointing in the same direction (syn) with respect to the ester oxygen, and in the case of those with odd number of carbon atoms the terminal methyl groups are trans to the ester oxygen. This would lead to differences in the packing of terminal methyl groups in the crystal structures of these molecules, giving rise to odd-even effect in the melting temperatures of the biscarbamates.

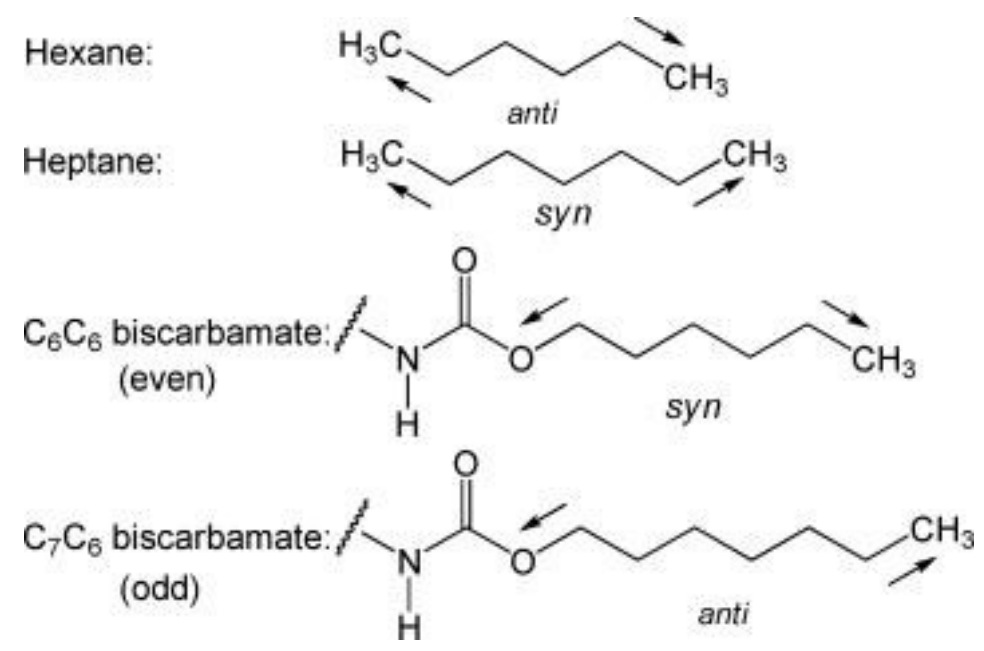

Scheme 3.1. The directions of the terminal methyl groups for even and odd molecules. The terminal methyl groups for hexane are pointing in opposite directions (anti), and those of heptane are in the same direction (syn). Taken from ref 13. 
We explored if mixing an odd and an even biscarbamates with similar $\mathrm{T}_{\mathrm{m}}$ 's and growth rates would lead to molecular association or self-sorting. The melting temperatures of $\mathrm{C}_{3}$ and $\mathrm{C}_{6}$ are 102.2 and $99.5^{\circ} \mathrm{C}$, respectively and their rate of crystallization is abound $40 \mu \mathrm{m} / \mathrm{sec}^{12,13}$ The thermograph of the $50 / 50$ blend (Figure $3.12 \mathrm{a}$ ) shows the endotherms of the individual components near $100^{\circ} \mathrm{C}$. Samples of 50/50 mixtures were prepared using both the melt- and solvent-based blending. The endothermic peaks corresponding to both blends occur at $88^{\circ} \mathrm{C}$ (both lower than the individual components). However, the peak for the melt-based blend is significantly larger in width compared to its solvent-based counterpart. The mutual dilution effect and the lack of a eutectic shows that plasticization rather than co-crystallization occurred. However, a single endotherm is seen when the $T_{m}$ 's and growth rates are similar, although it is an odd-even mixture. While this is observed when the relative concentrations are equal, two separate endotherms are seen in the case of 25/75 (Figure 3.12b) and 75/25 (Figure 3.12c) blends, irrespective of whether the blend was prepared by melt-mixing or via the solution route. Despite both biscarbamates having similar crystallization rates, the relative concentrations of the components need to be balanced to obtain a single endotherm. An abundance of one biscarbamate over the other affects the miscibility of the mixture. It is also seen from Figures $3.12 \mathrm{~b}$ and $\mathrm{c}$ that the depression of the $\mathrm{T}_{\mathrm{m}}$ 's of the components is larger with the melt-mixed blends. 

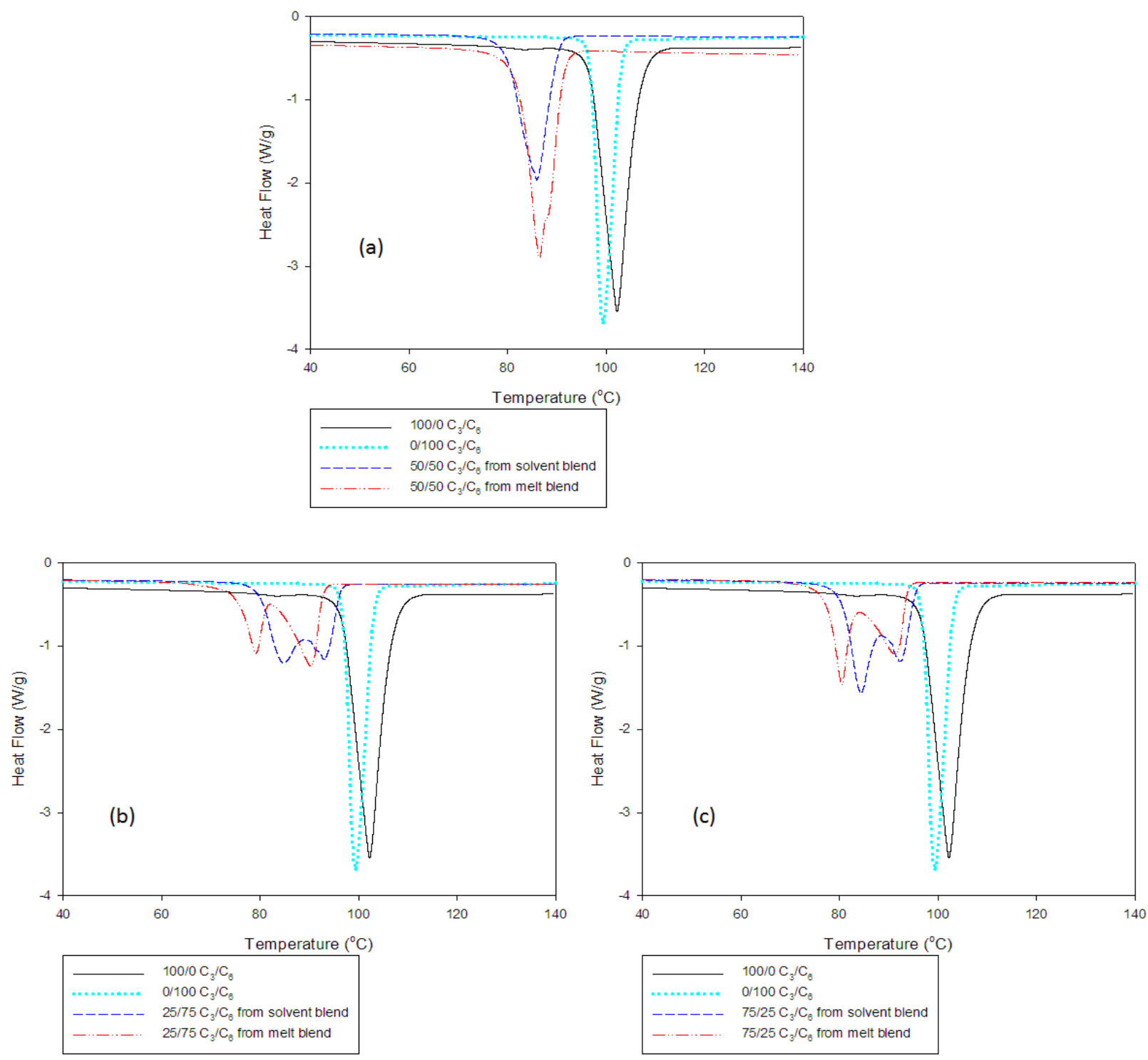

Figure 3.12. Melting points of $\mathrm{C}_{3} / \mathrm{C}_{6}$ biscarbamate blends at (a) $50 / 50$, (b) $25 / 75$ and (c) $75 / 25$ mixture prepared by solvent mixing and melt blend. There is a shoulder on the melt blend of the 50/50 (a) blend thermograph that is not present for the solvent mixing sample. This shoulder is an indication of the sample not fully mixing. The 25/75 (b) and 75/25 (c) blends show multiple peaks for both the solvent- and melt-based blends. This suggests that the abundance of one biscarbamate over the other will affect the miscibility of the mixture. 


\subsection{A Third Component as a Compatibilizer}

It is known that in the case of a blend of two homopolymers A and B, miscibility is an exception rather than a rule due mainly due to entropic factors. It is common practise to use AB block copolymers to compatibilize blends of homopolymers A and B. The results presented above show that mixtures of biscarbamates with about the same side chain lengths yield a single melting endotherm. We examined if a mixture of $\mathrm{C}_{12}$ and $\mathrm{C}_{18}$, with $\mathrm{C}_{16}$ as the compatibilizer would lead to a single melting temperature. Figure 3.13 shows the DSC curves for the melt- and solution mixed blends with $\mathrm{C}_{12} / \mathrm{C}_{16} / \mathrm{C}_{18}: 40 / 20 / 40$ (wt \%). A single endotherm is seen, although the $T_{m}$ is less than any of the three components suggesting plasticization than molecular mixing and co-crystallization. ${ }^{28} \mathrm{We}$ also looked at the addition of a long chain alcohol (pentadecanol) to see if the presence of a long alkyl chain would act as a compatibilizer.

The results indicate that the addition of the $\mathrm{C}_{16}$ biscarbamate does indeed produce a miscible blend as seen from a single narrow endothermic peak for both cases. The melting temperatures lie in between the melting temperature of both $\mathrm{C}_{12}$ and $\mathrm{C}_{18}$, where there is only an intermediate difference between the numbers of carbons along the side chain. This suggests that the diluents effect is diminished in comparison to the two-component blend. The difference in melting temperature for these blends and each individual component is also smaller than the two-component blends. It was observed that the difference of melting temperature for the solvent-based blend is lower than for the melt-based one. Similar to the two-component blend, it would appear that the presence of the solvent is required to help bridge the crystallization rate. In the case of the blend made with pentadecanol, there is a clear splitting of the endotherm representing each individual component. It is clear that 
hydrogen bonds from the biscarbamate are required for the third component to act as a compatibilizer. These results shows indication of the presence of a $3^{\text {rd }}$ component can help with the miscibility of blends.

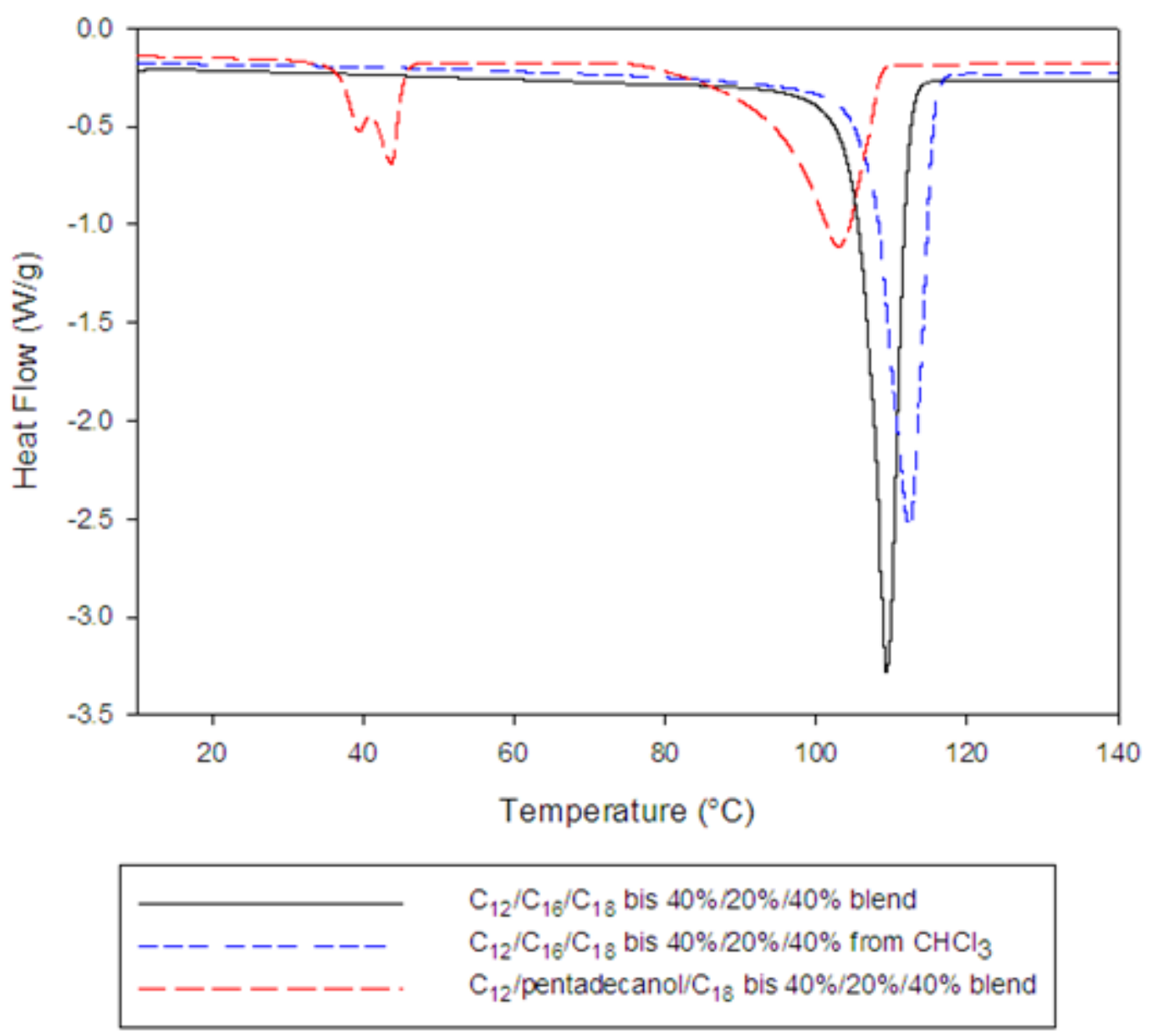

Figure 3.13. DSC thermographs of 3 component blends.

When varying the concentration of the middle component $\left(\mathrm{C}_{16}\right)$, it was observed, using DSC, that the crystallinity and hence the miscibility of the blend for $\mathrm{C}_{12}$ and $\mathrm{C}_{18}$ is improved. The blend of $\mathrm{C}_{12}$ and $\mathrm{C}_{18}$ yields already a single peak, but the change of the 
width and temperature of the peak with the addition of the third component is still noticeable as indicated on Table 3.8. The results indicate the improvement of the crystallinity via the narrowing of the full-width-half-max of the endothermic peak. The FWHM decreases with the addition of $\mathrm{C}_{16}$ until it reaches $20 \mathrm{wt} \%$ of the blend. The FWHM increases dramatically after that, the peak broadens and a shoulder on the peak starts to develop. This suggests that after the addition of $20 \mathrm{wt} \% \mathrm{C}_{16}$, the components begin to selfsort and its own thermal properties are observed on the DSC as the $\Delta \mathrm{T}_{\mathrm{m}}$ increases dramatically.

Table 3.8. Melting points and the full-width-half-max of $\mathrm{C}_{12} / \mathrm{C}_{16} / \mathrm{C}_{18}$ melt blends of biscarbamates with varying $\mathrm{C}_{16}$ concentrations. The results indicate a single peak with varying width.

\begin{tabular}{lll}
\hline$\%$ of $\mathrm{C}_{16}$ & $\mathrm{~T}_{\mathrm{m}},{ }^{\circ} \mathrm{C}$ & $\mathrm{FWHM},{ }^{\circ} \mathrm{C}$ \\
\hline 0 & $108.2^{*}$ & 5.5 \\
5 & $106.9^{*}$ & 4.2 \\
10 & 107.6 & 4.7 \\
15 & 108.7 & 4.7 \\
20 & 109.3 & 3.6 \\
30 & $109.3^{*}$ & 7.7 \\
40 & $110.1^{*}$ & 7.3 \\
\hline * At this point, the peak starts to form a shoulder which would not yield a proper FWHM.
\end{tabular}


Finally, several attempts were made to make $\mathrm{C}_{4}$ and $\mathrm{C}_{18}$ miscible using the three component blend. A series of different middle length biscarbamates were used at a concentration $20 \%$ of the blends and equal parts of $\mathrm{C}_{4}$ and $\mathrm{C}_{18}$ (40\% each) to determine the effectiveness of the third component (Figure 3.14). Since it was previously observed that a concentration of $20 \%$ for the middle component in the $\mathrm{C}_{12} / \mathrm{C}_{16} / \mathrm{C}_{18}$ blend yielded the most miscible blend, we looked at various compositions at that concentration. The results show that these two extreme biscarbamates are too different to produce a miscible blend. The endotherms still produce broad and split peaks showing indication of self-sorting. However, from the thermographs, we do observe the effects of the third component on the individual peak of $\mathrm{C}_{4}$ and $\mathrm{C}_{18}$ component. The $\Delta \mathrm{T}_{\mathrm{m}}$ for each three component blends of $\mathrm{C}_{4} / \mathrm{C}_{\mathrm{X}} / \mathrm{C}_{18}$ has decreased dramatically in comparison to the two-component blend prepared by all the methods previously mentioned. However, the improvements made on the crystallization rate are still not enough to prevent any self-sorting. It is clear that we can improve and control the miscibility with this new procedure with limitations on the difference between the two components in the blend. 

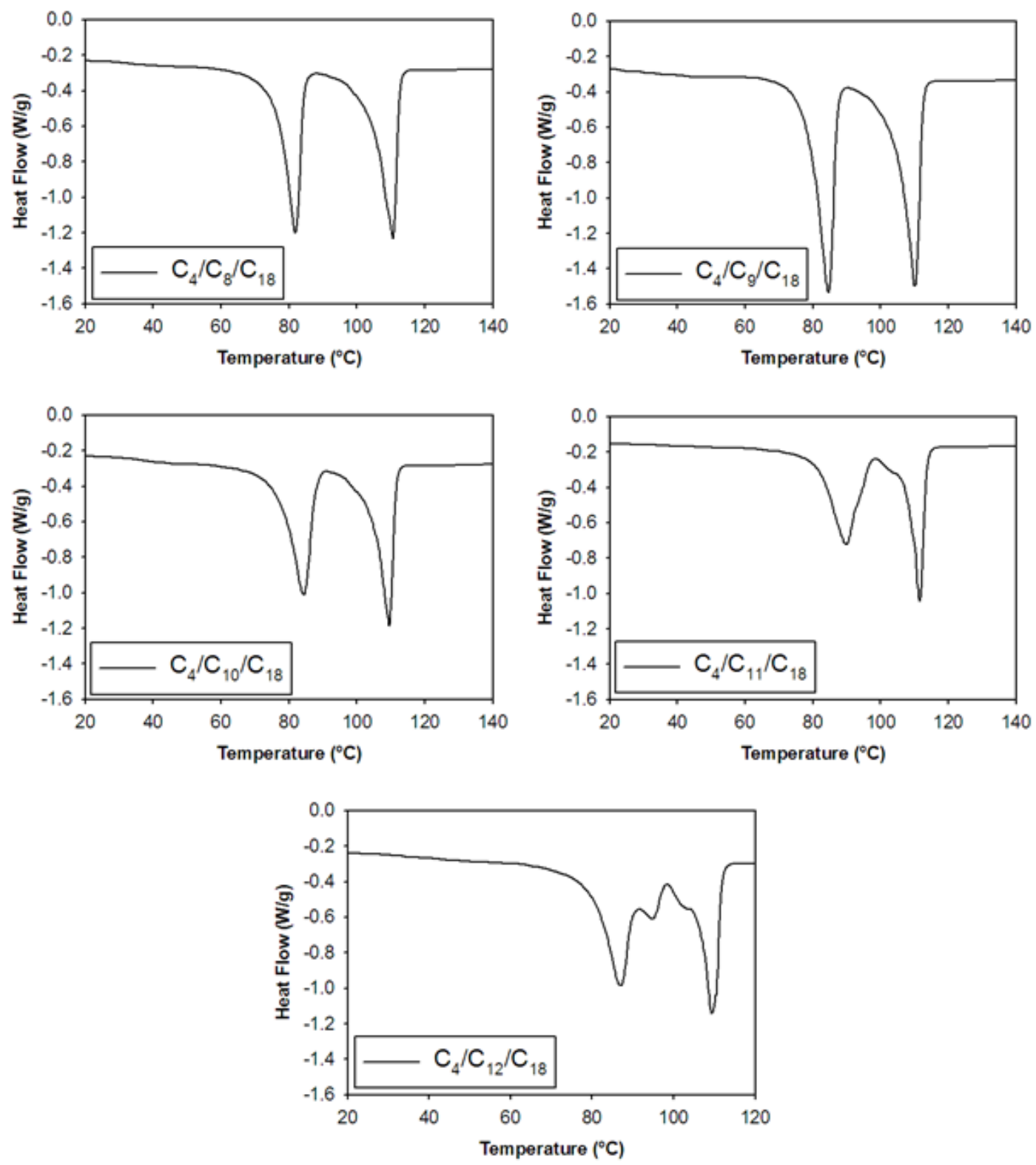

Figure 3.14. DSC thermographs of 3 component blends for $\mathrm{C}_{4} \mathrm{C}_{\mathrm{x}} \mathrm{C}_{18}$.

\subsection{Conclusions}

So far, only even-even biscarbamate blends have been characterized. The reasoning for this is that the behaviour of the odd-odd blends is similar to that of the even-even blends. 
Solvent-based blends of $\mathrm{C}_{3} / \mathrm{C}_{15}, \mathrm{C}_{7} / \mathrm{C}_{11}$ and $\mathrm{C}_{13} / \mathrm{C}_{15}$ were produced to represent biscarbamate blends with varying differences in side chain length. The DSC results showed narrow peaks for all concentration the $\mathrm{C}_{13} / \mathrm{C}_{15}$ blend, wide or peaks with shoulders for the $\mathrm{C}_{7} / \mathrm{C}_{11}$ blend, and multiple peaks for the $\mathrm{C}_{3} / \mathrm{C}_{15}$ blend. As the trend is similar to the eveneven biscarbamate blends, for the purposes of this thesis, we will not go into the details. It was previously observed that melt blending two molecules which are capable of hydrogenbond-mediated self-assembly would often lead to self-sorting. The melting temperatures of the blends are not intermediate between those of the lower and higher melting components, which would otherwise imply miscibility. It was believed that the key to miscibility is the crystallization rate and not the melting temperature of each molecule. In an attempt to control the rate of crystallization, solvent-based blends of two biscarbamates with varying side chain length were made. Improvements on these blends were also attempted: thermal and solvent annealing, slow evaporation and bi-solvent blends, and third component blend.

The results discussed above indicate that solvent mixing can greatly decrease the self-sorting behaviour. It was shown that there is a smaller change in the melting temperatures caused by the diluent effect for the solvent-based blends compared to the melt-based blends. In addition, the crystallization of the blends has changed significantly as seen by the FWHM of the thermographs from the DSC and the XRD patterns. This was an indication of the presence of solvent limiting the self-sorting behaviour by controlling the rate of crystallization. The closer the crystallization rate, the more miscible the blend becomes. It turns out that biscarbamates with similar crystallization rates usually have similar side chains lengths and thus similar melting temperatures. However, we did indeed 
show that is was possible to prepare miscible blends of two molecules with different melting temperatures by using a compatibilizer. As mentioned in the beginning of this chapter, supressing the self-sorting behaviour and preparing miscible blends is important for tuning materials properties. To this end, the approaches discussed in this chapter might be used for specific pairs of the components in the blends. 


\subsection{References}

(1) Khan, M. K.; Sundararajan, P. R. Molecular Selectivity and Immiscibility during the Crystallization of Mixtures of a Set of Homologous Self-Assembling Molecules. $J$. Phys. Chem. B 2008, 112 (14), 4223-4232.

(2) Lehn, J.-M. Toward Self-Organization and Complex Matter. Science (80-. ). 2002, 295 (5564), 2400-2403.

(3) Lehn, J.-M. Programmed Chemical Systems: Multiple Subprograms and Multiple Processing/Expression of Molecular Information. Chem. Eur. J. 2000, 6 (12), 20972102.

(4) Saur, I.; Scopelliti, R.; Severin, K. Utilization of Self-Sorting Processes To Generate Dynamic Combinatorial Libraries with New Network Topologies. Chem. Eur. J. 2006, 12 (4), 1058-1066.

(5) Wu, A.; Isaacs, L. Self-Sorting: The Exception or the Rule? J. Am. Chem. Soc. 2003, 125 (16), 4831-4835.

(6) McKeown, N. B. Phthalocyanine Materials: Synthesis, Structure, and Function; Cambridge University Press, 1998.

(7) Swalen, J. D.; Allara, D. L.; Andrade, J. D.; Chandross, E. A.; Garoff, S.; Israelachvili, J.; McCarthy, T. J.; Murray, R.; Pease, R. F. Molecular Monolayers and Films. A Panel Report for the Materials Sciences Division of the Department of Energy. Langmuir 1987, 3 (6), 932-950.

(8) Weiss, J.; Takhistov, P.; McClements, D. J. Functional Materials in Food 
Nanotechnology. J. Food Sci. 2006, 71 (9), R107-R116.

(9) Goodbrand, B.; Boils, D.; Sundararajan, P. R.; Wong, R.; Malhotra, S. U. S. Patent 6,187,082, 2001.

(10) Moniruzzaman, M.; Goodbrand, B.; Sundararajan, P. R. Morphology and Thermal Behavior of Self-Assembling Carbamates. J. Phys. Chem. B 2003, 107 (33), 84168423.

(11) Goodbrand, B.; Boils, D. C.; Sundararajan, P. R.; Wong, R. W. US Patent 6,414,051, 2002.

(12) Khanna, S.; Moniruzzaman, M.; Sundararajan, P. R. Influence of Single versus Double Hydrogen-Bonding Motif on the Crystallization and Morphology of SelfAssembling Carbamates with Alkyl Side Chains: Model System for Polyurethanes. J. Phys. Chem. B 2006, 110 (31), 15251-15260.

(13) Khan, M. K.; Sundararajan, P. R. Effects of Carbon Atom Parity and Alkyl Side Chain Length on the Crystallization and Morphology of Biscarbamates, A Set of Model Compounds for Polyurethanes. J. Phys. Chem. B 2011, 115 (27), 8696-8706.

(14) Yamaguchi, M.; Miyata, H. Influence of Stereoregularity of Polypropylene on Miscibility with Ethylene-1-Hexene Copolymer. Macromolecules 1999, 32 (18), $5911-5916$.

(15) Jutier, J.; Lemieux, E.; Prud'Homme, R. E. Miscibility of Polyester/nitrocellulose Blends: A DSC and FTIR Study. J. Polym. Sci. Part B Polym. Phys. 1988, 26 (6), 
$1313-1329$.

(16) Vrentas, J. S.; Duda, J. L. Diffusion of Small Molecules in Amorphous Polymers. Macromolecules 1976, 9 (5), 785-790.

(17) Burke Jr, O. W.; Davis, P.; Kizer, J. A. A. Polymerization Process. Google Patents June 27, 1972.

(18) Gránásy, L.; Pusztai, T.; Tegze, G.; Warren, J. A.; Douglas, J. F. Growth and Form of Spherulites. Phys. Rev. E 2005, 72 (1), 11605.

(19) Dickey, K. C.; Anthony, J. E.; Loo, Y. Improving Organic Thin-Film Transistor Performance through Solvent-Vapor Annealing of Solution-Processable Triethylsilylethynyl Anthradithiophene. Adv. Mater. 2006, 18 (13), 1721-1726.

(20) Miller, S.; Fanchini, G.; Lin, Y.-Y.; Li, C.; Chen, C.-W.; Su, W.-F.; Chhowalla, M. Investigation of Nanoscale Morphological Changes in Organic Photovoltaics during Solvent Vapor Annealing. J. Mater. Chem. 2008, 18 (3), 306-312.

(21) Conboy, J. C.; Olson, E. J. C.; Adams, D. M.; Kerimo, J.; Zaban, A.; Gregg, B. A.; Barbara, P. F. Impact of Solvent Vapor Annealing on the Morphology and Photophysics of Molecular Semiconductor Thin Films. J. Phys. Chem. B 1998, 102 (23), 4516-4525.

(22) Li, G.; Yao, Y.; Yang, H.; Shrotriya, V.; Yang, G.; Yang, Y. "Solvent Annealing" Effect in Polymer Solar Cells Based on Poly (3-Hexylthiophene) and Methanofullerenes. Adv. Funct. Mater. 2007, 17 (10), 1636-1644.

(23) Malik, S.; Roberts, C.; Murray, R.; Pate, M. Tuning Self-Assembled InAs Quantum 
Dots by Rapid Thermal Annealing. Appl. Phys. Lett. 1997, 71 (14), 1987-1989.

(24) Sheng, Z.-H.; Shao, L.; Chen, J.-J.; Bao, W.-J.; Wang, F.-B.; Xia, X.-H. CatalystFree Synthesis of Nitrogen-Doped Graphene via Thermal Annealing Graphite Oxide with Melamine and Its Excellent Electrocatalysis. ACS Nano 2011, 5 (6), 43504358.

(25) Ma, W.; Yang, C.; Gong, X.; Lee, K.; Heeger, A. J. Thermally Stable, Efficient Polymer Solar Cells with Nanoscale Control of the Interpenetrating Network Morphology. Adv. Funct. Mater. 2005, 15 (10), 1617-1622.

(26) Liu, G.; Fonash, S. J. Selective Area Crystallization of Amorphous Silicon Films by Low-Temperature Rapid Thermal Annealing. Appl. Phys. Lett. 1989, 55 (7), 660662.

(27) Boese, R.; Weiss, H.-C.; Bläser, D. The Melting Point Alternation in the ShortChain N-Alkanes: Single-Crystal X-Ray Analyses of Propane at $30 \mathrm{~K}$ and of NButane to N-Nonane at 90 K. Angew. Chemie Int. Ed. 1999, 38 (7), 988-992.

(28) Moniruzzaman, M.; Sundararajan, P. R. Role of Hydrogen Bonds in Controlling the Morphology of Self-Assembling Carbamate Systems. J. Phys. Chem. B 2005, 109 (3), 1192-1197. 


\section{CHAPTER 4}

Influence of the Length of the Alkyl Side Chains on the Gelation of Non-Chiral Biscarbamates 


\section{CHAPTER IV: INFLUENCE OF THE LENGTH OF THE ALKYL SIDE CHAINS ON THE GELATION OF NON-CHIRAL BISCARBAMATES}

\subsection{Introduction}

The "odd-even" effect of the carbon number parity of methylene groups in aliphatic chains is a well-known and extensively studied phenomenon. ${ }^{1-5}$ This effect has been observed in the solid state, ${ }^{6,7}$ solution phases, ${ }^{8-10}$ and solid/liquid interfaces ${ }^{11,12}$ and has been mainly attributed to packing differences in the crystal structure ${ }^{13}$ between chains having an odd or even number of carbon atoms. Past studies have shown that compounds such as liquid crystals, ${ }^{14}$ polyurethanes, ${ }^{15}$ n-alkanes, ${ }^{16}$ carboxylic acids, ${ }^{17}$ alcohols, ${ }^{5}$ bifunctional hydroxycarboxylic acids ${ }^{5}$ and many others show odd-even effects as a function of the carbon atom parity. In the overwhelming majority of the literature, this effect is discussed with respect to the carbon atom parity in the spacer groups. ${ }^{18}$ However, there are some reports where this effect is discussed as a function of the carbon atom parity in the alkyl side chains. ${ }^{19,20}$ In most of the cases, the odd-even effect is manifested in the phase transition temperatures ${ }^{21}$ and optical properties ${ }^{22}$ of such compounds.

We previously produced and characterized biscarbamates (scheme 4.1) with an even and odd number of carbon atoms in the side chain (also known as even biscarbamates and odd biscarbamates respectively). ${ }^{23}$ The crystallization behaviour and the morphology of a series of homologous biscarbamate molecules with alkyl side chains of different carbon atom parity were examined. DSC analysis showed that these molecules are highly crystalline in nature. The melting temperatures of the odd biscarbamates versus even biscarbamates show an odd-even alternation. Biscarbamates with an odd number of carbon 
atoms show higher melting temperatures than the even numbered biscarbamates, which is in contrast to the thermal properties of n-alkanes. The heat of fusion shows a relatively less pronounced odd-even effect. Optical microscopy reveals that carbon atom parity in alkyl side chains influences the packing of these molecules leading to a spherulitic morphology. $\mathrm{C}_{4}$ biscarbamate does not show any spherulitic morphology whereas $\mathrm{C}_{3}$ forms well defined spherulites upon crystallization from the melt. It is seen that the spherulite size increases with the increase in alkyl side chain length reaching a maximum and then decreases. Hence the length of alkyl side chain, the carbon atom parity in the alkyl side chains and the sample preparation protocol (i.e., quenching versus slow-cooling) are factored into the morphology of these molecules. Along this series of molecules, we have seen a maximum spherulite size, spherulite growth rate and rate of crystallization for $\mathrm{C}_{7}$ (odd series) and $\mathrm{C}_{8}$ (even series) biscarbamates. This behaviour was justified by the contribution of hydrogen bonding and van der Waals forces as discerned from FTIR spectroscopy. Hence, it was concluded that the thermal properties and the morphology of these molecules can be tailored to the desired functional level by changing the alky side chain length with the right carbon atom parity.

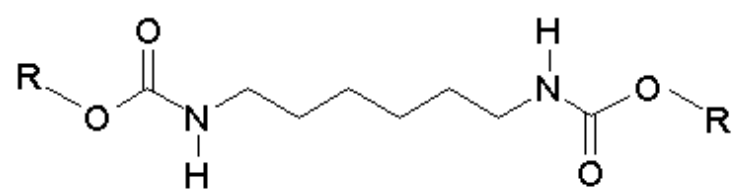

Scheme 4.1. Pictorial representation of a $\mathrm{C}_{6}$ spacer biscarbamate. $\mathrm{R}$ is an alkyl side chain of varying lengths from $C_{3}$ to $C_{18}$. The sample $C_{x}$ refers to the biscarbamate having $X$ number of carbon atoms in the alkyl side chain derived from the appropriate alcohol. 
It was discovered that the control of the self-assembling of the biscarbamates can play a vital role in developing new classes of low molecular weight organic gelators (LMOGs). A gel is solid-like in that it does not flow and can bear a non-zero shear stress, and is liquid-like in that it lacks a structure with long range order. Gels can be easily transformed into solution by heating passed their gel-to-solution temperature $\left(\mathrm{T}_{\text {gel-sol }}\right)$ and back to gel by cooling the solution below their sol-gel transition temperature $\left(\mathrm{T}_{\text {gel }}\right){ }^{24} \mathrm{This}$ reversible property of the gels can be used to make matrices for applications such as controlled release of drugs, ${ }^{25}$ fragrance, ${ }^{26}$ photonic materials, ${ }^{27}$ catalysts, ${ }^{28}$ and is useful in crystal and fuel engineering. ${ }^{29,30}$ Low concentrations of gelators are enough for gel formation, and network filaments are formed by well-ordered arrays of molecules. Gelator molecules self-assemble by highly specific noncovalent interactions, leading to fibrous structures, which form a 3D network. Therefore, self-complementary and unidirectional intermolecular interactions can be considered a prerequisite for the gelation ability. ${ }^{31,32}$ The intermolecular interactions between the organogelator molecules can be from hydrogen bonding, van der Waals interactions, electrostatic attractions, $\pi-\pi$ stacking, or a combination of these.

Gels of even biscarbamates at concentration $0.08 \mathrm{M}$ in benzonitrile were previously made and characterized. ${ }^{33,34}$ It was found that the gels formed short fibres. The shape of the fibres varied from flat ribbons to bent sheets to hollow cylinders to full cylinders depending on the length of the side chains of the biscarbamate. ${ }^{34}$ In a follow up experiment, a gel was produced using a biscarbamate with nine carbons along the side chain $\left(\mathrm{C}_{9}\right)$. In this example, the gel formed long narrow fibres contrary to those found in the even side chain length gels. It is for this reason that there is an interest in looking at the properties of 
odd biscarbamate gels. In this chapter, we examine the compositions, thermal properties and morphology of odd gels.

\subsection{Experimental}

\subsubsection{Synthesis of the Biscarbamates}

The synthesis of biscarbamates of different alkyl side chain lengths was described by Goodbrand et al. ${ }^{35}$ This involved reacting 1,6-diisocyanatohexane $(0.1 \mathrm{~mol})$ with various alcohols $(0.2 \mathrm{~mol})$ ranging from 1-propanol to 1-octadecanol in presence of the catalyst 1,4-Diazabicyclo [2,2,2] octane (DABCO) in toluene. In this chapter, we designate the biscarbamates as $C_{x}$ where $\mathrm{x}$ denotes the number of carbon atoms in the alkyl side chains.

\subsubsection{Preparation of the Gels and Xerogels}

Similar to our group's previous work on mono carbamates ${ }^{36}$ and biscarbamates with even number of carbon atoms in the side chain, ${ }^{33,34}$ benzonitrile was mainly used for the experiments. Gels of concentration $0.08 \mathrm{M}$ (the minimum gelation concentration for the shortest biscarbamate that could gel) were prepared in capped vials to avoid evaporation of the solvent. Weighed amounts of biscarbamates of different alkyl side chain lengths were dissolved in a requisite amount of benzonitrile at a temperature ranging from 80-100 ${ }^{\circ} \mathrm{C}$, which is $20^{\circ} \mathrm{C}$ above the dissolution temperature, to ensure that all biscarbamate nuclei had been removed. Gels were made either by quenching the clear solution in an ice bath or by slowly cooling them to room temperature by turning off the hot plate. The vials were 
turned upside down as a check for gelation: if the samples were not visually separated and did not flow perceptibly, they were considered as gels. All the gels were opaque. These gels were dried at room temperature for a few days and then kept under vacuum for a least a day to form the xerogels.

\section{$\underline{4.2 .3 \text { Characterization of the Methods }}$}

Thermal analysis was performed using a TA Instruments 2010 differential scanning calorimeter at a heating rate of $5{ }^{\circ} \mathrm{C}$ per minute. The calorimeter was calibrated for temperature and energy with an indium sample as a standard reference material. DSC traces were recorded with about 7-8 $\mathrm{mg}$ of xerogel in nitrogen atmosphere. Sample preparation for the wet gels involved putting $30 \mathrm{mg}$ of the wet sample in an aluminium pan $(50 \mu \mathrm{L})$ without the lid and let the sample sit in the fumehood for exactly 30 minutes. Afterwards, the lid was crimped to the pan while making sure no solvent or condensation could escape. Seeing as the temperature range of the DSC run did not go very high (maximum $100{ }^{\circ} \mathrm{C}$ ), deformation of the pan due to excess vapors would not alter our results. The error in the measurements is less than $1{ }^{\circ} \mathrm{C}$. The software used to analyze the thermographs is TA Instruments Universal Analysis 2000 (version 3.9A).

The microscope images were recorded on a Zeiss Axioplan polarized optical microscope (OM). Northern Eclipse (version 6.0) image processing software was used to capture the image. The microscope is equipped with a Linkam hot stage for variable temperature optical microscopy and controlled by a macro from the Northern Eclipse software. This was used to determine the gelation time of the samples.

SEM images of the xerogels were obtained using a JEOL JSM-6400 scanning 
electron microscope. They are then mounted on carbon tape and sputter coated with 80:20 $\mathrm{Au} / \mathrm{Pd}$ alloy. Cryo-SEM was performed on wet samples for aging analysis. The samples were mounted but not coated, and placed in the vacuum chamber at $-30^{\circ} \mathrm{C}$. SEM images were captured at an accelerating voltage ranging from 5 to $20 \mathrm{kV}$. Vega TC software was used for the microscope manipulation and to capture the images.

\subsection{Gelation Behaviour of Biscarbamates}

As the self-assembly is governed by the number of hydrogen bonds in the molecule and the length of the alkyl side chain, there is a competition between the trend towards gelation and crystal formation. A crystal involves a nucleation process and a threedimensional growth based upon equilibrium thermodynamics. A gel is one-dimensional growth leading to a three-dimensional network of flexible chains cross-linked by chemical or physical bonds due to an aggregation in solvent. Biscarbamates with alkyl side chain shorter than $\mathrm{C}_{6}$ do not form a gel; they either remain in solution or precipitate out at higher concentrations. This phenomenon suggests that the contribution of the van der Waals forces due to the side chain length has a strong bearing on the molecules' ability to gel. Based on studies on ureas containing a single hydrogen bonding motif and bisureas with two such sites, Feringa et al. ${ }^{37}$ concluded that the latter is more susceptible to gelation and that increasing the length of the spacer is also effective. The biscarbamates that do form gels remain stable at room temperature for several months as long as they are properly sealed to prevent solvent from escaping.

The minimum concentration $\left(\mathrm{C}_{\min }\right)$ required in each case for gel formation depends 
on the side chain length as well (Figure 4.1). Gelation with the biscarbamates involves the self-assembly by two hydrogen bonding sites and van der Waals interactions between the alkyl side chains in solution. As the length of the alkyl chain increases, the relative contribution from van der Waals interactions is enhanced whereas the number of hydrogen bonds remains the same. Generally for these biscarbamate gels, as the length of the alkyl side chain increases, the $\mathrm{C}_{\min }$ decreases (Figure 4.1) since the van der Waals interactions increase with the chain length. This is in accord with the observation of Abdallah and Weiss ${ }^{16}$ that the gelator efficiency increases with the chain length of simple alkanes. There are two noticeable plateaus along the graph: one where $C_{\min }$ stabilizes between $C_{7}$ to $C_{10}$ and the other between $\mathrm{C}_{12}$ to $\mathrm{C}_{18}$. These two regions illustrate the stability of the gels when either the H-bonding interactions or the van der Waals forces are the main contributor to the gel fibre assembly. It was seen that the variation of the $\mathrm{C}_{\min }$ 's is similar regardless of whether the gel was obtained by quenching or slow cooling. The concentration at which the shortest biscarbamate $\left(\mathrm{C}_{6}\right)$ is able to gel is $0.08 \mathrm{M}$. Hence, for the purpose of this work and to standardize all the biscarbamate gel samples, the concentration is set at that value unless otherwise specified.

The gelation times $\left(\mathrm{G}_{\mathrm{t}}\right)$ for both odd and even biscarbamates prepared by slowcooling are show in Figure 4.2. The $\mathrm{G}_{\mathrm{t}}$ is recorded when the solution first show signs of nucleation growth and ends when the gel is fully set. For both cases, $\mathrm{G}_{t}$ decreases with the increase in the alkyl side chain length. This behaviour, combined with the results shown in Figure 4.1, indicates the significant role of van der Waals interactions in addition to the hydrogen bonds in the gelation process. Interestingly, when comparing $\mathrm{G}_{t}$ between the odd and even biscarbamate, it was observed that odd biscarbamates have a higher gelation time 
than the even carbon atom parity up to the $\mathrm{C}_{12}$ chain length. Beyond $\mathrm{C}_{12}$ chain length, there is a trend reversal: even biscarbamates show higher $G_{t}$ than the corresponding odd biscarbamates. This trend is similar to the crystal growth rates of the biscarbamates (Figures $8 b$ and $9 b$ of ref 23 and 38 respectively). It stands to reason that $\mathrm{G}_{\mathrm{t}}$ (and hence the gelation rate) is affected by the relative van der Waals interactions in a similar manner to those for the crystallization rates.

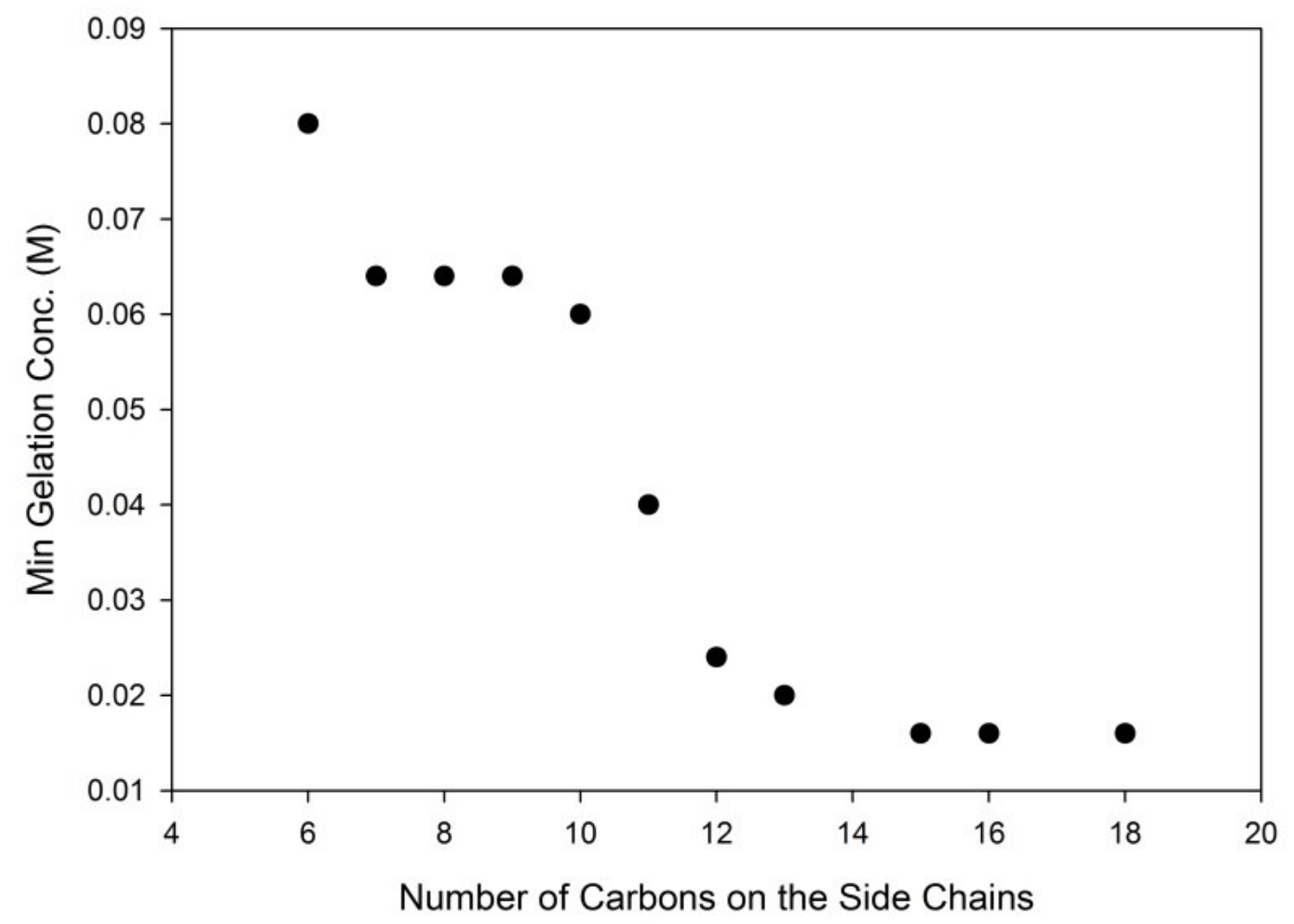

Figure 4.1. The minimum concentration $\left(\mathrm{C}_{\min }\right)$ required for gelation of biscarbamates in benzonitrile. N.B. $\mathrm{C}_{14}$ and $\mathrm{C}_{17}$ biscarbamate were never synthesized due to the limited availability of the reagents. 


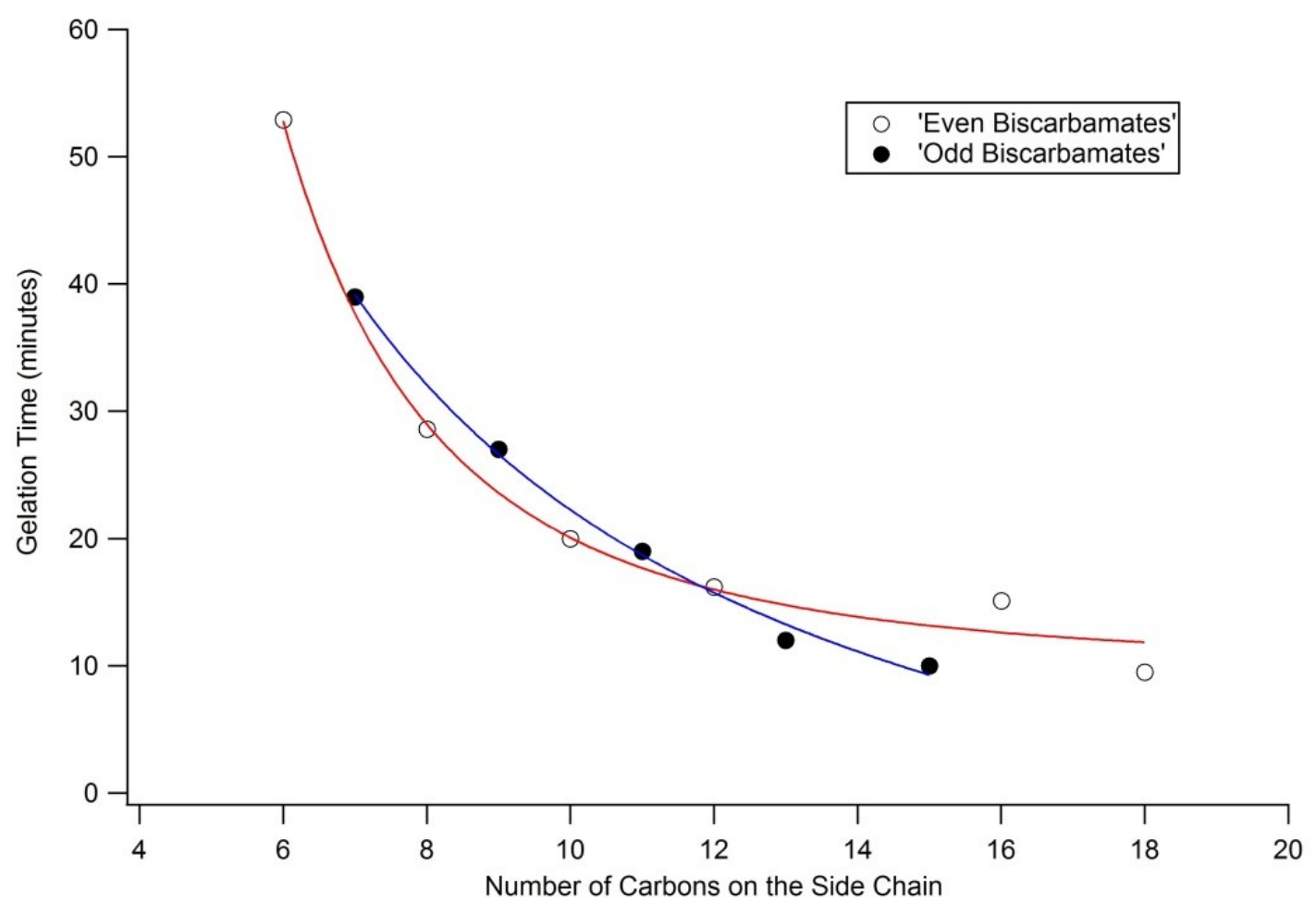

Figure 4.2. Gelation time versus alkyl chain length for both odd and even biscarbamates. Model curves to illustrate the trends were calculated using Igor Pro.

\subsection{Thermal Behaviour of Biscarbamates}

The gel-sol and sol-gel transition were recorded using the DSC. The sample was placed in a sealed aluminum pan and heated at a rate of $5{ }^{\circ} \mathrm{C} / \mathrm{min}$ (pausing at $90^{\circ} \mathrm{C}$ for 30 minutes), cooled at a rate of $5{ }^{\circ} \mathrm{C} / \mathrm{min}$ (pausing at $0{ }^{\circ} \mathrm{C}$ for 30 minutes) and heated again at a rate of $5{ }^{\circ} \mathrm{C} / \mathrm{min}$. For the $\mathrm{C}_{13}$ gel (Figure 4.3), the heat of fusion for the first endotherm is $40.18 \mathrm{~J} / \mathrm{g}$ and the heat of fusion of the second endotherm is $38.84 \mathrm{~J} / \mathrm{g}$ which indicates the reversibility of the gel in relations to temperature. 


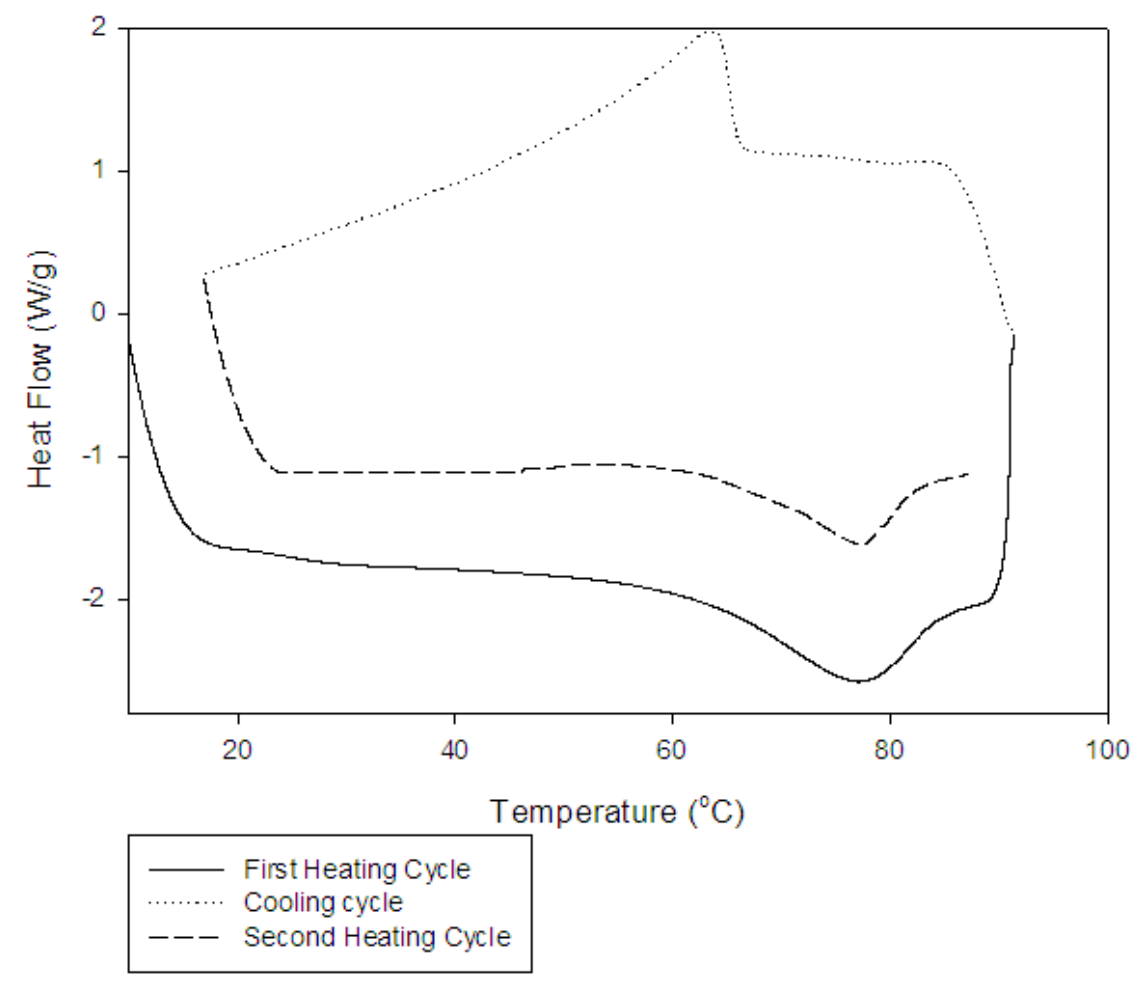

Figure 4.3. DSC thermograph of $\mathrm{C}_{13}(0.08 \mathrm{M})$ wet gel in benzonitrile, heating at a rate of $10{ }^{\circ} \mathrm{C} / \mathrm{min}$ (pausing at $90^{\circ} \mathrm{C}$ for 30 minutes), cooling at a rate of $10{ }^{\circ} \mathrm{C} / \mathrm{min}$ (pausing at 20 ${ }^{\circ} \mathrm{C}$ for 30 minutes) and heating again at a rate of $10{ }^{\circ} \mathrm{C} / \mathrm{min}$. The heat of fusion for the first endotherm is $40.18 \mathrm{~J} / \mathrm{g}$ and the heat of fusion of the second endotherm is $38.84 \mathrm{~J} / \mathrm{g}$.

The temperature of the gel-sol transition depends on the lengths of the side chain and is shown in Figure 4.4. We observe an odd-even effect, with the $\mathrm{T}_{\text {gel }}$ of an odd $\mathrm{C}_{\mathrm{x}}$ being higher than that of succeeding even $\mathrm{C}_{x}$. It appears that even biscarbamates have generally lower gel-sol and sol-gel temperatures than its odd counterpart. Similar to the crystalline melting temperature $\left(\mathrm{T}_{\mathrm{m}}\right)$ of the biscarbamates ${ }^{23}$ the gel-sol temperatures fluctuate depending on having even or odd number of carbon atoms on the side chains. Those with odd $\mathrm{C}_{x}$ side chains showed a higher $\mathrm{T}_{\mathrm{m}}$ and larger spherulites size. We justified this trend 
on the relative conformation of the end groups with respect to the rest of the molecule. As mentioned in the previous chapter, biscarbamates are subject to the effect of carbon atom parity. The terminal methyl groups of hexane are anti to each other, and those of heptane are syn. ${ }^{13}$ The inherent differences in the molecular structure would lead to different packing modes for the odd and even series. We speculate that biscarbamates with odd $\mathrm{C}_{x}$ side chains are more densely packed due to the hydrogen bonding in accordance with dispersion forces. As the melting temperature of the samples is related to $T_{\text {gel-sol, }}$ we observe the same variation in temperature.

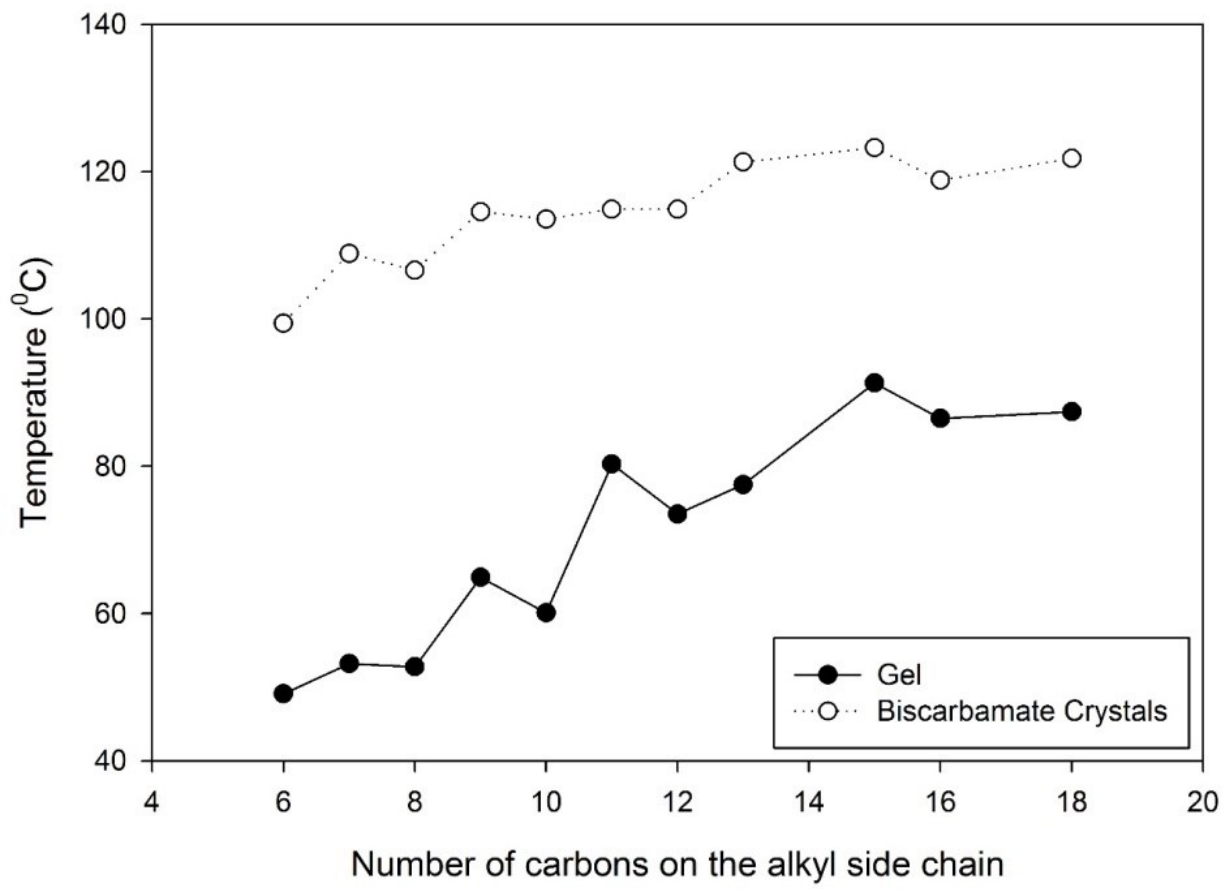

Figure 4.4. The melting temperature of $0.08 \mathrm{M}$ biscarbamate gels and of the respective crystals. N.B. $\mathrm{C}_{14}$ and $\mathrm{C}_{17}$ biscarbamate were never synthesized due to the limited availability of the reagents. 
The formation of gel fibres is due to rapid one-dimensional growth whereas threedimensional growth would result in crystal formation. This is reflected in the morphology of the biscarbamate gels discussed below.

\subsection{Morphology of Biscarbamate Gels}

We analyzed the fibres for the slow-cooled and quenched gels to see if there are any major differences in the lengths of these fibres depending on the method of which the gels are made (Figure 4.5). The slow-cooled gels produced longer fibres compared to the quenched samples. On a closer look, the fibres appear to be hollow tubes or bent sheets. The fibres of the quenched gels are much denser and thinner compared to those of the slowcooled samples. Quenching the solution while still warm forces more nucleation but the fibre growth terminates sooner which yields the denser but thinner fibres. In addition, without the ability to form a thermodynamically favourable structure such as closed tubes, the quenched gel fibres are only able to form stacked thin ribbons or bent fibres. Both gels are stable for the sample period of time of multiple months as long as they are properly sealed in vial. For other solvents, both gelation methods yield the same networking structures (fibres, sheets or rods) but with varying sizes and density. For the purpose of this chapter, we will focus more on the results for the slow-cooled gel samples as we believe that they have the preferred thermodynamic structures. 

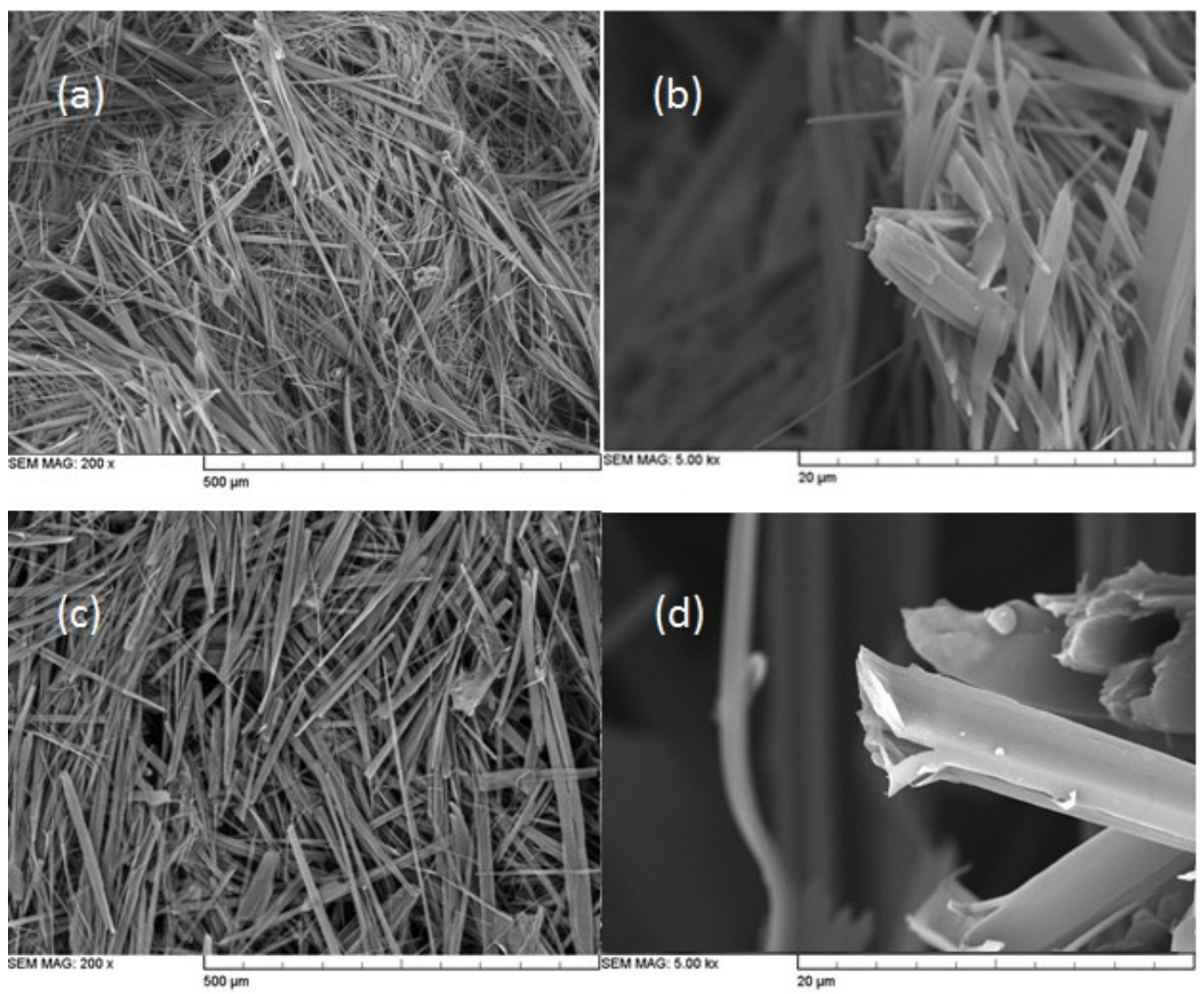

Figure 4.5. SEM images of $\mathrm{C}_{9}$ biscarbamate (a and b) quenched (low and high magnification) and (c and d) slow-cooled (low and high magnification) (0.08 M) prepared from benzonitrile.

SEM images were taken for biscarbamates $\mathrm{C}_{7}$ to $\mathrm{C}_{15}$ slow-cooled xerogels (Figure 4.6). Morphological characterization of the even gels can be found in our previous work. ${ }^{34}$ We chose to examine the xerogel as opposed to any other methods of fibre isolation since it was the least complicated. The precipitation of the biscarbamate molecule in solution would be completely negligible compared to the fibre network already present within the gel. The structure of the fibres would collapse but still retain its general shape. 
The images showed a combination of sheets and ribbons with various lengths. The $\mathrm{C}_{7}$ gel displays the shortest fibres with an average of $250 \mu \mathrm{m}$ length. The length of the fibres increases as the length of the side chain increases until $\mathrm{C}_{13}$ where the length starts to decrease. Table 4.1 lists the average lengths of the slow-cooled odd biscarbamate gels. The structure of the fibres changes dramatically from flat ribbons to bents sheets to tubes to filled ribbons.

Table 4.1. The average lengths of the odd biscarbamate gels in benzonitrile at $0.08 \mathrm{M}$ prepared using the slow-cooled method.

\begin{tabular}{cc}
\hline Biscarbamate & Average Fibre Length $(\mu \mathrm{m})$ \\
\hline $\mathrm{C}_{7}$ & 250 \\
$\mathrm{C}_{9}$ & 462 \\
$\mathrm{C}_{11}$ & 544 \\
$\mathrm{C}_{13}$ & 620 \\
$\mathrm{C}_{15}$ & 513 \\
\hline
\end{tabular}




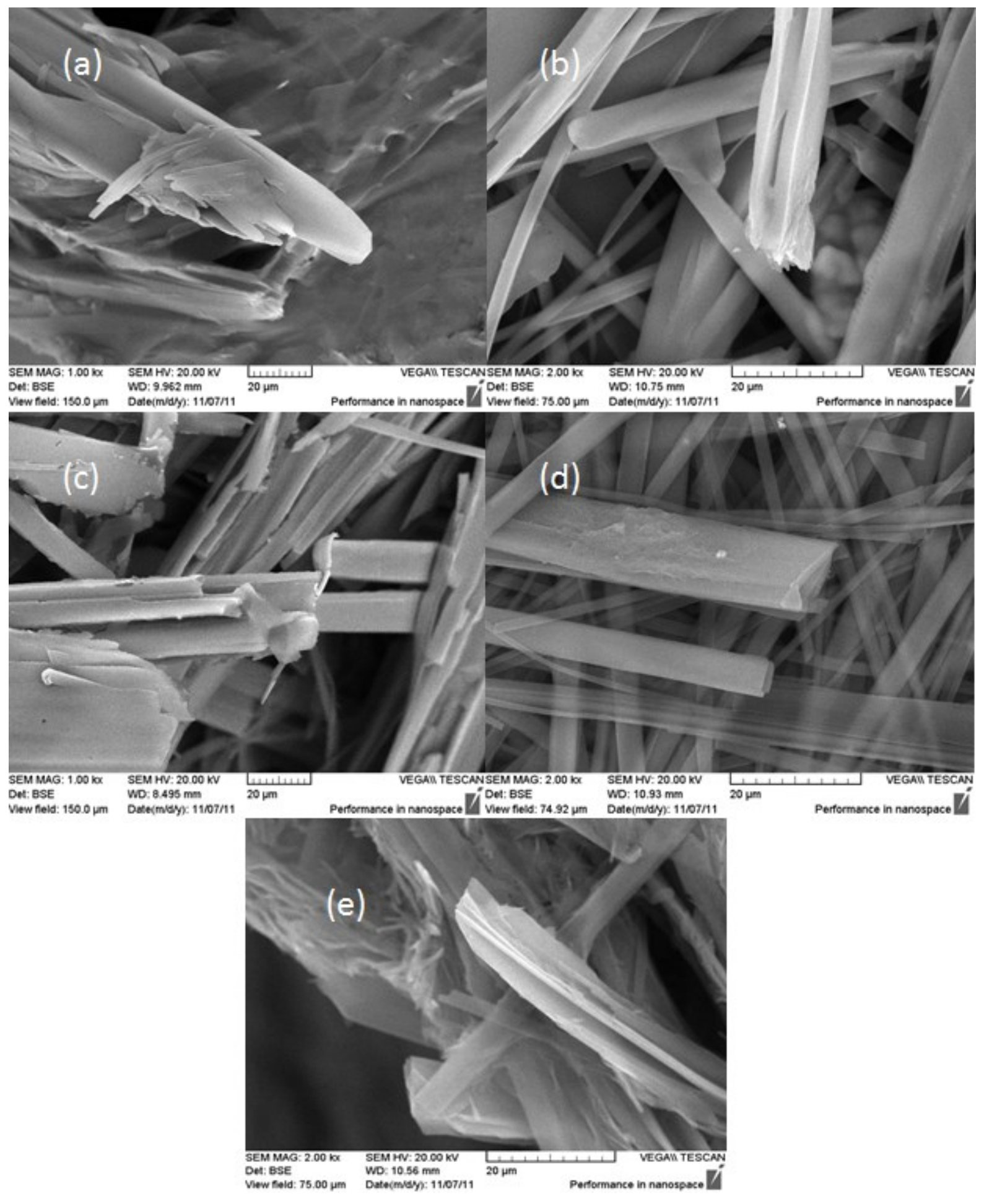

Figure 4.6. SEM images of xerogels of (a) $\mathrm{C}_{7}$, (b) $\mathrm{C}_{9}$, (c) $\mathrm{C}_{11}$, (d) $\mathrm{C}_{13}$ and (e) $\mathrm{C}_{15}(0.08 \mathrm{M})$ prepared from benzonitrile. All samples were slow-cooled, dried overnight and then left in the vacuum oven for a day. 
In the previous section, we briefly discussed the relative contributions of the hydrogen bond and van der Waals interactions. Bending fibres are seen in the gels of odd biscarbamates, although the system is neither chiral nor amphiphilic. They are analogous to the hollow fibres seen for the even biscarbamates. It was hypothesized that due to the predominant van der Waals interaction between the long alkyl chains, in addition to the double hydrogen bonding in biscarbamates, the morphology consists of sheets and tubules, in contrast to long fibres seen in the case of mono carbamates and other organogelators. The formation of tubes have been theorised by Helfrich and Prost,${ }^{39}$ who state that an intrinsic bending force due to chirality would lead a tilted chiral bilayer to form a cylinder. Incomplete wrapping shows the eaves trough morphology. While the tubules seen in the case of chiral molecules were attributed to the twisting caused by chirality, the bending of the sheets in the biscarbamate gels could be justified by the asymmetry of the strength of the following interactions: (i) a dominant force of hydrogen bonding as well as the van der Waals interaction between the long alkyl chains along the gel tape growth direction, (ii) weak interaction between essentially the terminal methyl groups in the direction normal to the gel tape growth direction, and (iii) van der Waals interaction between the sheets of hydrogen bond in the third direction. We propose that when these sheets, when the gel is wet, prefer not to be flat but curl and wrap into bended fibres.

There is an interesting observation where the lengths of the ribbons for the odd gels are significantly longer than those for the even gels. For example, when comparing fibre lengths and width of $\mathrm{C}_{8}$ and $\mathrm{C}_{9}$ gels (Figure 4.7), the fibres for the odd gel are significantly longer (average length of 320 and $462 \mu \mathrm{m}$ for $\mathrm{C}_{8}$ and $\mathrm{C}_{9}$ respectively) while the even gel fibres are thicker (average width of 16 and $8 \mu \mathrm{m}$ for $\mathrm{C}_{8}$ and $\mathrm{C}_{9}$ respectively). The images 
show ribbons where the slow-cooled samples are significantly longer than the quenched samples for both biscarbamates.

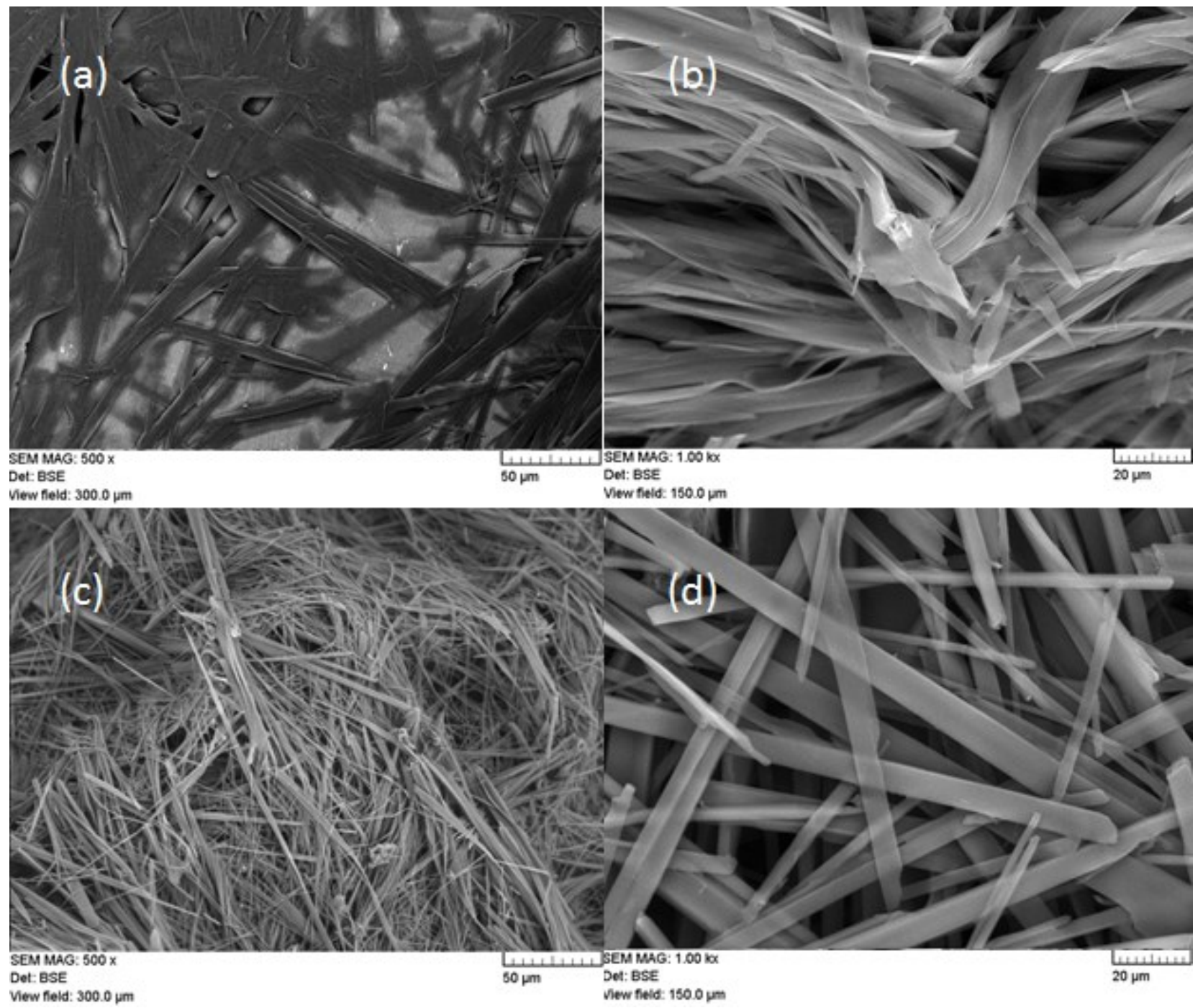

Figure 4.7. SEM images of xerogels of ( $a$ and $b) C_{8}$ and (c and d) $C_{9}(0.08 \mathrm{M})$ prepared from benzonitrile. Both samples were slow-cooled, dried in the fumehood overnight and in the vacuum oven for a day. 
As previously explained, biscarbamates $\mathrm{C}_{8}$ and $\mathrm{C}_{9}$ are very similar in length. However, due to the one extra carbon along the side chain, the packing order is quite different. In the case for even biscarbamates, the terminal methyl groups are pointing in opposite directions (anti), and those of odd biscarbamates are in the same direction (syn) as showed in Scheme 3.1 in Chapter 3. As seen for biscarbamate crystal structure, the result leads to differences in the packing of terminal methyl groups in the fibre formation of these molecules. The even-numbered n-alkanes have symmetrical intermolecular contacts at both ends, whereas the odd numbered ones have a longer distance of contact at one end. ${ }^{13}$

Finally, in a recent study, the Guenet group has developed a calculation to assess the critical gelation concentration of the fibres in gel. ${ }^{40}$ This relates the fibril cross section and the fibril length to $\mathrm{C}_{\min }$ (or $\mathrm{C}_{\mathrm{gel}}$ as they put it) in the following manner:

$$
\mathrm{C}_{\min }=\frac{6 \rho \mathrm{r}^{2}}{\mathrm{~L}_{\mathrm{F}}^{\left(3 / \mathrm{D}_{\mathrm{f}}-1\right)}}
$$

where $r$ is the fibril cross section and $D_{f}$ is the fractal dimension of the long axis of the fibril length $L_{f}$. In the present case $D_{f}=1 . L_{f}$ and $r$ are related so that the smaller $r$ the longer $L_{f}$ and, correspondingly, the smaller $\mathrm{C}_{\min }$. The approximation works for biscarbamate gels in benzonitrile since it produces long fibres. From the data previously observed, there is a general decrease in $\mathrm{C}_{\min }$ as the length of the side chain increases. However, there is no real correlation between the number of carbon on the side chain and the dimensions of the fibres. We observed that if the fibres get too long, the cross section 
decreases in response. But the resulting $\mathrm{C}_{\min }$ still decreases for increasing length. In any case, the $\mathrm{C}_{\min }$ values were obtained from experiment and not from estimation.

\subsection{Gel Aging}

We then looked at the development of the gels following its formation. Both slowcooled and quenched gels of $\mathrm{C}_{7}$ were monitored. The wet gel samples were put under cryoSEM in order to halt further development from either aging or solvent evaporation. The analysis was performed one day, one week and two weeks after the formation of the gel. Figures 4.8 and 4.9 illustrate the change within that time period for the slow-cooled and quenched $\mathrm{C}_{7}$ gels respectively. The two methods did not significantly affect the aging of these gels. One would assume that, given enough time to self-assemble, the fibres of the quenched sample would be able to realign and form defined structures such as the tubes found in the slow-cooled samples. However, no such structures were observed after such time has passed. The final structures were still denser and smaller than those observed for the slow-cooled sample. It would appear that the initial assembly of the molecules into fibres would control the final structure suggesting that the method of preparation is a key step in determining the fine shape of the gel network. The only observable change throughout this period is that the growth of the fibres has somewhat increased and that the fibres are stacking on top of each other. 


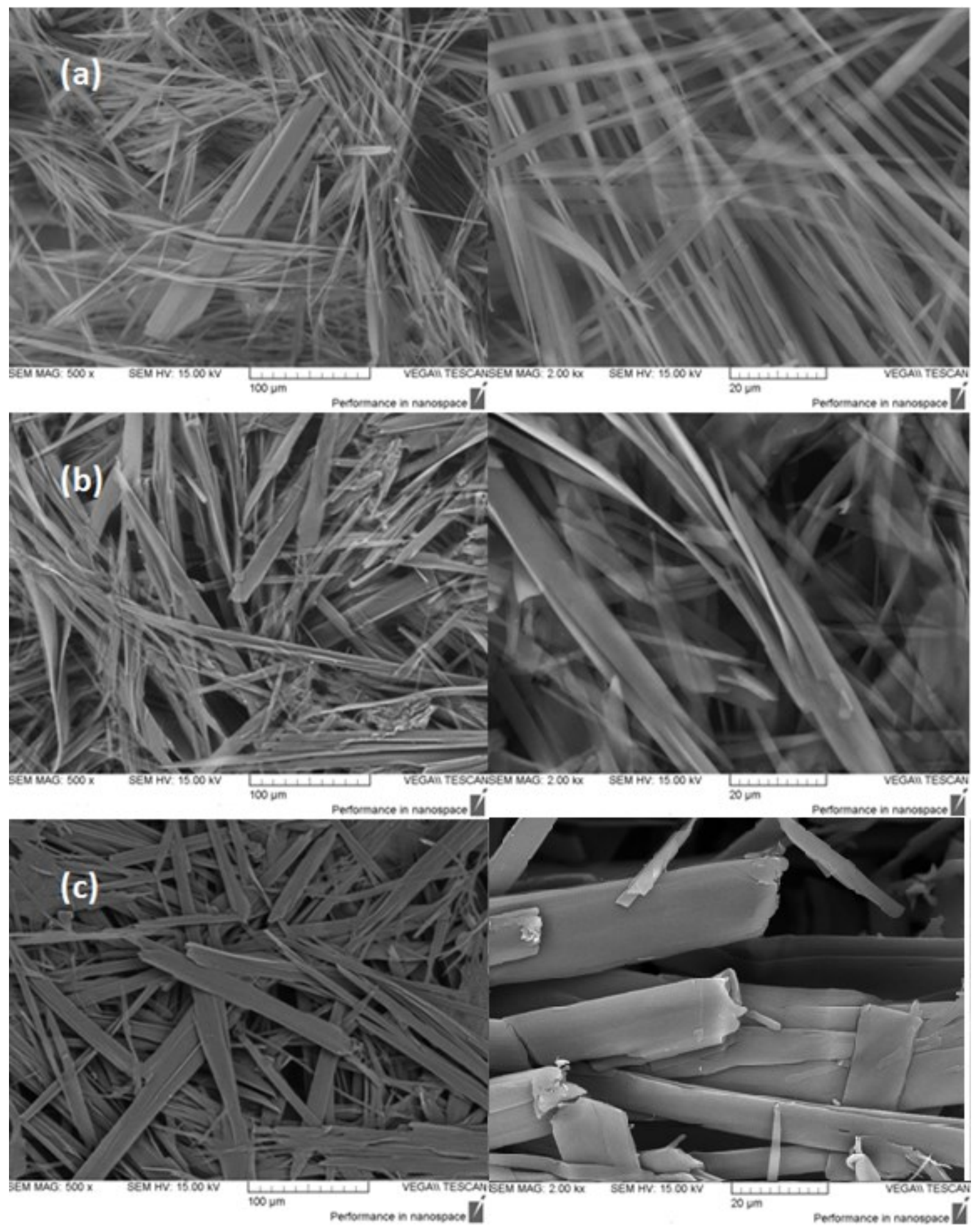

Figure 4.8. Low (left) and high (right) resolution cryo-SEM images of wet $\mathrm{C}_{7}$ gels prepared using the slow-cooling method after (a) 1 day, (b) 1 week, and (c) 2 weeks after formation. 

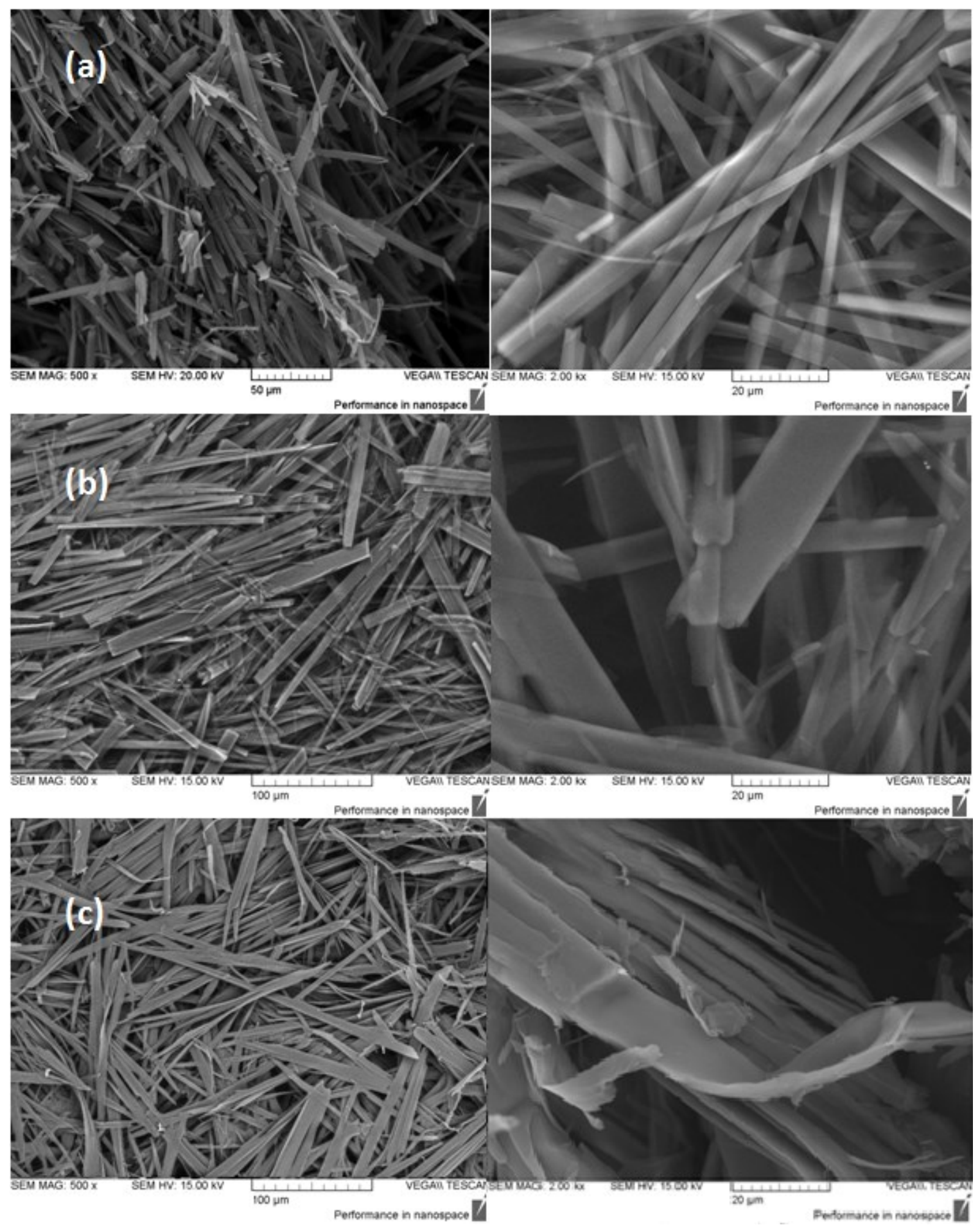

Figure 4.9. Low (left) and high (right) resolution cryo-SEM images of wet $\mathrm{C}_{7}$ gels prepared using the quenching method after (a) 1 day, (b) 1 week, and (c) 2 weeks after formation. 


\subsection{Conclusions}

We discussed the physical properties and the morphology of a series of gels made from homologous biscarbamate molecules with alkyl side chains of different carbon atom parity. The critical gelation concentration increases with greater side chain lengths at constant concentration. DSC analysis showed that all of the biscarbamate gels are thermoreversible. Generally, the $\mathrm{T}_{\text {gel-sol }}$ 's increase as the molecule size increases. However, we do observe an oscillation of temperatures due the difference in stacking order for odd and even biscarbamates. Optical and scanning electron microscopy reveals that carbon atom parity in alkyl side chains influences the packing of these molecules leading to a difference in morphology. It is seen that the fibre size increases with the increase in alkyl side chain length reaching a maximum at $\mathrm{C}_{13}$ and then decreases. In addition, it seems like the length of the ribbons is altered slightly by the preparation method (whether the gels were quenched or slow-cooled). These methods did not affect the aging, the thermoproperties and the stabilities of these gels. We also looked at the growth of the ribbons at various time lengths. It appears that the fibres continue to grow after the formation, but the growth dramatically decreases after a week. Hence, we conclude that the thermal properties and the morphology of these molecules can be tailored to the desired functional level by changing the alkyl side chain length with the right carbon atom parity. 


\subsection{References}

(1) Watanabe, J.; Nakata, Y.; Simizu, K. Frustrated Bilayer Smectic Phase in MainChain Polymers with Two Different Spacers. J. Phys. II 1994, 4 (4), 581-588.

(2) Kobayashi, T.; Seki, T. Odd-Even Effects in Azobenzene-Urea Amphiphile Assemblies. Langmuir 2003, 19 (22), 9297-9304.

(3) Egbe, D. A. M.; Ulbricht, C.; Orgis, T.; Carbonnier, B.; Kietzke, T.; Peip, M.; Metzner, M.; Gericke, M.; Birckner, E.; Pakula, T. Odd-Even Effects and the Influence of Length and Specific Positioning of Alkoxy Side Chains on the Optical Properties of PPE-PPV Polymers. Chem. Mater. 2005, 17 (24), 6022-6032.

(4) Cyganik, P.; Szelagowska-Kunstman, K.; Terfort, A.; Zharnikov, M. Odd- Even Effect in Molecular Packing of Biphenyl-Substituted Alkaneselenolate SelfAssembled Monolayers on Au (111): Scanning Tunneling Microscopy Study. J. Phys. Chem. C 2008, 112 (39), 15466-15473.

(5) Tao, F.; Bernasek, S. L. Understanding Odd-Even Effects in Organic SelfAssembled Monolayers. Chem. Rev. 2007, 107 (5), 1408-1453.

(6) Duer, M. J.; Roper, C. A Solid-State NMR Investigation of the Odd-even Effect in a Series of Liquid-Crystal Dimers. Phys. Chem. Chem. Phys. 2003, 5 (14), 30343041.

(7) Badea, E.; Della Gatta, G.; D’Angelo, D.; Brunetti, B.; Rečková, Z. Odd-even Effect in Melting Properties of 12 Alkane- $\alpha, \omega$-Diamides. J. Chem. Thermodyn. 
2006, 38 (12), 1546-1552.

(8) Stals, P. J. M.; Smulders, M. M. J.; Martín-Rapún, R.; Palmans, A. R. A.; Meijer, E. W. Asymmetrically Substituted Benzene-1, 3, 5-Tricarboxamides: Self-Assembly and Odd-Even Effects in the Solid State and in Dilute Solution. Chem. Eur. J. 2009, 15 (9), 2071-2080.

(9) Pistolis, G.; Andreopoulou, A. K.; Malliaris, A.; Kallitsis, J. K. Direct Observation of Odd-Even Effect in Dilute Polymeric Solutions: A Time-Resolved Fluorescence Anisotropy Study. J. Phys. Chem. B 2005, 109 (23), 11538-11543.

(10) Meister, A.; Drescher, S.; Garamus, V. M.; Karlsson, G.; Graf, G.; Dobner, B.; Blume, A. Temperature-Dependent Self-Assembly and Mixing Behavior of Symmetrical Single-Chain Bolaamphiphiles. Langmuir 2008, 24 (12), 6238-6246.

(11) Tong, W.; Wei, Y.; Armbrust, K. W.; Zimmt, M. B. Dipolar Side Chain Control of Monolayer Morphology: Symmetrically Substituted 1, 5-(Mono-and Diether) Anthracenes at the Solution- HOPG Interface. Langmuir 2009, 25 (5), 2913-2923.

(12) Kikkawa, Y.; Koyama, E.; Tsuzuki, S.; Fujiwara, K.; Kanesato, M. Bipyridine Derivatives at a Solid/Liquid Interface: Effects of the Number and Length of Peripheral Alkyl Chains. Langmuir 2009, 26 (5), 3376-3381.

(13) Boese, R.; Weiss, H.-C.; Bläser, D. The Melting Point Alternation in the ShortChain N-Alkanes: Single-Crystal X-Ray Analyses of Propane at $30 \mathrm{~K}$ and of NButane to N-Nonane at 90 K. Angew. Chemie Int. Ed. 1999, 38 (7), 988-992.

(14) Šepelj, M.; Lesac, A.; Baumeister, U.; Diele, S.; Bruce, D. W.; Hameršak, Z. 
Dimeric Salicylaldimine-Based Mesogens with Flexible Spacers: Parity-Dependent Mesomorphism. Chem. Mater. 2006, 18 (8), 2050-2058.

(15) Versteegen, R. M.; Sijbesma, R. P.; Meijer, E. W. [n]-Polyurethanes: Synthesis and Characterization. Angew. Chemie Int. Ed. 1999, 38 (19), 2917-2919.

(16) Abdallah, D. J.; Weiss, R. G. N-Alkanes Gel N-Alkanes (and Many Other Organic Liquids). Langmuir 2000, 16 (2), 352-355.

(17) Tao, F.; Goswami, J.; Bernasek, S. L. Self-Assembly and Odd-Even Effects of CisUnsaturated Carboxylic Acids on Highly Oriented Pyrolytic Graphite. J. Phys. Chem. B 2006, 110 (9), 4199-4206.

(18) Kim, K.; Plass, K. E.; Matzger, A. J. Structure of and Competitive Adsorption in Alkyl Dicarbamate Two-Dimensional Crystals. J. Am. Chem. Soc. 2005, 127 (13), $4879-4887$.

(19) Goel, M.; Jayakannan, M. Supramolecular Liquid Crystalline $\pi$-Conjugates: The Role of Aromatic $\pi$-Stacking and van Der Waals Forces on the Molecular SelfAssembly of Oligophenylenevinylenes. J. Phys. Chem. B 2010, 114 (39), 1250812519.

(20) Wei, Y.; Kannappan, K.; Flynn, G. W.; Zimmt, M. B. Scanning Tunneling Microscopy of Prochiral Anthracene Derivatives on Graphite: Chain Length Effects on Monolayer Morphology. J. Am. Chem. Soc. 2004, 126 (16), 5318-5322.

(21) Mather, P. T.; Jeon, H. G.; Han, C. D.; Chang, S. Odd-Even Effect of Flexible Spacer Length on Flow-Induced Isotropic-to-Nematic Transition in Segmented 
Thermotropic Polymers. Macromolecules 2002, 35 (4), 1326-1335.

(22) Mizuno, M.; Hirai, A.; Matsuzawa, H.; Endo, K.; Suhara, M.; Kenmotsu, M.; Han, C. D. Study of Odd-Even Effect of Flexible Spacer Length on the Chain Dynamics of Main-Chain Thermotropic Liquid-Crystalline Polymers Using High-Resolution Solid-State 13C Nuclear Magnetic Resonance Spectroscopy. Macromolecules 2002, 35 (7), 2595-2601.

(23) Khan, M. K.; Sundararajan, P. R. Effects of Carbon Atom Parity and Alkyl Side Chain Length on the Crystallization and Morphology of Biscarbamates, A Set of Model Compounds for Polyurethanes. J. Phys. Chem. B 2011, 115 (27), 8696-8706.

(24) Raghavan, S. R. Distinct Character of Surfactant Gels: A Smooth Progression from Micelles to Fibrillar Networks†े. Langmuir 2009, 25 (15), 8382-8385.

(25) Peer, D.; Karp, J. M.; Hong, S.; Farokhzad, O. C.; Margalit, R.; Langer, R. Nanocarriers as an Emerging Platform for Cancer Therapy. Nat. Nanotechnol. 2007, $2(12), 751-760$.

(26) Kunze, W. A. Extended Release Hot and Cold Gel Fragrance Cartridges and Method of Making the Same. US Patent 5575992 A 1996.

(27) Arsenault, A. C.; Míguez, H.; Kitaev, V.; Ozin, G. A.; Manners, I. A Polychromic, Fast Response Metallopolymer Gel Photonic Crystal with Solvent and Redox Tunability: A Step towards Photonic Ink (P-Ink). Adv. Mater. 2003, 15 (6), 503507.

(28) Bailey, G. C.; Holm, V. C. F. Sulfate-Treated Zirconia-Gel Catalyst. US Patent 
3032599 A 1962.

(29) Buerkle, L. E.; Rowan, S. J. Supramolecular Gels Formed from Multi-Component Low Molecular Weight Species. Chem. Soc. Rev. 2012, 41 (18), 6089-6102.

(30) Sangeetha, N. M.; Maitra, U. Supramolecular Gels: Functions and Uses. Chem. Soc. Rev. 2005, 34 (10), 821-836.

(31) Escuder, B.; Miravet, J. F. Functional Molecular Gels; Royal Society of Chemistry, 2013; Vol. 1.

(32) Terech, P.; Weiss, R. G. Low Molecular Mass Gelators of Organic Liquids and the Properties of Their Gels. Chem. Rev. 1997, 97 (8), 3133-3160.

(33) Khan, M. K.; Sundararajan, P. Encapsulation of Dye Molecules and Nanoparticles in Hollow Organogel Fibers of a Nonchiral Polyurethane Model Compound. Chem. Eur. J. 2011, 17 (4), 1184-1192.

(34) Khanna, S.; Khan, M. K.; Sundararajan, P. Influence of Double Hydrogen Bonds and Alkyl Chains on the Gelation of Nonchiral Polyurethane Model Compounds: Sheets, Eaves Trough, Tubes and Oriented Fibers. Langmuir 2009, 25 (22), 1318313193.

(35) Goodbrand, B.; Boils, D. C.; Sundararajan, P. R.; Wong, R. W. US Patent 6,414,051, 2002.

(36) Moniruzzaman, M.; Sundararajan, P. R. Low Molecular Weight Organogels Based 
on Long-Chain Carbamates. Langmuir 2005, 21 (9), 3802-3807.

(37) van Esch, J.; Kellogg, R. M.; Feringa, B. L. Di-Urea Compounds as Gelators for Organic Solvents. Tetrahedron Lett. 1997, 38 (2), 281-284.

(38) Khanna, S.; Moniruzzaman, M.; Sundararajan, P. R. Influence of Single versus Double Hydrogen-Bonding Motif on the Crystallization and Morphology of SelfAssembling Carbamates with Alkyl Side Chains: Model System for Polyurethanes. J. Phys. Chem. B 2006, 110 (31), 15251-15260.

(39) Helfrich, W.; Prost, J. Intrinsic Bending Force in Anisotropic Membranes Made of Chiral Molecules. Phys. Rev. A 1988, 38 (6), 3065.

(40) Guenet, J.-M. Polymer-Solvent Molecular Compounds; Elsevier, 2010. 
CHAPTER 5

\section{Self-Sorting, Sergeant-Soldier and Majority Rules Effects of Blends of Non-Chiral Biscarbamates Gels}




\section{CHAPTER V: SELF-SORTING, SERGEANT-SOLDIER AND MAJORITY RULES EFFECTS OF BLENDS OF NON-CHIRAL BISCARBAMATES GELS}

\subsection{Introduction}

We previously produced and characterized biscarbamate molecules with an even and odd number of carbon atoms in the side chain (also known as even biscarbamates and odd biscarbamates respectively). ${ }^{1,2}$ These molecules by themselves have a low melt viscosity of about 8-12 centipoises, a narrow melting temperature range $\left(\mathrm{T}_{\mathrm{m}}\right)$, and rapid crystallization upon quenching from the melt, all of which are ideal for inkjet printing. ${ }^{3}$ It was found that the viscosity and $T_{m}$ are varied by the choice of length of the alkyl side chains. It was also observed that melting a blend of two biscarbamates with different size side chains, both with the same distance between carboxylic groups, is not miscible. ${ }^{4}$ Biscarbamates, despite the identical distance between the hydrogen bonds and only a small difference in the number of carbons on the side chain, display the ability to self-sort, and this behaviour is more pronounced when the difference between the lengths of the side chains is large.

It was discovered that the control of the self-assembly of the biscarbamates can play a vital role in developing new classes of low molecular weight organic gelators (LMOGs). Gels can be easily transformed into solutions by heating them past their gel-to-solution temperature ( $\left.\mathrm{T}_{\text {gel-sol }}\right)$ and back to gels by cooling the solutions below their sol-gel transition temperature $\left(\mathrm{T}_{\mathrm{gel}}\right) .^{5}$ This reversible property of the gels can be used to make matrices for applications. ${ }^{6-11}$ A low concentration is sufficient for gel formation, and network filaments (length up to few micrometers) are formed by well-ordered arrays of molecules. Gelator 
molecules self-assemble by highly specific non-covalent interactions, leading to fibrous structures which form a 3D network. Therefore, self-complementary and unidirectional intermolecular interactions can be considered a prerequisite for the gelation ability. ${ }^{5,12}$ The intermolecular interactions between the organogelator molecules can be hydrogen bonding, London dispersion forces, electrostatic attractions, and $\pi$-stacking.

Gels of biscarbamates with even and odd number of carbon atoms in the side chains, at concentration of $0.08 \mathrm{M}$ in benzonitrile, are characterized in a previous paper ${ }^{13}$ and in Chapter 4. These gels are referred to as odd and even gels. It was found that the even gels formed short fibres. The shape of the fibres varied from flat fibres to bent sheets to hollow cylinders to full cylinders depending on the length of the side chains of the biscarbamate. ${ }^{13}$ Odd gels would form similar full cylinders or bent sheets, but they are in general much longer in length. In another experiment, a mixture of gels was produced by mixing an even biscarbamate side chain length $\left(\mathrm{C}_{8}\right)$ with an odd one $\left(\mathrm{C}_{9}\right)$ and then forming the gel. In this case, the gel formed long narrow fibres contrary to those found in the even side chain length only gels. This appears to be a case of a non-chiral sergeant-soldier system. It is for this reason that there is an interest in looking at the properties of blends of biscarbamate gels as well as the influence that certain biscarbamates have on other molecules. It is surprising that the biscarbamate blends showed self-sorting during crystallization, while the gelation of a blend would follow the sergeant-soldier behaviour.

In this chapter, we will examine the characteristics of the biscarbamate gel blends which include odd-odd and odd-even mixtures. We will also look at cases where short biscarbamates (smaller than $\mathrm{C}_{6}$ ), which normally do not gel on their own, would from a gel when mixed with longer chain biscarbamate $\left(\mathrm{C}_{6}\right.$ and longer). We will show that this is 
indeed evidence of the sergeant-soldier effect as all thermal and morphological properties now resemble those of the longer chain biscarbamates. We also hypothesize that the key property in the determination of which biscarbamate plays the sergeant role is determined by the rate of gelation in the assembly of the fibres grown in solution. We will also look at blends which show the "majority rules" and self-sorting principle instead of sergeantsoldier. The blends exhibiting the "majority rules" behaviour show physical and morphological properties depending on the component with the higher concentration in the blend. Blends that self-sort, such as melt and solvent mixtures, show properties of both individual components.

\subsection{Experimental}

\subsubsection{Synthesis of the Biscarbamates}

The synthesis of biscarbamates of different alkyl side chain lengths was described by Goodbrand et al. ${ }^{14}$ This involves reacting 1,6-diisocyanatohexane $(0.1 \mathrm{~mol})$ with various alcohols $(0.2 \mathrm{~mol})$ ranging from 1-propanol to 1-octadecanol in presence of the catalyst 1,4-Diazabicyclo [2,2,2] octane (DABCO) in toluene. In this chapter, we designate the biscarbamates as $\mathrm{C}_{\mathrm{x}}$ where $\mathrm{x}$ denotes the number of carbon atoms in the alkyl side chains.

\subsubsection{Preparation of the Gels and Xerogels}

Similar to our previous work on organogels of mono-carbamates ${ }^{15}$ and biscarbamates with even number of carbon atoms in the side chain, ${ }^{13}$ benzonitrile was used 
for the experiments. Gels of concentration $0.08 \mathrm{M}$ (the minimum gelation concentration for the shortest biscarbamate that could gel, which will be explained later on) were prepared in capped vials to avoid evaporation of the solvent. Weighed amounts of biscarbamates of different alkyl side chain lengths were dissolved in requisite amount of benzonitrile at a temperature ranging from $80-100{ }^{\circ} \mathrm{C}$, resulting in clear solutions. Gels were made either by quenching the clear solution in an ice bath or by slowly cooling to room temperature by turning off the hot plate. The vials were turned upside down to check for gelation. If the samples were not visually separated and did not flow perceptibly then they were considered gels. All the gels were opaque. Blends of gels were prepared by keeping a constant total molarity in the solution while varying the ratio of the individual components. Hence, all the combination gels were kept at a concentration of $0.08 \mathrm{M}$ in benzonitrile, which is the minimum gelation concentration for $\mathrm{C}_{6}$ (the smallest biscarbamate capable to gel). A small sample of these gels were dried at room temperature for two days and then kept under vacuum for a few more days to form xerogels.

\subsubsection{Characterization of the Methods}

Thermal analysis was performed using a TA Instruments 2010 differential scanning calorimeter at a heating rate of $5{ }^{\circ} \mathrm{C}$ per minute. The calorimeter was calibrated for temperature and energy with an indium sample as a standard reference material. DSC traces were recorded with about 7-8 $\mathrm{mg}$ of xerogel in nitrogen atmosphere. Sample preparation for the wet gels involved putting $30 \mathrm{mg}$ of the wet sample in an aluminium pan $(50 \mu \mathrm{L})$ without the lid and let the sample sit in the fumehood for exactly 30 minutes. Afterwards, the lid was crimped to the pan while making sure no solvent or condensation could escape. Seeing as the temperature range of the DSC run did not go very high (maximum $100{ }^{\circ} \mathrm{C}$ ), 
deformation of the pan due to excess vapors would not alter our results. The error in the $\mathrm{T}_{\mathrm{m}}$ measurement was less than $1{ }^{\circ} \mathrm{C}$. The software used to analyze the thermographs is TA Instruments Universal Analysis 2000 (version 3.9A).

The microscope images were recorded on a Zeiss Axioplan polarized optical microscope (OM). Northern Eclipse (version 6.0) image processing software was used to capture the image. The microscope is equipped with a Linkam hot stage for variable temperature optical microscopy and controlled by a macro from the Northern Eclipse software.

SEM images of the xerogels were obtained using a JEOL JSM-6400 scanning electron microscope. They are then mounted on carbon tape and sputter coated with $80 / 20$ $\mathrm{Au} / \mathrm{Pd}$ alloy. SEM images were captured at an accelerating voltage ranging from 5 to 20 $\mathrm{kV}$. Vega TC software was used for the microscope manipulation and to capture the images.

\subsection{Low Molecular Weight Blends}

The effect of blending two homologous self-assembling molecules on the structure and the morphology of the crystals was discussed in Chapter 3. Blending two homologous self-assembling molecules, which differ only in the length of the alkyl side chain, leads to self-sorting between them for melt and solvent blends. ${ }^{4}$ In those cases, we observed that this self-sorting behaviour can be controlled by modifying the growth or precipitation rate of the biscarbamate crystals. Although the central part of the molecules is the same, the difference in the side chain length causes a disruption in the packing of the molecules and 
changes in the melting temperature and crystallinity. In addition to a reduction in the spherulite size, the rate of growth of the spherulites is also affected. We noticed some significant differences in behaviour in comparison to melt or solution blends. However, in all cases, the presence of one biscarbamate can affect the morphology of the other biscarbamate component.

An important property of biscarbamates is that not all can form gels; they require a minimum concentration as well as a certain amount of intermolecular forces to allow for directional growth in solution. For gels, any biscarbamate shorter than $\mathrm{C}_{6}$ were unable to gel by itself regardless of the concentration or the solvent used. However, when blended with a sufficient concentration of a longer biscarbamate, the blend would form a gel with the physical characteristics of the longer biscarbamate. This indicates a sergeant-soldier behaviour.

At concentration $0.04 / 0.04 \mathrm{M}$ (50/50 ratio of each component), shorter biscarbamates are able to gel only with long biscarbamates as seen through the phase diagram below (Figure 5.1). An interesting observation is that the amount of the longer component in the blends is lower than the critical gelation concentration when by itself (Table 5.1). For example, $\mathrm{C}_{9}$ requires a minimum concentration $\left(\mathrm{C}_{\mathrm{min}}\right)$ of $0.06 \mathrm{M}$ by itself in gelling from benzonitrile. However, a $0.04 / 0.04 \mathrm{M}$ blend of $\mathrm{C}_{3}$ and $\mathrm{C}_{9}$ does indeed form a stable gel despite neither of the components would gel by themselves at that concentration. This suggests that the shorter component of these blends does indeed contribute to the gelation process and the gels are not merely a result of the gelation of the longer component. However, these gel blends are a lot less stable than the gels made with the longer component by itself. After the formation of the gels, a small amount of impact 
onto the vials, such as a hit onto the counter surface, can cause the gel to break. The gel blends also have a shorter shelf-life as they stay stable for up to a month whereas the individual gels would stay stable for a few months.

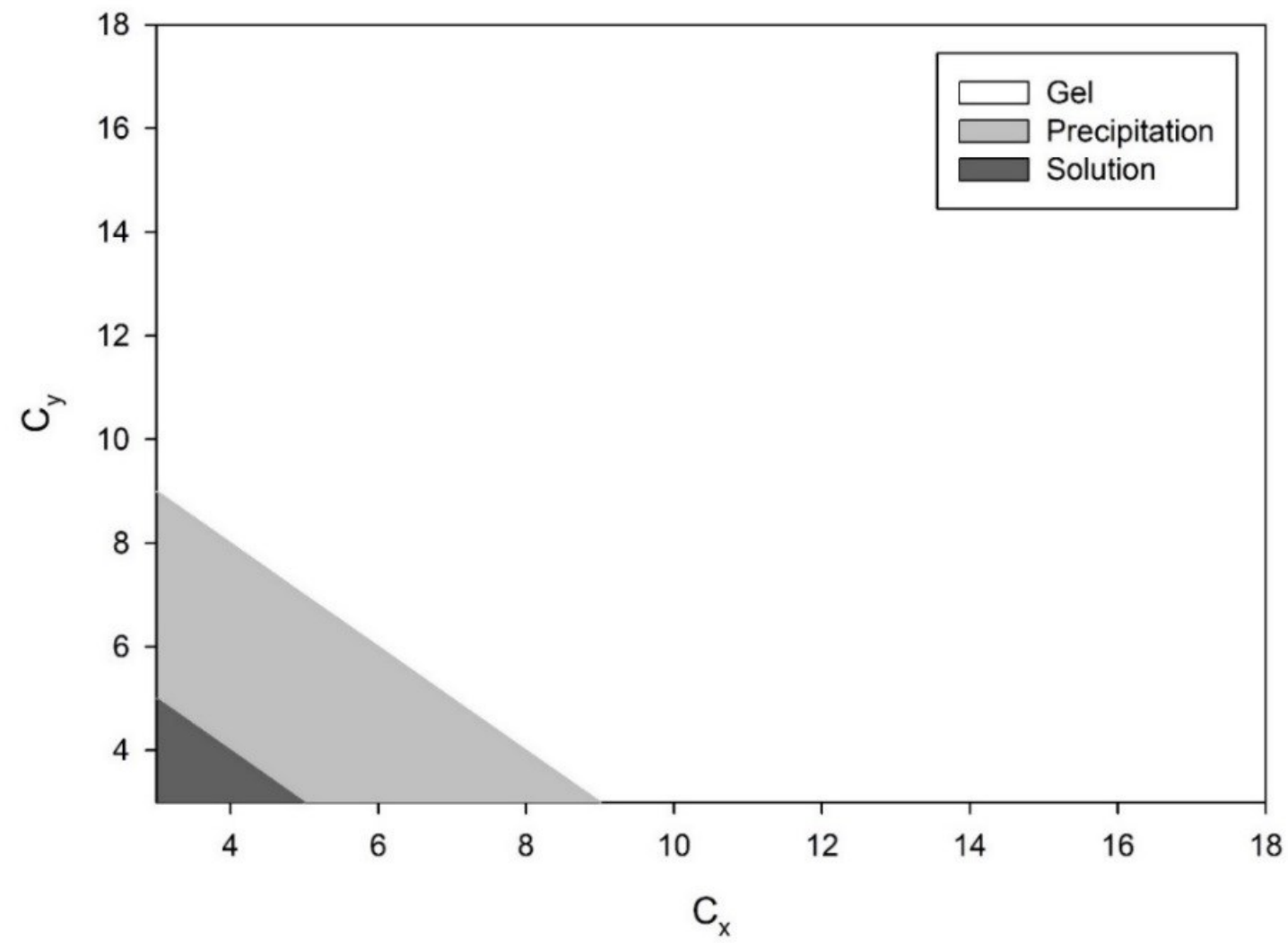

Figure 5.1. Phase diagram of biscarbamate blends at $50 / 50 \mathrm{C}_{\mathrm{x}} / \mathrm{C}_{\mathrm{y}}$ in benzonitrile. Shorter biscarbamate require longer biscarbamate in order to for a gel blend. 
Table 5.1. The critical gelation concentration for biscarbamate gels in benzonitrile.

\begin{tabular}{|c|c|}
\hline Biscarbamate & Concentration (M) \\
\hline $\mathrm{C}_{3}$ & N.A. \\
\hline $\mathrm{C}_{4}$ & N.A. \\
\hline $\mathrm{C}_{5}$ & N.A. \\
\hline $\mathrm{C}_{6}$ & 0.080 \\
\hline $\mathrm{C}_{7}$ & 0.064 \\
\hline $\mathrm{C}_{8}$ & 0.064 \\
\hline $\mathrm{C}_{9}$ & 0.064 \\
\hline $\mathrm{C}_{10}$ & 0.060 \\
\hline $\mathrm{C}_{11}$ & 0.040 \\
\hline $\mathrm{C}_{12}$ & 0.024 \\
\hline $\mathrm{C}_{13}$ & 0.020 \\
\hline $\mathrm{C}_{15}$ & 0.016 \\
\hline $\mathrm{C}_{16}$ & 0.016 \\
\hline $\mathrm{C}_{18}$ & 0.016 \\
\hline
\end{tabular}

N.B. For gels, any biscarbamates shorter than $\mathrm{C}_{6}$ were unable to gel by themselves no matter the concentration used. They either form a solution or precipitate out.

The influence on the presence of the shorter biscarbamate can also be seen from the decrease in the gel-to-solution temperature $\left(\mathrm{T}_{\text {gel }}\right)$ recorded using the DSC (Figure 5.2) and 
listed in Table 5.2. This change occurs after the addition of the shorter biscarbamate while keeping the concentration of the longer component constant. This suggests the ability to produce gels where the gel-to-solution temperature can be controlled by an addition of a short biscarbamate in the mixture. When analysing their thermal properties using DSC, these types of gels blends produce a single endotherm and a single exotherm representing the gel-to-solution and gelation temperatures of these gels respectively. These changes in physical state are repeated throughout many cycles of heating and cooling which shows reversibility in these gel blends (Figure 5.3).

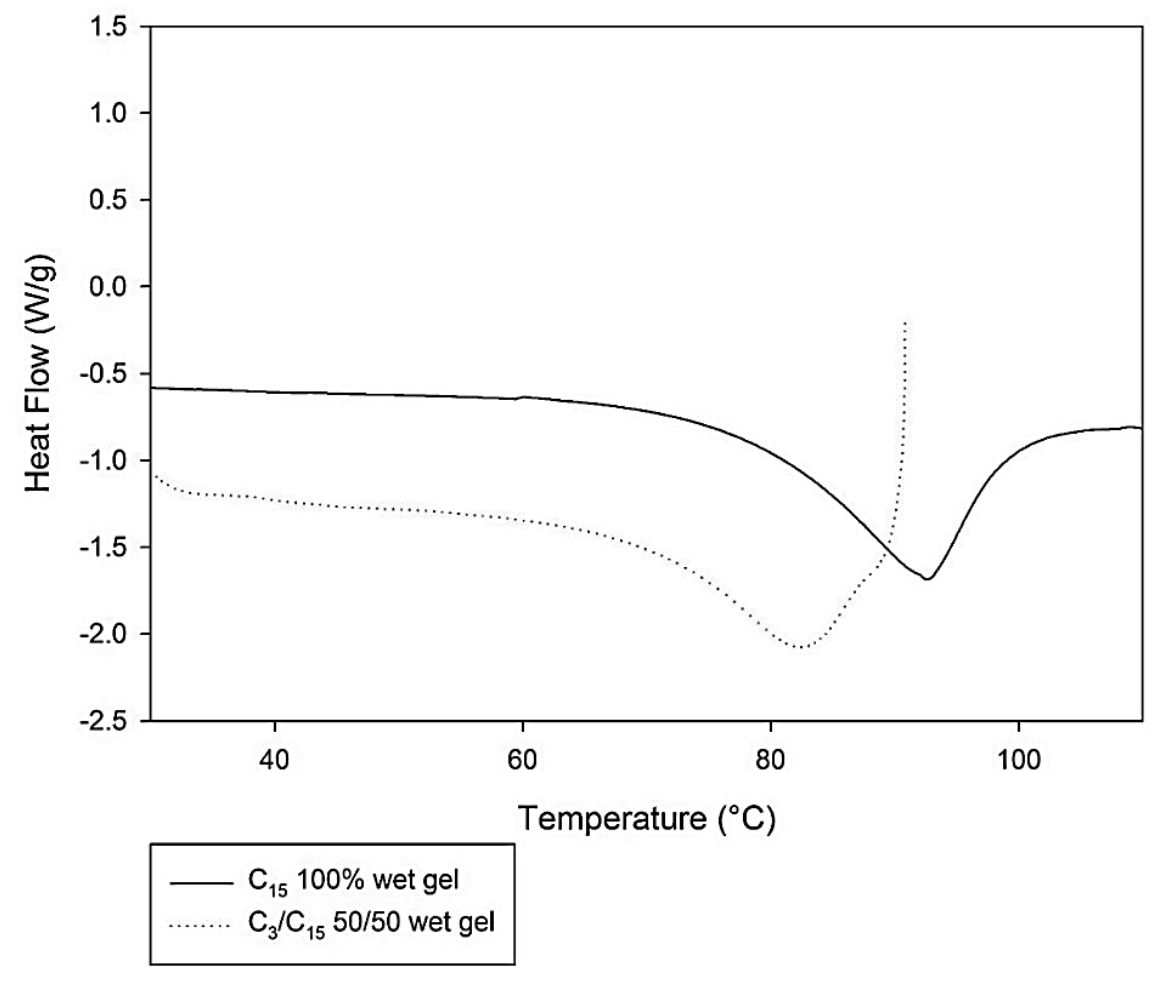

Figure 5.2. Endothermic peaks of biscarbamate gels of $C_{15}(0.04 \mathrm{M})$ and $\mathrm{C}_{3} / \mathrm{C}_{15} 50 / 50$ $(0.04 / 0.04 \mathrm{M})$ mixture in benzonitrile. There is a shift due to the presence of $\mathrm{C}_{3} . \mathrm{C}_{3}$ is incapable of gelling by itself even at 4 times the concentration. 
Table 5.2. The gel-to-solution temperature ( $\left.T_{\text {gel-sol }}\right)$ of short biscarbamate blend gels and their individual components with recorded using the DSC.

\begin{tabular}{lll}
\hline Biscarbamate & Concentration & Gel-Sol Temperature $\left({ }^{\circ} \mathrm{C}\right)$ \\
\hline $\mathrm{C}_{3}$ & $100 / 0$ & N.A \\
$\mathrm{C}_{3} / \mathrm{C}_{9}$ & $50 / 50$ & 52.0 \\
$\mathrm{C}_{4}$ & $100 / 0$ & N.A \\
$\mathrm{C}_{4} / \mathrm{C}_{8}$ & $50 / 50$ & 48.8 \\
$\mathrm{C}_{5}$ & $100 / 0$ & $\mathrm{~N} . \mathrm{A}$ \\
$\mathrm{C}_{5} / \mathrm{C}_{7}$ & $50 / 50$ & 42.7 \\
$\mathrm{C}_{6}$ & $100 / 0$ & 40.2 \\
$\mathrm{C}_{7}$ & $100 / 0$ & 52.9 \\
$\mathrm{C}_{8}$ & $100 / 0$ & 51.6 \\
$\mathrm{C}_{9}$ & $100 / 0$ & 62.2 \\
\hline
\end{tabular}




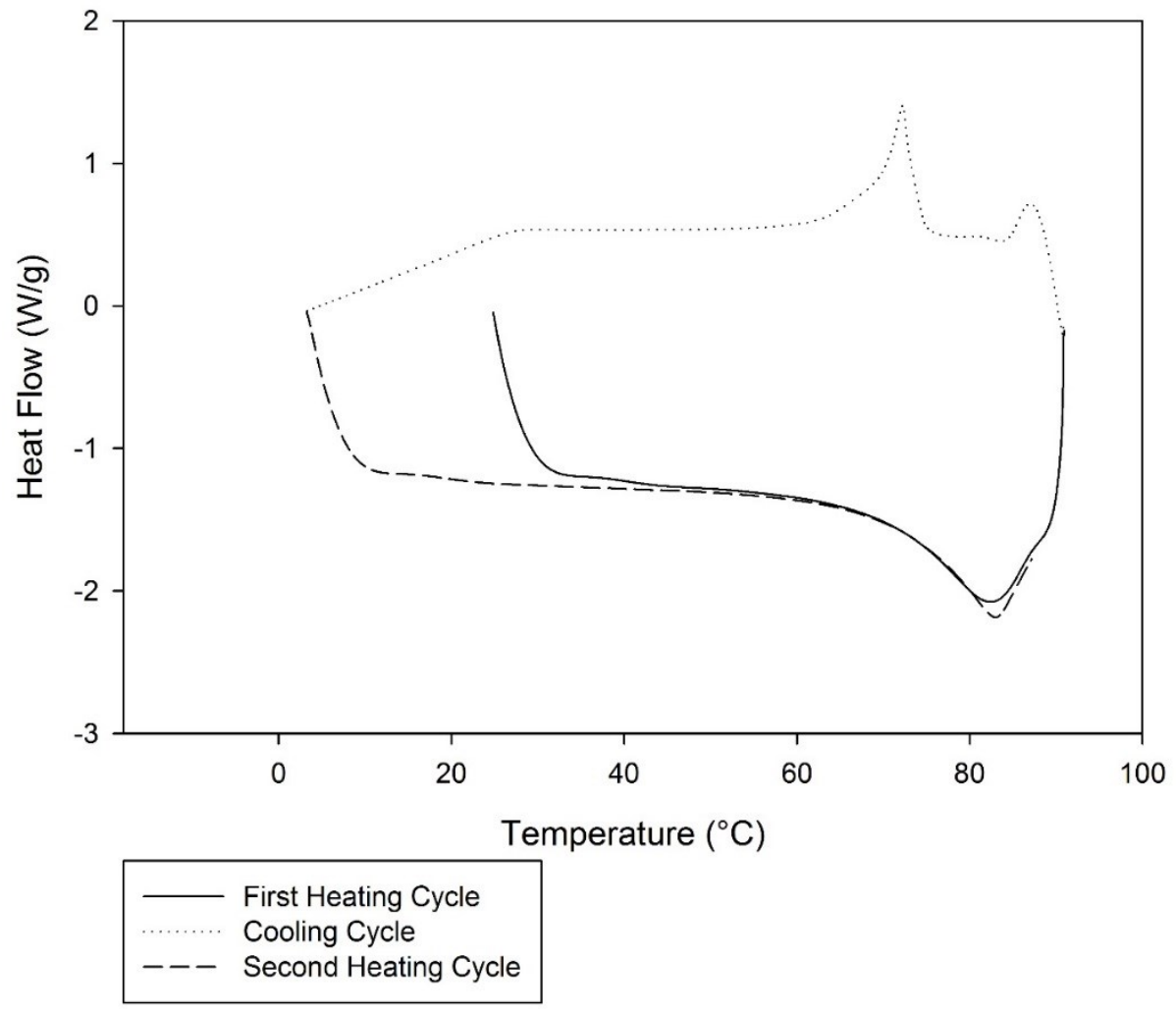

Figure 5.3. DSC thermographs of the gel-sol and sol gel transitions of $50 / 50 \mathrm{C}_{3} / \mathrm{C}_{15}$ showing thermoreversibility.

We looked further into these short biscarbamates mixed with longer biscarbamates blends. An interesting observation is the $\mathrm{C}_{3} / \mathrm{C}_{15}$ sample managed to form a gel and the lengths of the ribbons are significantly different depending on the method of preparation: either slow-cooling or quenching the solution in an ice bath (Figure 5.4). This might have to do with controlling the rate of crystallization or precipitation from the solvent and thus mutually affecting the size of the growth. We've seen this phenomenon in the solvent mixing sample. It would seem that this is again true for the gels as the growth based on 
self-assembly is basically the same only with solvent trapped in the fibre network. It is also interesting that this particular sample would gel at all considering $\mathrm{C}_{3}$ would not gel on its own. It thus requires another component to promote its gelation.
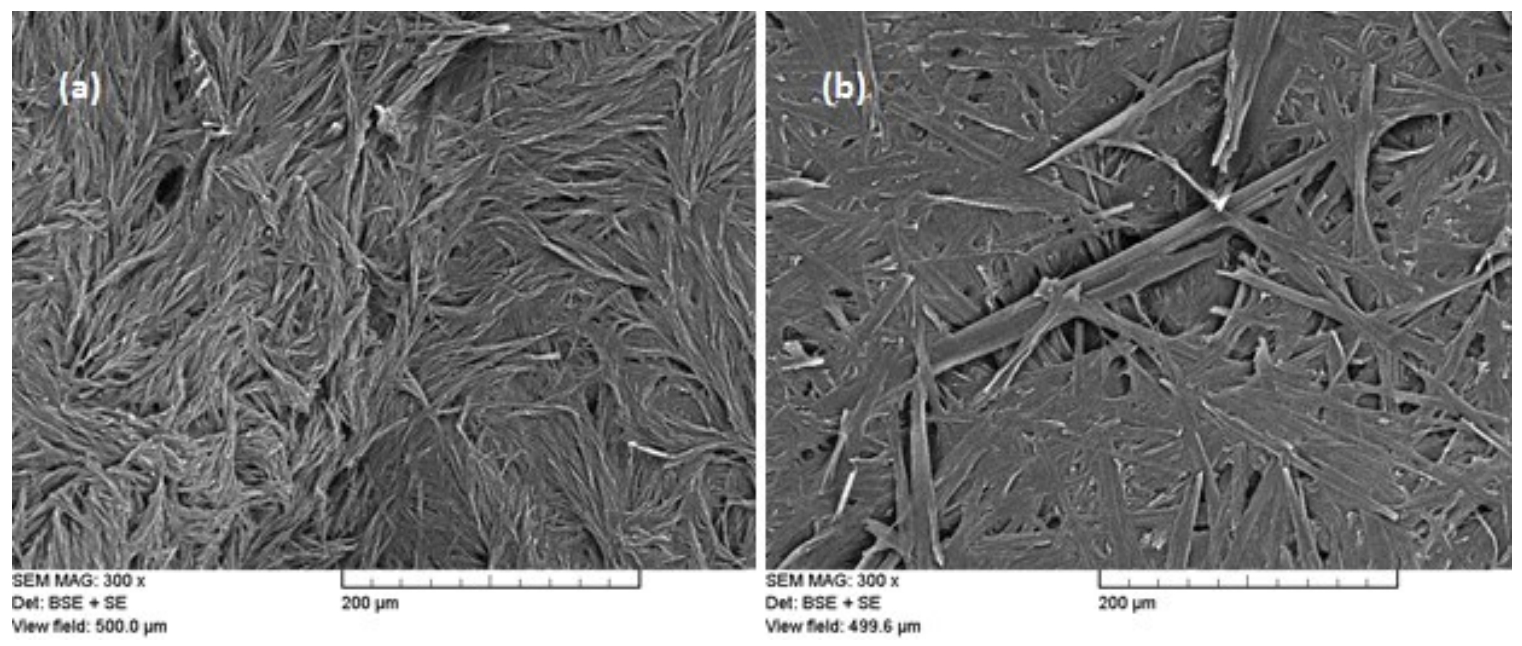

Figure 5.4. SEM images of xerogels of biscarbamate combination $50 / 50 \mathrm{C}_{3} / \mathrm{C}_{15}$ prepared by (a) quenched and (b) slow-cooled methods.

In the case of the slow-cooled $\mathrm{C}_{3} / \mathrm{C}_{15}$ blends, the fibres resemble those of the $\mathrm{C}_{15}$ only gels of the slow-cooled sample. However, for the quenched sample, the fibres show more narrow ribbon structures. The rapid formation of nucleation sites due to quenching would force the longer biscarbamate to assemble first. However, in this example, the sample does not look like the $\mathrm{C}_{15}$ biscarbamate only gel. This phenomenon is also seen for every short/long quenched biscarbamate gel combination. This suggests that the assembly of the fibres in quenched samples still depends on the presence of the shorter biscarbamate molecules. As the quenched $\mathrm{C}_{15}$ only gel resembled its slow-cooled counterpart, this 
indicates that the growth of the gel blend fibres produced by quenching is affected by the presence of the shorter biscarbamate.

In a solution blend, these types of biscarbamates would tend towards a self-sorting behaviour when rapidly cooled after dissolution. For the gels, if both components of the gel blend did indeed self-sort, we would see the $\mathrm{C}_{15}$ fibres as well as $\mathrm{C}_{3}$ precipitates in solution. However, this does not appear to be the case as seen in the optical microscope images. We did not observe the $C_{3}$ along with the $C_{15}$ gel fibres (Figure 5.5). Figure 5.5a shows that $\mathrm{C}_{3}$ biscarbamate forms clusters of small crystals in benzonitrile. In contrast, $\mathrm{C}_{15}$ biscarbamate form fine long gel fibres of over $100 \mu \mathrm{m}$ long as seen in Figure 5.5b. In the gel blend where the $\mathrm{C}_{3}$ biscarbamate component is at high concentration $(80 / 20)$, the resulting fibres resembling those of the $\mathrm{C}_{15}$ only gel. Despite being the major component in the blend, $\mathrm{C}_{3}$ biscarbamate crystals were not found in the blend. SEM images revealed similar findings where only fibres are found. This is an indication that the shorter component does not affect the morphology of the gel despite having an influence on the gelation process as seen by the difference in thermal properties. 


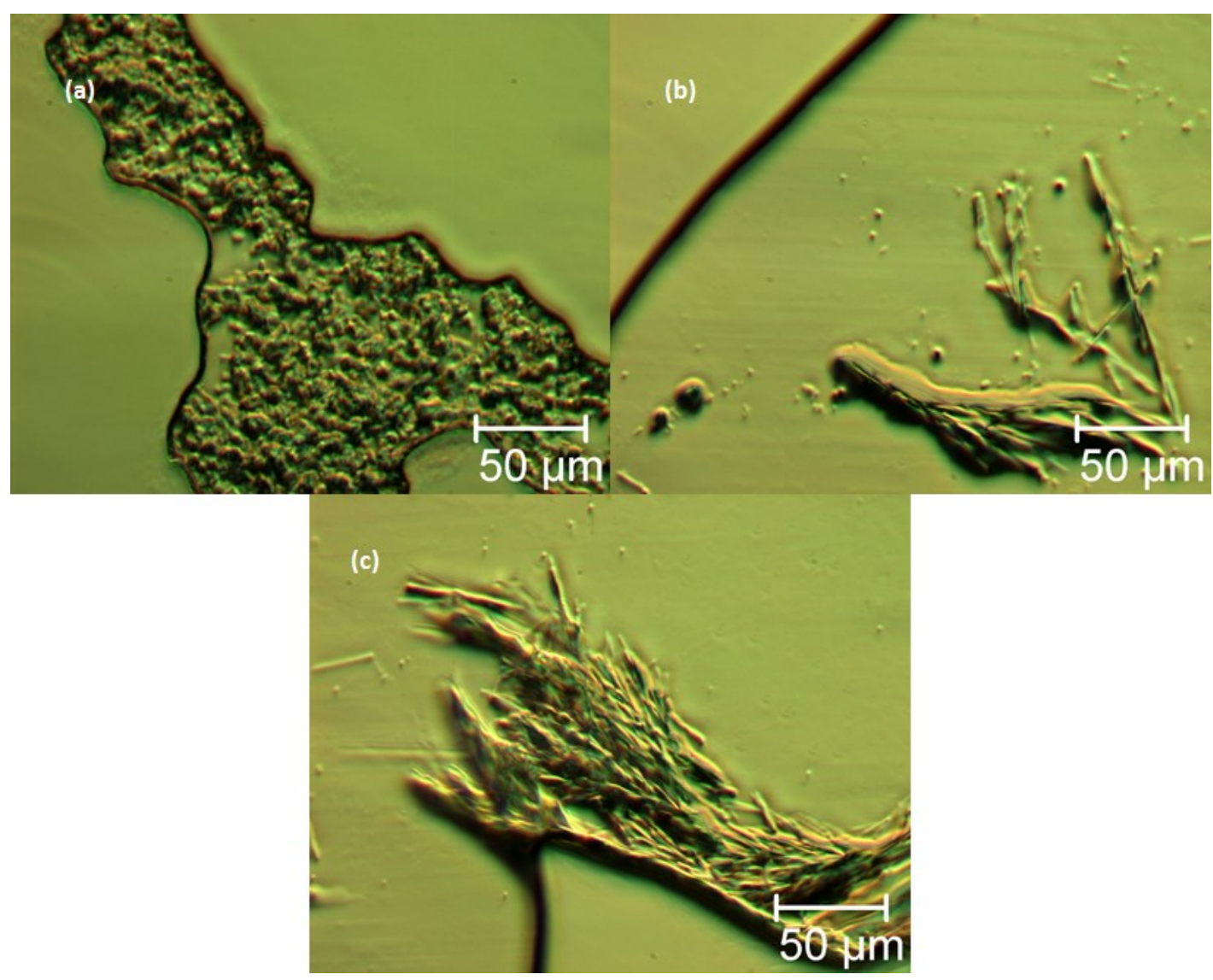

Figure 5.5. Optical micrographs of (a) $C_{3}$ in benzonitrile, (b) $C_{15}$ gel and (c) $C_{3} / C_{15} 80 / 20$ gel blend.

\subsection{Special Case of $\mathrm{C}_{8}$ and $\mathrm{C}_{9}$ Biscarbamate Gel Blends}

When we look at a blend of two biscarbamate that are very close to each other $\left(\mathrm{C}_{8}\right.$ and $\mathrm{C}_{9}$ ), we observe a clear sergeant-soldier phenomenon. In this case, an even biscarbamate is mixed with an odd biscarbamate where the difference in the number of carbon on the side chain is one. The fibres grown displayed a long narrow filled ribbons which is similar to the morphology of the $\mathrm{C}_{9}$ only gels (Figure 5.6). The blends do not show unwrapped short fibres resembling the $\mathrm{C}_{8}$ only gel. In addition, when the concentration of 
$\mathrm{C}_{9}$ decreases to only $10 \%$, the blends still resemble to those of the $\mathrm{C}_{9}$ only gel. This indicates that only a small amount of $\mathrm{C}_{9}$ is required to alter the morphology which is a clear case of sergeant-soldier phenomenon.

Optical microscope images were taken of the wet gel biscarbamate blend (Figure 5.7). The results are similar to those of the SEM. We did not observe any large disparities in fibre length for the $\mathrm{C}_{8} / \mathrm{C}_{9}$ combination despite obtaining the gel by slow-cooling or quenching. We also observe the similarities from both preparation methods for melt blends of biscarbamate. This differs from the $\mathrm{C}_{3} / \mathrm{C}_{15}$ blend where there are difference in morphology depending on the cooling method used. This may indicate that methods or gel preparation does not matter if both components are able to gel by themselves. Since $\mathrm{C}_{3}$ is not able to form a gel, the short biscarbamate molecules in solution could have affect the longer component during the self-assembly process when forming the gel. 

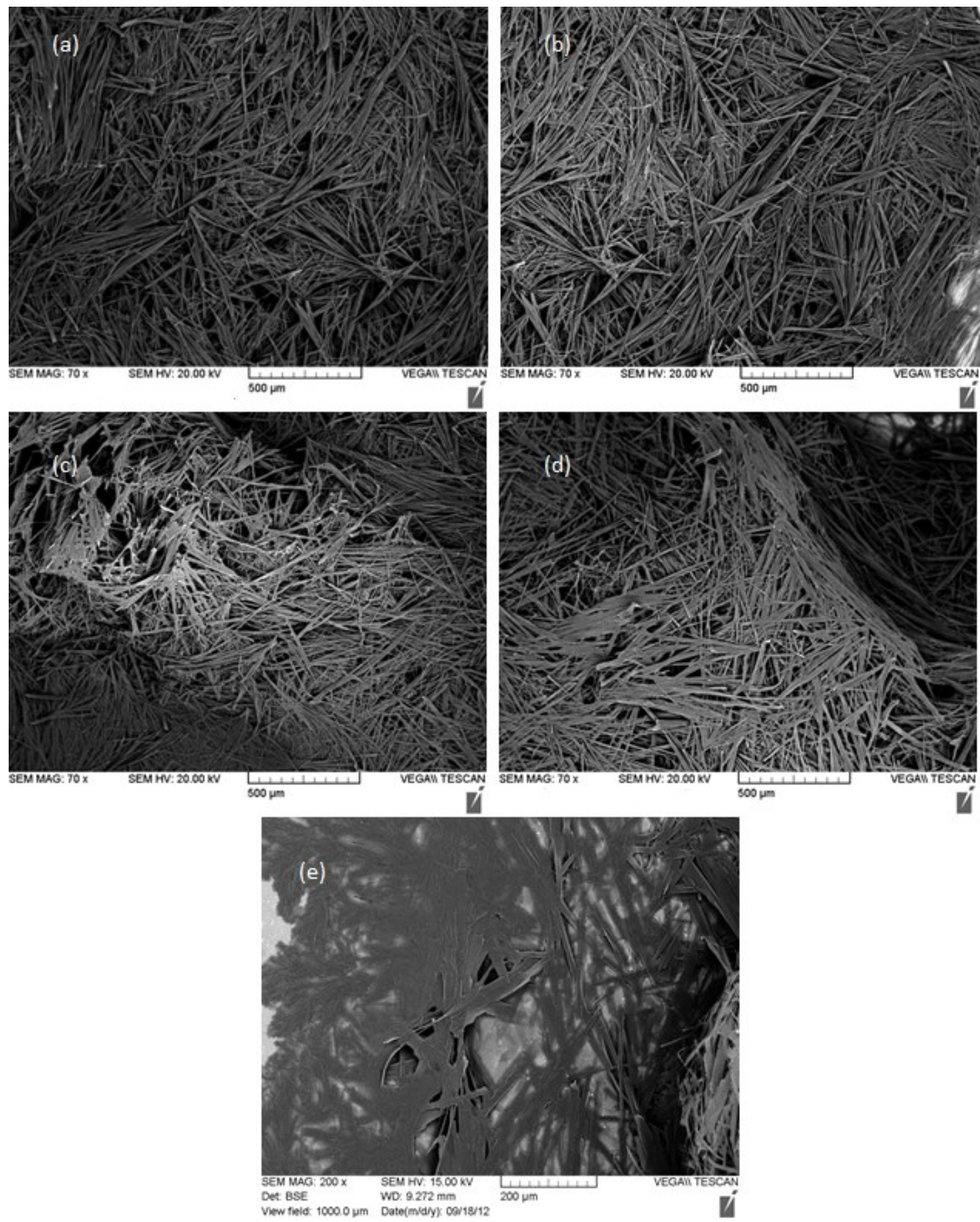

Figure 5.6. SEM images of xerogels of biscarbamate combination $\mathrm{C}_{8} / \mathrm{C}_{9}$ at concentration ratios of (a) $0 / 100$, (b) 50/50, (c) 80/20, (d) 90/10, and (e) 100/0. The difference in the magnification of the $\mathrm{C}_{8}$ only gel is to illustrate the unwrapped short fibres. Even at a closer magnification, the $\mathrm{C}_{8}$ only gel fibres take up less distance. 


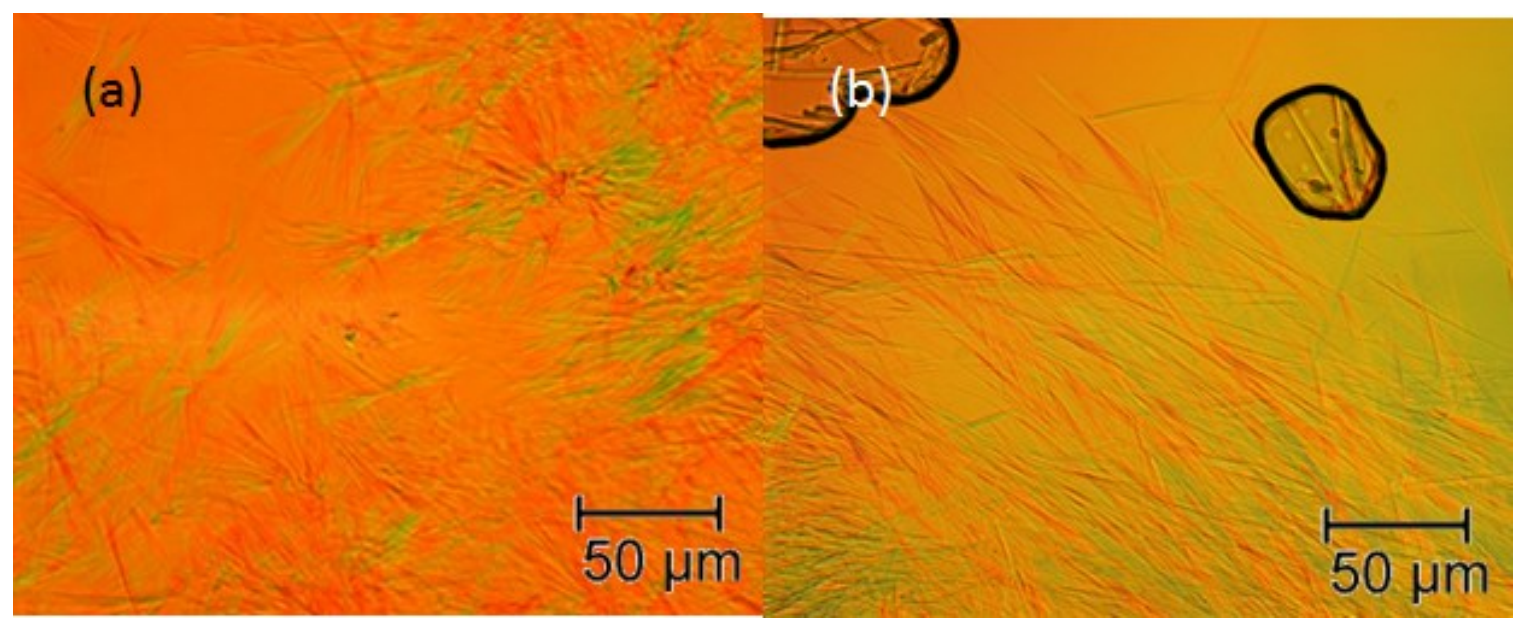

Figure 5.7. Optical microscope images of wet gels of biscarbamate 50/50 combination of $\mathrm{C}_{8} / \mathrm{C}_{9}$ prepared by (a) quenched and (b) slow-cooled methods.

Additionally, we looked at the thermal properties of the $\mathrm{C}_{8} / \mathrm{C}_{9}$ gel blends. Only one endotherm and one exotherm appear when running calorimetry. The values remained constant throughout multiple cycles suggesting that the gel blend is reversible. The values are listed in Table 5.3. The $\mathrm{T}_{\text {gel-sol }}$ values of the blends also lie in between those of the individual components. This illustrates proper miscibility without any indication of mutual diluent effect as seen in solution or melt blends. 
Table 5.3. The gel-to-solution temperature ( $\mathrm{T}_{\text {gel-sol }}$ ) of $\mathrm{C}_{8} / \mathrm{C}_{9}$ gel blends at various concentrations recorded using the DSC.

\begin{tabular}{ll}
\hline Concentration & Gel-Sol Temperature $\left({ }^{\circ} \mathrm{C}\right)$ \\
\hline $100 / 0$ & 51.6 \\
$90 / 10$ & 54.9 \\
$80 / 20$ & 57.3 \\
$50 / 50$ & 60.5 \\
$0 / 100$ & 62.2 \\
\hline
\end{tabular}

In Chapter 3, we looked at melt and solvent biscarbamate blends. We rationalized that these blends are the two biscarbamates are acting as mutual diluents ${ }^{16,17}$ (plasticizers) i.e., as eutectic mixtures and lowering each of the melting temperatures. We also discussed the influence of the length of the alkyl chain on the rates of crystallization and spherulite growth of these biscarbamates from the melt in a previous publication. ${ }^{1,2}$ In the case of the $\mathrm{C}_{8} / \mathrm{C}_{9}$ gel blends, $\mathrm{T}_{\text {gel-sol }}$ do not follow the same trend as the melt and solvent blends. The gel-to-solution temperatures of the blends all lie in between the $\mathrm{T}_{\text {gel-sol }}$ of the individual components. There is no mutual diluent effect, but instead a pure miscible mixture. We propose that the rate of fibre formation is crucial to the sergeant-soldier phenomenon and to this sample's miscibility. In the previous chapter, the time required for gel formation of each biscarbamate was illustrated in Figure 4.2. The times required for a slow-cooled sample of $0.08 \mathrm{M} \mathrm{C}_{8}$ and $\mathrm{C}_{9}$ biscarbamate to gel are 28.5 and 26.5 minutes respectively. In the melt and solvent blends, we were able to show that biscarbamates with rate of 
crystallization that are close together produced more miscible blends. This seems to be the case for gel formation as well. The trend of the $\mathrm{T}_{\text {gel-sol }}$ shows it is following a miscibility curve. However, since the time of gelation for $\mathrm{C}_{9}$ biscarbamate is slightly lower, this would indicate that the $\mathrm{C}_{9}$ molecules would drive the assembly for the rest of the blend. This was observed for the case of $\mathrm{C}_{3} / \mathrm{C}_{15}$ and every other low molecular weight gel blend.

\subsection{Biscarbamate Gels Blends with a Difference of Four $\mathrm{CH}_{2}$ Groups in the Side- Chain Length}

For the gel blends with a difference of two $\mathrm{CH}_{2}$ groups in side chain length $(\Delta \mathrm{x}=$ 4), we used a combination of two odd biscarbamates of intermediate lengths: $\mathrm{C}_{7} / \mathrm{C}_{11}$ gel (Figure 5.8 and 5.9). Unlike the $\mathrm{C}_{3} / \mathrm{C}_{15}$ blend, the shape and the size of the fibres for the slow-cooled and quenched samples are similar. In addition, these fibres resemble those of the $\mathrm{C}_{11}$ alone gel in shape and dimensions. This might be indication of a sergeant-soldier effect where $\mathrm{C}_{11}$ grows long fibres as well. However, when we increase the concentration of the $\mathrm{C}_{7}$ component, the fibres began to decrease in length and started to resemble a more dense aggregation of fibres similar to $C_{7}$ only gels (Figure 5.10). When looking up the details of the fibres, the fibres of the $\mathrm{C}_{7}$ only gel appear aggregated, short and thick. As the addition of $\mathrm{C}_{11}$ molecules increases, the fibres become more detached and well formed. They also decrease in thickness while increasing in length. This combination seemed to display a majority rules behaviour in which the component at the larger concentration dictates the morphology in which the blend will take. Indeed, at higher $\mathrm{C}_{11}$ concentrations, the gel resembles $\mathrm{C}_{11}$ only fibres as displayed in Figure 5.10. 


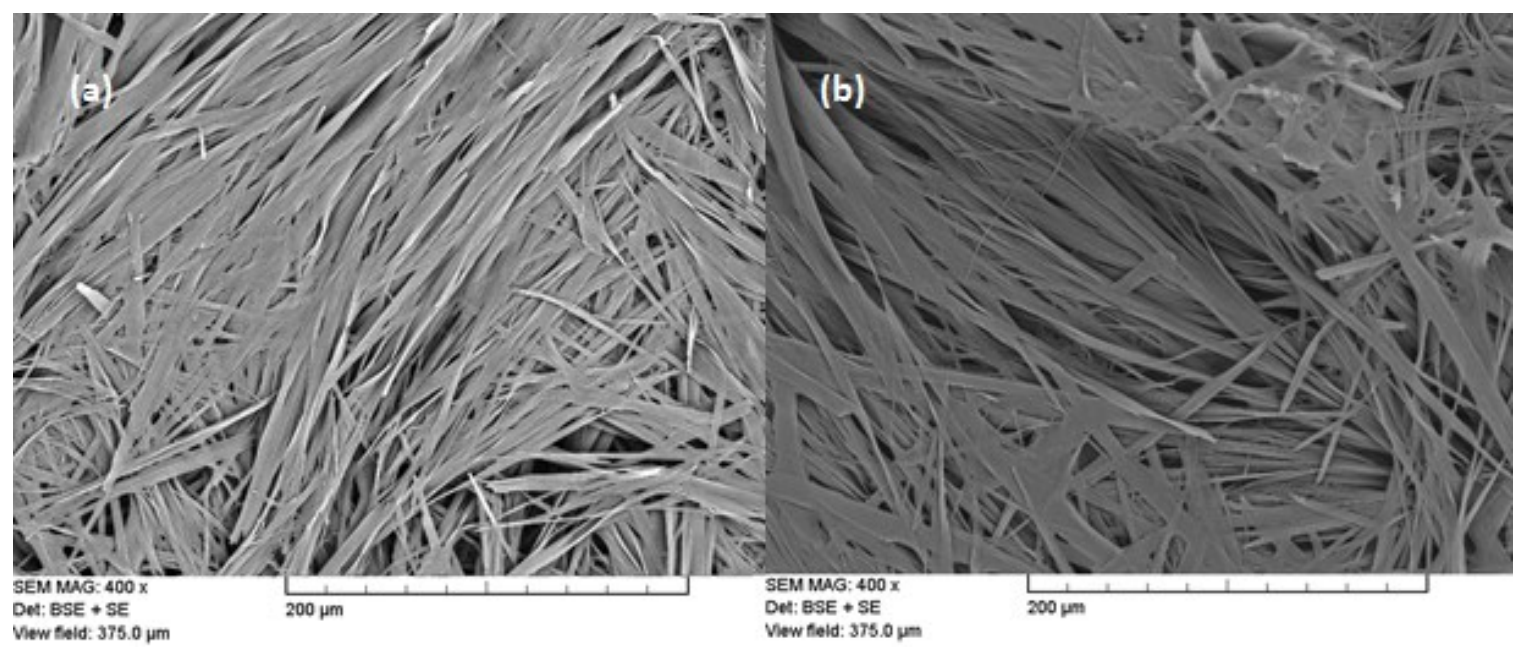

Figure 5.8. SEM images of wet gels of biscarbamate combinations $50 / 50 \mathrm{C}_{7} / \mathrm{C}_{11}$ prepared by quenched (a) and slow-cooled (b) methods.

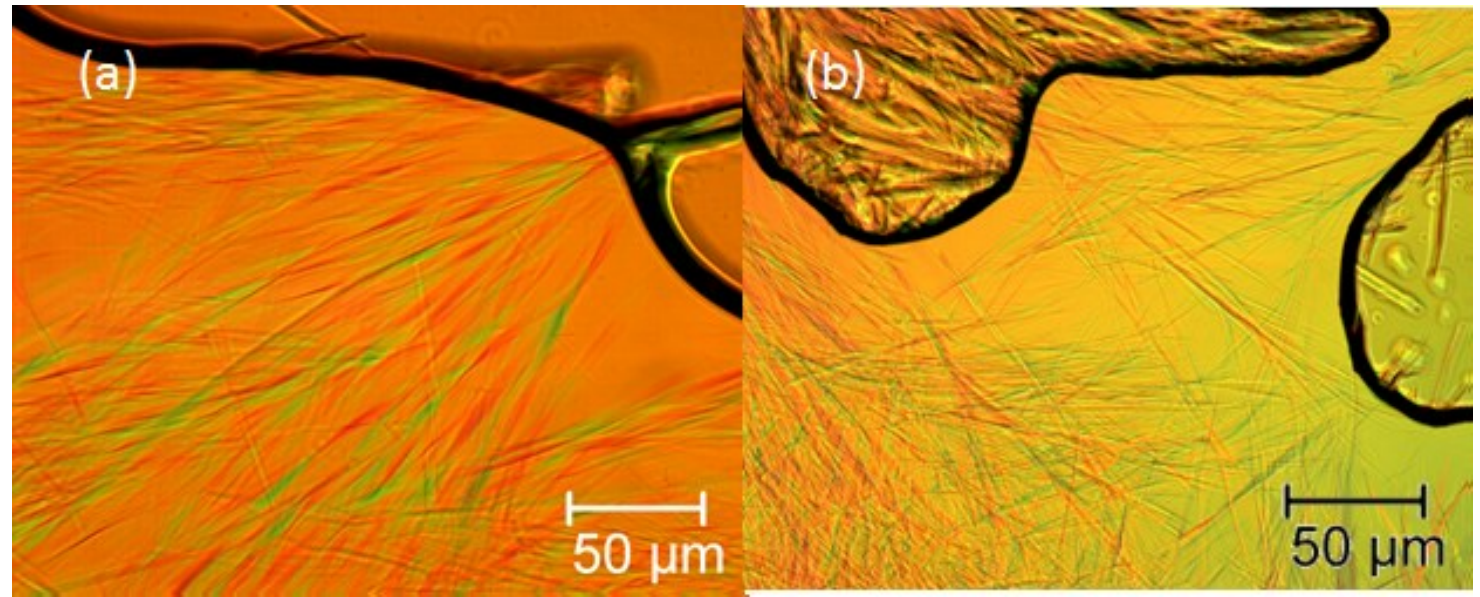

Figure 5.9. Optical microscope images of wet gels of biscarbamate 50/50 combination of $\mathrm{C}_{7} / \mathrm{C}_{11}$ prepared by (a) quenched and (b) slow-cooled methods. 


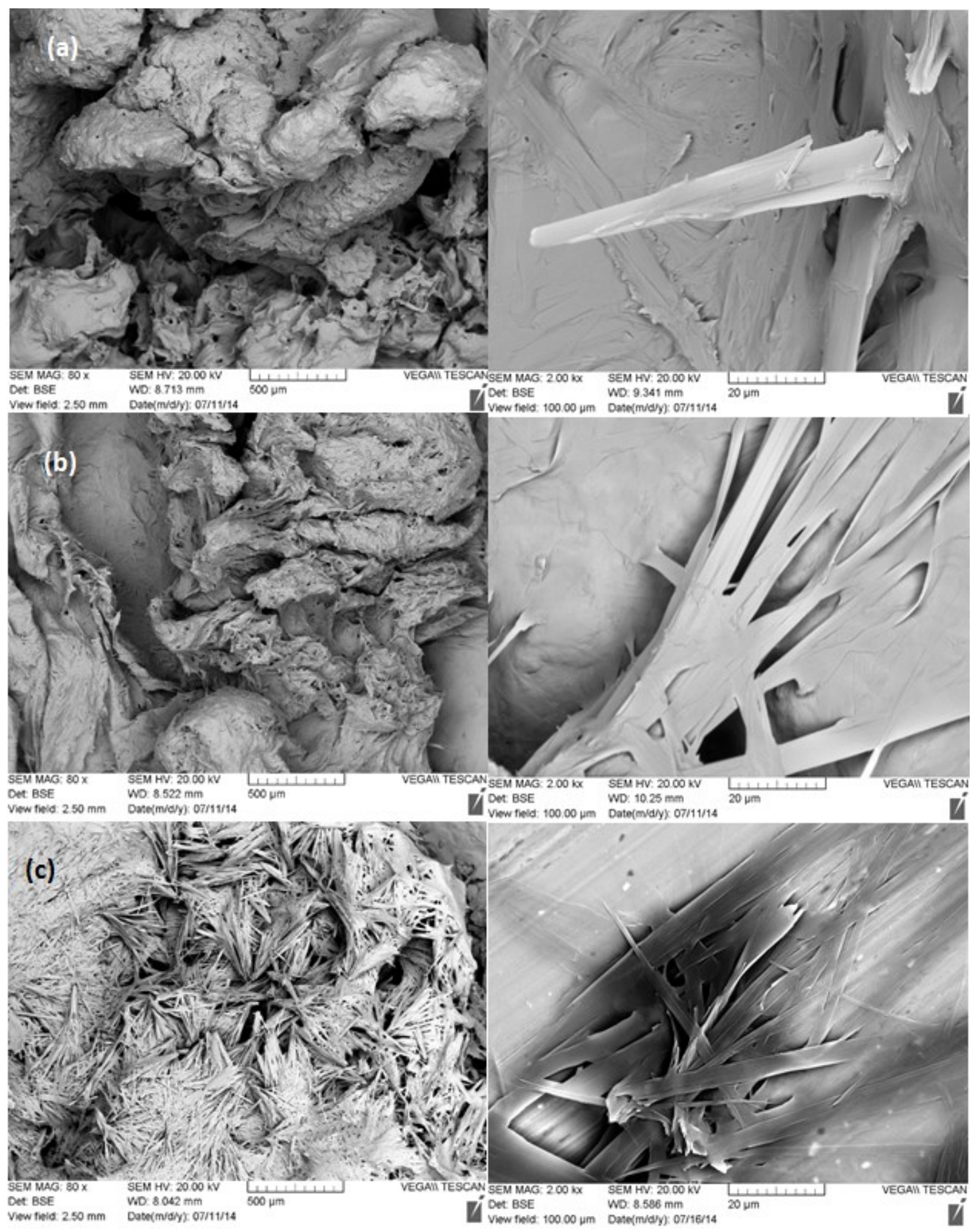

(Figure 5.10 cont'd on next page) 


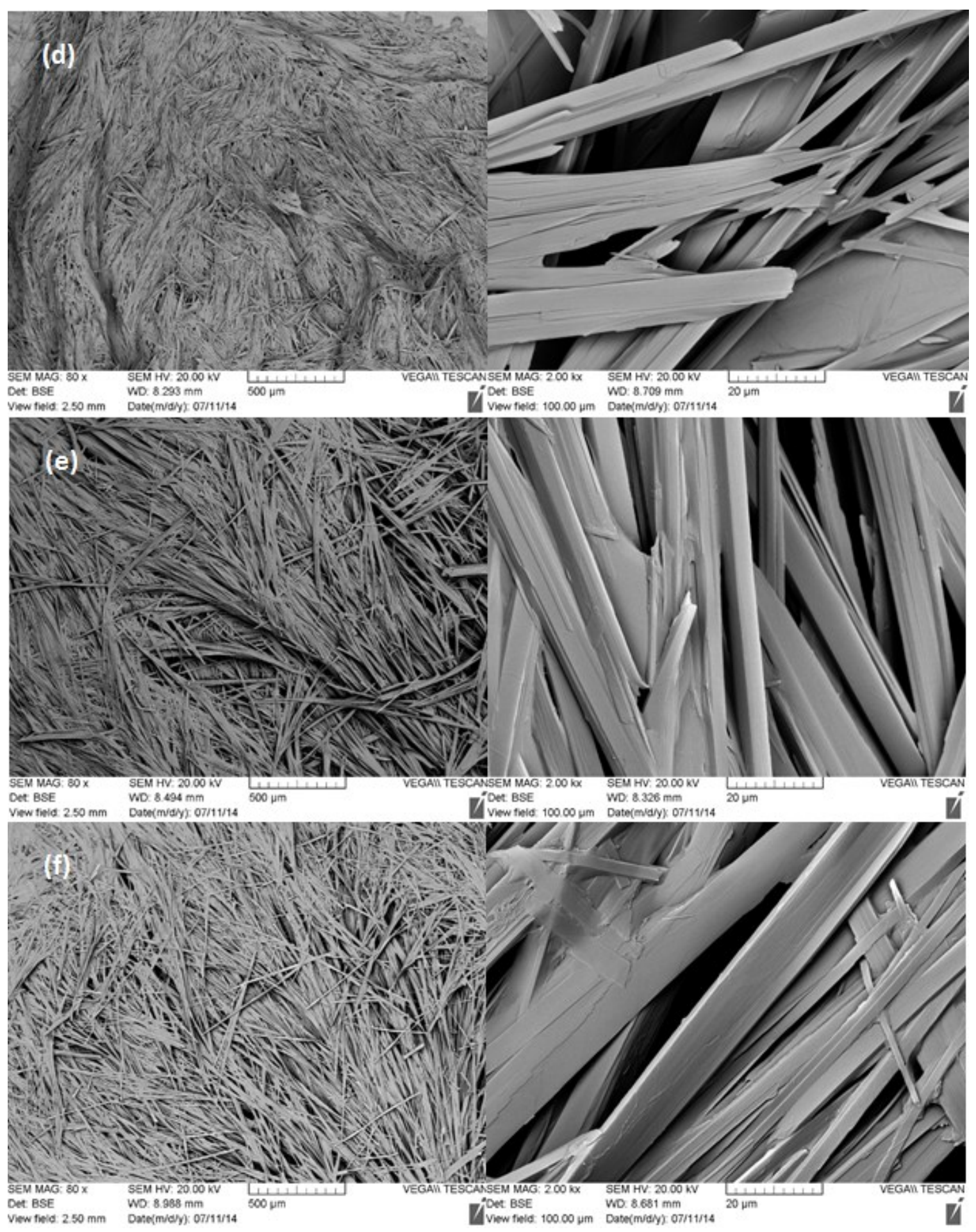

(Figure 5.10 cont'd on next page) 


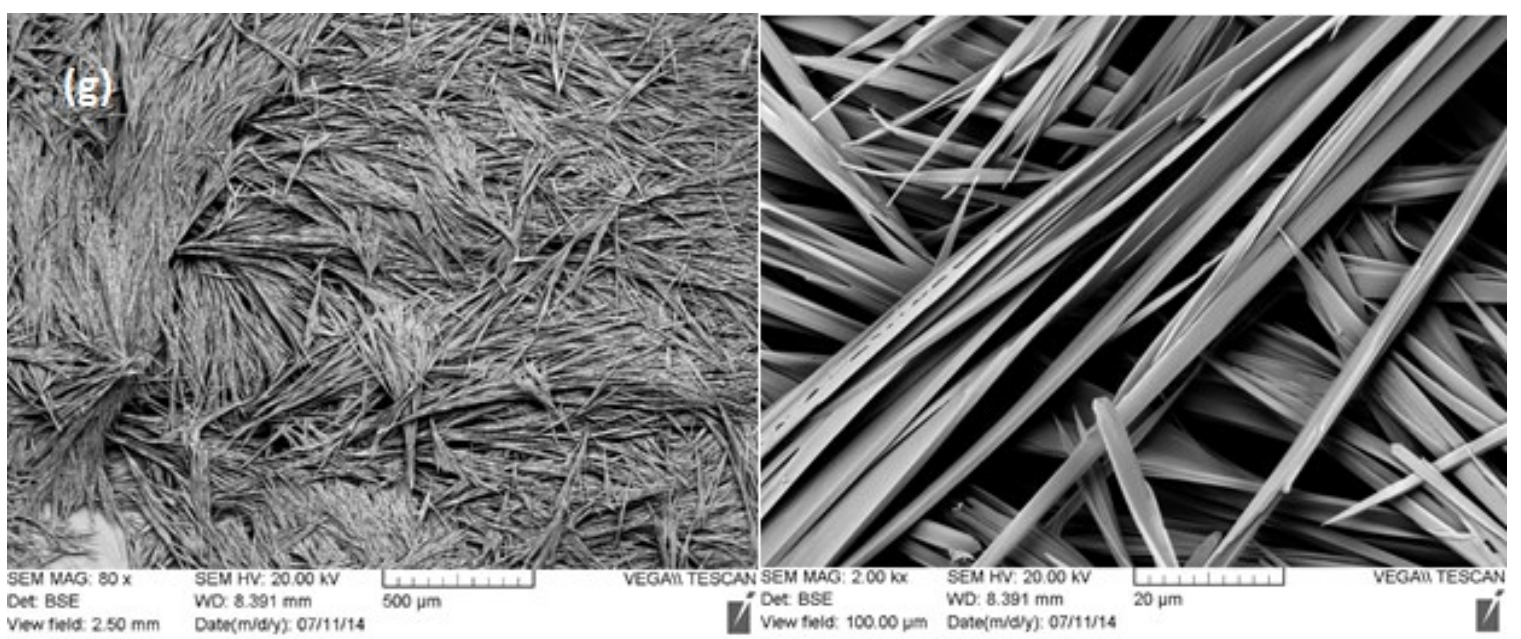

Figure 5.10. Low and high resolution SEM images of xerogels of biscarbamate combination $\mathrm{C}_{7} / \mathrm{C}_{11}$ at concentration ratios of (a) 100/0, (b) $90 / 10$, (c) $80 / 20$, (d) 50/50, (e) 20/80. (f) 10/90, and (g) 0/100.

In regards to the thermal properties of these blends, the gels displayed two distinctive $\mathrm{T}_{\text {gel-to-sol }}$ and $\mathrm{T}_{\text {gel }}$ when the sample was examined using the DSC (Figure 5.11). In addition, these distinctive multiple peaks are reproducible through multiple heating and cooling cycles. The thermal properties suggest that, much like the melt and solution based blends previously studied, the components in the gel based blend are not fully miscible. In fact, these results seem to indicate the formation of two separate gels. However, this was not observed by microscopy where it shows that the more concentrated component would dictate the morphology by leading the self-assembly process. 


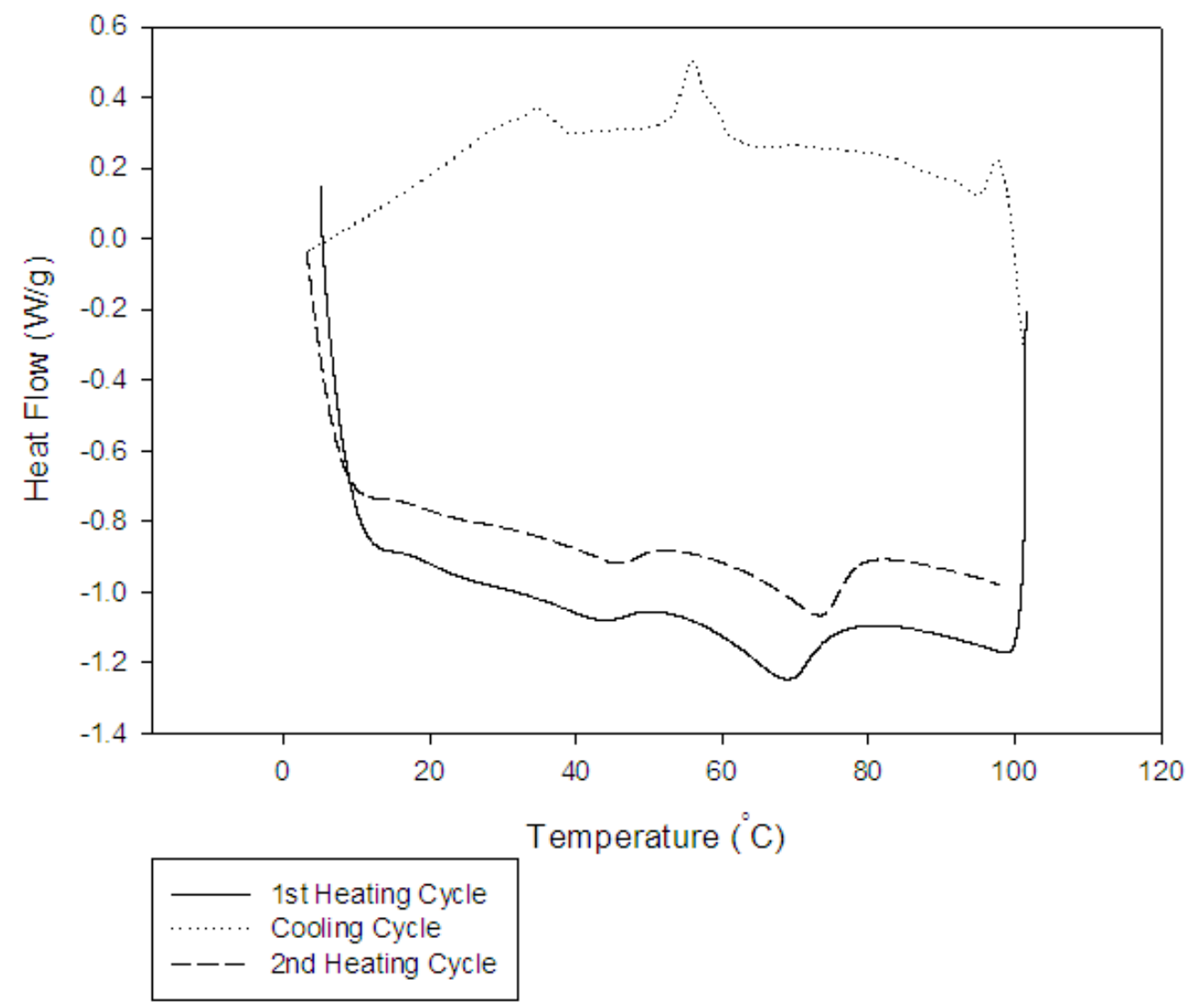

Figure 5.11. DSC thermographs showing the gel-sol, sol gel transitions of $\mathrm{C}_{7} / \mathrm{C}_{11}: 50 / 50$. The individual peaks represent the endotherms of the individual component. It would appear that they are not miscible in the gels level.

Taking into account both experiments, we justify these two different observations by claiming that the majority-rules effect is a combination of both the self-sorting effect on the thermal properties and the concentration dependency of the individual components in the morphology. As the morphology of the blends resembles closer to that of the more concentrated component, the shape of the fibres are still slightly different. This difference 
is more perceptible as the concentration of both components are closer together. These types of fibre formation is the result of the domain mixing of the different biscarbamates as see in the melt and solvent blends. The more concentrated component will have a larger effect on the fibre morphology, and this effect is reduce when the concentrations becomes similar. This is in agreement to the thermograph where the $\mathrm{C}_{7}$ bis domains transitions at a lower temperature than the $\mathrm{C}_{11}$ bis domains.

\subsection{Gel Blends of Biscarbamates with a Difference of Two $\mathrm{CH}_{2}$ Groups in the Side- Chain Length}

Finally, we looked at a combination of closer related biscarbamate in terms of length. We looked at the odd-odd combination where the difference in side chain lengths is of two (2) carbons: $\mathrm{C}_{7} / \mathrm{C}_{9}$. In this case, neither the sergeant-soldier nor the majority rules behaviours were observed (Figure 5.12). SEM images show signs of multiple components: there are some long ribbon-like fibres mixed in a web of short and narrow string fibres. In fact, these mixtures of different types of fibres appear in both the slow-cooled and quenched gel blends. This may be explained by the self-sorting effect that was observed in some melt and solvent blends. 

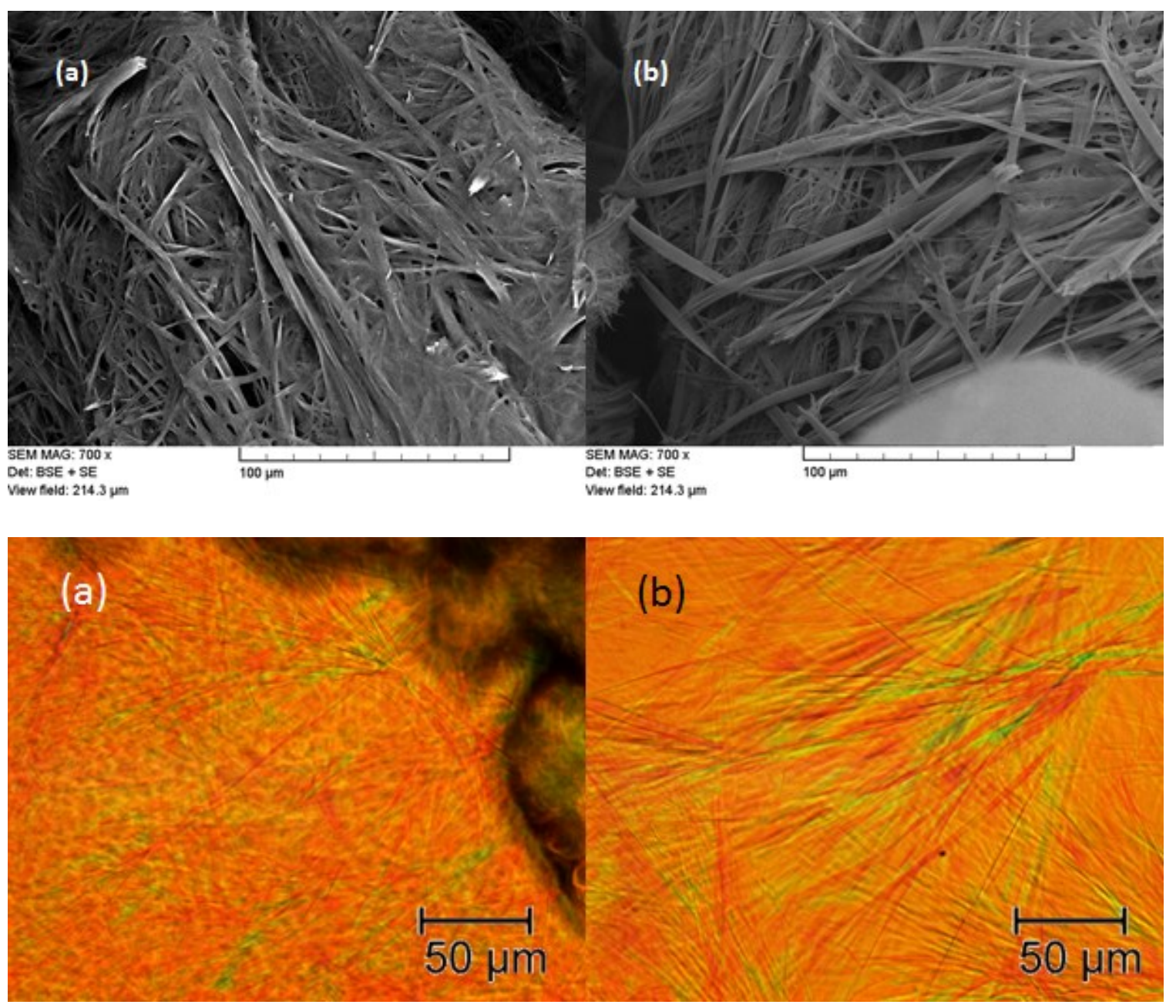

Figure 5.12. SEM and OM images of wet gels of biscarbamate combination $50 / 50 \mathrm{C}_{7} / \mathrm{C}_{9}$ prepared by quenched (left) and slow-cooled (right) methods.

We then looked at the miscibility of the gel mixtures. From observations of the melt and solvent-based blends, it was determined that the miscibility of the eutectic mixture depends on the rate of crystallization. When the self-sorting behaviour occurred, there was a large difference in the rate of crystallization between both components. The rates can be 
bridged to a certain extent in the presence of a solvent. In the case of gel blends, we looked into the gelation process as it is comparable to the process of precipitation in solution. ${ }^{18}$ We intend to study the behaviour and physical properties of these gel blends. We first looked at the thermal reversibility of these gels.

The thermograph produced from the DSC shown in Figure 5.13, shows that a gel blend of $50 / 50 \mathrm{C}_{7} / \mathrm{C}_{9}$ mixture is non-reversible. A single endotherm appears in the first heating cycle representing the first $\mathrm{T}_{\text {gel-to-sol }}$ temperature. The cooling cycle (at a rate of 5 ${ }^{\circ} \mathrm{C} /$ minute) shows each component gels individually. The next heating cycle clearly show the separation of both components. This is repeated for many cycles. The multiple endotherms for the following heating cycles do agree with the multiple components visualized in the SEM micrographs of the xerogels. As we see the different components isolated within parts of the gel blend, we would expect to have different $T_{\text {gel-to-sol. }}$ However, the single endotherm in the first cycle seemed a bit of an oddity. This may have to do with the initial forming of the gel. As these gel blends were left to cool slowly, once the gels had set, they would have had time to sit and perhaps go through some rearrangements. It is entirely possible that, while still having solution molecules not entrapped in the network, the assembly of the gel fibres can be rearranged since the $\mathrm{C}_{7}$ and $\mathrm{C}_{9}$ biscarbamate molecules are so closely related. The attractive forces of these molecules can still allow for mixing to occur to some extent. If the gels didn't have the same amount of time to rest, we would observe only the formation of the individual components such as we did in the next cycles in the DSC. This illustrates that the aging of the gel blends can affect the physical properties when the individual components are still so closely related. 


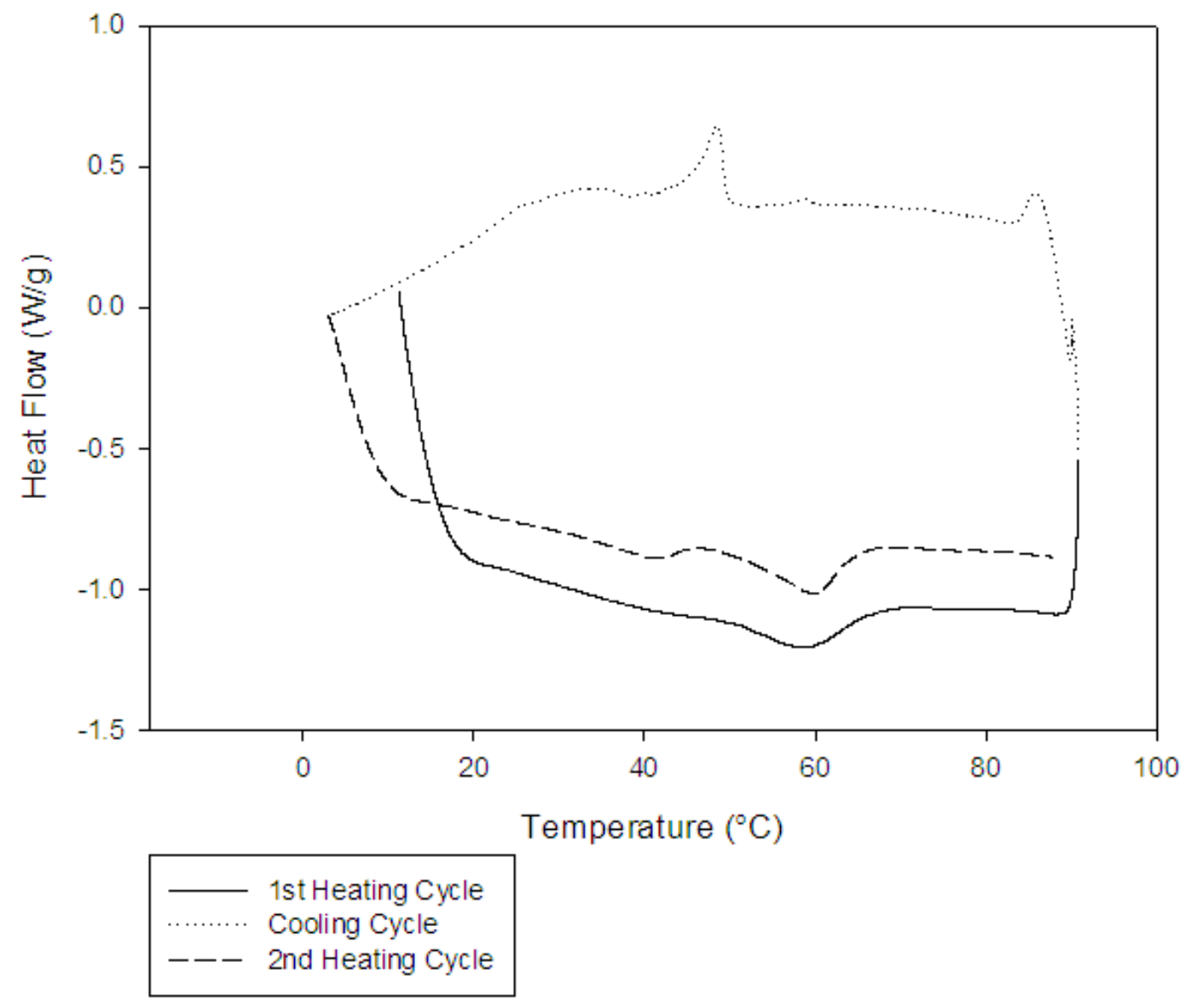

Figure 5.13. DSC thermographs showing the gel-sol, sol-gel transitions of $\mathrm{C}_{7} / \mathrm{C}_{9}$. This does not seem reversible as the first endotherm shows one peaks, and the ones in the following cycles show two peaks.

\subsection{Conclusions}

It was previously observed that melt and solvent-based blending of two molecules which are capable of hydrogen-bond-mediated self-assembly would often lead to selfsorting. It was believed that the key to miscibility is the crystallization rate and this can be 
controlled with the presence of a solvent. We then looked at biscarbamate gel blends; we studied their morphology and thermal properties. Three different blending behaviours were observed: sergeant-soldier, majority rules and self-sorting.

For any blends with biscarbamates containing less than six carbon groups along the side chain and that cannot gel on their own, the fibres resembles the longer biscarbamate component. This may be due to the fact that the shorter biscarbamate cannot grow on their own; hence, the resulting gels appear to have a sergeant-soldier type behaviour. Also, it appears that the $\mathrm{C}_{8} / \mathrm{C}_{9}$ gels also yield fibres that resembled the longer biscarbamate $\left(\mathrm{C}_{9}\right)$. As the concentration of the longer biscarbamate decreases, the resulting gel fibres still resemble the $\mathrm{C}_{9}$ only gels. These are the only two cases of the sergeant-soldier effect observed. The DSC showed a single endotherm and exotherm that are reproducible throughout many cycles for both cases. For any odd-odd gels blends with a difference in side chain length of $\Delta x=4$, such as $C_{7} / C_{11}$, we observed a majority rules effect. The fibre morphology is determined by the component with the higher concentration. In addition, there appears to have two endotherms which are reproducible throughout many cycles. Finally, for certain odd-odd biscarbamate mixtures where $\Delta x=2$ in the size of the chain, we observed a self-sorting effect. Both types of fibres are shown in the xerogel. The DSC also showed a single endotherm in the first cycle, but the following cycles showed multiple endotherm. A hypothesis for the change in thermal behaviour is that the allotted time to rest after the setting of the gel may have allowed the molecules to rearrange and form a more thermal miscible blend. During the heating and cooling cycles in the DSC, the gel 
did not have sufficient amount of time to allow for such rearrangements. The formation of the individual components were observed visually.

The results discussed above indicate that gel blends can yield a variety of different assembly behaviour. With the hydrogen bond and van der Walls forces mainly driving the biscarbamate fibres network formation, we observed just three different effects on the assembly. We do understand that the difference in side chain length of the individual component does determine what type of behaviour the gels would adopt. If it were possible to control self-assembly in this manner, it would have a great impact on the material industry. 


\subsection{References}

(1) Khan, M. K.; Sundararajan, P. R. Effects of Carbon Atom Parity and Alkyl Side Chain Length on the Crystallization and Morphology of Biscarbamates, A Set of Model Compounds for Polyurethanes. J. Phys. Chem. B 2011, 115 (27), 8696-8706.

(2) Khanna, S.; Moniruzzaman, M.; Sundararajan, P. R. Influence of Single versus Double Hydrogen-Bonding Motif on the Crystallization and Morphology of SelfAssembling Carbamates with Alkyl Side Chains: Model System for Polyurethanes. J. Phys. Chem. B 2006, 110 (31), 15251-15260.

(3) Goodbrand, H. B.; Boils, D. C.; Sundararajan, P. R.; Wong, R. W. Acoustic Printing Inks Containing Bis (carbamates). US Patent 6414051 B1 2002.

(4) Khan, M. K.; Sundararajan, P. R. Molecular Selectivity and Immiscibility during the Crystallization of Mixtures of a Set of Homologous Self-Assembling Molecules. $J$. Phys. Chem. B 2008, 112 (14), 4223-4232.

(5) Escuder, B.; Miravet, J. F. Functional Molecular Gels; Royal Society of Chemistry, 2013; Vol. 1.

(6) Arsenault, A. C.; Míguez, H.; Kitaev, V.; Ozin, G. A.; Manners, I. A Polychromic, Fast Response Metallopolymer Gel Photonic Crystal with Solvent and Redox Tunability: A Step towards Photonic Ink (P-Ink). Adv. Mater. 2003, 15 (6), 503507.

(7) Bailey, G. C.; Holm, V. C. F. Sulfate-Treated Zirconia-Gel Catalyst. US Patent 
3032599 A 1962.

(8) Buerkle, L. E.; Rowan, S. J. Supramolecular Gels Formed from Multi-Component Low Molecular Weight Species. Chem. Soc. Rev. 2012, 41 (18), 6089-6102.

(9) Kunze, W. A. Extended Release Hot and Cold Gel Fragrance Cartridges and Method of Making the Same. US Patent 5575992 A 1996.

(10) Peer, D.; Karp, J. M.; Hong, S.; Farokhzad, O. C.; Margalit, R.; Langer, R. Nanocarriers as an Emerging Platform for Cancer Therapy. Nat. Nanotechnol. 2007, $2(12), 751-760$.

(11) Sangeetha, N. M.; Maitra, U. Supramolecular Gels: Functions and Uses. Chem. Soc. Rev. 2005, 34 (10), 821-836.

(12) Terech, P.; Weiss, R. G. Low Molecular Mass Gelators of Organic Liquids and the Properties of Their Gels. Chem. Rev. 1997, 97 (8), 3133-3160.

(13) Khanna, S.; Khan, M. K.; Sundararajan, P. Influence of Double Hydrogen Bonds and Alkyl Chains on the Gelation of Nonchiral Polyurethane Model Compounds: Sheets, Eaves Trough, Tubes and Oriented Fibers. Langmuir 2009, 25 (22), 1318313193.

(14) Goodbrand, B.; Boils, D. C.; Sundararajan, P. R.; Wong, R. W. US Patent 6,414,051, 2002.

(15) Moniruzzaman, M.; Sundararajan, P. R. Low Molecular Weight Organogels Based 
on Long-Chain Carbamates. Langmuir 2005, 21 (9), 3802-3807.

(16) Vrentas, J. S.; Duda, J. L. Diffusion of Small Molecules in Amorphous Polymers. Macromolecules 1976, 9 (5), 785-790.

(17) Burke Jr, O. W.; Davis, P.; Kizer, J. A. A. Polymerization Process. Google Patents June 27, 1972.

(18) Estroff, L. A.; Hamilton, A. D. Water Gelation by Small Organic Molecules. Chem. Rev. 2004, 104 (3), 1201-1218. 


\section{CHAPTER 6}

\section{Biscarbamate Organogelation Using Microwave Heating}

Source 


\section{CHAPTER VI: BISCARBAMATE ORGANOGELATION USING MICROWAVE HEATING SOURCE}

\subsection{Introduction}

Non-conventional techniques such as microwave (MW) as the heat source, ionic liquids as an alternate medium, ultrasound, and ball-milling have been explored in the past few years for synthesis and processing of materials,. A tutorial review on these methods has been presented recently by Vázquez et al. ${ }^{1}$ Microwave-assisted organic synthesis was first demonstrated in the research labs of Giguere ${ }^{2}$ and Gedye. ${ }^{3}$ It was shown that the use of microwave dramatically accelerated the rate of many organic reactions compared to conventional conductive heat. This technique recognizes the ability of some materials to transform electromagnetic energy into heat. Since then, microwave heating has been applied not only to reduce reaction time $e^{2-5}$ but also to improve yields ${ }^{6,7}$ and selectivity in non-solvent. ${ }^{8,9}$ The process also allows reactions to occur with limited or absence of catalytic reagents which has great benefits in the field of synthetic chemistry. We have shown that in the case of curing of polymerizable monomers to fabricate a polyimide resins, the time to completion of curing was reduced to an hour, compared to more than $30 \mathrm{hrs}$ using the conventional heating. ${ }^{10}$ Nüchter $e t a l^{11}$ combined MW and mechano-chemistry for conducting organic reactions. Recently, Dudley et $a l^{12}$ and Stiegman et $a l^{13}$ presented a detailed discussion on the effect of the dipole moment of the solvent and solute and other parameters that affect the MW heating.

We have previously reported the organogelation with biscarbamates. ${ }^{14-16}$ Preparing the gels usually involves selecting a liquid that is a non-solvent for the molecule (gelator) 
at room temperature, but would dissolve upon heating to a higher temperature. Cooling the solution leads to the self -assembly of the molecule in the presence of the solvent, with predominant growth along the hydrogen bonding direction. ${ }^{14}$ Such directional growth results in fibres which trap the solvent molecules and gelation occurs. A minimum concentration of the gelator is required for gelation. In addition, the phenomenon also depends on the solute-solvent pair. In the absence of gelation, crystallization and precipitation would occur. While several studies on MW assisted synthesis and processing have been reported in the literature as mentioned above, we examined the effect of MW heating method on organogelation using the biscarbamates, with alkyl chain length ranging from $\mathrm{C}_{5}$ to $\mathrm{C}_{18}$.

In this chapter, we will discuss biscarbamate organogels using microwave heating and compared these gels to those produced by using conventional heating methods. It was observed that microwave heating had strongly affected the self-assembly of these gels as is illustrated by the morphology, the dissolution and the gel-to-solution temperatures, and the minimum critical gelation concentration $\left(\mathrm{C}_{\min }\right)$. This is the first reported experiment where the concept of self-assembly, particularly the production of gels, under microwave is examined. This may present a new and interesting alternative in the production of organogels.

\subsection{Experimental}

\subsubsection{Preparation of the Gels and Xerogels}

The synthesis of biscarbamates with different alkyl side chain lengths was 
described before. ${ }^{14-17}$ This involves reacting 1,6-diisocyanatohexane $(0.1 \mathrm{~mol})$ with various alcohols $(0.2 \mathrm{~mol})$ ranging from 1-propanol to 1 -octadecanol in presence of the catalyst 1,4-Diazabicyclo [2,2,2] octane (DABCO). As in our previous publications, we designate the biscarbamates as $\mathrm{C}_{\mathrm{x}}$ where $\mathrm{x}$ denotes the number of carbon atoms in the alkyl side chains.

As with our previous work, benzonitrile was used as the primarily solvent. Gels of concentration $0.08 \mathrm{M}$ were prepared in capped vials to avoid evaporation of the solvent. Gels were also prepared with DMSO, 1-octanol, xylene, toluene, and dodecane to study the effect of the solvent type. Conventional hot plate and microwave source were used for preparation of the gels. For the gels prepared using the hot plate, weighed amounts of biscarbamates of different alkyl side chain lengths were dissolved in requisite amount of solvent at around $80-100{ }^{\circ} \mathrm{C}$, which is about $20{ }^{\circ} \mathrm{C}$ above the dissolution temperature, resulting in clear solutions. This would ensure that the solution would be free of any remaining nuclei which promote gelation through a self-seeding process. Gels were made by slowly cooling to room temperature by turning off the hot plate. Vial inversion was used as a check for the gelation. The norm that is usually used is that when the vials were turned upside down and if the solute/solvent were not visually separated and did not flow they were considered as gels.

For the gels prepared using the microwave system, weighed amounts of biscarbamates of different alkyl side chain lengths and the requisite amount of solvent were placed in a $20 \mathrm{~mL}$ Pyrex vial. The vial was then placed in a porcelain vessel into an ETHOS PRO microwave digestive system running with the Milestone Digestion operating system. The samples were exposed to a maximum power of $1000 \mathrm{~W}$ of radiation as the software 
automatically delivers the minimum power required to follow the defined temperature profiles. The chamber containing the samples was programmed to set temperatures ranging from 40 to $160^{\circ} \mathrm{C}$ in 10 minutes and to keep that temperature for 30 minutes to two hours. The temperature of the chamber was measured using a temperature probe (ATC-400) inserted inside a reference vessel. This allows for the monitoring of the thermal conditions and adjusting the microwave parameters to achieve the desired temperature profile inside all the vessels. As the concentration and the solvents changed, the program temperature was modified to ascertain that the fully dissolved solutions were obtained prior to lowering the temperature for gelation. Due to visual limitations of the instrument, we were not able to determine the dissolution temperature visually. At the end of the entire heating and cooling process, the vials that do produce a uniform gel were considered to have an adequate dissolution temperature, while the samples which show precipitation or partial dissolution are determined to have insufficient heating. The radiation was then terminated and the samples were left to cool to room temperature at a rate of $1{ }^{\circ} \mathrm{C} /$ minute and left at that temperature for an hour. Once again, the vials were turned upside down to check for gelation. All the gels were opaque. These gels were dried at room temperature for a day and then kept under vacuum for a few days to form xerogels. There were no signs of degradation or chemical reactions of the biscarbamate molecules when using microwave heating.

\subsubsection{Characterization}

Thermal analysis was performed using a TA Instruments 2010 differential scanning calorimeter at a heating rate of $10^{\circ} \mathrm{C}$ per minute. This method allows for the measurement of the melting temperature of the gels: also known as the gel-to-solution temperature ( $\mathrm{T}_{\text {gel- }}$ 
sol). Differential scanning calorimetry also allows us to determine the formation of the gel i.e., the gelation temperature $\left(\mathrm{T}_{\text {gel }}\right)$. However, since the technique requires the sample to be heated in a conventional manner, acquiring $\mathrm{T}_{\text {gel }}$ for the microwave gels is not possible. The calorimeter was calibrated for temperature and energy with an indium sample as a standard reference material. Sample preparation for the wet gels involved putting $30 \mathrm{mg}$ of the wet sample in an aluminium pan $(50 \mu \mathrm{L})$ without the lid and let the sample sit in the fume hood for 10 minutes. The lid was then crimped to the pan while making sure no solvent or condensation would escape. Since the maximum temperature used for the DSC scans did not exceed $100^{\circ} \mathrm{C}$, deformation of the pan due to excess vapors would not alter our results. The difference in the recording temperature was less than $1{ }^{\circ} \mathrm{C}$. The software TA Instruments Universal Analysis 2000 (version 3.9A) was used to analyze the thermographs.

Optical micrographs $(\mathrm{OM})$ of the gels were recorded using a Zeiss Axioplan polarized optical microscope (POM) along with the Northern Eclipse (version 8.0) imageprocessing software.

SEM images of the xerogels were obtained using a JEOL JSM-6400 scanning electron microscope. They are then mounted on carbon tape and sputter coated with 80:20 $\mathrm{Au} / \mathrm{Pd}$ alloy. SEM images were captured at an accelerating voltage ranging from 5 to 20 $\mathrm{kV}$. Vega TC software were used for the microscope manipulation and capturing the images.

X-ray diffraction data were collected in the range of $3^{\circ} \leq 2 \theta \leq 30^{\circ}$ using a Philips automated powder diffractometer, Model PW 1710 with nickel-filtered $\mathrm{CuK}_{\alpha}$ radiation $(\lambda$ $=1.542 \AA$ ). MDI Datascan 3.2 software (Materials Data Inc., Livermore, CA) was used for 
data collection. The analysis was performed using MDI Jade 5.0 XRD Pattern Processing software.

\subsection{Results and Discussion}

Gelation with the biscarbamates involves the self-assembly enabled by two hydrogen bonding sites and van der Waals interactions between the alkyl side chains in solution. As discussed before, ${ }^{18}$ with an increase in the length of the alkyl chain, the relative contribution from van der Waals interactions is enhanced whereas the number of hydrogen bonds remains the same. Generally for these biscarbamate gels, as the length of the alkyl side chain increases, the minimum concentration required for gelation $\left(\mathrm{C}_{\min }\right)$ decreases (Figure 6.1) since the van der Waals interaction increases with the chain length. ${ }^{14}$ Abdallah and Weiss ${ }^{19}$ found a similar trend in the case of n-alkanes. It should be worth noting that there is an increase in $\mathrm{C}_{\min }$ when the number of carbons on the side chain is increased from 8 to 9 under microwave heating.

In our previous study on the crystallization behaviour of this series of biscarbamates, it was found that the spherulite size and growth rate are at a maximum with $\mathrm{C}_{8}$ for even biscarbamates. For the even biscarbamates, the size of the spherulites increases to $\mathrm{C}_{8}$ and then decreases significantly. The spherulite growth rate and the crystallization rate also showed a maximum with $\mathrm{C}_{8}$ and these were much smaller for longer alkyl side chains. We rationalized this behaviour on the basis of the nucleation rate as well as the rate of diffusion that are controlled by the relative contributions to the hydrogen-bond and van der Waals energy in the packing of two molecules and the two or three-dimensional growth. As the alkyl side chain length increases, the van der Waals attraction between these 
segments increases. McKierman et al. ${ }^{20}$ noted that in the case of aliphatic polyurethanes, "dilution" of the hydrogen-bond contribution relative to van der Waals interactions occurs with an increase in the length of the alkyl segment. The maximum in the rates of both the spherulitic growth as well crystallization for $\mathrm{C}_{8}$ biscarbamate prompts us to suggest that a similar dilution or compensation occurs beyond an alkyl chain length of $\mathrm{C}_{8}$. Thus, the slower rate of nucleation and growth might be a reason for the slightly higher $\mathrm{C}_{\min }$ in the case of biscarbamates. This dilution effect is more prominent in the microwave gels than the gels prepared by conventional heating since the principles of microwave chemistry does involve the bond moment of the $\mathrm{C}=\mathrm{O}$ bond in the biscarbamate.

Figure 6.1 shows that microwave heating yields gels with lower $\mathrm{C}_{\min }$ even for gelators with short alkyl chains. For the gels made using the hot plate, the minimum concentration for the biscarbamate with the short $\mathrm{C}_{6}$ side chain is $0.08 \mathrm{M}$ with benzonitrile whereas it is $0.06 \mathrm{M}$ for the gel produced using microwave heating. The reduction in the $\mathrm{C}_{\min }$ is significant with $\mathrm{C}_{7}$ to $\mathrm{C}_{10}$ side chains. Typically, with conventional heat source, biscarbamates with side chains shorter than $\mathrm{C}_{6}$ do not produce gels even at high concentrations; they would precipitate. However, with microwave heating, we were able to produce a gel with biscarbamate with the even shorter $\mathrm{C}_{5}$ side chain, at a concentration of $0.096 \mathrm{M}$. As of now, $\mathrm{C}_{5}$ is the shortest biscarbamate that can produce a gel using microwave radiation. We believe that the uniform heating on the sample due to MW radiation allows for more nucleation sites that would allow the short biscarbamate to start forming the gel fibres. Since $\mathrm{C}_{5}$ bis does not have sufficient intra-molecular forces to form these sites in conventional heating methods, it would appear that the unique heating from MW does allow for this process to occur. 


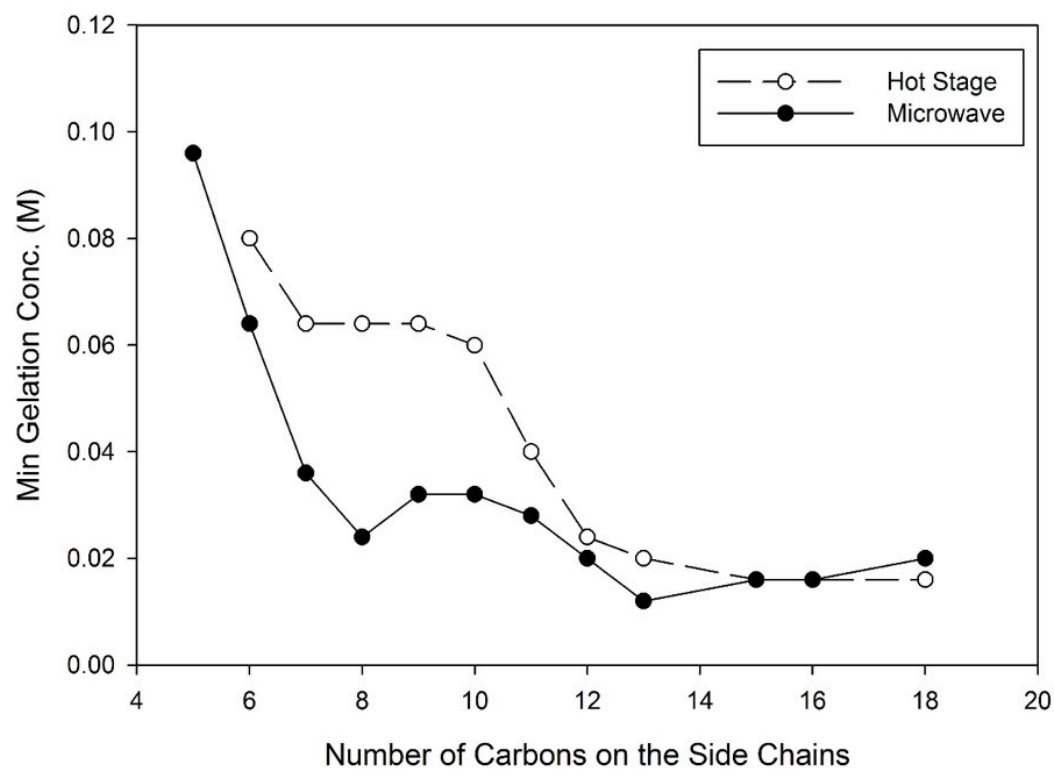

Figure 6.1. Change in the minimum gelation concentration as a function of number of carbon atoms in the alkyl side chains using the hot plate and the microwave system.

In previous reports on microwave chemistry, yields of certain organic reactions have significantly increased while the use of catalyst have remained the same or decreased..$^{21,22}$ Certain early reports described rate accelerations of several orders of magnitude over conventional heating, leading to speculation that non-thermal coupling of microwave radiation with molecules in solution can alter or reduce the activation energy of the reaction. ${ }^{12}$ This may also be similar to lowering the critical gelation energy required to produce these gels. 


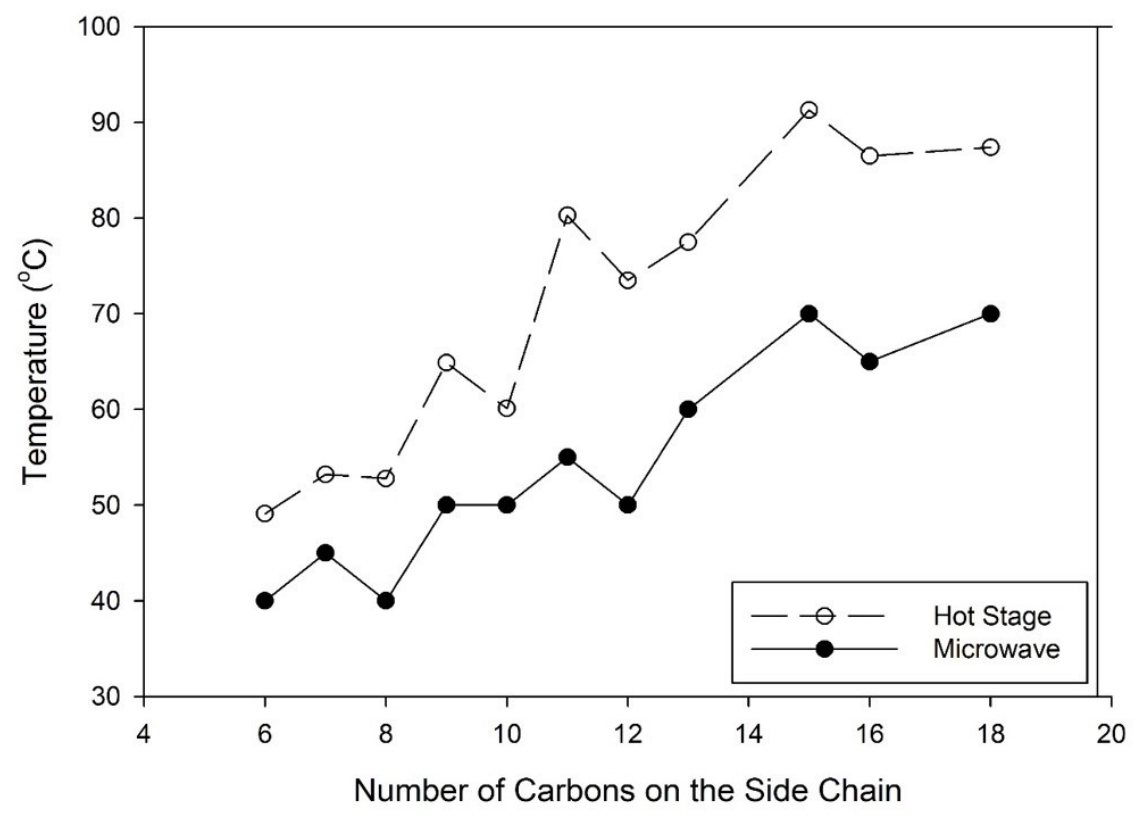

Figure 6.2. Change in the dissolution temperature of the $0.08 \mathrm{M}$ biscarbamate gel in benzonitrile made using a hotplate or a microwave system as a function of number of carbon atoms in the alkyl side chains.

Figure 6.2 shows the variation of the dissolution temperature $\left(\mathrm{T}_{\text {diss }}\right)$ with alkyl side chain length for the gels made using MW and conventional heating. This temperature relates to the temperature required to dissolve the gelator and produce the gel. We previously observed an increase in the temperature with the length of $\mathrm{C}_{n}$ side chains, where $n$ was even. ${ }^{14}$ Although the trend is similar in the present work with both even and odd numbered $\mathrm{C}_{n}$ side chains, we observe an odd-even effect, with the $\mathrm{T}_{\text {diss }}$ of an odd $\mathrm{C}_{n}$ being higher than that of succeeding even $\mathrm{C}_{n}$. We observed a similar odd-even effect in our previous study on the effect of carbon atom parity on the crystalline melting temperature 
$\left(\mathrm{T}_{\mathrm{m}}\right)$ and spherulites size of these biscarbamates. ${ }^{23}$ Those with odd $\mathrm{C}_{n}$ side chains showed a higher $T_{m}$ and larger spherulites size. We rationalized it on the basis of the syn and anticonformations of the end groups with respect to the closest ester oxygen. The inherent differences in the molecular structure would lead to different packing modes for the odd and even series. We speculate that biscarbamates with odd $\mathrm{C}_{n}$ side chains are more densely packed due to the hydrogen bonding in accordance with dispersion forces. As $\mathrm{T}_{\text {diss }}$ is related to $T_{m}$, we observe the same variation in temperature.

Figure 6.2 shows that the $\mathrm{T}_{\text {diss }}$ of the biscarbamate gels with benzonitrile produced using the MW source to be significantly lower than those of the gels prepared with the hot plate. While the difference in $\mathrm{T}_{\text {diss }}$ is about $8-12{ }^{\circ} \mathrm{C}$ with $\mathrm{C}_{6}, \mathrm{C}_{7}$ and $\mathrm{C}_{8}$ biscarbamates, it is $14-25^{\circ} \mathrm{C}$ with longer side chains. This can be attributed to the manner in which the energy gets distributed in the system. The conversion of microwave energy into thermal energy requires a medium that interacts with and absorbs microwave electromagnetic radiation in such a way that heat is generated. ${ }^{24}$ This phenomenon is dependent on the ability of a specific material (solvent or reagent) to absorb microwave energy and convert it into heat. The electric component of an electromagnetic field causes heating by two main mechanisms: dipolar polarization and ionic conduction..$^{25-27}$ Irradiation of the sample at microwave frequencies results in the molecular dipoles aligning with the applied electric field. As the dipoles rotate and align themselves energy is dissipated in the form of heat through molecular friction and dielectric loss. The amount of heat generated by this process is directly related to the ability of the matrix to align itself with the frequency of the applied field. For the homogeneous biscarbamate solutions in benzonitrile, such loss processes 
occur uniformly throughout the system, enabling the rapid and efficient volumetric heating so the solution to gel temperature is generally lower.

Apart from benzonitrile, in a previous study, ${ }^{14}$ we were able to form biscarbamate based gels with other solvents such as 1-octanol, xylene, toluene or dodecane using the conventional heating method. We attempted to prepare gels with these solvents and $\mathrm{C}_{9}$ biscarbamate using microwave heating. Figure 6.3 shows the $T_{\text {diss }}$ of the gels from different solvents for a range of gelator concentrations (starting from the minimum gelation concentration for each of the solvents), using the conventional and microwave heating methods. The dipole moments of the solvents are listed in the insets and these range from 0.07 $\mathrm{D}$ for dodecane to $4.3 \mathrm{D}$ for benzonitrile. There are some clear differences arising with the two heating methods. When conventional heating was used (Figure 6.3a), the $\mathrm{T}_{\text {diss }}$ ranged from 55 to $70{ }^{\circ} \mathrm{C}$ for the three solvents benzonitrile, 1-octanol and DMSO, with the former two exhibiting the lowest temperature. The gelation of the other three solvents required a much higher temperature range. When MW heating was used (Figure 6.3b), the $\mathrm{T}_{\text {diss }}$ recorded were found to be similar to those for the conventional heating method with the exception of dodecane where a gel was never produced and the samples yielded only precipitated solutions. However, the amount of heating time varied significantly depending on the solvents. For benzonitrile and DMSO, the microwave ramps up to the desired temperature and is heated for 30 minutes until the machine cools down which allows for the gel to set. For 1-octanol, the sample required an additional hour of MW heating to fully dissolve, and the samples in toluene and xylene required a total of two (2) hours of MW heating. These differences in the amount of heating illustrates the impact of the solvent use in the microwave. It does appear that solvents with a higher dipole moment require less 
heating to fully dissolve. The three distinct ranges correspond to (i) benzonitrile and DMSO which have a dipole moment of 4.3 and $3.96 \mathrm{D}$ respectively, showing the lowest $\mathrm{T}_{\text {diss }}$ and requiring the least amount of time (ii) 1-octanol with a smaller dipole moment of $1.76 \mathrm{D}$, requiring more heating than benzonitrile and DMSO and (iii) xylene and toluene with much smaller dipole moments requiring even more heating. Thus a clear trend is seen with the dipole moment of the solvents and the effectiveness of MW heating.

Interestingly, it is seen from Figure 6.3 that for all the solvents, the gels that were prepared using $\mathrm{MW}$ heating required a lower $\mathrm{C}_{\min }$ than those obtained from conventional heating. For example, with benzonitrile, the minimum concentration required to form gels is $0.065 \mathrm{M}$ with conventional heating, and it decreased to $0.03 \mathrm{M}$ with $\mathrm{MW}$ heating. Similar is the case with DMSO, for which $\mathrm{C}_{\min }$ decreased from $0.065 \mathrm{M}$ to $0.04 \mathrm{M}$. There is a decrease of at least $0.01 \mathrm{M}$ in $\mathrm{C}_{\min }$ for the other solvents. These results illustrate that the nature of solvent has an effect for specifically microwave heating. As for the gelator, the $\mathrm{C}_{9}$ biscarbamate has a dipole moment of $0.10 \mathrm{D}$ (as calculated from a geometrical optimization at PM3 level of theory using HyperChem and Spartan ES software), and the bond moment of the $\mathrm{C}=\mathrm{O}$ bond is $2.3 \mathrm{D}$. As discussed above, the conversion of microwave energy into thermal energy requires the solvent medium or the reagents to absorb microwave energy and convert it into heat. Solvents such as toluene and xylene with lower dipole moment do not produce enough heating for the dissolution of biscarbamate. In fact, it was observed that in increase in heating was required to dissolve these samples. 

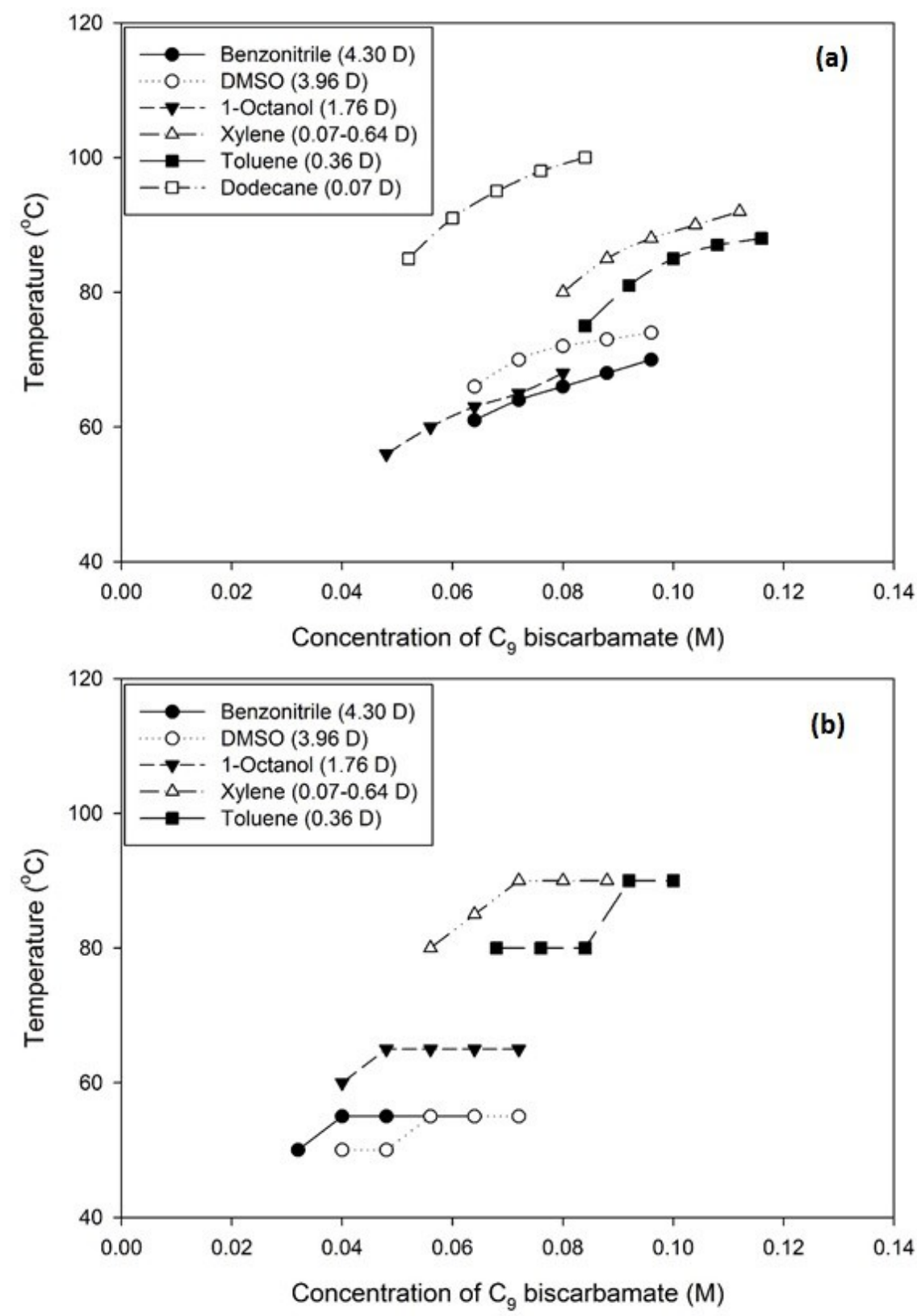

Figure 6.3. Minimum dissolution temperature for $\mathrm{C}_{9}$ biscarbamate gels in various solvents using (a) conventional and (b) microwave heating, for a range of gelator concentrations starting with the critical gelation concentration. The dipole moments of the solvents are listed in the insets. 
The fibrous morphology of the gels prepared with MW heating is similar to those made with conventional heating. Figure 6.4 shows the OM (wet gels) and SEM (xerogels), of the gels prepared by the two methods, with $\mathrm{C}_{9}$ biscarbamate and benzonitrile. There is no significant difference in the length and width of the fibres of the gels prepared by the two heating methods. Interestingly however, gels produced under microwave radiation show bundles of fibres aligned in one direction whereas this orientation is absent for gels produced using conventional heating. Such orientation of fibres was seen consistently and is not fortuitous or an artifact. We had previously prepared aligned fibres of benzonitrile/ biscarbamate gels by using a magnetic stirrer while the solution was being cooled. ${ }^{14}$ However, in the case of Figures $6.4 \mathrm{c}$ and $\mathrm{d}$ there was no magnetic stirrer involved. This might indicate that the radiation has an effect on the self-assembly of the biscarbamate molecules by reorienting and aligning the biscarbamate molecules which promotes hydrogen bonding and van der Waals interactions along a direction.

To confirm the orientation, X-ray powder diffraction traces were recorded, with the sample aligned with the intended orientation direction parallel to the direction of the incident beam and then perpendicular, by turning the sample by $90^{\circ}$ (Figure 6.5). This method was previously performed for biscarbamate xerogels where the gels were prepared with a shear force applied. ${ }^{14}$ There are two peaks of interest on the diffractogram: the intense diffraction peak at $2 \theta=25^{\circ}$ which corresponds to the distance between hydrogen bonded planes and the shorter peak at $2 \theta=13^{\circ}$. In the absence of orientation, no difference would be observed in the intensities of the diffraction peaks recorded in these two directions. However, much like the gels produced with the applied shear, the $2 \theta=25^{\circ}$ peak is slightly lower in intensity in the parallel mode than in the perpendicular mode which 
indicates that there is some preferred orientation in the sample. Additionally, the $2 \theta=13^{\circ}$ has decreased in intensity from being in the parallel to the perpendicular mode. The other non-relevant peaks indicate that, aside from the change in orientation, there is no significant structural difference.
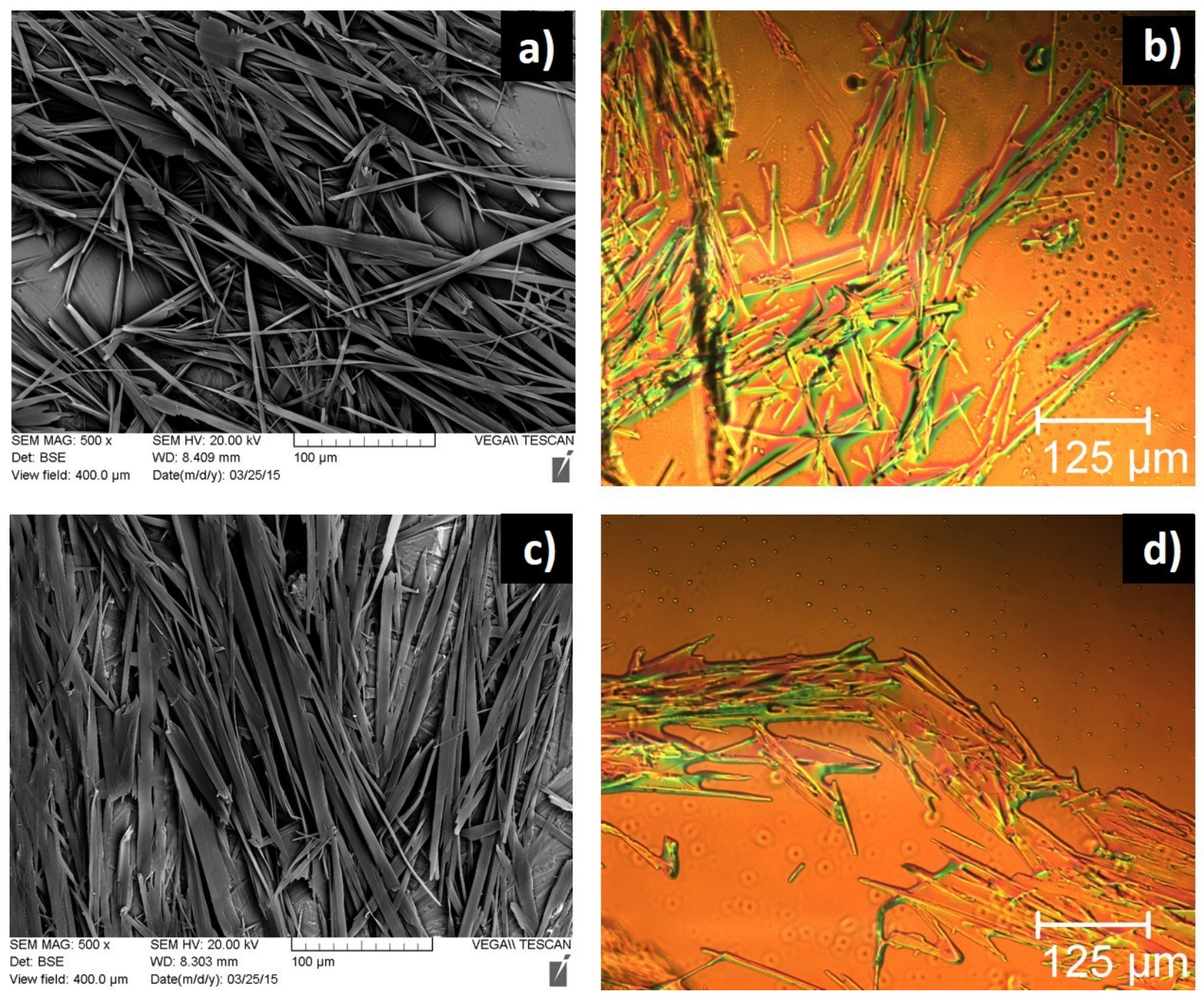

Figure 6.4. (a) SEM and (b) OM images of benzonitrile/ $\mathrm{C}_{9}$ based gels using conventional heating. (c) SEM and (d) OM images of gels made using MW source. 


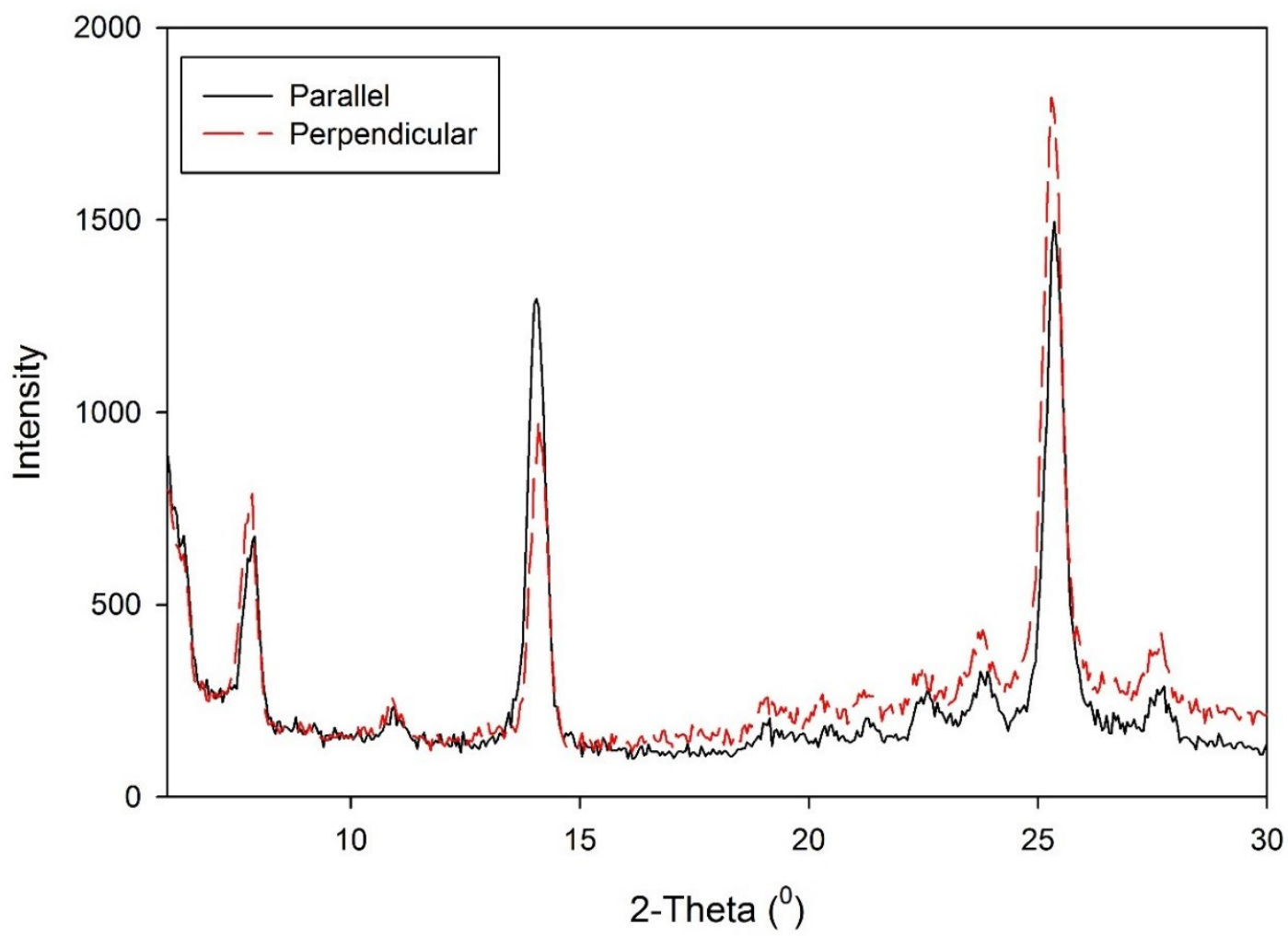

Figure 6.5. X-ray diffractogram of $\mathrm{C}_{9}$ biscarbamate dried gel. As opposed to xerogels, the dried gel was attained by drying off the excess solvent and leaving the slide exposed to air for 1 hour which would limit the appearance of an amorphous band. The designation "parallel' denotes the sample orientation along the direction of the incident X-ray beam and "perpendicular" denotes the sample rotated by $90^{\circ}$.

The morphology of the microwave produced gels, as oppose to the thermodynamic properties, is similar those produced from conventional heating. The SEM and OM images of the slow-cooled and microwave xerogels of $\mathrm{C}_{9}$ in benzonitrile are shown in Figure 6.4. Note we choose to compare the microwave produced gels with the slow-cooled 
conventional heating gels as the cooling processes are similar in time and in the cooling rate. The morphology in this case consists of shorter and thinner ribbons fibres. The $\mathrm{C}_{9}$ biscarbamate has been already reported to produce thin long fibres (462 $\mu \mathrm{m}$ long and $8 \mu \mathrm{m}$ think on average) $;{ }^{9}$ whereas the fibres produced using microwave heater are $410 \mu \mathrm{m}$ long and $3.3 \mu \mathrm{m}$ thick on average. Furthermore, the density of these fibres has greatly increased. At higher magnification, the fibres of both gels look very similar. These occurrences are also observed for even biscarbamates. Figure 6.6 show SEM images of $\mathrm{C}_{12}$ xerogels obtained from conventional and microwave heating. The slow-cooled $\mathrm{C}_{12}$ biscarbamate xerogels also show thin long and thin fibres using $(350 \mu \mathrm{m}$ long and $5.3 \mu \mathrm{m}$ thick on average). The microwave gels show the fibres $250 \mu \mathrm{m}$ long and $3 \mu \mathrm{m}$ thick on average.

The SEM images of the slow-cooled and microwave xerogels of $\mathrm{C}_{9}$ in DMSO, 1octanol, xylene and toluene are shown in Figure 6.7. Since organogels in dodecane were never produced using microwave, they are not included. In all the microwave xerogels, the samples show a denser surface with smaller structures. In addition, there seem to be a direction either along the growth sites or there would be some alignment along the structures themselves. These observations seem to indicate that the microwave heating method affects the number of growth sites and the direction of the growth. Since the structures appear to be denser for the microwave gel, it suggests that the number of growth sites have dramatically increased. This may also explain why gels produced by microwave heating require a lower concentration. 


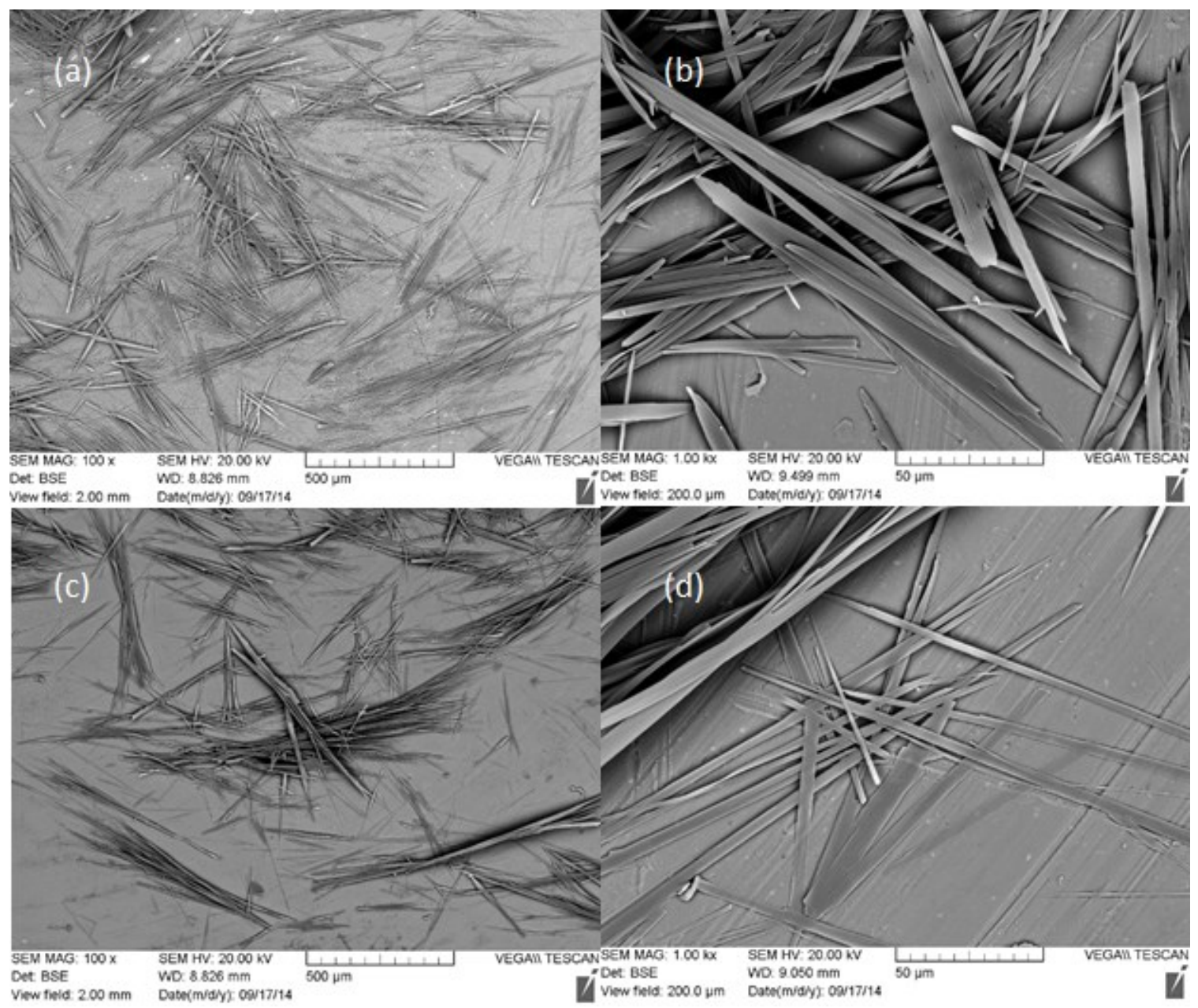

Figure 6.6. (a) Low and (b) high magnification SEM images of $\mathrm{C}_{12}$ biscarbamate xerogels from benzonitrile made using a hot plate and (c) low and (d) high magnification SEM images of xerogels made using a microwave digestive system. 

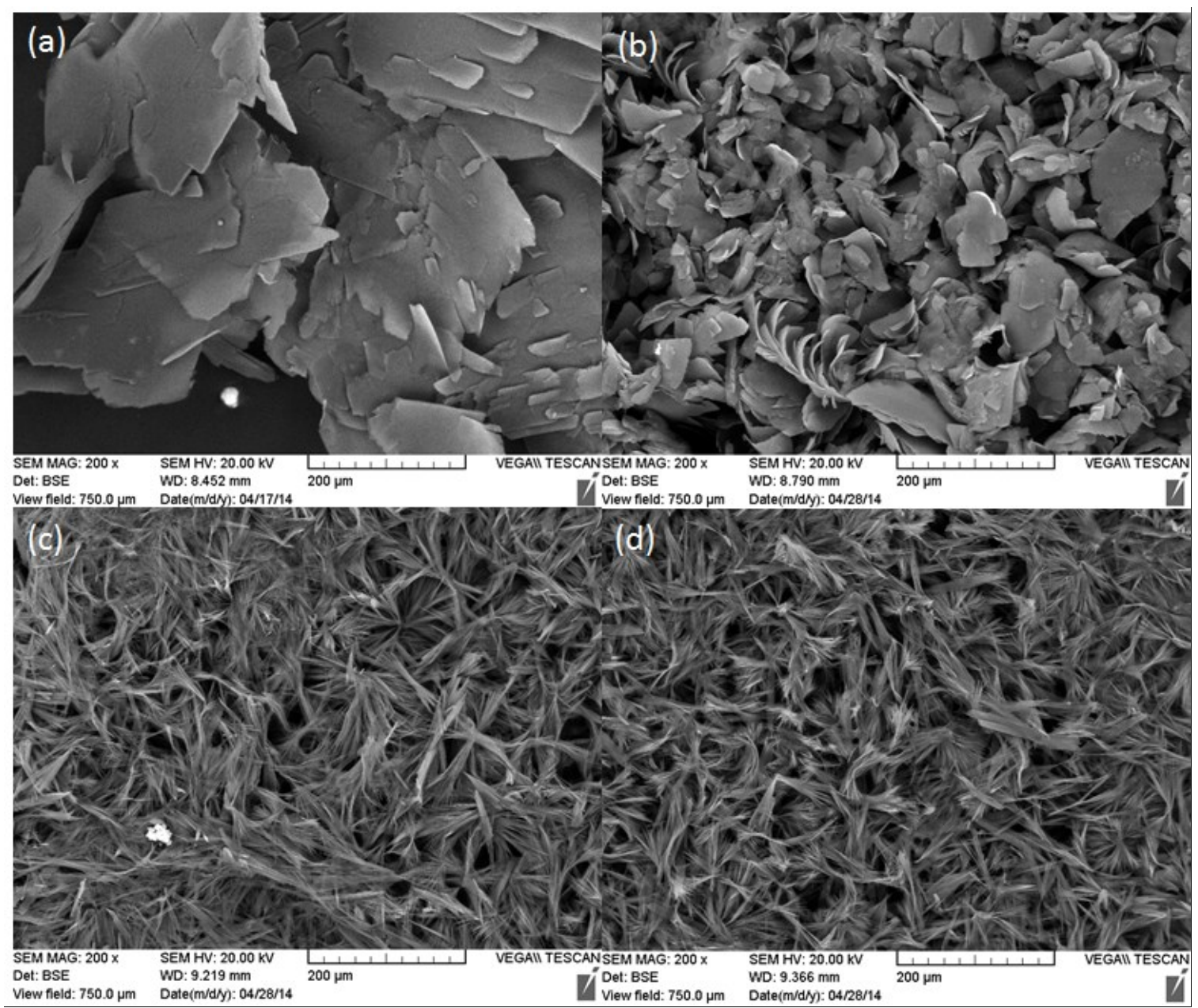

(Figure 6.7 cont'd on next page) 


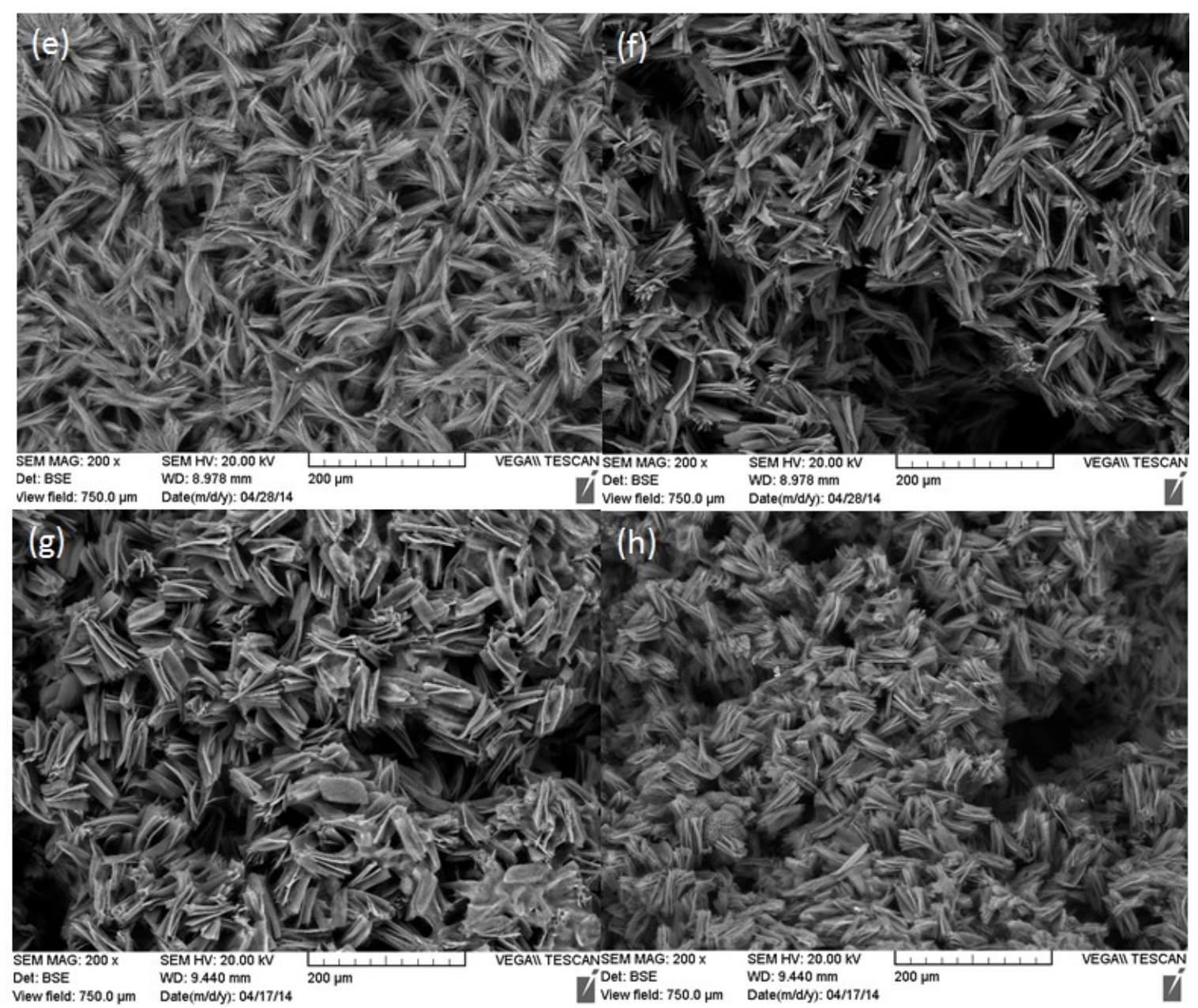

Figure 6.7. $\mathrm{SEM}$ images of $\mathrm{C}_{9}$ biscarbamate xerogels from $(\mathrm{a}, \mathrm{b}) \mathrm{DMSO},(\mathrm{c}, \mathrm{d})$ 1-octanol, $(\mathrm{e}, \mathrm{f})$ xylene, and $(\mathrm{g}, \mathrm{h})$ toluene made using (left) a hot plate and (right) a microwave digestive system.

We compare the gel-to-solution temperatures ( $\left.T_{\text {gel-sol }}\right)$ for the gels obtained using conventional and microwave heating methods (Table 6.1). The differences in $\mathrm{T}_{\text {gel-sol }}$ vary depending on the solvent used; this is dependent on the gel strength in the solvents. Interestingly, despite the differences in dissolution temperatures for the production of the gels, $\mathrm{T}_{\text {gel-sol }}$ are very similar in value. This indicates that both heating methods produce gels 
with similar physical properties. The fact that the morphology of these gels in benzonitrile and DMSO also show similarities further illustrates that both heating methods yield comparable gels. The main differences between both techniques are the critical gelation concentration and the temperature required to dissolve the initial components. From these finding, we were also able to assess the $\mathrm{T}_{\text {gel }}$ for the microwave gels as we were unable to determine them experimentally. Considering how alike $T_{\text {gel-sol }}$ are for both methods, we can infer that the $T_{\text {gel }}$ will be in the same range as for the gels produced using conventional heating.

Table 6.1. The gel-to-solution temperatures for gels produced using both conventional heating and microwave heating methods, and the gelation temperature for the conventional heated gels. Due to limitation of the microwave apparatus, the gelation temperature cannot be determined for this method.

\section{Conventional Heating Microwave}

\begin{tabular}{cccc}
\hline Solvents & $\mathrm{T}_{\text {gel-sol }}\left({ }^{\circ} \mathrm{C}\right)$ & $\mathrm{T}_{\text {gel }}\left({ }^{\circ} \mathrm{C}\right)$ & $\mathrm{T}_{\text {gel-sol }}\left({ }^{\circ} \mathrm{C}\right)$ \\
\hline Benzonitrile & 64.6 & 59.0 & 65.4 \\
DMSO & 82.2 & 63.1 & 81.8 \\
1-Octanol & 59.7 & 33.5 & 56.2 \\
Xylene & 69.3 & 58.8 & 69.6 \\
Toluene & 65.5 & 56.5 & 66.9 \\
Dodecanol & 86.3 & 59.4 & N.A. \\
\hline
\end{tabular}




\subsection{Conclusions}

In conclusion, we have shown that microwave radiation could be used to prepare organogels of biscarbamates. With benzonitrile as the solvent, for biscarbamates with various alkyl side chain lengths, both the minimum concentration $\left(\mathrm{C}_{\text {min }}\right)$ required for gelation and the temperature required to produce the gel decreased with MW heating compared to the conventional. Gels prepared with different solvents show that the dipole moment of the solvent plays a role in these properties when MW is used. Solvents such as benzonitrile and DMSO are more effective in this regard than xylene or toluene. In addition, it was possible to prepare gels with the biscarbamate with a short alkyl side chain using MW, while it was not with conventional heating. Irrespective of the different opinions in the literature on the effectiveness of MW radiation in chemical processes, the differences that we observed between the conventional and MW heating methods do point to the merit of the latter. Although it may be said that a hot plate is cheaper to buy than the MW system, from the perspective of scientific novelty we have demonstrated that the latter has the advantage of reducing the gel forming temperature and the concentration required. 


\subsection{References}

(1) Vázquez, E.; Giacalone, F.; Prato, M. Non-Conventional Methods and Media for the Activation and Manipulation of Carbon Nanoforms. Chem. Soc. Rev. 2014, 43 (1), $58-69$.

(2) Giguere, R. J.; Bray, T. L.; Duncan, S. M.; Majetich, G. Application of Commercial Microwave Ovens to Organic Synthesis. Tetrahedron Lett. 1986, 27 (41), 49454948.

(3) Gedye, R.; Smith, F.; Westaway, K.; Ali, H.; Baldisera, L.; Laberge, L.; Rousell, J. The Use of Microwave Ovens for Rapid Organic Synthesis. Tetrahedron Lett. 1986, 27 (3), 279-282.

(4) Larhed, M.; Hallberg, A. Microwave-Promoted Palladium-Catalyzed Coupling Reactions. J. Org. Chem. 1996, 61 (26), 9582-9584.

(5) Polshettiwar, V.; Varma, R. S. Microwave-Assisted Organic Synthesis and Transformations Using Benign Reaction Media. Acc. Chem. Res. 2008, 41 (5), 629639.

(6) Caddick, S. Microwave Assisted Organic Reactions. Tetrahedron 1995, 51 (38), $10403-10432$.

(7) Durand-Reville, T.; Gobbi, L. B.; Gray, B. L.; Ley, S. V; Scott, J. S. Highly Selective Entry to the Azadirachtin Skeleton via a Claisen Rearrangement/Radical Cyclization Sequence. Org. Lett. 2002, 4 (22), 3847-3850.

(8) Loupy, A.; Petit, A.; Hamelin, J.; Texier-Boullet, F.; Jacquault, P.; Mathe, D. New 
Solvent-Free Organic Synthesis Using Focused Microwaves. Synthesis (Stuttg). 1998, No. 9, 1213-1234.

(9) Varma, R. Solvent-Free Organic Syntheses. Using Supported Reagents and Microwave Irradiation. Green Chem. 1999, 1 (1), 43-55.

(10) Selvakumar, P.; Sarojadevi, M.; Sundararajan, P. Synthesis, Characterization and Microwave-Enhanced Polymerization of a Phthalonitrile Resin. Mater. Sci. Eng. B 2010, 168 (1), 214-218.

(11) Nüchter, M.; Ondruschka, B.; Jungnickel, A.; Müller, U. Organic Processes Initiated by Non-Classical Energy Sources. J. Phys. Org. Chem. 2000, 13 (10), 579-586.

(12) Rosana, M. R.; Hunt, J.; Ferrari, A.; Southworth, T. A.; Tao, Y.; Stiegman, A. E.; Dudley, G. B. Microwave-Specific Acceleration of a Friedel-Crafts Reaction: Evidence for Selective Heating in Homogeneous Solution. J. Org. Chem. 2014, 79 (16), 7437-7450.

(13) Chen, P.-K.; Rosana, M. R.; Dudley, G. B.; Stiegman, A. E. Parameters Affecting the Microwave-Specific Acceleration of a Chemical Reaction. J. Org. Chem. 2014, $79(16), 7425-7436$.

(14) Khanna, S.; Khan, M. K.; Sundararajan, P. Influence of Double Hydrogen Bonds and Alkyl Chains on the Gelation of Nonchiral Polyurethane Model Compounds: Sheets, Eaves Trough, Tubes and Oriented Fibers. Langmuir 2009, 25 (22), 1318313193.

(15) Khan, M. K.; Sundararajan, P. Encapsulation of Dye Molecules and Nanoparticles 
in Hollow Organogel Fibers of a Nonchiral Polyurethane Model Compound. Chem. Eur. J. 2011, 17 (4), 1184-1192.

(16) Moniruzzaman, M.; Sundararajan, P. R. Low Molecular Weight Organogels Based on Long-Chain Carbamates. Langmuir 2005, 21 (9), 3802-3807.

(17) Goodbrand, B.; Boils, D. C.; Sundararajan, P. R.; Wong, R. W. US Patent 6,414,051, 2002.

(18) Khanna, S.; Moniruzzaman, M.; Sundararajan, P. R. Influence of Single versus Double Hydrogen-Bonding Motif on the Crystallization and Morphology of SelfAssembling Carbamates with Alkyl Side Chains: Model System for Polyurethanes. J. Phys. Chem. B 2006, 110 (31), 15251-15260.

(19) Abdallah, D. J.; Weiss, R. G. N-Alkanes Gel N-Alkanes (and Many Other Organic Liquids). Langmuir 2000, 16 (2), 352-355.

(20) McKiernan, R. L.; Heintz, A. M.; Hsu, S. L.; Atkins, E. D. T.; Penelle, J.; Gido, S. P. Influence of Hydrogen Bonding on the Crystallization Behavior of Semicrystalline Polyurethanes. Macromolecules 2002, 35 (18), 6970-6974.

(21) Ranu, B. C.; Hajra, A.; Jana, U. Microwave-Assisted Simple Synthesis of Quinolines from Anilines and Alkyl Vinyl Ketones on the Surface of Silica Gel in the Presence of Indium (III) Chloride. Tetrahedron Lett. 2000, 41 (4), 531-533.

(22) Mehta, V. P.; Van der Eycken, E. V. Microwave-Assisted C-C Bond Forming Cross-Coupling Reactions: An Overview. Chem. Soc. Rev. 2011, 40 (10), 4925- 
4936.

(23) Khan, M. K.; Sundararajan, P. R. Effects of Carbon Atom Parity and Alkyl Side Chain Length on the Crystallization and Morphology of Biscarbamates, A Set of Model Compounds for Polyurethanes. J. Phys. Chem. B 2011, 115 (27), 8696-8706.

(24) Metaxas, A. C. and; Meredith, R. J. Industrial Microwave Heating; IET, 1983.

(25) Stass, D. V; Woodward, J. R.; Timmel, C. R.; Hore, P. J.; McLauchlan, K. A. Radiofrequency Magnetic Field Effects on Chemical Reaction Yields. Chem. Phys. Lett. 2000, $329(1-2), 15-22$.

(26) Timmel, C. R.; Hore, P. J. Oscillating Magnetic Field Effects on the Yields of Radical Pair Reactions. Chem. Phys. Lett. 1996, 257 (3-4), 401-408.

(27) Thostenson, E. T.; Chou, T.-W. Microwave Processing: Fundamentals and Applications. Compos. Part A Appl. Sci. Manuf. 1999, 30 (9), 1055-1071. 


\section{CHAPTER 7}

Conclusions and Recommendations for Future Works 


\section{CHAPTER VII: CONCLUSIONS AND RECOMMENDATIONS FOR FUTURE WORKS}

\subsection{Conclusions}

The purpose of this thesis was to examine the effects of blending and gelation of a series of hydrogen bond-mediated self-assembling biscarbamates. Their crystallization and gelation behaviours, their physical properties, and their morphology were evaluated throughout this project.

In the introduction section (Chapter 1), the effects of different noncovalent interactions (i.e. hydrogen bonding, van der Waals interactions, $\pi$ - $\pi$-stacking, etc.) on the morphology of the self-assembling molecules have been discussed. In certain cases more than one of these factors have been found to be responsible for the self-assembly of the molecules. We focused on the self-assembly of biscarbamates, a model compound of polyurethanes with two hydrogen bond motifs separated by an alkyl segment and having terminal alkyl chains of lengths ranging from $\mathrm{C}_{3}$ to $\mathrm{C}_{18}$.

We first looked at the miscibility of the biscarbamates by blending two molecules with similar spacer but different alkyl side chain lengths. It was found that these molecules are capable of hydrogen-bond mediated self-assembly leading to specific association between the same types but do not involve mutual intercalation at the molecular level. This implies that they are immiscible during blending and that they self-sort. While self-sorting might be advantageous for separation of blends, it poses a challenge for modifying properties such as the melting temperatures, spherulite size, etc for various applications. It was believed that the key to miscibility is the crystallization rate of each molecule. In an 
attempt to control the rate of crystallization, solvent-based blends of two biscarbamate with varying side chain lengths were made. It was shown that there is a smaller change in the melting temperatures caused by the diluent effect for the solvent-based blends compared to the melt-based blends. In addition, the crystallization of the blends has changed significantly as seen by the FWHM of the thermographs from the DSC and the XRD patterns. This was an indication of the presence of the solvent limiting the self-sorting behaviour by controlling the rate of crystallization. The closer the crystallization rate, the more miscible the blend becomes. It turns out that biscarbamates with similar crystallization rates usually have similar side chains lengths and thus similar melting temperatures. However, we did indeed show that is was possible to prepare miscible blends of two molecules with different melting temperatures by using a compatibilizer. The components of the blend exercise a mutual diluent effect, thereby reducing the melting temperatures, spherulite size, crystallinity, and the heat of fusion. Isothermal crystallization studies showed that the growth rates of the spherulites are also reduced upon blending, compared to those of the individual components. In situ observations during the melting of the blends revealed that the spherulites of each constituent do not form separately, but by mixing with and providing nucleating sites for each other. The extent of hydrogen bonding and the packing of alkyl side chains were not affected by blending. The variation in the length of the alkyl side chains plays a role in the immiscibility and selectivity of these molecules during crystallization that parallels those of monocarbamates and hydrogen bond-mediated self-assembling polymers.

Additionally, we examined the behaviour and morphology of organogels made from the series of biscarbamate molecules. Biscarbamate molecules with a $\left(\mathrm{CH}_{2}\right)_{6}$ spacer 
between the hydrogen bonding motifs with an odd or even number of carbon atoms in the alkyl side chains showed an odd-even alternation in gel-to-solution temperatures analogous to the deviations of the melting temperatures observed for the biscarbamate crystals. We justify this occurrence by the influence of the packing order of the carbon atom parity in alkyl side chains leading to a different thermal behaviour. It is seen that the fibre size increases with the increase in alkyl side chain length reaching a maximum at $\mathrm{C}_{13}$ and then decreases. In addition, it seems like the length of the ribbons is altered slightly by the preparation method (i.e., quenching versus slow-cooling). Hence the length and carbon atom parity of the alkyl side chains as well as the sample preparation protocol play an important role in the morphology of these molecules. These methods did not affect the aging, the thermo-properties or the stability of these gels. We also looked at the growth of the ribbons at various time lengths. It appears that the fibres continue to grow after the formation, but the growth dramatically decreases after a week. Hence, we conclude that the thermal properties and the morphology of these molecules can be tailored to the desired functional level by changing the alkyl side chain length with the right carbon atom parity.

We then looked at biscarbamate gel blends where we observed different blending behaviours: sergeant-soldier, majority rules and self-sorting. For any blends with biscarbamates containing less than six carbon groups along the side chain and that cannot gel on their own, the fibres resembles the longer biscarbamate component. This may be due to the fact that the shorter biscarbamate cannot grow on their own; hence, the resulting gels appear to have a sergeant-soldier type behaviour. Also, it appears that the $\mathrm{C}_{8} / \mathrm{C}_{9}$ gels also yield fibres that resemble the longer biscarbamate $\left(\mathrm{C}_{9}\right)$. As the concentration of the longer biscarbamate decreases, the resulting gel fibres continue to resemble the $\mathrm{C}_{9}$ only 
gels. These are the only two cases of sergeant-soldier effect observed. Gel formation for a slow-cooled sample of $0.08 \mathrm{M} \mathrm{C}_{8}$ and $\mathrm{C}_{9}$ biscarbamate require 28.5 and 26.5 minutes respectively for them to fully set. The trend of the $T_{\text {gel-sol }}$ for various concentration shows the two biscarbamates would lead to a miscible blend. However, since the time of gelation for $\mathrm{C}_{9}$ biscarbamate is slightly lower, we hypothesize that the $\mathrm{C}_{9}$ molecules would drive the assembly for the rest of the blend resulting in the sergeant-soldier behaviour. For any odd-odd gels blends with a larger difference in side chain lengths, such as $\mathrm{C}_{7} / \mathrm{C}_{11}$, we observed a majority rules effect. The fibre morphology is determined by the component with the higher concentration. In addition, there appears to have two endotherms which are reproducible throughout many cycles. Finally, for certain odd-odd biscarbamate mixtures where the difference in the size chain is short, we observed a self-sorting effect. Both types of fibres are shown in the scanning electron micrographs of the xerogel. The DSC also showed a single endotherm in the first cycle, but the following cycles showed multiple endotherms. A hypothesis for the change in thermal behaviour is that the allotted time to rest after the setting of the gel may have allowed the molecules to rearrange and form a more thermal miscible blend. During the heating and cooling cycles in the DSC, the gel did not have a sufficient amount of time to allow for such rearrangements. The formation of the individual components were observed visually.

Lastly, we have shown that microwave radiation could be used to prepare organogels of biscarbamates. Biscarbamates with alkyl side chain lengths varying from $\mathrm{C}_{5}$ to $\mathrm{C}_{18}$ were used, with five solvents (benzonitrile, DMSO, 1-octanol, xylene and toluene). A gel with dodecane was never produced under microwave radiation as opposed to conventional heating. The minimum gelation concentration and the amount of heating for 
dissolution were significantly reduced with MW heating for all the side chain lengths of the biscarbamates. In addition, it was possible to prepare gels with a biscarbamate with short alkyl side chain using MW, while it was not possible with conventional heating. While such reduction was pronounced with benzonitrile and dimethyl sulfoxide with large dipole moments, the amount of heating for dissolution increased with MW heating (compared to the conventional) in the case of xylene and toluene with small dipole moments. This has to do with the unique manner in which microwave radiation reorient and realign the molecule while perpetuating a contained heating system. An inherent orientation of these fibers was seen with MW heating.

\subsection{Recommendations for Future Work}

In our past research, we investigated the application of biscarbamate gels in the development of a polymer matrix. Such porous materials involving our biscarbamates present a novel and worthwhile method to generate porous resins at lower costs. We studied the heterologous blends of biscarbamates with biocompatible polycaprolactone (PCL) as an example. ${ }^{1}$ When they were dissolved together in chloroform, within a narrow range of alkyl side chain lengths, they formed a gel upon cooling the solution. Biscarbamates formed the hollow gel fibers which were embedded in the PCL matrix and immobilized the solvent. The hollow nature of the gel fibers resulted in microchannels and macrovoids, depending on the alkyl side chain length of the biscarbamate. It was found that the extent of porosity and of the pore dimensions could be tailored by the right choice of biscarbamate molecules. Impregnation of the gel fibers in the polymer matrix enhanced the mechanical 
robustness of the gels. Thus, it was concluded that we were able to fabricate twocomponent physical gels comprised of an immiscible pair of a low molecular weight organogelator and a biocompatible polymer. With these impressive properties in mind, we intend to look further into the development of biscarbamate gels.

\subsubsection{Cyano-Biscarbamates}

There have been similar studies on cross-linking monomer gels to yield porous gelation network. Typically, a physical gel of the monomer molecules is subjected to an energy source (either thermal, pressure, change in $\mathrm{pH}$, or radiation), and the monomers would polymerize via cross-linking. In a collaborate work, ${ }^{2}$ organogels were prepared from dicyanate ester monomers with a polar aprotic solvent. When single wall carbon nanotubes (CNT) were added into the monomer solution prior to gelation, the resulting mixture led to nano-composite gels where the polymerization temperature and the curing time of the cyanate esters decreased with an increase in CNT loading. The morphology of the polycyanurate network consisted of porous fibrous network. Indeed, the concept of forming nanocomposite with nitrile and CNTs is still evolving. ${ }^{3-6}$ The cross-linking process involving our biscarbamates presents a novel and worthwhile method to generate porous resins at lower costs.

In a similar manner, we intend to study cross-linkable biscarbamate molecules in the goal of polymerizing them afterwards. A nitrile-terminated version of the biscarbamate can be synthesized when the aliphatic alcohol is replaced by a hydroxynitrile. This would lead to two nitrile groups at the ends of the biscarbamates. It would be very interesting to study their morphology, their crystallization behaviour, and their gel behaviour as well as 
their cross-linking potential and their stability. Many other developments can arise from the cyano-biscarbamates such as films, coatings, and foams.

\subsubsection{Aerogels}

Another approach into the development of highly porous materials is to develop aerogels using our biscarbamate molecules. Aerogels are quasi-stable, low-density, threedimensional assemblies of nanoparticles and typically are derived from drying wet-gels by turning the pore-filling solvent into a supercritical fluid (SCF). As a result, the dry samples keep the very unusual porous texture which they had in the wet stage. In general these dry solids have very low apparent densities, large specific surface areas, and in most cases exhibit amorphous structures. In addition, they are metastable from the point of view of their thermodynamic properties. The combination of those properties is extremely attractive for many practical applications. The resulting wet-gels would be dried to aerogels with $\mathrm{SCF} \mathrm{CO}_{2}$. The motivation of that work was the synthesis of high impact strength aerogels, with similar biscarbamate properties. 


\subsection{References}

(1) Khan, M. K.; Chan, A.; Sundararajan, P. R. Two Component Gels of Immiscible Blends of Biscarbamates (polyurethane Model Compounds) and Poly ( $\varepsilon$ Caprolactone). Soft Matter 2012, 8 (39), 10149-10157.

(2) Rakesh, S.; Sakthidharan, C. P.; Sarojadevi, M.; Sundararajan, P. R. Monomer Self Assembly and Organogelation as a Route to Fabricate Cyanate Ester Resins and Their Nanocomposites with Carbon Nanotubes. Eur. Polym. J. 2015, 68, 161-174.

(3) Zhan, Y.; Yang, X.; Guo, H.; Yang, J.; Meng, F.; Liu, X. Cross-Linkable Nitrile Functionalized Graphene Oxide/poly (arylene Ether Nitrile) Nanocomposite Films with High Mechanical Strength and Thermal Stability. J. Mater. Chem. 2012, 22 (12), 5602-5608.

(4) Lee, S. Y.; Kang, N. R.; Shin, D. W.; Lee, C. H.; Lee, K.-S.; Guiver, M. D.; Li, N.; Lee, Y. M. Morphological Transformation during Cross-Linking of a Highly Sulfonated Poly (phenylene Sulfide Nitrile) Random Copolymer. Energy Environ. Sci. 2012, 5 (12), 9795-9802.

(5) Likozar, B.; Major, Z. Morphology, Mechanical, Cross-Linking, Thermal, and Tribological Properties of Nitrile and Hydrogenated Nitrile Rubber/multi-Walled Carbon Nanotubes Composites Prepared by Melt Compounding: The Effect of Acrylonitrile Content and Hydrogenation. Appl. Surf. Sci. 2010, 257 (2), 565-573.

(6) Peters, K.; Richards, F. M. Chemical Cross-Linking: Reagents and Problems in Studies of Membrane Structure. Annu. Rev. Biochem. 1977, 46 (1), 523-551. 
APPENDIX A

FTIR Spectra of the Biscarbamates 


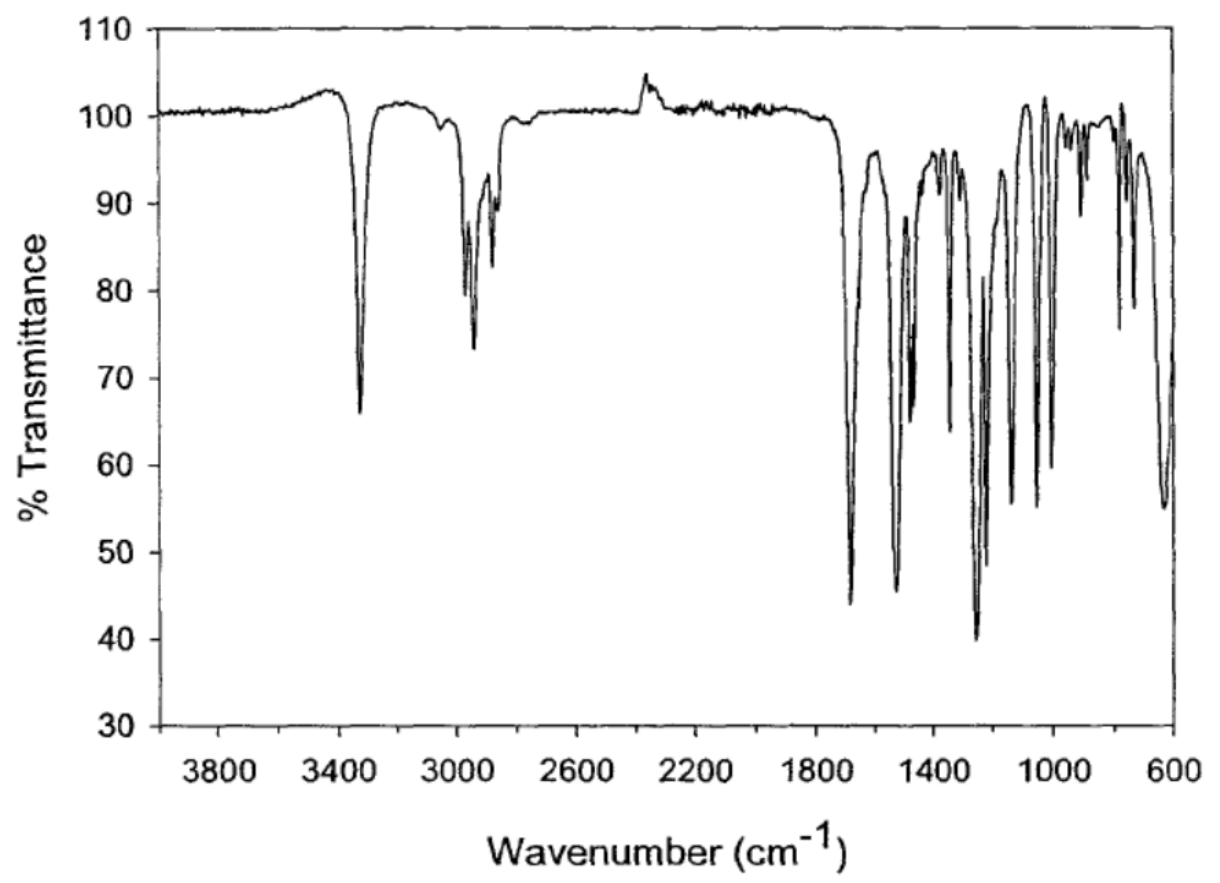

Figure A.1. FTIR spectrum of $\mathrm{C}_{3}$ biscarbamate.

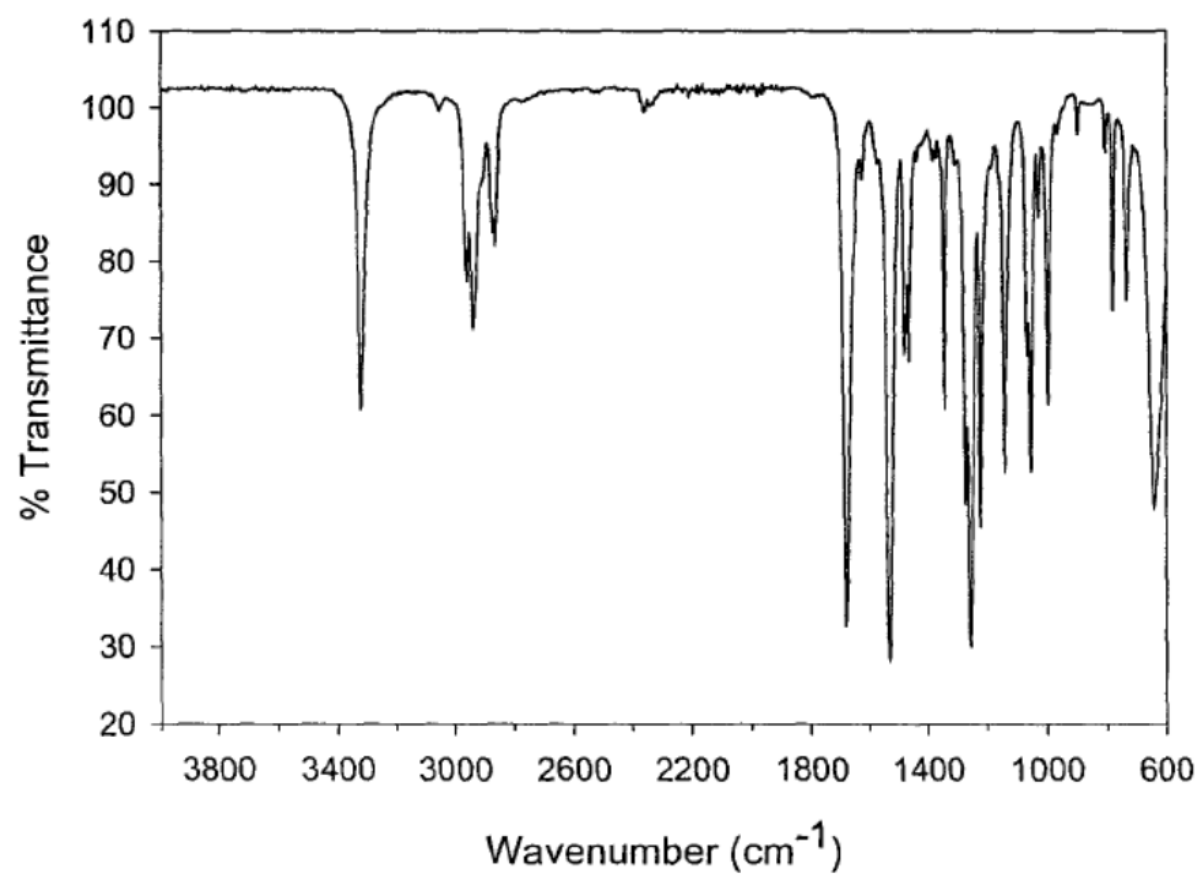

Figure A.2. FTIR spectrum of $\mathrm{C}_{4}$ biscarbamate. 


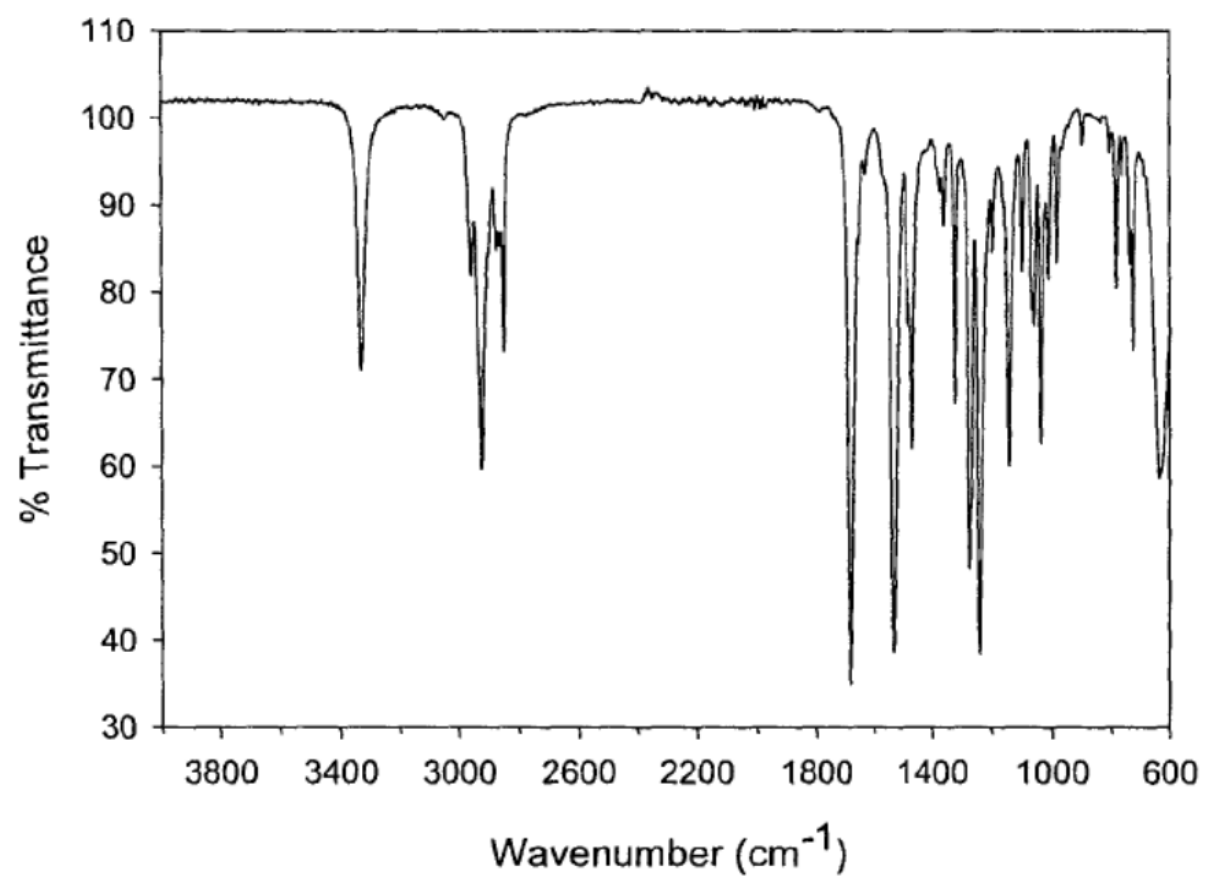

Figure A.3. FTIR spectrum of $\mathrm{C}_{5}$ biscarbamate.

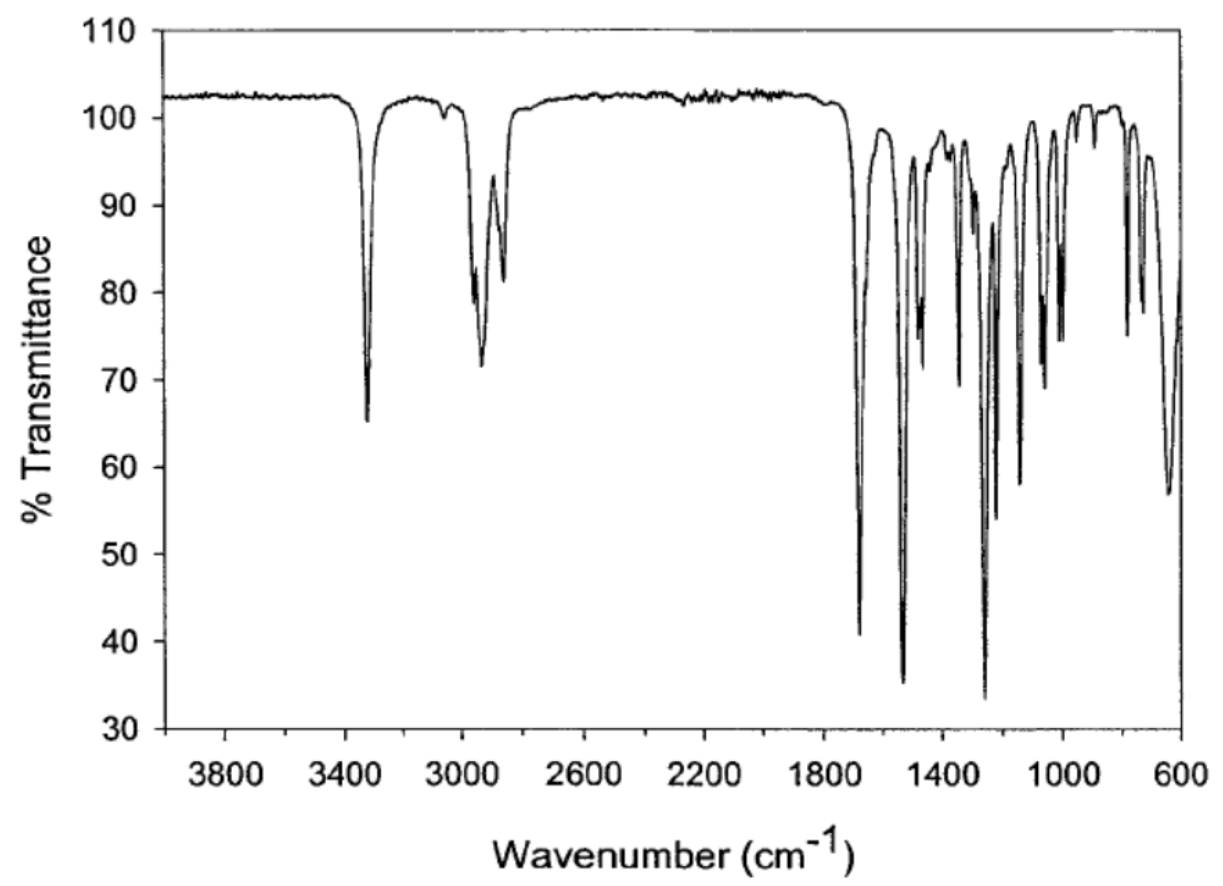

Figure A.4. FTIR spectrum of $\mathrm{C}_{6}$ biscarbamate. 


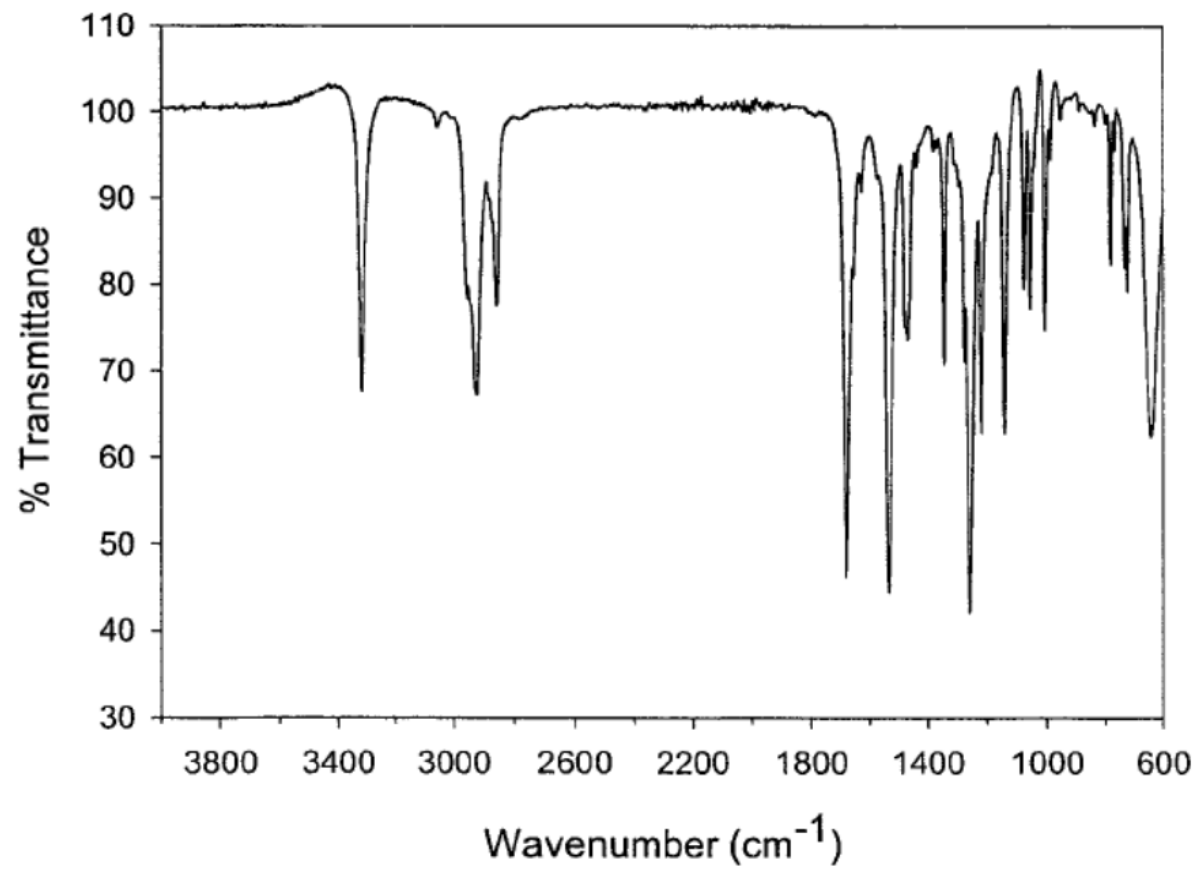

Figure A.5. FTIR spectrum of $\mathrm{C}_{7}$ biscarbamate.

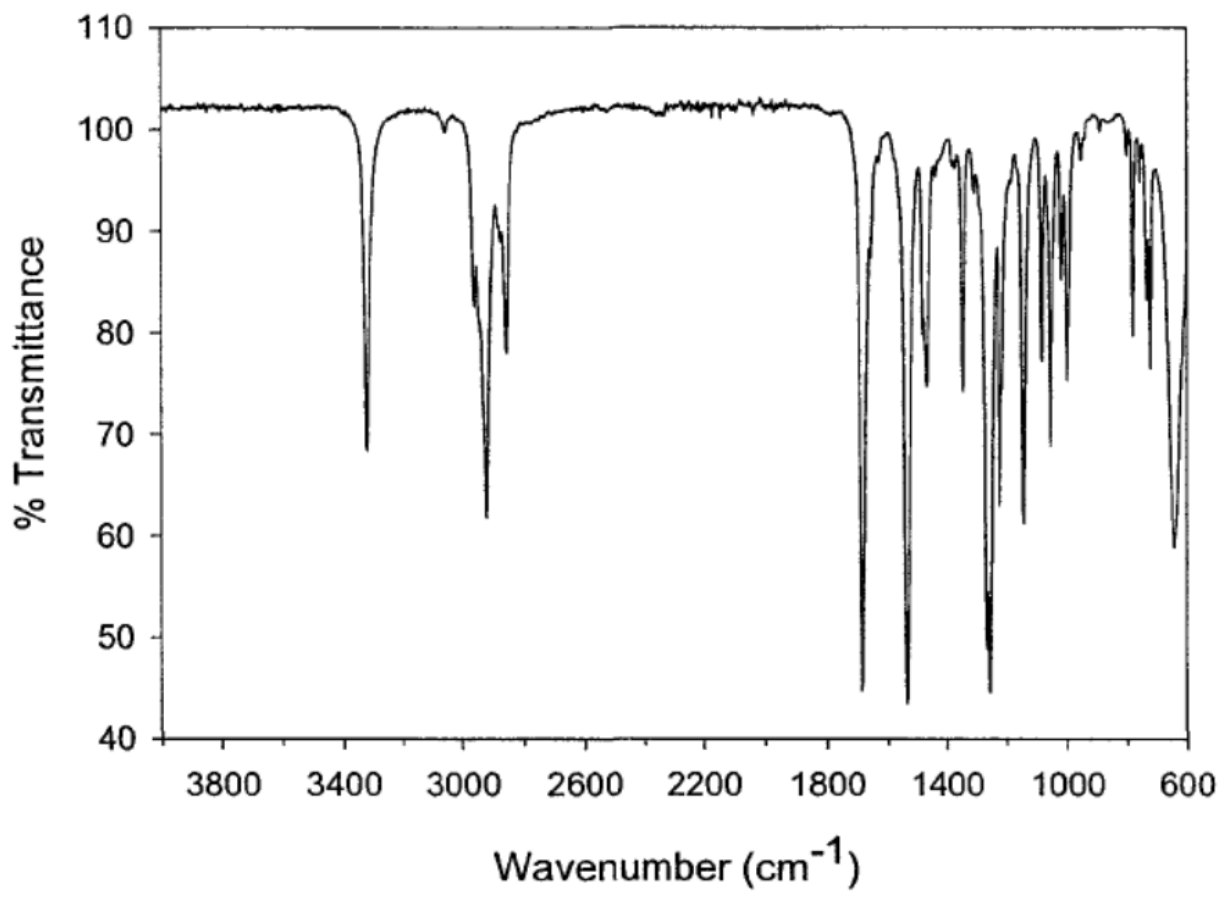

Figure A.6. FTIR spectrum of $\mathrm{C}_{8}$ biscarbamate. 


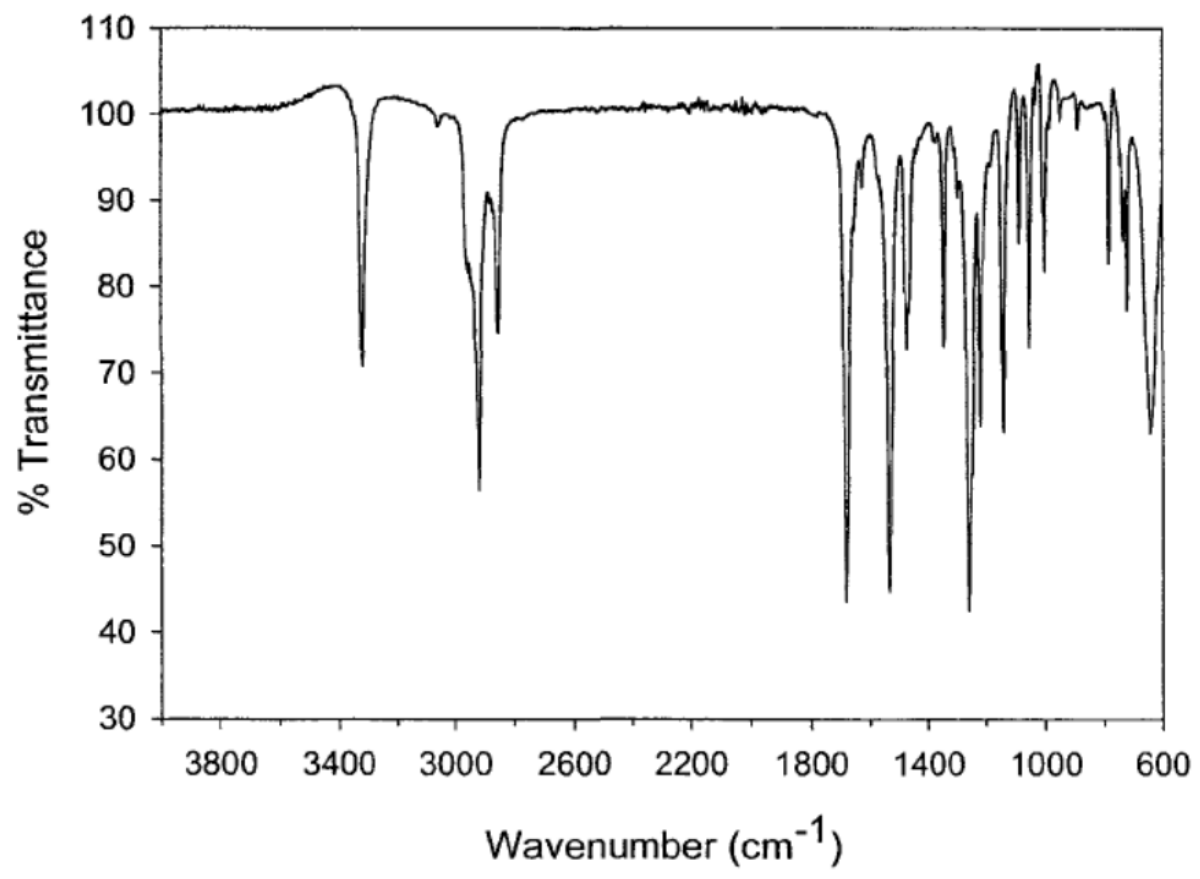

Figure A.7. FTIR spectrum of $\mathrm{C}_{9}$ biscarbamate.

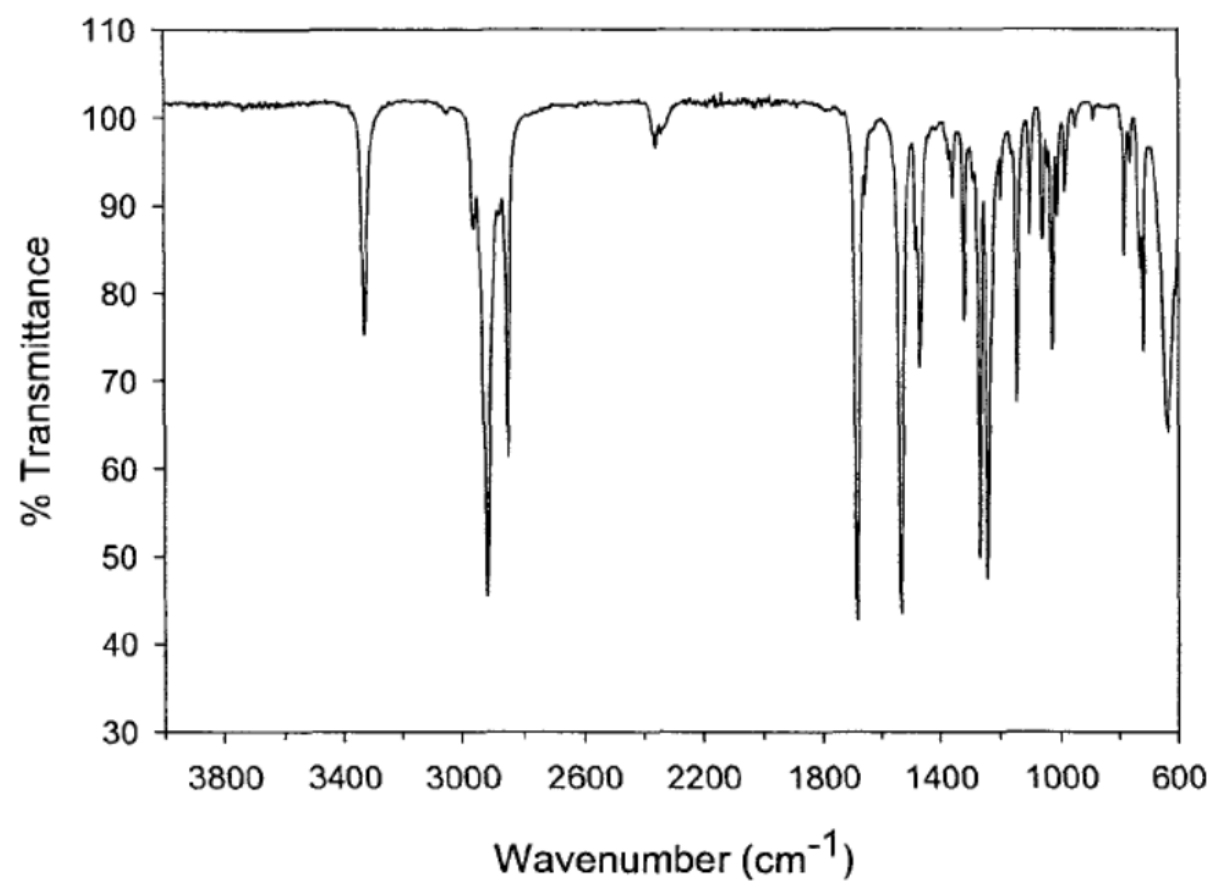

Figure A.8. FTIR spectrum of $\mathrm{C}_{10}$ biscarbamate. 


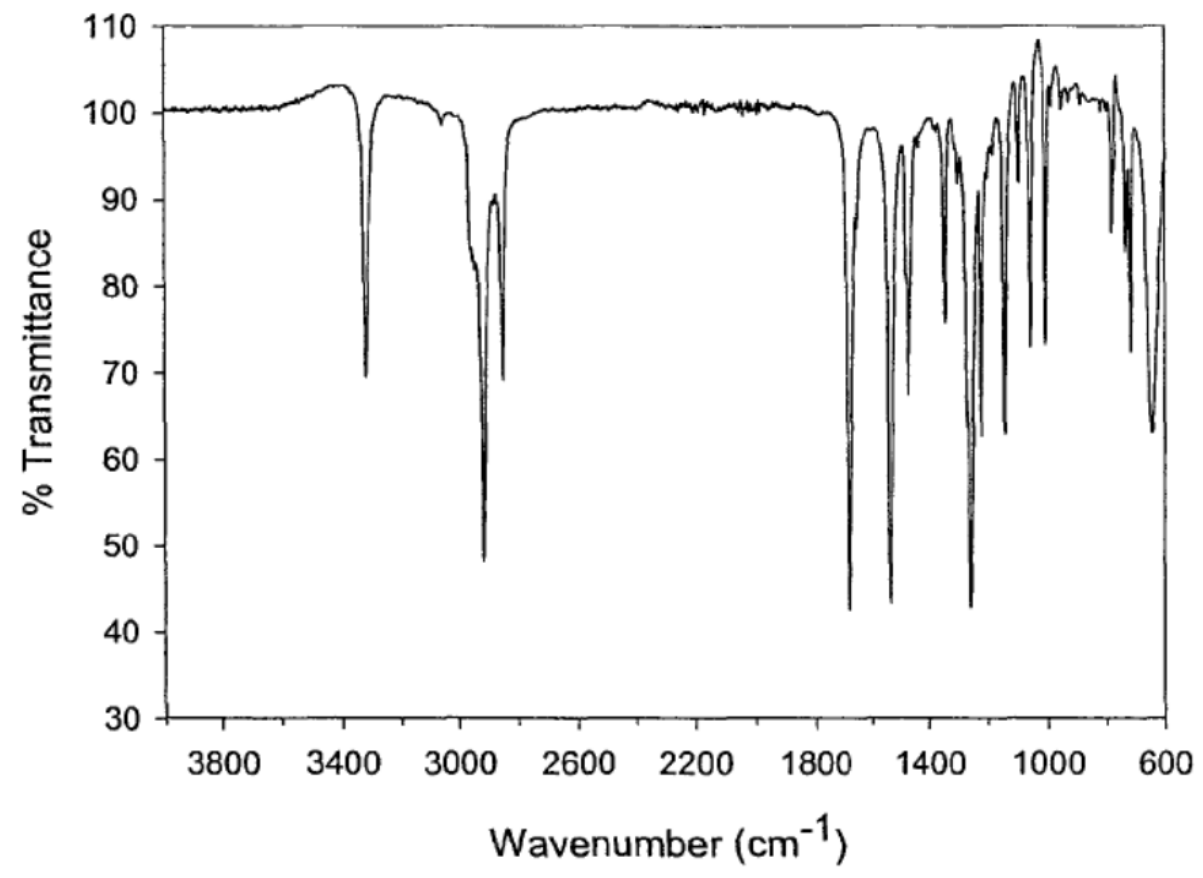

Figure A.9. FTIR spectrum of $\mathrm{C}_{11}$ biscarbamate.

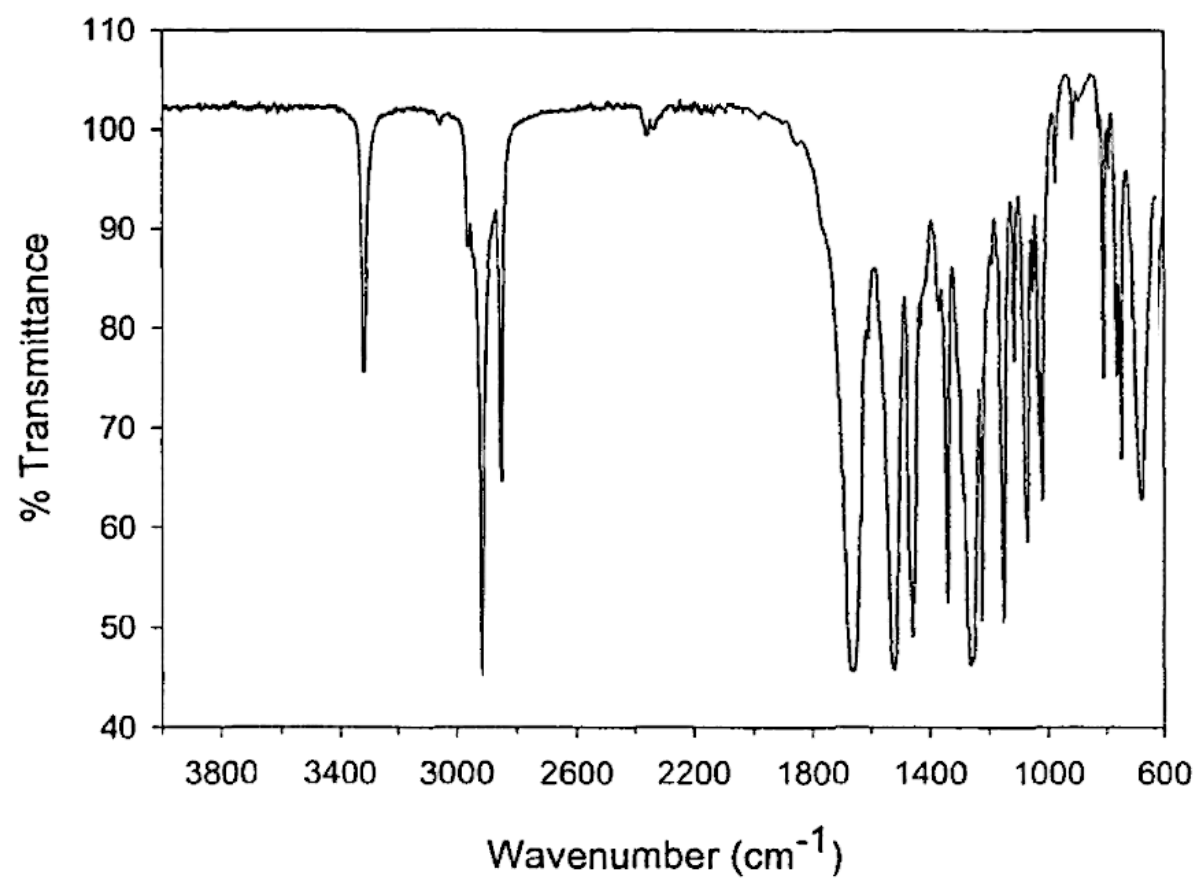

Figure A.10. FTIR spectrum of $\mathrm{C}_{12}$ biscarbamate. 


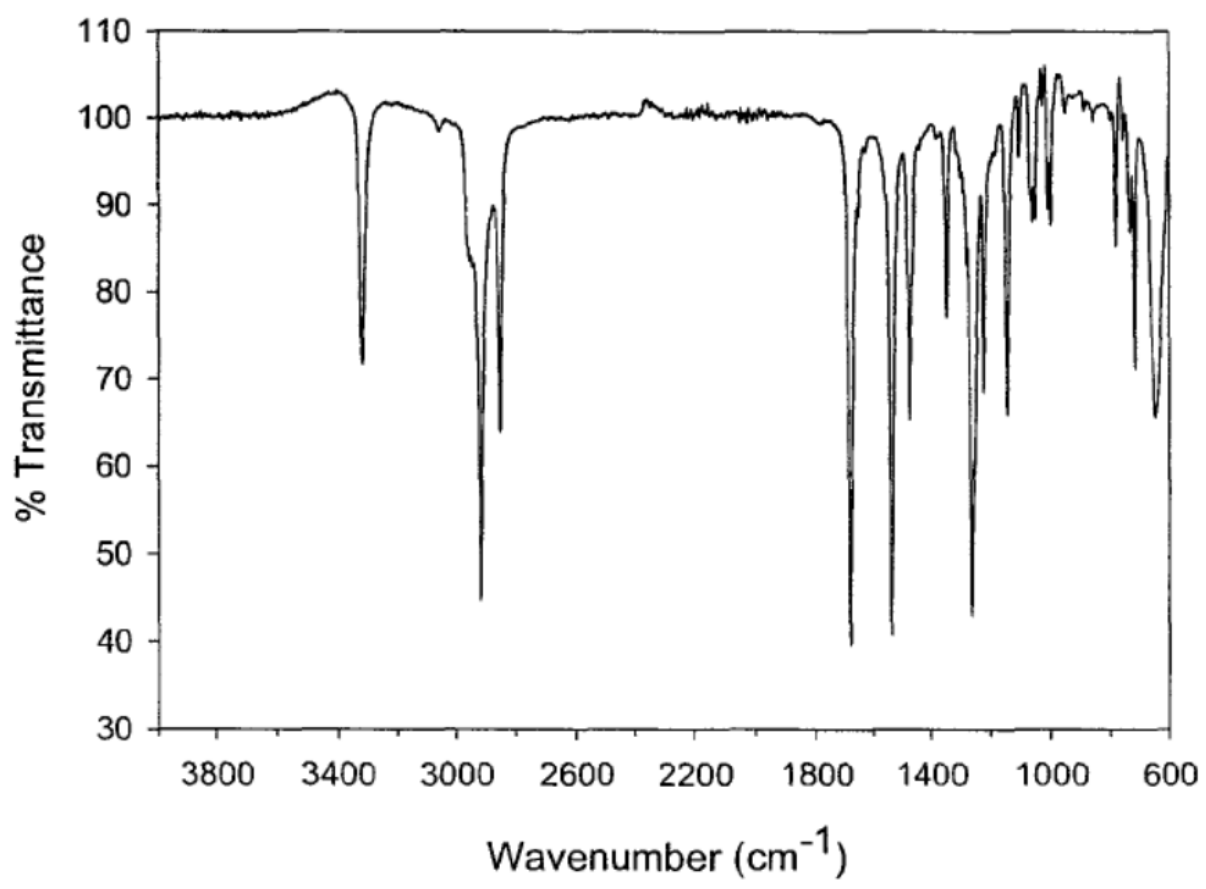

Figure A.11. FTIR spectrum of $\mathrm{C}_{13}$ biscarbamate.

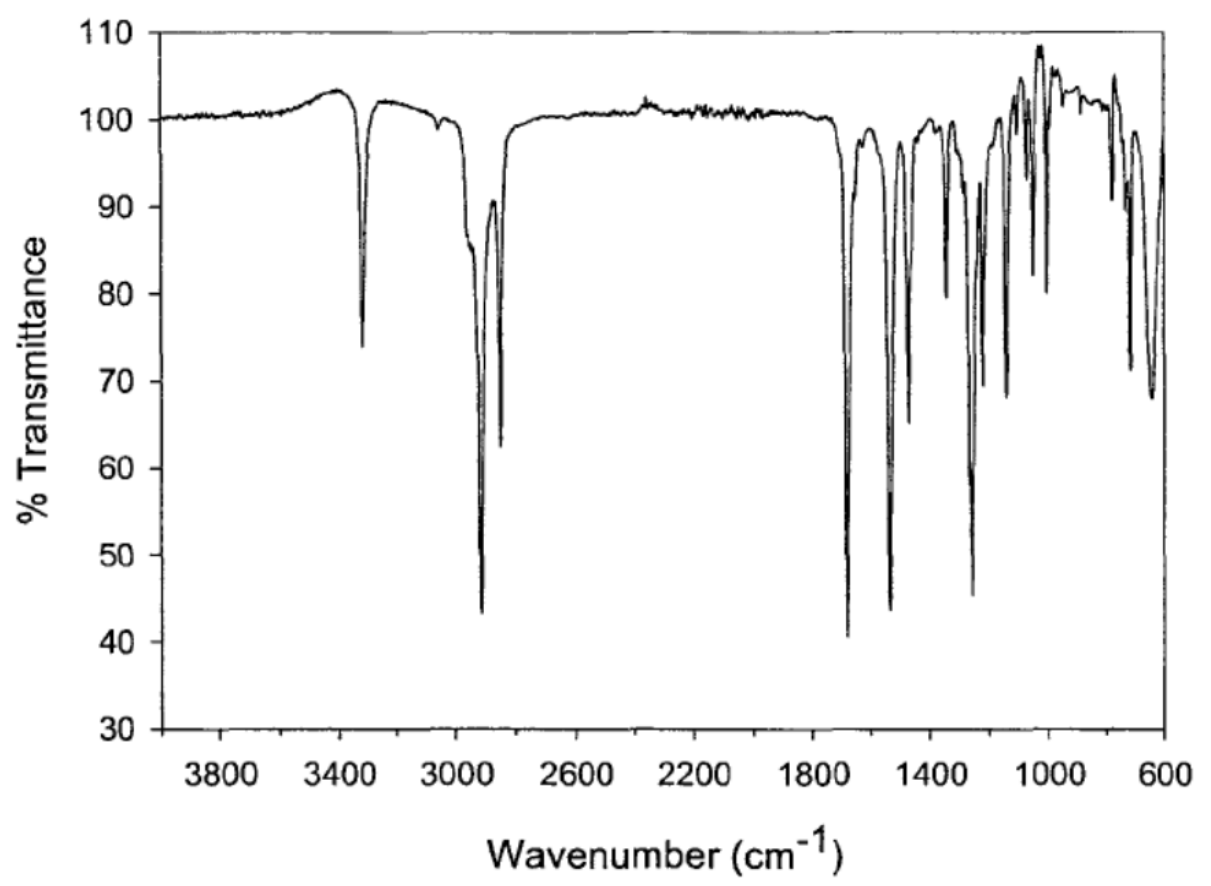

Figure A.12. FTIR spectrum of $\mathrm{C}_{15}$ biscarbamate. 


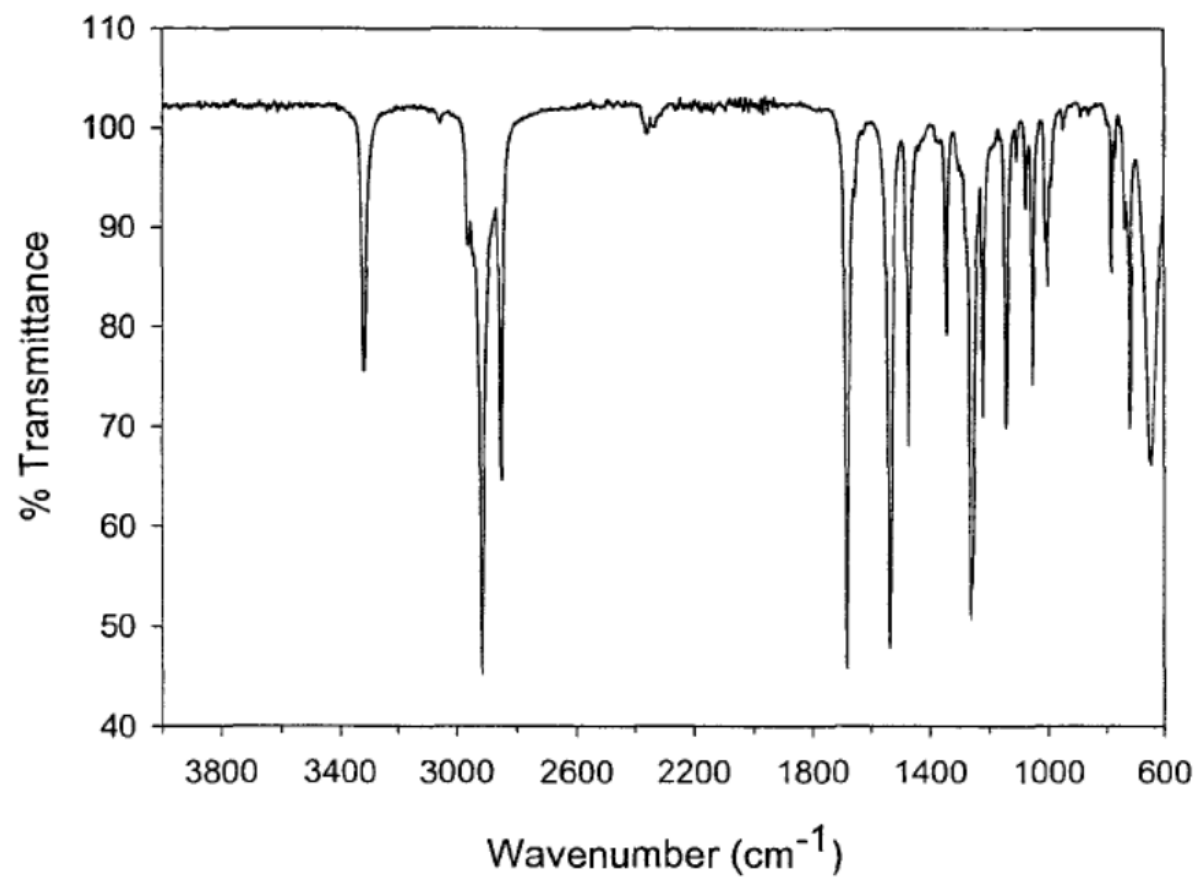

Figure A.13. FTIR spectrum of $\mathrm{C}_{16}$ biscarbamate.

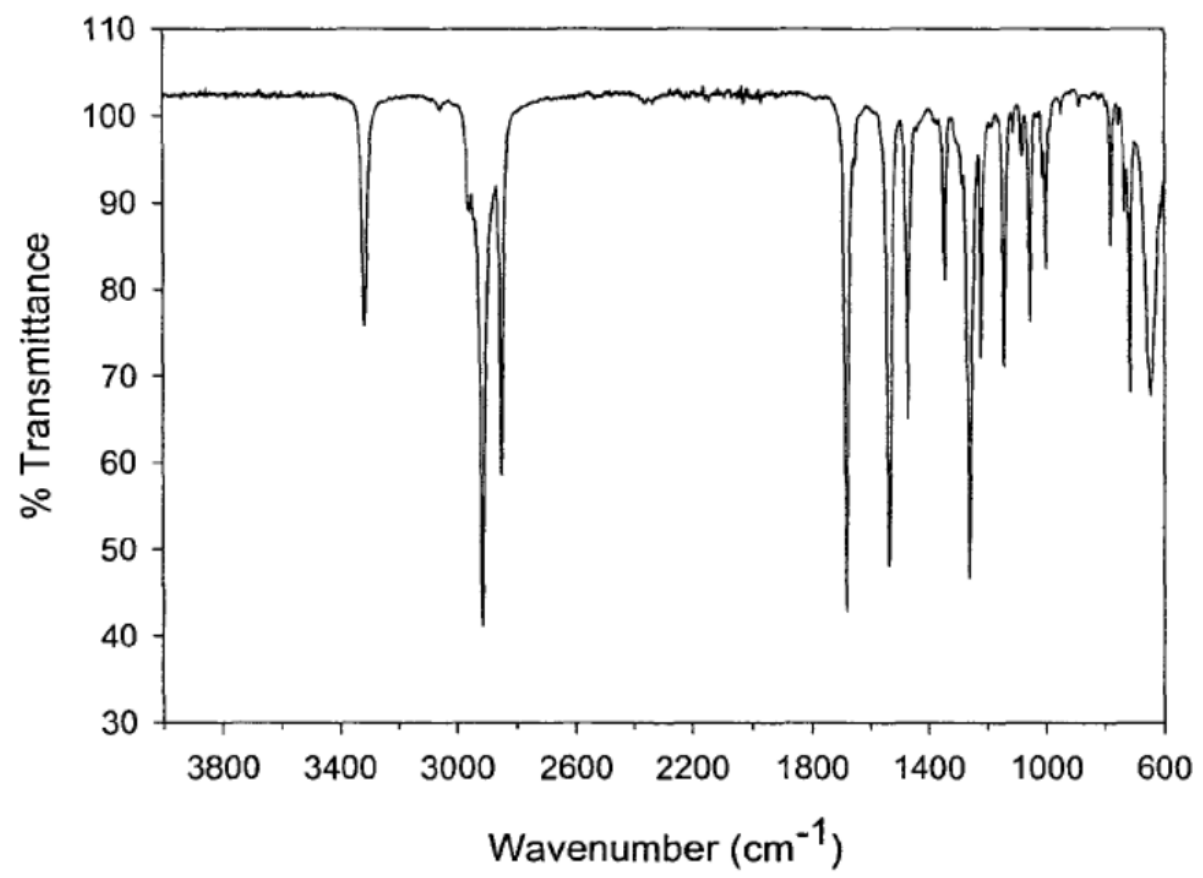

Figure A.14. FTIR spectrum of $\mathrm{C}_{18}$ biscarbamate. 


\section{${ }^{1} \mathrm{H}$ NMR Spectra of the Biscarbamates}




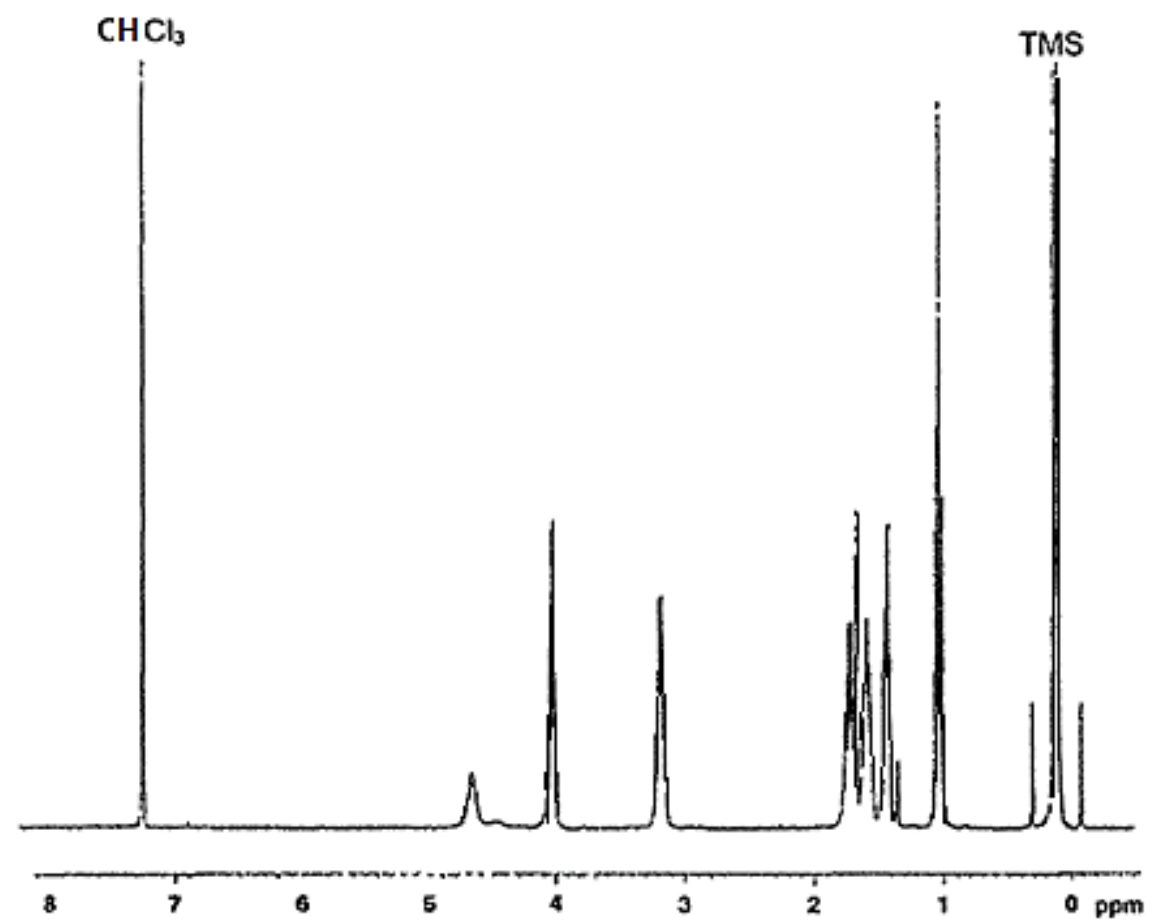

Figure B.1. ${ }^{1} \mathrm{H}$ NMR spectrum of $\mathrm{C}_{3}$ biscarbamate.

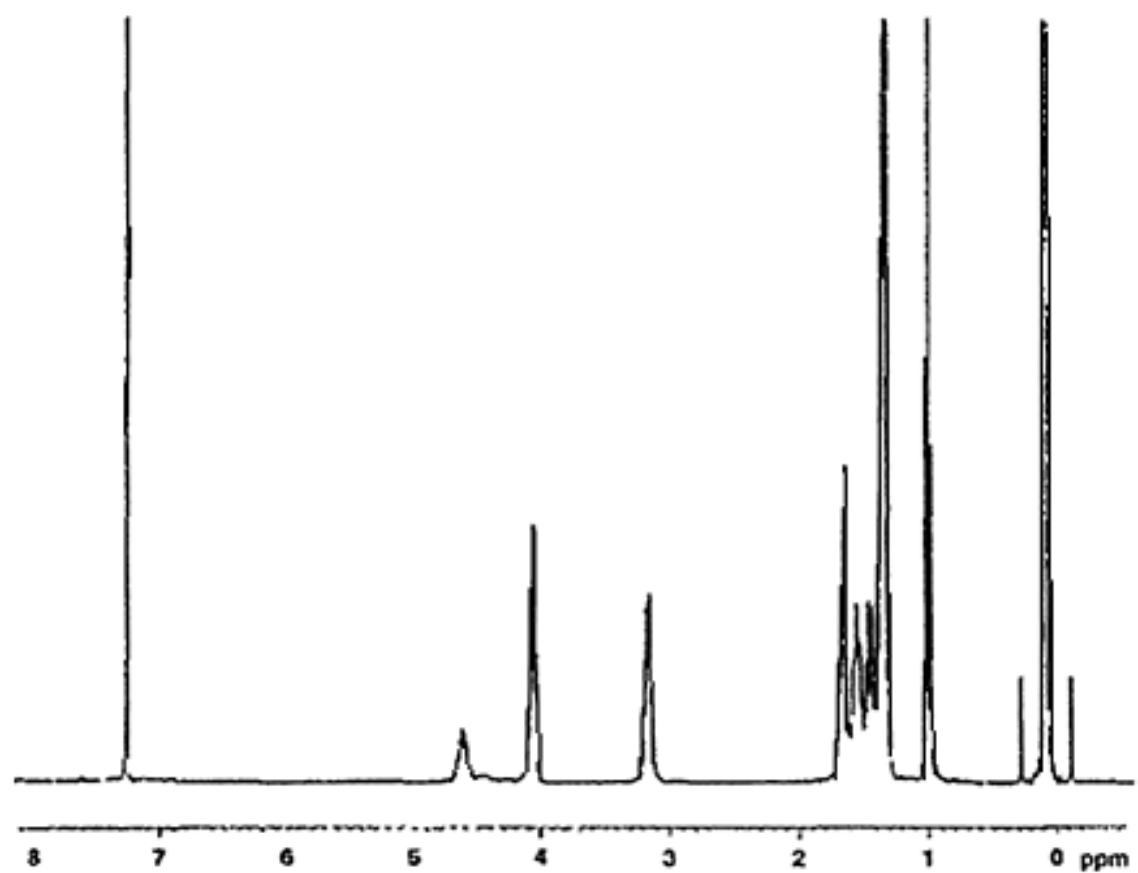

Figure B.2. ${ }^{1} \mathrm{H}$ NMR spectrum of $\mathrm{C}_{4}$ biscarbamate. 


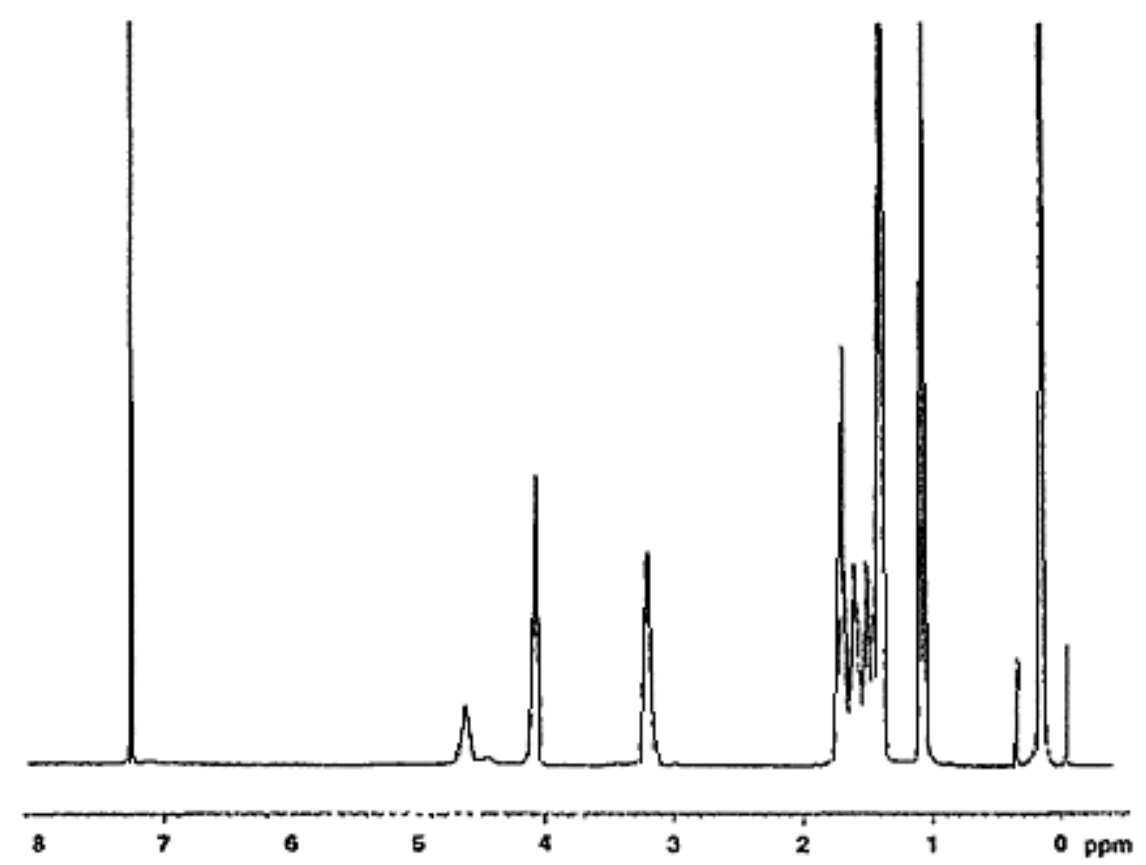

Figure B.3. ${ }^{1} \mathrm{H}$ NMR spectrum of $\mathrm{C}_{5}$ biscarbamate.

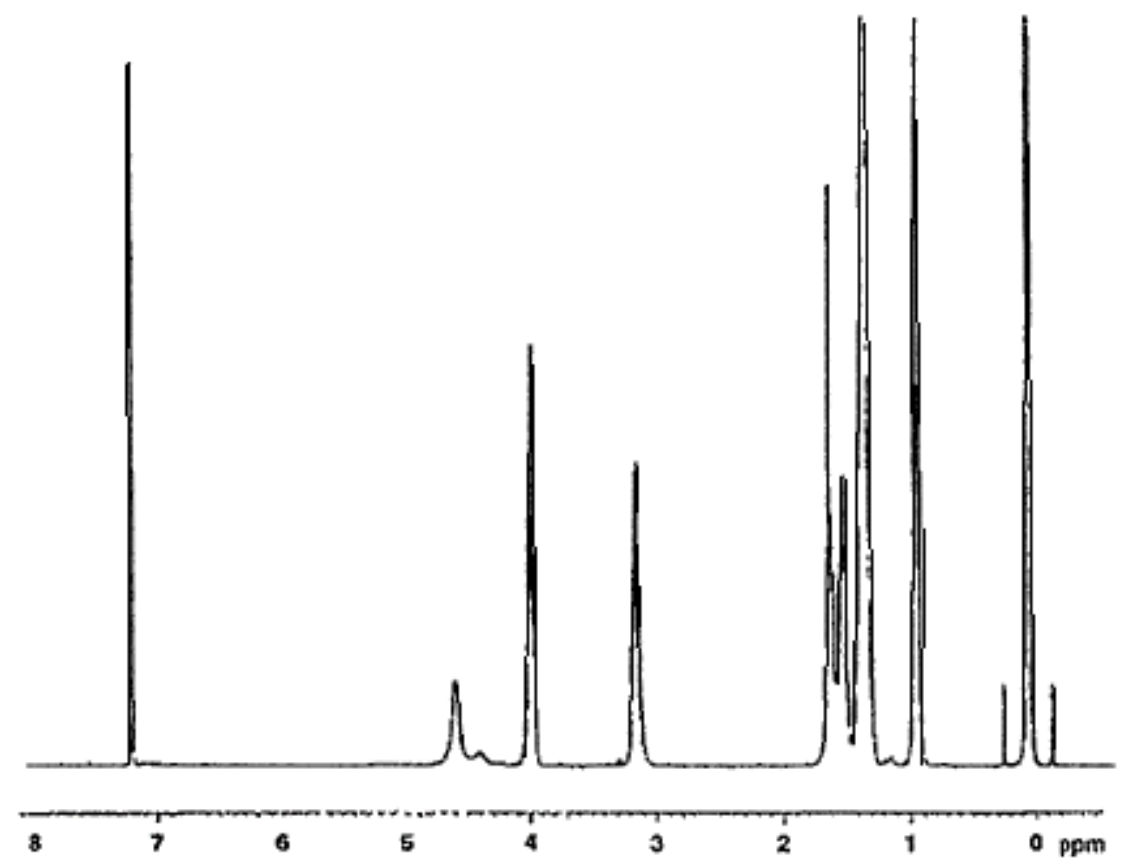

Figure B.4. ${ }^{1} \mathrm{H}$ NMR spectrum of $\mathrm{C}_{6}$ biscarbamate. 


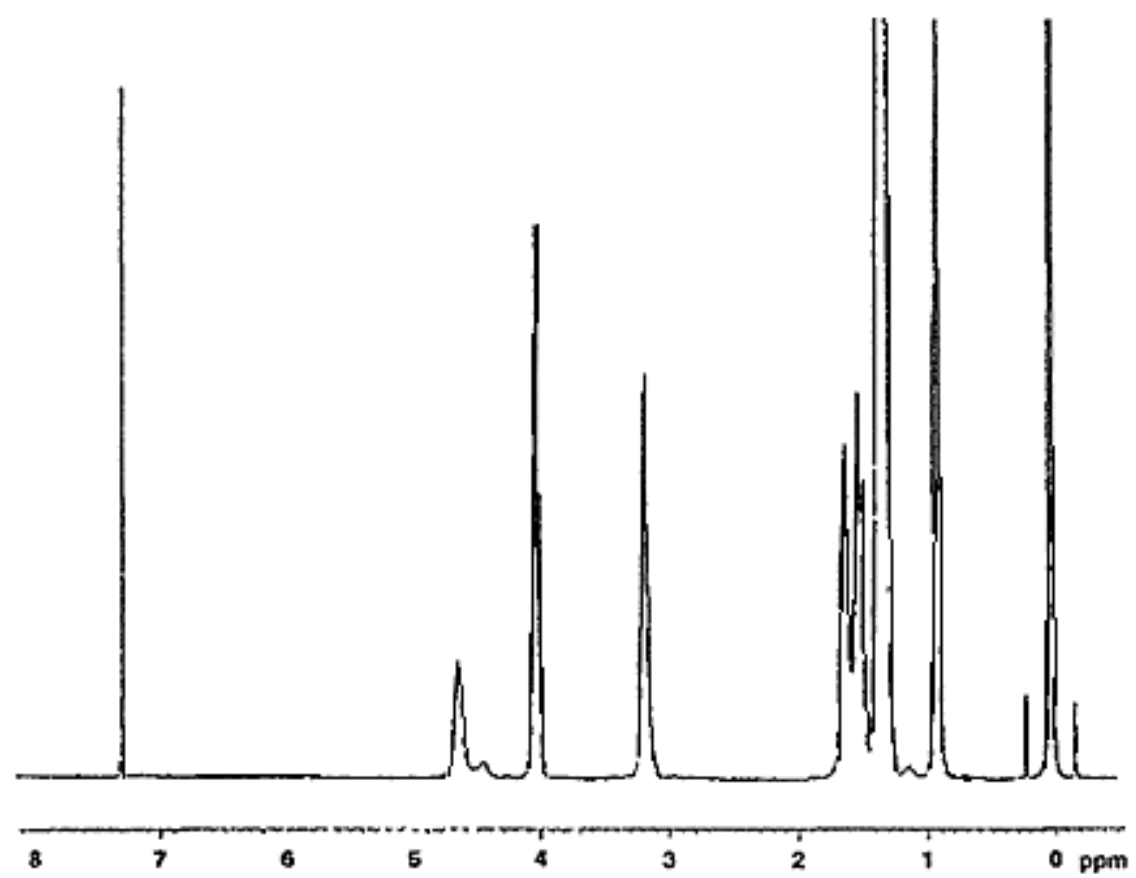

Figure B.5. ${ }^{1} \mathrm{H}$ NMR spectrum of $\mathrm{C}_{7}$ biscarbamate.

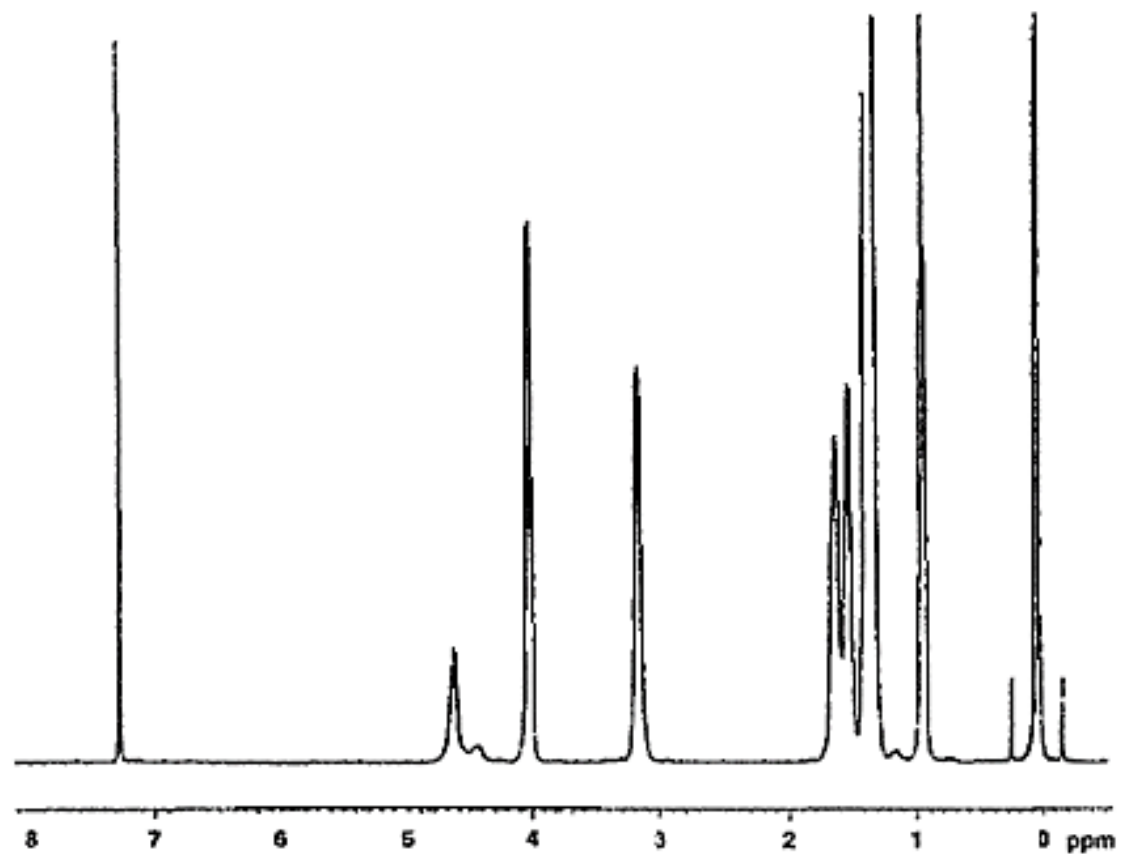

Figure B.6. ${ }^{1} \mathrm{H}$ NMR spectrum of $\mathrm{C}_{8}$ biscarbamate. 


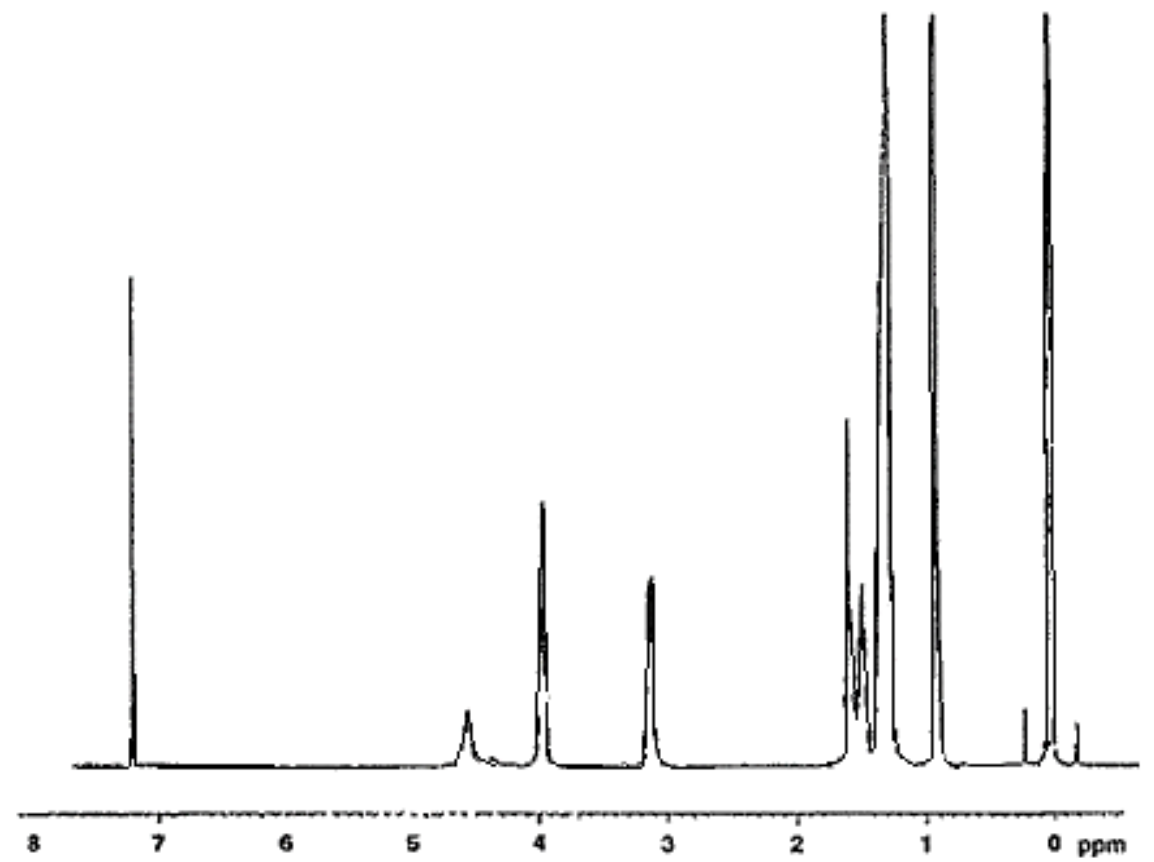

Figure B.7. ${ }^{1} \mathrm{H}$ NMR spectrum of $\mathrm{C}_{9}$ biscarbamate.

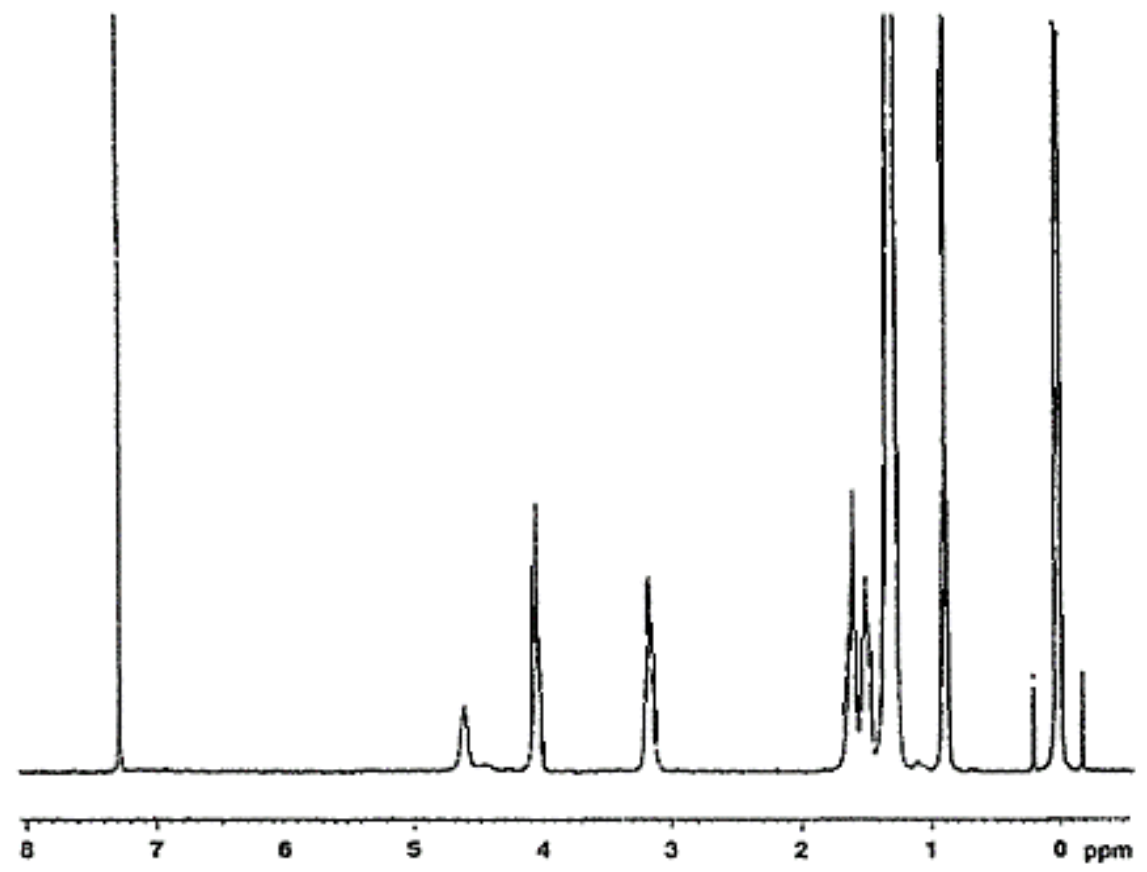

Figure B.8. ${ }^{1} \mathrm{H}$ NMR spectrum of $\mathrm{C}_{10}$ biscarbamate. 


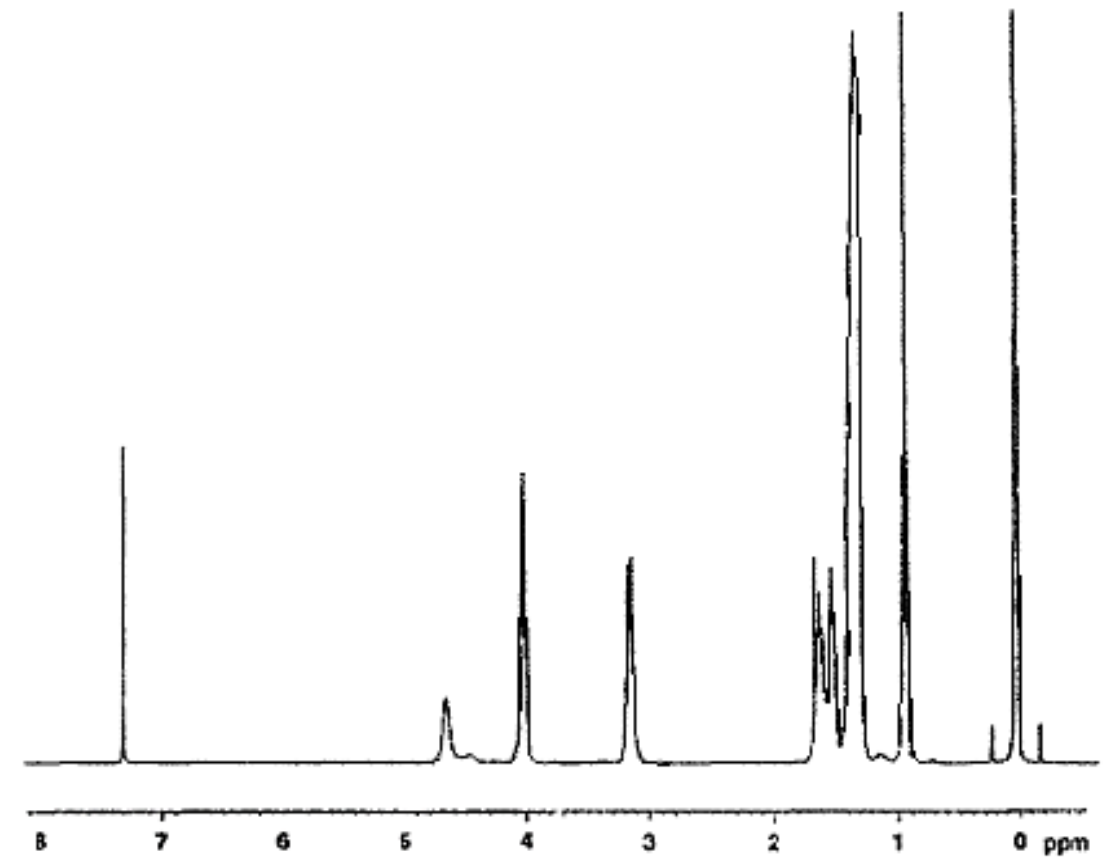

Figure B.9. ${ }^{1} \mathrm{H}$ NMR spectrum of $\mathrm{C}_{11}$ biscarbamate.

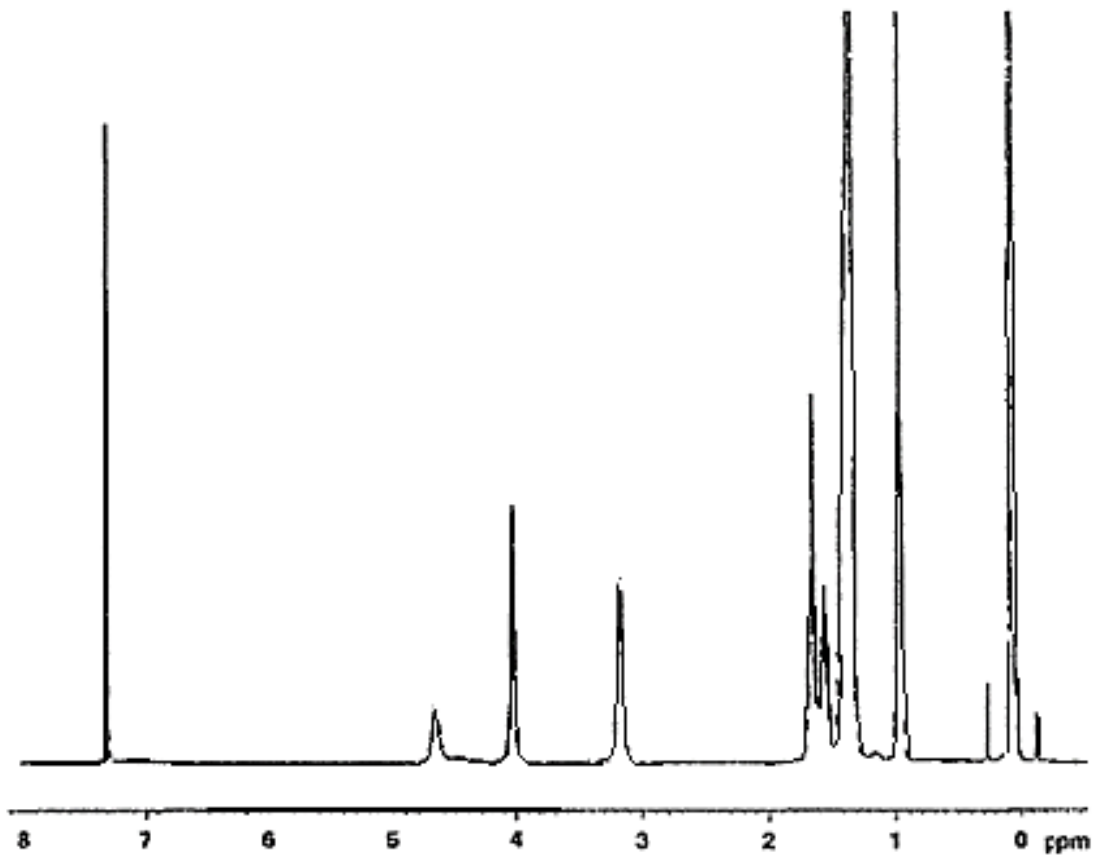

Figure B.10. ${ }^{1} \mathrm{H}$ NMR spectrum of $\mathrm{C}_{12}$ biscarbamate. 


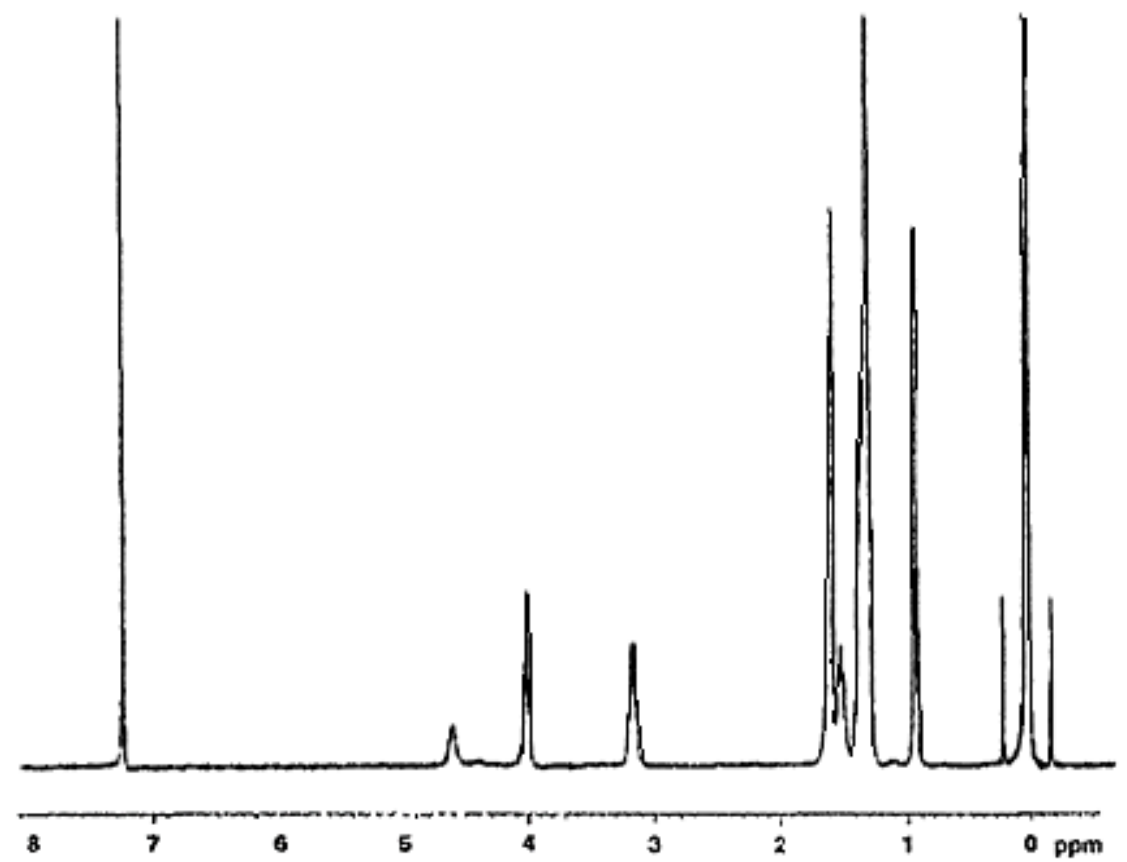

Figure B.11. ${ }^{1} \mathrm{H}$ NMR spectrum of $\mathrm{C}_{13}$ biscarbamate.

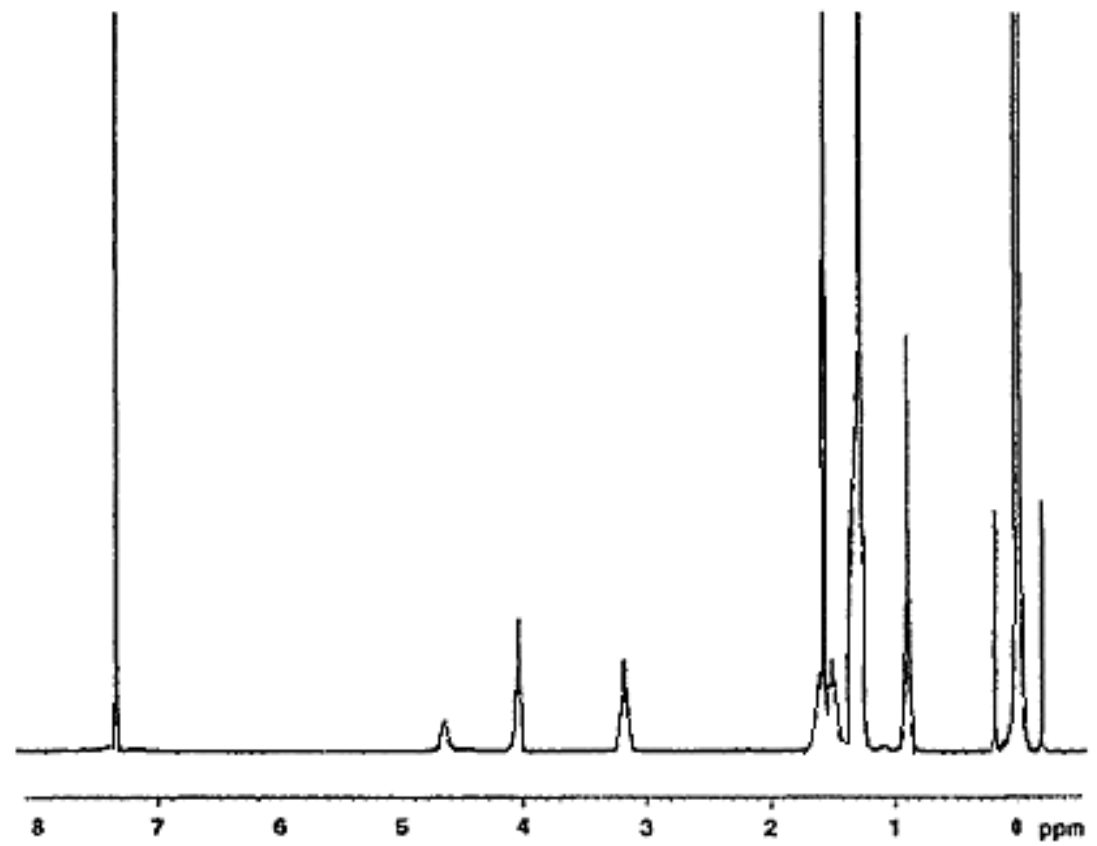

Figure B.12. ${ }^{1} \mathrm{H}$ NMR spectrum of $\mathrm{C}_{15}$ biscarbamate. 


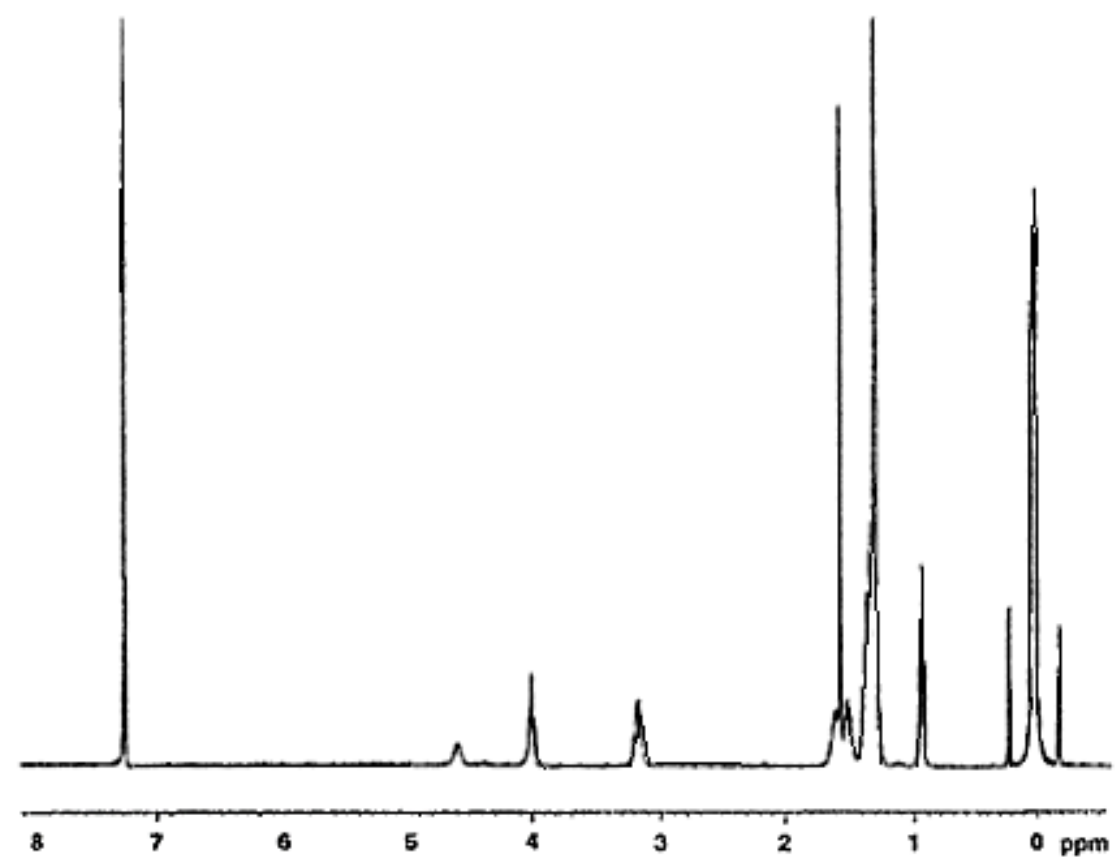

Figure B.13. ${ }^{1} \mathrm{H}$ NMR spectrum of $\mathrm{C}_{16}$ biscarbamate.

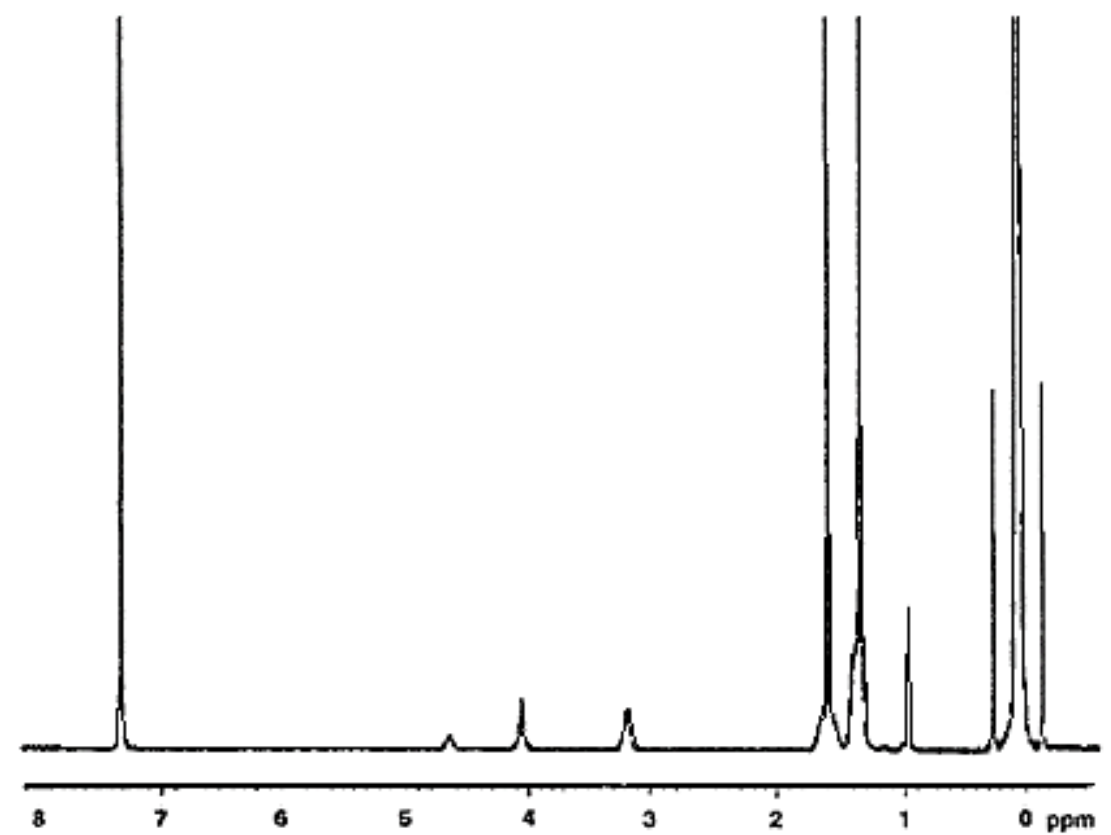

Figure B.14. ${ }^{1} \mathrm{H}$ NMR spectrum of $\mathrm{C}_{18}$ biscarbamate. 